\title{
Studying Prognosis and Treatment in Patients with Neuroendocrine Neoplasia
}

Linde M. van Veenendaal 


\section{Studying Prognosis and Treatment in Patients with Neuroendocrine Neoplasia}



Voor papa en mama 
Studying prognosis and treatment in patients with neuroendocrine neoplasia PhD thesis, Utrecht University, the Netherlands

ISBN: 978-94-6416-047-5

Publication of this thesis was financially supported by Pfizer B.V., Ipsen Farmaceutica B.V., Chipsoft B.V., NET-stichting

Cover design: Wytske van Veenendaal

Book design and lay-out: True color \& Ridderprint B.V. | www.ridderprint.nl

Printing: Ridderprint B.V. | www.ridderprint.nl

(C) Linde M. van Veenendaal, Utrecht, 2020

All right reserved. No part of this thesis may be reproduced or transmitted in any form of by any means without prior written permission from the author. The copyright of the papers that have been published or have been accepted for publication has been transferred to the respective journals 


\title{
Studying Prognosis and Treatment in Patients with Neuroendocrine Neoplasia
}

\author{
De bestudering van prognose en behandeling \\ van patiënten met \\ neuroendocriene tumoren \\ (met een samenvatting in het Nederlands)
}

\begin{abstract}
Proefschrift
ter verkrijging van de graad van doctor aan de Universiteit Utrecht op gezag van de rector magnificus, prof.dr. H.R.B.M. Kummeling, ingevolge het besluit van het college voor promoties in het openbaar te verdedigen op
\end{abstract} donderdag 17 september 2020 des middags te 12.45 uur

door

\section{Linde Michèle van Veenendaal}

geboren op 24 oktober 1985 te Nieuwegein 
Promotoren: $\quad$ Prof. dr. G.D. Valk Prof. dr. M.R. Vriens

Copromotoren: dr. M.E.T. Tesselaar dr. C.M. Korse 


\section{Table of Contents}

Chapter 1 General introduction and thesis outline

\section{Part I Prognosis in Neuroendocrine Neoplasia}

Chapter 2 Survival in patients with neuroendocrine tumours

of the small intestine: nomogram validation and

predictors of survival

(Journal of Clinical Medicine, 2020)

Chapter 3 Molecular prognostic factors in small-intestinal

neuroendocrine tumours

(Endocrine Connections, 2019)

Chapter 4 Merkel cell carcinoma: clinical outcome and prognostic

factors in 351 patients

(Journal of Surgical Oncology, 2018)

Chapter 5 The clinical utility of neuron-specific enolase serum

levels as a biomarker for Merkel cell carcinoma

(Annals of Surgical Oncology, 2020)

\section{Part II Treatment of Patients with Neuroendocrine Neoplasia}

Chapter 6 Clinical benefit of systemic treatment in patients with advanced pancreatic and gastro-intestinal neuroendocrine tumours according to ESMO-MCBS and ASCO framework (Annals of Oncology, 2017) 

Chapter 7 Comparison of transarterial bland embolization and selective internal radiation therapy in patients with metastatic neuroendocrine tumours (Submitted for publication)

Chapter 8 Efficacy of isolated limb perfusion in patients with Merkel cell carcinoma: a multicenter experience (European Journal of Surgical Oncology, 2017)

\section{Part III Summary, General Discussion and Future Perspectives}

Chapter 9 Summary

Chapter 10 General discussion and future perspectives

\section{Appendices}

Dutch summary | Nederlandstalige samenvatting

Contributing authors and affiliations

List of publications

Review committee 


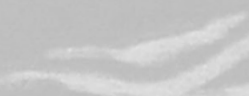

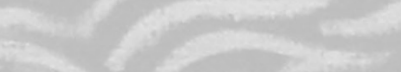

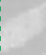

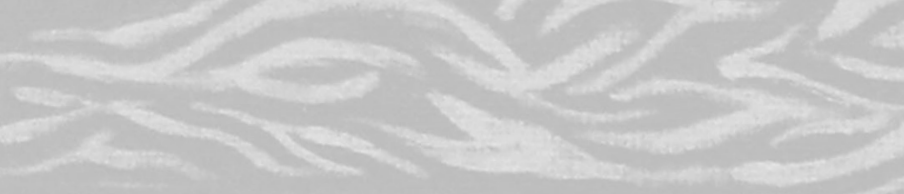




\section{Chapter 1}

General introduction and thesis outline 



\section{General introduction}

Neuroendocrine neoplasia (NEN) represent malignancies that arise from neuroendocrine cells throughout the body. They are often referred to as a homogeneous group of neoplasms, especially when it concerns gastroenteropancreatic neuroendocrine tumours (GEP-NETs). Although they might appear similar histologically, they differ in hormonal production, molecular profile, and clinical aggressiveness based on anatomical location. ${ }^{1-3}$ These differences have important implications for prognosis and treatment. ${ }^{4}$ Unfortunately, until recently their heterogeneity has not been fully recognized and literature often focussed on NEN or GEP-NETs as a whole or in case of Merkel cell carcinoma (MCC) within a group of rare malignancies of the skin, hampering research and the understanding of these malignancies. ${ }^{5-10}$

- History: origin and nomenclature

The history of NEN, which is characterised by complex nomenclature and classifications, contributed to the misconceptions regarding the diversity of these neoplasia. The first neuroendocrine malignancy was described in 1867 by a pathologist Theodor Langhas. ${ }^{11}$ He found a small firm tumour in the ileum, different in appearance from previously described carcinoma in the small bowel. It was in 1907 that Siegfried Obendorfer categorized these type of tumours as 'karzinoid' or carcinoma-like, believing they were benign tumours of the gastrointestinal tract. ${ }^{12}$ In 1938 Friedrich Feyrter developed the concept of a 'diffuse endocrine system', endocrine cells present throughout the body with a preference to the intestinal tract. ${ }^{13}$ All tumours originating from this system were named carcinoids with ileal neuroendocrine tumours referred to as classical carcinoids. ${ }^{14}$

In 1963 carcinoid tumours were classified according to the foregut (respiratory tract, thymus, oesophagus, stomach, duodenum, ovaries and pancreas) midgut (jejunum, ileum, appendix, caecum, ascending colon and Meckel's diverticulum) or hindgut (transversum, descending and sigmoid colon and rectum). ${ }^{15}$

Although carcinoids were considered indolent tumours, some showed a high malignant potential depending on histological diffrentation. ${ }^{16}$ Therefore, the WHO classification (2000) suggested the following categories: 1) well differentiated (neuro)endocrine tumours, 2) well differentiated (neuro)endocrine carcinomas and 3) poorly differentiated (neuro)endocrine carcinomas. ${ }^{17}$ It was generally agreed that the 
term "carcinoid" should no longer be used for NEN, although it is still accepted for NETs originating from the pulmonary system. ${ }^{18,19}$

In 2010, the WHO classification was updated and the general terms neuroendocrine tumour (NET) and neuroendocrine carcinoma (NEC) were introduced based on differentiation. ${ }^{20}$ Further histologic grading was based on the $\mathrm{Ki}-67$ index or mitotic index that should be counted in tumour hot spots. In that grading system NET consisted of a well differentiated NET grade 1 or grade 2, while a NEC represented a poorly differentiated tumour (grade 3 ). In the 2010 WHO grading system, confusion existed regarding the discrepancy between grade and differentiation. ${ }^{21,22}$ Based on this grading system, a morphologically well-differentiated NET with a high Ki-67 level was classified as grade 3 NEC.

The WHO grading system was revised in 2017 for neuroendocrine neoplasia of the pancreas and the above-mentioned issues have been reflected in the changes. ${ }^{23}$ In 2019 the WHO extended this classification for all gastrointestinal NENs, classifying NET as a well differentiated tumour grade 1, 2 or 3 according the Ki-67- or mitotic index and NEC as a poorly differentiated tumour.

- Incidence

Additionally, an significant factor contributing to the lack of attention for their heterogeneity in NEN research is the relatively low incidence, especially taking into account the various types of NEN. ${ }^{24-28}$ For such a highly heterogeneous malignancy large patient populations are necessary to account for the differences, especially in prognostic and therapeutic research. Nevertheless, studies are often based on small series or large population based databases. Although much efforts have been made in these studies, small series remain susceptible for confounding while large population based studies often lack detailed information. Consequently, in recent decades relatively modest progress has been made in understanding and treating NEN.

- Personalized cancer care

Noteworthy, precision medicine has made an entrance in the field of oncology. It aims to provide cancer diagnosis, prognosis and treatment tailored specifically to the individual patient to provide optimal cancer care. ${ }^{29}$ Essential for an adequate personalized treatment is insight on prognosis and treatment with respect to the various tumour and patient characteristics. This emphasizes the need for research focussing on specific types of NEN. In this thesis, the main focus will be upon 
neuroendocrine tumours of the small intestine (SI-NETs) and primary NECs of the skin, also called MCC.

\section{- Small intestine NETs}

Within the group of GEP-NETs, SI-NETs are second most prevalent after rectum NETs and followed by pancreatic NETs. ${ }^{30}$ Moreover, SI-NETs are the most frequent cancer type of the small intestine. ${ }^{31}$ The reported incidence has increased over the last four decades, from 0.2 per 100.000 individuals in 1973 to 1.25 per 100.000 individuals in 2012 and 6.61 per 100.000 individuals in 2011. 2,4 $^{2}$ This progressive rise can mainly be contributed to the more frequent use and improvements of diagnostic modalities and alterations in pathological disease definitions. ${ }^{26}$

Patients with NEN may experience various symptoms from the tumour mass or the output of hormones secreted by the tumour. When clinical symptoms are caused by hormones produced by the neoplasm, it is considered a functional tumour. ${ }^{32}$ In patients with metastatic SI-NETs functional symptoms are often present and referred to as the carcinoid syndrome, which is characterised by diarrhoea, episodic flushing, bronchospasm and sometimes right valvular dysfunction leading to carcinoid heart disease. ${ }^{33}$ Tumours are classified as non-functional if they are clinically (hormonal) silent. Nevertheless, despite the absence of functional symptoms, immunohistochemical staining of hormones are often positive. ${ }^{34}$ Thus, a clear correlation between positive staining and functionality is not always present.

Nowadays, the only potentially curative treatment for patients with a SI-NET consists of surgery. ${ }^{35,36}$ However approximately $40 \%$ of patients have metastases at time of diagnosis, predominantly in the liver. ${ }^{37,38}$ Only a minority of patients (20-30\%) with metastasized SI-NETs is eligible for curative surgery. ${ }^{39}$ In patients with irresectable metastatic disease, resection of the primary tumour could result in a survival benefit. ${ }^{40-42}$ Other non-curative systemic treatment options include the well known somatostatin analogues (SSA). ${ }^{43}$ More recent treatment options, which have shown efficacy in randomized controlled trials are the so called, targeted agents and peptide receptor radionuclide therapy (PRRT) and chemotherapy. ${ }^{44,45}$ Newer options for the treatment of liver metastasis are trans arterial embolization (TAE) and selective internal radiation therapy (SIRT). ${ }^{46}$ Although both treatments are targeted at liver metastases, comparative studies are lacking. 
Even though most patients present with metastatic disease, survival has been shown to be favourable. ${ }^{47-48}$ Several studies have identified prognostic factors in patients with SI-NETs. Advanced dissemination of disease and grading are widely accepted as poor prognostic factors for survival. ${ }^{49-51}$ Most notably, the presence of liver metastases has a strong negative impact on prognosis and causes significant morbidity for patients with a SI-NET. ${ }^{52}$ The 5 -year overall survival for patients with untreated liver metastases is reported to be $24-52 \%$ compared to approximately $75 \%$ for patients with localized resectable disease. ${ }^{53,54}$ Although data is available for cohorts of patients with SI-NET, more insight into the prognosis of individual patients is needed to guide the choices of the patients.

- Merkel cell carcinoma

MCC is a primary NEC of the skin. It was first described as a trabecular carcinoma of the skin by Cyril Toker in $1972 .{ }^{55}$ The tumour cells showed histopathological similarities with normal Merkel cells, which are neuroendocrine cells of the skin and located in the basal layer of the epidermis. Due to their similarities, this trabecular carcinoma was considered to originate from Merkel cells and were referred to as MCC. Although recent insights suggest MCC might originate from other cells, MCC remains the most frequently used and accepted term for this primary cutaneous NEC of the skin. ${ }^{56}$ Like other NEC, MCC are poorly differentiated tumours.

MCC often affects elderly patients and is predominantly located at the head and neck region, followed by the extremities. ${ }^{57,58}$ These sun-exposed locations support the assumption that ultraviolet exposure increases the risk of MCC. Other factors associated with an increased risk are immunosuppression and Merkel cell Polyomavirus. ${ }^{59}$ In rare cases no primary tumour can be found similarly to melanoma, which it is often compared to.

MCC is characterised by an aggressive metastatic spread. At diagnosis, approximately $26 \%$ of the patients have nodal involvement. ${ }^{60}$ Loco-regional recurrence is seen in up to $60 \%$ of the patients, often within two years after diagnosis. ${ }^{61,62}$ Overall survival ranges from 30 to $60 \% .{ }^{63}$ The presence of nodal disease and primary tumour size are identified as predictors for a worse survival. ${ }^{64,65}$ However literature on prognosis in MCC is quite limited.

The rapid spread and poor survival of patients with a MCC justifies an aggressive approach in its treatment, which consists mainly of surgical treatment followed by 
post-operative radiation therapy (RT) for early stage MCC. ${ }^{66}$ Nevertheless, there is no standard treatment for loco regionally advanced disease. Chemotherapy has shown high reponse rates, however seldom durable. ${ }^{67}$

Recent findings in immunology and results from treating patients with immune checkpoint inhibitors have completely changed the treatment landscape and outlook for metastatic MCC patients showing promising results inducing durable partial or complete response rates. ${ }^{67-69}$ The introduction of immunotherapy and subsequently the improved survival of patients with metastatic MCC stresses the need for an accurate biomarker guiding treatment decisions. 



\section{Thesis outline}

The Netherlands Cancer Institute and UMC Utrecht form an European

Neuroendocrine Tumour Society (ENETS) Centre of Excellence specialized in treating patients with NEN. Because of this collaboration patients can benefit from an experienced multidisciplinary team providing them with all the current relevant treatment options. Furthermore, it provides research opportunities due to concentrated care in a relatively large patient population with detailed information on treatment and survival. More understanding of prognostic factors and treatment effects could improve clinical outcome for individual patients with NEN. This thesis aims to analyse prognosis, possible biomarkers and various treatments in patients with a SI-NET and MCC. Unique for this research project is the active collaboration with the Dutch NET and NEC patient Advocacy Group (Stichting NETgroep).

The first part of this thesis focuses on prognostication of patients with a SI-NETs and MCC. Predicting prognosis for an individual patient with a SI-NET is challenging due to their heterogeneous disease course. As a consequence, there remains an unmet need for adequate prognostication to predict disease course and survival for individual patients with SI-NETs. In 2010 Modlin et al developed the first SI-NET nomogram based on clinical and tumour characteristics to estimate disease specific survival on patient level. This nomogram was the first attempt to develop a prognostic tool to asses survival and guide therapy in individual patients with SINETs. In Chapter 2 this nomogram was evaluated within our large institutional cohort $(n=400)$. In addition, prognostic predictors for survival were identified, which could contribute to further development of a prognostic model. In more recent years, basic research in SI-NETs has attempted to unravel the molecular events underlying SI-NET tumorigenesis and identify possible targets for therapy. In Chapter 3 we provide an overview of the current literature regarding prognostic and predictive molecular factors in patients with SI-NETs.

Since literature on MCC is quite limited, clinical outcome and prognostic factors in a large cohort of patients with MCC $(n=351)$ were analysed in Chapter 4. As a prognosticator, serum neuron-specific enolase (NSE) has been validated in earlier studies and is now commonly used as a biomarker in GEP-NETs and several other small cell malignancies. However, the role of NSE in MCC is still unclear. Chapter $\mathbf{5}$ evaluates the role of NSE as a biomarker in a prospectively collected cohort of patients with MCC $(n=84)$ and NSE samples (565 samples). The prognostic value 
and clinical utility of NSE, with a separate analysis in patients receiving immunotherapy, was investigated.

The second part of this thesis concentrates on various therapies available for patients with a SI-NET and MCC. An overview of existing literature about trials comparing systemic antitumor treatment for GEP-NETs is presented and their clinical benefit is interpreted in Chapter 6. Trans arterial embolization (TAE) and selective internal radiation therapy (SIRT) are treatment modalities for patients with liver metastases of a NET. Chapter 7 evaluates the safety and efficacy of TAE and SIRT in patients with (mainly GEP-) NETs with liver-dominant disease. A separate analysis was performed for patients with a SI-NET and pancreatic NET.

In MCC loco regional disease is generally managed with surgery and/or radiotherapy. Yet, no standard treatment for loco regionally advanced disease is established. In patients with loco regionally advanced melanoma, isolated limb perfusion (ILP) of the extremities proved to be safe and effective. Still, its role in patients with MCC remains undetermined. In Chapter 8 we describe our experience with ILP in patients with locally advanced MCC.

Finally, in Chapter 9 our observations are summarised and future perspectives are addressed in Chapter 10. 


\section{References}

1. Klöppel G. Neuroendocrine Neoplasms: Dichotomy, Origin and Classifications. Visc Med. 2017 Oct;33(5):324-330.

2. Kunz PL. Carcinoid and neuroendocrine tumors: building on success. J Clin Oncol 2015; 33:1855-1863.

3. Cives M, Soares HP, Strosberg J. Will clinical heterogeneity of neuroendocrine tumors impact their management in the future? Lessons from recent trials. Curr Opin Oncol. 2016 Jul;28(4):359-66

4. Oronsky B, Ma PC, Morgensztern D, et al. Nothing but NET: a review of neuroendocrine tumors and carcinomas. Neoplasia. 2017;19(12):991-1002.

5. Porceddu SV, Veness MJ, Guminski A. Nonmelanoma Cutaneous Head and Neck Cancer and Merkel Cell Carcinoma: Current Concepts, Advances, and Controversies. Journal of clinical oncology : official journal of the American Society of Clinical Oncology. 2015;33:3338-45.

6. Pape UF, Berndt U, Muller-Nordhorn J, et al. Prognostic factors of long-term outcome in gastroenteropancreatic neuroendocrine tumours. Endocrine-related Cancer. 2008;15(4):1083-97.

7. Dasari A, Shen C, Halperin D, et al. Trends in the Incidence, Prevalence, and Survival Outcomes in Patients With Neuroendocrine Tumors in the United States. JAMA Oncology 2017;3:1335.

8. Garcia-Carbonero R, Capdevila J, Crespo-Herrero G, et al. Incidence, patterns of care and prognostic factors for outcome of gastroenteropancreatic neuroendocrine tumors (GEP-NETs): results from the National Cancer Registry of Spain (RGETNE). Annals of oncology : official journal of the European Society for Medical Oncology / ESMO. 2010;21(9):1794-803.

9. Steinman J, Ariyan C, Rafferty B, et al. Factors associated with response, survival, and limb salvage in patients undergoing isolated limb infusion. Journal of Surgical Oncology. 2014;109(5):405-9.

10. Modlin IM, Lye KD, Kidd M. A 5-decade analysis of 13,715 carcinoid tumors. Cancer. 2003;97(4):934-59.

11. Langhans T. Ueber einen Drüsenpolyp im Ileum. Virchows Arch 38:559-60, 1887.

12. Klöppel G. Oberndorfer and his successors: from carcinoid to neuroendocrine carcinoma. Endocr Pathol. 2007 Fall;18(3):141-4.

13. Feyrter F. Über diffuse endokrine epitheliale Organe. Leipzig: J.A. Barth; 1938.

14. Capella C, Heitz PU, Höfler H, et al. Revised classification of neuroendocrine tumours of the lung, pancreas and gut. Virchows Arch. 1995;425(6):547-560. 
15. Williams ED, Sandler M. The classification of carcinoid tumors. Lancet. 1963;1:238239.

16. Pinchot SN, Holen K, Sippel RS, et al. Carcinoid tumors. Oncologist. 2008 Dec;13(12):1255-69.

17. Klöppel G, Couvelard A, Perren A, et al. Mallorca Consensus Conference participants; European Neuroendocrine Tumor Society. ENETS consensus guidelines for the standards of care in neuroendo-crine tumors: towards a standardized approach to the diagnosis of gastroenteropancreatic neuroen-docrine tumors and their prognostic stratification. Neuroendocrinology. 2009;90(2):162-166.

18. Torre M, Barberis M, Barbieri B, et al. Typical and atypical bronchial carcinoids. Respir Med. 1989;83:305-308.

19. Travis WD. The concept of pulmonary neuroendocrine tumours. In: Travis WD, Brambilla E, Muller-Hermelink HK, Harris CC, eds. Pathology and Genetics of Tumours of the Lung, Pleura, Thymus and Heart. Lyon, France: IARC Press; 2004:19Y20.

20. Klöppel G, Rindi G, Perren A, et al. The ENETS and AJCC/UICC TNM classifications of the neuroendocrine tumors of the gastrointestinal tract and the pancreas: a statement. Virchows Arch. 2010;456(6):595-597.

21. Khan MS, Luong TV, Watkins J, et al. A comparison of Ki-67 and mitotic count as prognostic markers for metastatic pancreatic and midgut neuroendocrine neoplasms. British journal of cancer. 2013;108(9):1838-45.

22. Rindi G, Klimstra DS, Abedi-Ardekani B, et al. A common classification framework for neuroendocrine neoplasms: an International Agency for Research on Cancer (IARC) and World Health Organization (WHO) expert consensus proposal. Mod Pathol. 2018;31(12):1770-1786.

23. Inzani F, Petrone G, Rindi G. The New World Health Organization classification for pancreatic neuroendocrine neoplasia. Endocrinol Metab Clin North Am. 2018;47(3):463-470.

24. Youlden DR, Soyer HP, Youl PH, Fritschi L, Baade PD. Incidence and survival for Merkel cell carcinoma in Queensland, Australia, 1993-2010. JAMA Dermatology. 2014;150(8):864-72.

25. Hodgson NC. Merkel cell carcinoma: changing incidence trends. Journal of Surgical Oncology. 2005;89(1):1-4.

26. Hallet J, Law CH, Cukier M, Saskin R, Liu N, Singh S. Exploring the rising incidence of neuroendocrine tumors: a population-based analysis of epidemiology, metastatic presentation, and outcomes. Cancer. 2015;121(4):589-97. 
27. Tsikitis VL, Wertheim BC, Guerrero MA. Trends of incidence and survival of gastrointestinal neuroendocrine tumors in the United States: a seer analysis. Journal of Cancer. 2012;3:292-302.

28. Yao JC, Hassan M, Phan A, et al. One hundred years after "carcinoid": epidemiology of and prognostic factors for neuroendocrine tumors in 35,825 cases in the United States. Journal of Clinical Oncology .2008;26:3063-72.

29. Bode AM, Dong Z. Precision oncology- the future of personalized cancer medicine? NPJ Precis Oncol. 2017 Mar 20;1(1):2.

30. Fraenkel M, Kim M, Faggiano A, et al. Knowledge NETwork. Incidence of gastroenteropancreatic neuroendocrine tumours: a systematic review of the literature. Endocr Relat Cancer. 2014;21(3):R153-R163.

31. Pan SY, Morrison H. Epidemiology of cancer of the small intestine. World journal of gastrointestinal oncology 2011;3:33-42.

32. Ito $\mathrm{T}$, Lee $\mathrm{L}$, Jensen RT. Carcinoid-syndrome: recent advances, current status and controversies. Curr Opin Endocrinol Diabetes Obes. 2018;25(1):22-35.

33. Grozinsky-Glasberg S, Grossman AB, Gross DJ. Carcinoid Heart Disease: From Pathophysiology to Treatment - 'Something in the Way It Moves'. Neuroendocrinology. 2015;101(4):263-73.

34. Bax ND, Woods HF, Batchelor A, et al. Clinical manifestations of carcinoid disease. World J Surg. 1996 Feb;20(2):142-6.

35. Howe JR, Cardona K, Fraker DL, Kebebew E, Untch BR, Wang YZ, et al. The Surgical Management of Small Bowel Neuroendocrine Tumors: Consensus Guidelines of the North American Neuroendocrine Tumor Society. Pancreas. 2017;46(6):715-31.

36. Larouche V, Akirov A, Alshehri S, et al. Management of Small Bowel Neuroendocrine Tumors. Cancers 2019;11(9):1395.

37. Korse $\mathrm{T}$, Taal $\mathrm{B}$, van Velthuysen $\mathrm{M}$, et al. Incidence and survival of neuroendocrine tumours in the Netherlands according to histological grade: Experience of two decades of cancer registry. European Journal of Cancer 2013;49:1975-83.

38. Fisher AT, Titan AL, Foster DS, et al. Management of Ileal Neuroendocrine Tumors with Liver Metastases. Journal of gastrointestinal surgery : official journal of the Society for Surgery of the Alimentary Tract 2019.

39. Rossi RE, Massironi S, Spampatti MP, et al. Treatment of liver metastases in patients with digestive neuroendocrine tumors. Journal of Gastrointestinal Surgery : official journal of the Society for Surgery of the Alimentary Tract 2012;16:1981-92.

40. Guo J, Zhang Q, Bi X, et al. Systematic review of resecting primary tumor in MNETs patients with unresectable liver metastases. Oncotarget. 2016. 7;8(10):17396-17405. 
41. Bertani E, Falconi M, Grana C, et al. Small intestinal neuroendocrine tumors with liver metastases and resection of the primary: Prognostic factors for decision making. International journal of surgery (London, England). 2015;20:58-64.

42. Almond LM, Hodson J, Ford SJ, et al. Role of palliative resection of the primary tumour in advanced pancreatic and small intestinal neuroendocrine tumours: A systematic review and meta-analysis. European Journal of Surgical Oncology (EJSO). 2017.

43. Stueven AK, Kayser A, Wetz C, Amthauer H, Wree A, Tacke F, et al. Somatostatin Analogues in the Treatment of Neuroendocrine Tumors: Past, Present and Future. International journal of molecular sciences. 2019;20(12).

44. Singh S, Carnaghi C, Buzzoni R, et al. Everolimus in neuroendocrine tumors of the gastrointestinal tract and unknown primary. Neuroendocrinology 2018;106:211-220.

45. Strosberg JR, Wolin EE, Chasen BA, et al. First update on overall survival, progressionfree survival, and health-related time-to-deterioration quality of life from the NETTER1 study: 177Lu-Dotatate vs. high dose octreotide in progressive midgut neuroendocrine tumors. JCO. 2018;36(15 supplemental):4099.

46. de Baere T, Deschamps F, Tselikas L, et al. GEP-NETS update: Interventional radiology: role in the treatment of liver metastases from GEP-NETs. Eur J Endocrinol. 2015;172(4):R151-66.

47. Kennedy A, Bester L, Salem R, et al. Role of hepatic intra-arterial therapies in metastatic neuroendocrine tumours (NET): guidelines from the NET-liver-Metastases Consensus Conference. HPB (2015) 17:29-39

48. Lewis MA, Hobday TJ. Treatment of Neuroendocrine Tumor Liver Metastases. International Journal of Hepatology. 2012;2012:1-12.

49. Landerholm K, Zar N, Andersson RE, et al. Survival and prognostic factors in patients with small bowel carcinoid tumour. The British journal of surgery 2011;98:1617-24.

50. Mocellin S, Nitti D. Gastrointestinal carcinoid: epidemiological and survival evidence from a large population-based study $(n=25531)$. Annals of Oncology 2013;24:3040-4.

51. Kelly S, Aalberg J, Agathis A, et al. Predicting Survival of Small Intestine Neuroendocrine Tumors: Experience From a Major Referral Center. Pancreas 2019;48:514-8.

52. Frilling A, Clift AK. Therapeutic strategies for neuroendocrine liver metastases. Cancer 2015;121:1172-86.

53. Modlin IM, Lye KD, Kidd M. A 5-decade analysis of 13,715 carcinoid tumors. Cancer. 2003;97(4):934-959. 
54. Shah CP, Mramba LK, Bishnoi R,et al. Survival trends of metastatic small intestinal neuroendocrine tumor: a population-based analysis of SEER database. Journal of gastrointestinal oncology 2019;10:869-77.

55. Toker C. Trabecular carcinoma of the skin. Arch Dermatol. 1972 Jan;105(1):107-10.

56. Sunshine JC, Jahchan NS, Sage J, et al. Are there multiple cells of origin of Merkel cell carcinoma? Oncogene. 2018 Mar;37(11):1409-1416.57.

57. Poulsen M. Merkel-cell carcinoma of the skin. Lancet Oncol. 2004;5:593-599.

58. Pectasides D, Pectasides M, Economopoulos T. Merkel cell cancer of the skin. Ann Oncol. 2006;17:1489-1495.

59. Schrama D, Ugurel S, Becker JC. Merkel cell carcinoma: recent insights and new treatment options. Curr Opin Oncol. 2012;24:141-149.

60. Harms KL, Healy MA, Nghiem P, et al. Analysis of prognostic factors from 9387 Merkel cell carcinoma cases forms the basis for the new 8th edition AJCC staging system. Ann Surg Oncol. 2016;23:3564-3571.

61. Poulsen M, Round C, Keller J,et al. Factors influencing relapse-free survival in Merkel cell carcinoma of the lower limb-a review of 60 cases. Int J Radiat Oncol Biol Phys. 2010;76: 393-397.

62. Allen PJ, Bowne WB, Jaques DP, et al. Merkel cell carcinoma: prognosis and treatment of patients from a single institution. J Clin Oncol. 2005;23:2300-2309.

63. Albores-Saavedra J, Batich K, Chable-Montero F, et al. Merkel cell carcinoma demographics, morphology, and survival based on 3870cases: a population based study. J Cutan Pathol. 2010;37:20-27.

64. Iyer JG, Storer BE, Paulson KG, et al. Relationships among primary tumor size, number of involved nodes, and survival for 8044 cases of Merkel cell carcinoma. J Am Acad Dermatol. 2014;70:637-643.

65. Lemos BD, Storer BE, lyer JG, et al. Pathologic nodal evaluation improves prognostic accuracy in Merkel cell carcinoma: analysis of 5823 cases as the basis of the first consensus staging system. JAm Acad Dermatol. 2010;63:751-761.

66. Bhatia S, Storer BE, lyer JG, et al. Adjuvant radiation therapy and chemotherapy in Merkel cell carcinoma: survival analyses of 6908cases from the national cancer data base. J Natl Cancer Inst.2016;108.67. checkpoint inhibitors have completely changed the treatment landscape and outlook for metastatic MCC patients showing promising results inducing durable partial or complete response rates

67. Kaufman HL, Russell J, Hamid O, et al. Avelumab in patients with chemotherapyrefractory metastatic Merkel cell carcinoma: a multi-centre, single-group, open-label, phase 2 trial. Lancet Oncol.2016;17:1374-1385. 
Chapter 1

68. Nghiem PT, Bhatia S, Lipson EJ et al. PD-1 Blockade with Pembrolizumab in Advanced Merkel-Cell Carcinoma. New England Journal of Medicine 2016;374:2542-2552.

69. Kaufman HL, Russell JS, Hamid O et al. Updated efficacy of avelumab in patients with previously treated metastatic Merkel cell carcinoma after $>/=1$ year of follow-up: JAVELIN Merkel 200, a phase 2 clinical trial. J Immunother Cancer 2018;6:7. 



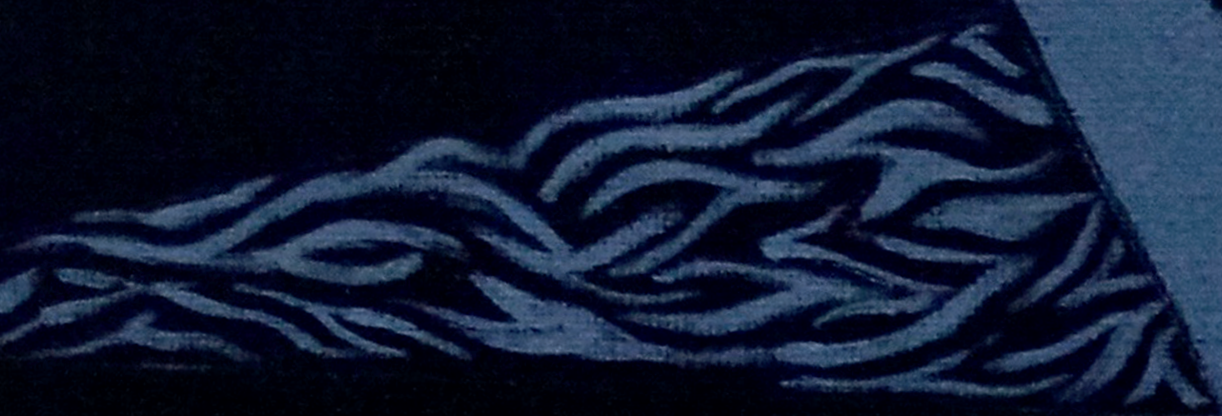




\section{Part I}

Prognosis in Neuroendocrine Neoplasia

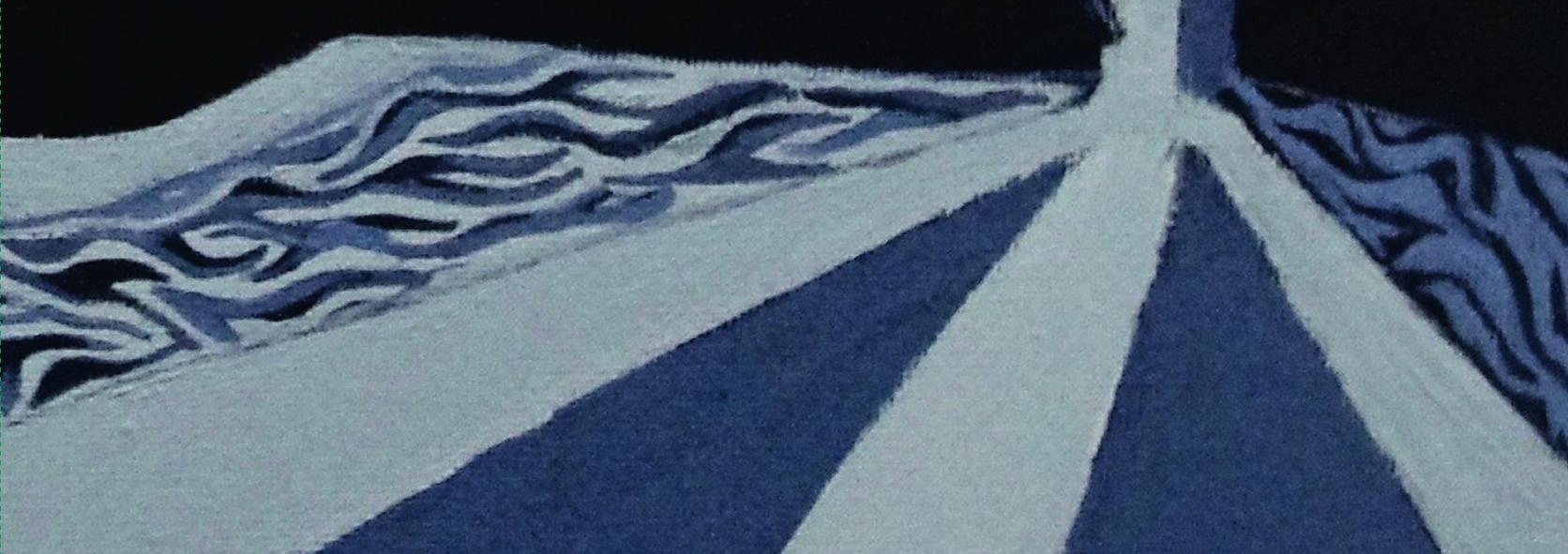




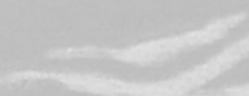

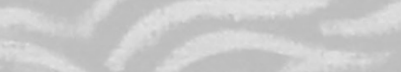

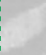

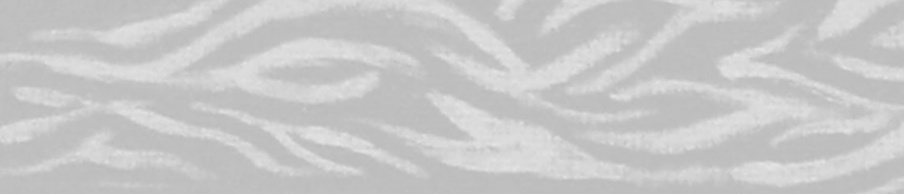




\section{Chapter 2}

Survival in patients with neuroendocrine tumours of the small intestine: nomogram validation and predictors of survival

L.M. van Veenendaal*, S. Levy*, E.C.H. Breekveldt, C.M. Korse, W.H.M. Verbeek, M.R. Vriens, K. Kuhlmann, J.G. van den Berg, G.D. Valk, M.E.T. Tesselaar, * both authors contributed equally

Journal of Clinical Medicine, 2020 


\section{Abstract}

\section{Background}

Neuroendocrine tumours of the small intestine (SI-NETs) are rare with a heterogeneous disease course. There is an unmet need for prognostication to predict disease course and to aid treatment strategies. A previously developed nomogram aims to predict disease specific survival (DSS) in patients with a SI-NET.

\section{Aim}

To validate the nomogram and identify predictors of survival to further improve prognostication.

\section{Patients and Methods}

Four hundred consecutive patients with a grade 1 or 2 SI-NET were included between January 2000 and June 2016. Predicted 5- and 10-year survival was compared to actual DSS. Independent predictors for actual DSS were identified in multivariable analysis.

\section{Results}

The nomogram was able to differentiate between risk groups $(\mathrm{P}<0.001)$. In low-, medium- and high-risk groups 5-year nomogram DSS vs. actual DSS was 0.86 vs. $0.82(\mathrm{P}<0.001)$, 0.52 vs. $0.71(\mathrm{P}<0.001)$ and 0.26 vs. $0.53(\mathrm{P}<0.001)$, respectively. Ten-year nomogram DSS vs. actual DSS was 0.68 vs. $0.69(\mathrm{P}<0.001), 0.40$ vs. 0.50 $(\mathrm{P}<0.001)$ and 0.20 vs. $0.35(\mathrm{P}<0.001)$, respectively. Age, WHO performance score of $2, \mathrm{Ki}-67$ index $\geq 10$, unknown primary tumour, $\mathrm{CgA}>6 \mathrm{x}$ ULN and elevated liver tests were identified as independent predictors for a worse DSS.

\section{Conclusion}

The nomogram was able to differentiate but generally underestimated DSS for patients with a SI-NET. Improvement of prognostication is necessary to adequately estimate survival 


\section{Introduction}

Neuroendocrine tumours (NETs) represent a heterogeneous group of rare tumours, most commonly presented in the gastrointestinal - and bronchopulmonary tract. ${ }^{1-3}$ The incidence of NETs is increasing, with a reported incidence of 6.61 per 100.000 individuals in 2011. ${ }^{1,4}$ NETs of the small intestine (SI-NETs) are the second most common NETs, after pulmonary NETs, and the most frequent malignancy of the small intestine..$^{4-7}$ Up to $73 \%$ of patients have metastases at time of diagnosis, predominantly in the liver. ${ }^{1,2,8,9}$ Currently, the only potentially curative treatment for patients with SI-NETs consists of surgery. ${ }^{10,11}$ Unfortunately, only a minority of patients (20-30\%) with metastasised NETs are eligible for curative surgery. ${ }^{10,12,13}$ In the palliative setting salvage surgery, surgery of the primary tumour, liver-directed therapies, somatostatin receptor analogues (SSAs) and peptide receptor radionuclide therapy (PRRT) are available. ${ }^{11,14,15}$ Even though most patients present with metastatic disease, survival has been shown to be favourable, with a 5-year survival rate of $75 \%{ }^{16-18}$

Still, predicting prognosis for an individual patient with a SI-NET remains challenging due to their heterogeneous disease course. ${ }^{19}$ Several studies have identified prognostic factors mainly based on clinical and tumour characteristics. Nevertheless, their role in daily clinical practice remained limited. ${ }^{20-22}$ Additionally in recent years, the genomic landscape of SI-NETs has been under increasing investigation and identified several molecular prognostic factors. ${ }^{23,24}$ However, at present these factors have not yet been adopted into clinical practice. Additionally, the scientific focus has undergone a shift towards the development of 'liquid biopsies': blood-based biomarkers that can be used in clinical practice to predict disease presence or prognosis. These may constitute of circulating tumour cells (CTC), miRNA or circulating tumour transcripts. In several malignancies, liquid biopsies were able to predict prognosis. ${ }^{25-28}$ In neuroendocrine tumours, both CTC and circulating tumour transcripts, showed promising results for monitoring disease. ${ }^{29-31}$ Yet often in these studies the value of clinical and tumour characteristics is underappreciated. Despite the identification of several prognostic factors and biomarkers, currently there remains an unmet need for adequate prognostication to predict disease course and survival for individual patients with SI-NETs.

In 2010 Modlin et al. developed the first SI-NET nomogram based on clinical and tumour characteristics, to estimate an individual 5- and 10-year disease specific 
survival (DSS). ${ }^{20}$ Two studies have aimed to validate this nomogram for clinical use in daily practice. One study included 121 patients who underwent surgery with curative intent for a SI-NET, another validated the nomogram in 70 patients with a SINET. ${ }^{18,31}$ To date, large validation studies have not been performed to assess the validity of the previously established nomogram and therefore the value of clinical patient and tumour characteristics, in a real-world cohort of patients with a SI-NET with various stages of disease and treatment modalities.

We aim to assess whether prognostication based on this nomogram and the constituting clinical and tumour characteristics is suitable, especially considering the shifting focus to new emerging biomarkers. Therefore, in this study we evaluate the prognostic ability of the nomogram in a large patient cohort treated in an European Neuroendocrine Tumour Society Centre of Excellence (ENETS CoE). In addition, prognostic predictors for survival were identified, which could contribute to further development of a prognostic model.

\section{Patients and Methods}

All patients with a well-differentiated, grade I or grade II SI-NET referred to the Netherlands Cancer Institute ( $\mathrm{NCl}$ ) and University Medical Centre Utrecht (UMCU), an ENETS CoE, from January 2000-June 2016 were included for retrospective analyses. Primarily, diagnosis was histopathology confirmed. When histopathological examination was not sufficient for a definitive diagnosis or in case of an unknown primary tumour, the consensus of a multidisciplinary expert panel was used to establish definitive diagnosis and assign the primary tumour type. Consensus was reached with the use of various parameters such as elevated serum biomarkers: Chromogranin $\mathrm{A}(\mathrm{CgA})$, serotonin in thrombocytes or urinary 5-hydroxyindoleacetic acid (5-HIAA), typical desmoplastic fibrotic reaction in a mesenterial mass on imaging or functional symptoms referred to as the carcinoid syndrome (CS) or the presence of carcinoid heart disease (CHD). CS was considered flushing, diarrhoea and/or wheezing. CHD was confirmed with echocardiography. Patients were excluded if histopathological revision during the study period re-classified the tumour into a grade III NET or NEC. All relevant baseline and follow up characteristics were extracted from the longitudinal institutional neuroendocrine neoplasia database, which includes all patients treated in the centre. Since our centre functions as a tertiary referral centre, date of referral and consequently disease and 
clinical characteristics at time of referral were considered baseline for referred patients $>3$ months after diagnosis. Urinary 5-HIAA and serotonin in thrombocytes levels > upper limit of normal (ULN) were combined into one variable: 'elevated serotonin', since urinary 5-HIAA was replaced by the latter in clinical follow up. Follow-up, vital status and cause of death were recorded. The study was conducted in agreement with the $\mathrm{NCI} / \mathrm{UMCU}$ ethical guidelines and all patients gave consent for the use of their medical data as per institutional protocol.

- Handling of missing variables

Missing values were predicted using multiple imputation. Variables that were assumed to be missing not at random were excluded from multiple imputation (tumour size, Ki-67, tumour grade, World Health Organisation (WHO) performance score and ethnicity). To establish patterns of missing values in the remaining variables Little's missing completely at random (MCAR) was performed. CgA, serotonin and liver tests (including both liver function tests as liver enzymes) were found to be MCAR and were imputed using the fully conditional specification method. For imputation of continuous variables a linear regression was used and for dichotomous variables logistic regression was used. The minimum amount of imputations was determined by the maximum percentage of missing data in the variables.

- Nomogram and prognostic indicators

For all patients individual predicted survival according to the nomogram was calculated. Nomogram survival reflects the predicted 5-year or 10-year DSS. For variables in which missing values were not imputed, best possible and worst possible scenario was created: missing values were assigned no points (scenario 1) or highest possible points (scenario 2), respectively. Hereafter, patients were divided in three equal strata: low-, medium- and high-risk stratum, according to their predicted survival probability. Actual DSS for these strata was calculated using Kaplan-Meier curves and was compared to the nomogram survival (for both scenario 1 and 2) using paired signed rank test. DSS for the three risk strata were compared using the logrank test.

Additionally, the predictive value of the nomogram was evaluated in three patient categories. These categories were assumed to differ in a-priori survival probability: group 1 who underwent surgery with curative intent, group 2 who underwent surgery 
in a palliative setting, such as resection of primary tumour in metastatic setting or debulking surgery, and the final group (3) consists of patients who were not eligible for surgical treatment. For these subgroups, nomogram survival was compared to actual DSS as well. In our institutional patient cohort (both the original dataset as well as in the imputed dataset) a separate analysis to identify independent prognostic indicators for actual DSS was performed.

\section{- Statistics}

Variables were analysed using descriptive statistics: median with interquartile range (IQR) for continuous variables, frequency and percentage for categorical variables, respectively. DSS was calculated from date of diagnosis or date of referral for patients referred $>3$ months after diagnosis. Patients alive before reaching one of the endpoints and patients who died of other causes were censored at their last time of follow-up or death, respectively. DSS and possible prognostic indicators were analysed using Kaplan Meier curves, the log rank test and Cox's proportional hazards regression. Variables with a $\mathrm{P}$-value $<0.2$ were included in multivariable analysis. Variable selection for multivariable analysis was performed using backward stepwise selection retaining variables with a P-value $<0.05$. To avoid collinearity, the absence/presence of CS and CHD were combined in one variable. The same was done for tumour grade and $\mathrm{Ki}-67$, combining these variables in grade I, grade II and $<5 \% \mathrm{Ki}-67$, grade II and $\mathrm{Ki}-67 \geq 5 \%$ but $<10 \%$ and grade $\mathrm{II} \geq 10 \%$. Statistical analyses were performed using IBM SPSS Statistics software, version 25.0.

\section{Results}

A total of 400 patients were included. Patient characteristics at baseline and nomogram variables can be found in Table 1. In the cohort 192 patients (48\%) were male and 208 patients (52\%) were female. Median age was 63 years (IQR 55-71). A total of 244 patients $(61 \%)$ were referred within 3 months of diagnosis. Median time to referral for the remaining patients was 18 months (IQR 7-57 months). Patients had a WHO performance status of 0, 1, and 2 in 161 (40\%), $123(32) \%$ and 34 (9\%) patients, respectively. The ethnicity records were missing in 130 patients (33\%). However, within the remaining 270 patients, the majority ( $\mathrm{n}=253,93 \%)$ was Caucasian, seven patients (3\%) were Black and ten patients $(4 \%)$ had another 
Table 1: Baseline characteristics

\begin{tabular}{|c|c|c|}
\hline Characteristic & $\begin{array}{r}\text { n(\%) or median } \\
\text { (IQR, range) }\end{array}$ & $\begin{array}{r}\text { imputed } \\
\mathbf{n}(\%)\end{array}$ \\
\hline Male & $192(48 \%)$ & \\
\hline Female & $208(52 \%)$ & \\
\hline Age at diagnosis (years) & $63(55-71)$ & \\
\hline Time to referral (months) & $18(7-57)$ & \\
\hline \multicolumn{3}{|l|}{ WHO PS: } \\
\hline $\mathbf{0}$ & $161(40.3 \%)$ & \\
\hline 1 & $123(32.3 \%)$ & \\
\hline 2 & $34(8.6 \%)$ & \\
\hline Missing & $76(19 \%)$ & \\
\hline \multicolumn{3}{|l|}{ Ethnicity:* } \\
\hline Caucasian & $253(63.2 \%)$ & \\
\hline Black & $7(1.8 \%)$ & \\
\hline Other & $10(2.5 \%)$ & \\
\hline Missing & $130(32.5 \%)$ & \\
\hline \multicolumn{3}{|l|}{ Primary tumour: } \\
\hline SI & $304(76.0 \%)$ & \\
\hline Unknown primary & $96(24.0 \%)$ & \\
\hline Carcinoid syndrome* & $267(66.8 \%)$ & \\
\hline CHD* & $24(6.0 \%)$ & \\
\hline Distant metastases at referral & $305(76.3 \%)$ & \\
\hline Liver metastases at referral* & $236(59.0 \%)$ & \\
\hline \multicolumn{3}{|l|}{ Tumour grade:* } \\
\hline 1 & $265(66.3 \%)$ & \\
\hline 2 & $94(23.5 \%)$ & \\
\hline Unknown & $41(10.3 \%)$ & \\
\hline \multicolumn{3}{|l|}{ Tumour size:* } \\
\hline$<2$ & $44(11.0 \%)$ & \\
\hline $2-2.5$ & $22(5.5 \%)$ & \\
\hline $2.5-3$ & $26(3.8 \%)$ & \\
\hline$>3$ & $46(11.5 \%)$ & \\
\hline Missing & $127(31.8 \%)$ & \\
\hline
\end{tabular}




\begin{tabular}{|c|c|c|}
\hline (continuation) & $\begin{array}{l}\text { n(\%) or median } \\
\text { (IQR, range) }\end{array}$ & $\begin{array}{r}\text { imputed } \\
n(\%)\end{array}$ \\
\hline \multicolumn{3}{|l|}{ Ki-67 index:* } \\
\hline$<5$ & $202(50.5 \%)$ & \\
\hline$<10$ & $52(13.0 \%)$ & \\
\hline$\geq \mathbf{1 0}$ & $22(5.5 \%)$ & \\
\hline Missing & $124(31.0 \%)$ & \\
\hline Elevated CgA >6x ULN* & $98(24.5 \%)$ & $107(26.8 \%)$ \\
\hline Missing & $88(22.0 \%)$ & \\
\hline Elevated serotonin* & $143(35.8 \%)$ & $163(40.8 \%)$ \\
\hline Missing & $212(53.0 \%)$ & \\
\hline Elevated liver tests* & $33(8.3 \%)$ & $35(8.8 \%)$ \\
\hline Missing & $53(13.3 \%)$ & \\
\hline \multicolumn{3}{|l|}{ Treatment: } \\
\hline \multicolumn{3}{|l|}{ Groups: } \\
\hline Surgery with curative intent & $26(6.5 \%)$ & \\
\hline Surgery with palliative intent & $149(37.3 \%)$ & \\
\hline No surgery & $225(56.3 \%)$ & \\
\hline SSA* & $152(38.0 \%)$ & \\
\hline Surgery & $175(43.8 \%)$ & \\
\hline Liver surgery* & $9(2.3 \%)$ & \\
\hline PRRT/RT & $21(5.3 \%)$ & \\
\hline Embolization & $4(1.0 \%)$ & \\
\hline
\end{tabular}

*nomogram variable; WHO PS: World Health Organisation

Performance Score; G1: grade 1; G2: grade 2; SI: small intestine; UP: unknown primary; $C S$ : carcinoid syndrome; $C H D$ : carcinoid heart disease; CgA: chromogranin A; liver tests: any elevation of alkaline phosphatase, gammaglutyltransferase or bilirubin; ULN: upper limit of normal

ethnicity, which is representative for the Dutch population. In 96 patients (24\%) no primary tumour could be identified, but consensus was reached by the multidisciplinary expert panel on the origin of the tumour. Most patients $(n=267$, $67 \%$ ) experienced functional symptoms. In 24 patients (6\%) CHD was present at time of referral. Over three-quarters of patients $(n=305,76 \%)$ had distant metastases at referral, of whom 236 patients (77\%) had liver metastases.

WHO grade I tumours accounted for the majority of patients $(n=265,66 \%)$, grade II tumours were seen in 94 patients (24\%). In 41 patients (10\%) no distinction could be made between grade I and grade II. Nevertheless, these tumours were recognized as 
well-differentiated low-grade tumours. Tumour size was determined by pathology reports and was available for patients that underwent surgery for their primary tumour $(\mathrm{n}=138,35 \%)$. Tumour size $(\mathrm{cm})$ was $<2,2-2.5,2.5-3$ and $>3$ in 44 patients $(11 \%), 22$ patients $(6 \%), 26$ patients $(4 \%)$ and 46 patients $(12 \%)$, respectively. Approximately half of the patients $(n=202,51 \%$ ) had a Ki-67 index of $<5 \%$; 52 patients (13\%) a Ki-67 index between 5 and $10 \%$, and 22 patients (6\%) a Ki-67 index $\geq 10 \%$. After imputation, 107 patients (27\%) had CgA levels $>6 x$ ULN, 163 patients $(41 \%)$ had elevated serotonin levels, and 35 patients (9\%) had elevated liver tests.

\section{- Primary treatment}

In our cohort, 184 patients (46\%) underwent surgery. Twenty-six patients $(7 \%)$ underwent surgery with curative intent and 149 patients (37\%) underwent surgery with palliative intent. Nine patients $(2 \%)$ underwent liver surgery for metastases. Somatostatin analogues were used by 152 patients (38\%) whereas 21 patients (5\%) were treated with nuclear- or radiotherapy, four patients (1\%) were treated with liver embolization.

\section{- Nomogram survival and actual DSS}

Median follow up time was 5.0 years with a median actual DSS of 9.8 years for patients from the institutional cohort (Figure 1a). At the end of follow-up 80 patients $(20 \%)$ died of their SI-NET, 50 patients $(12.5 \%)$ died of an unknown cause of death, 21 patients $(5.3 \%)$ died of other causes and the remaining 249 patients $(62.3 \%)$ of patients were alive at end of follow-up. Considering the separate strata, median actual DSS was 17.1 in the low-risk group, 9.8 years in the medium-risk group, and 6.8 years in the high-risk group. The nomogram was able to differentiate between low-, medium- and high-risk groups $(\mathrm{P}<0.001$, Figure $1 \mathrm{~b})$.

The predicted nomogram survival compared to the actual DSS for scenario 1 can be found in Figure 2. In this scenario, patients in the low-, medium- and high- risk group had a 5 -year predicted nomogram survival of $86 \%, 52 \%$ and $26 \%$, compared to an actual 5-year DSS of $82 \%, 71 \%$ and $53 \%$, respectively $(\mathrm{P}<0.001)$. The 10 -year predicted nomogram survival was $68 \%, 40 \%$ and $20 \%$ compared to the actual $10-$ year DSS of $69 \%, 50 \%$ and $35 \%$ in the low-, medium- and high- risk group, respectively $(\mathrm{P}<0.001)$. Similar significant differences in DSS were seen for 


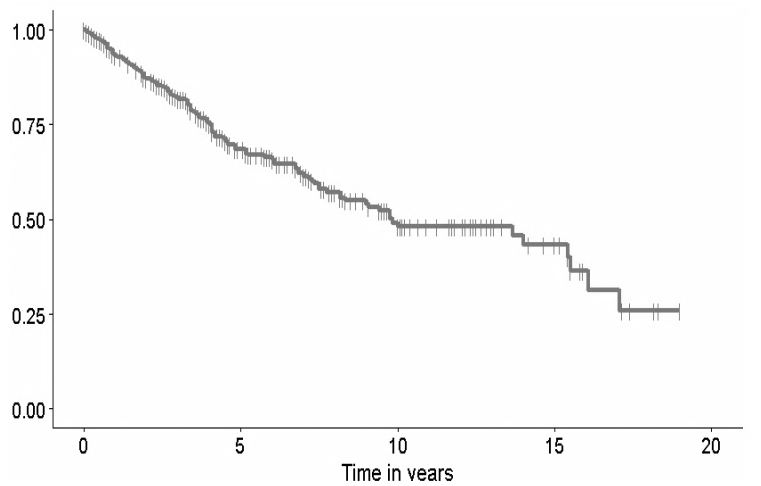

Figure 1a: Disease specific survival curve of the institutional cohort

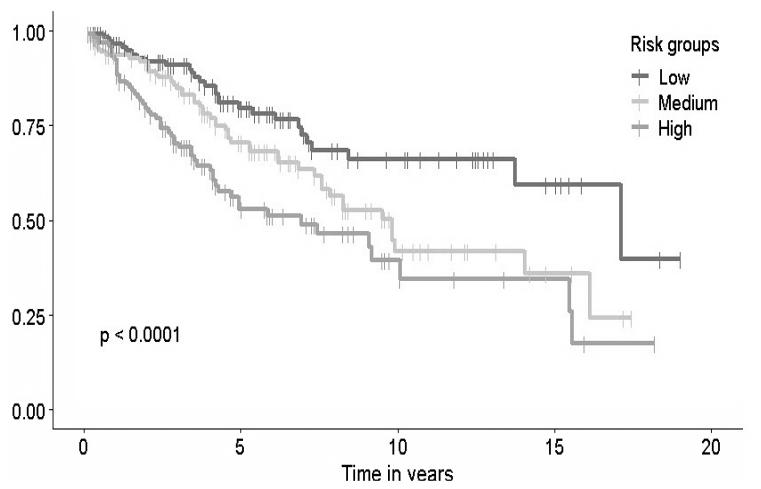

Figure 1b: Disease specific survival curves for low-, medium-, and high-risk strata

scenario 2 and in the different treatment subgroups. The nomogram overestimated 5year DSS in the low risk group, but underestimated DSS in all other groups. The predicted nomogram survival for scenario 1 and 2 and actual DSS divided by subgroups can be found in the Supplementary Material (Table S1 and S2).

The difference between the predicted nomogram survival and actual DSS ranged from 1 to $46 \%$. The median difference was 24\% (IQR 16-39) for 5-year survival and $20 \%$ (IQR 10-28) for 10-year survival. For all scenario's and treatment groups the difference in predicted nomogram survival and actual DSS was the smallest for patients in the low-risk group (median difference in survival 20\%, IQR 1-29), compared to medium-risk group (median difference in survival 22\%, IQR 17-44) and high-risk group (median difference in survival 24\%, IQR 9-36). 

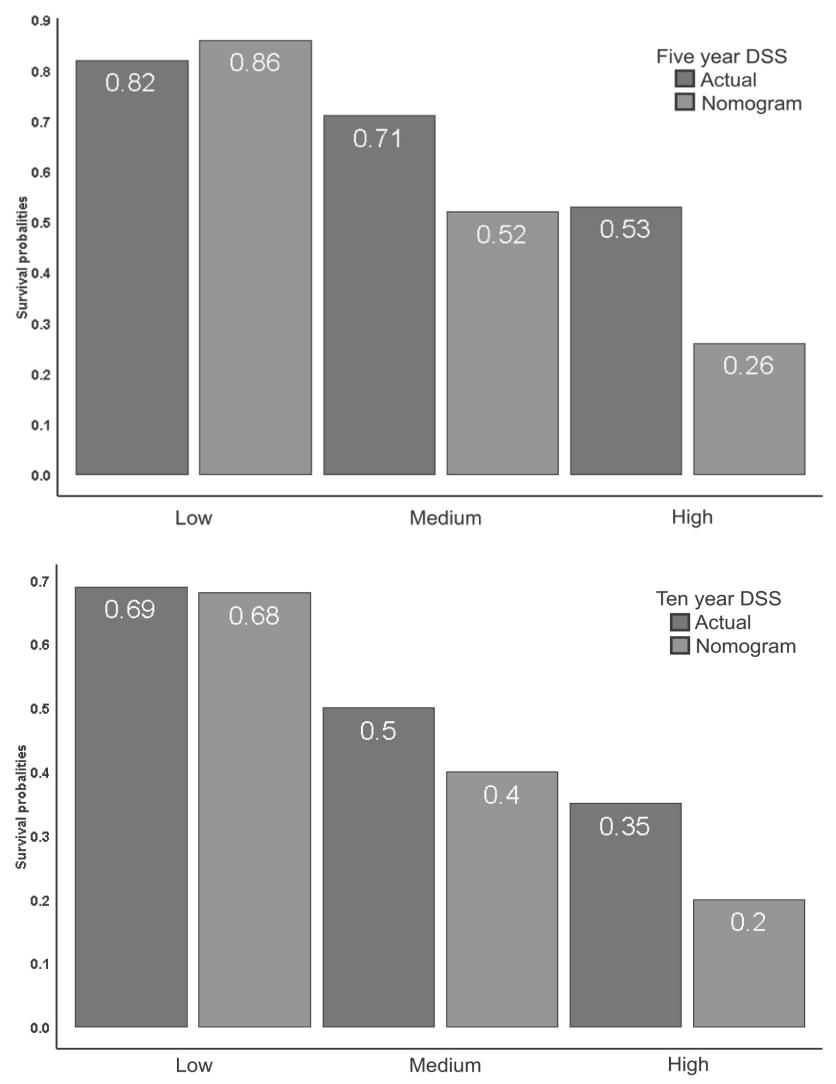

Figure 2: Actual DSS compared to nomogram surival

- Prognostic indicators

Univariable analysis identified age (HR 1.06), WHO performance score of 2 (HR 4.0 ), Ki-67 index $\geq 10 \%$ (HR 1.3), grade 2 tumours (HR 1.8), an unknown primary tumour (HR 1.3), distant (HR 2.2) and liver metastases (HR 2.5), the presence of CS (HR 1.7) and CHD (HR 2.3), elevated CgA >6 ULN (HR 4.3) and any elevated liver test (HR 4.9) to be associated with actual DSS (Table 2). In multivariable analysis age (HR 1.07), WHO performance status of 2 (HR 4.4), an unknown primary tumour (HR 3.2), Ki-67 index $\geq 10 \%$ (HR 12.6), CgA $>6$ times ULN (HR 3.2) and elevated liver tests (HR 3.1) remained independent predictors for DSS in both the imputed as well as the non-imputed dataset (Table 3 ). 
Part I | Chapter 2

Table 2: Univariable analysis for disease specific survival

\begin{tabular}{|c|c|c|c|}
\hline Variable & HR & $95 \% \mathrm{Cl}$ & $P$ \\
\hline Age & 1.06 & $1.04-1.08$ & 0.001 \\
\hline Gender & 0.72 & $0.51-1.02$ & 0.149 \\
\hline \multicolumn{4}{|l|}{ Etnicity: } \\
\hline Caucasian & 1 & & \\
\hline Black & 1.11 & $0.27-4.55$ & 0.882 \\
\hline Other & 1.01 & $0.25-4.712$ & 0.992 \\
\hline \multicolumn{4}{|l|}{ WHO PS: } \\
\hline $\mathbf{0}$ & 1 & & \\
\hline 1 & 1.72 & $1.11-2.67$ & 0.014 \\
\hline 2 & 4.03 & $2.27-7.14$ & 0.001 \\
\hline \multicolumn{4}{|l|}{ Grade: } \\
\hline 1 & 1 & & \\
\hline 2 & 1.83 & $1.23-2.74$ & 0.003 \\
\hline \multicolumn{4}{|c|}{ Ki-67 index: } \\
\hline G1 & 1 & & \\
\hline \multicolumn{4}{|l|}{ G2: } \\
\hline$<5$ & 1.00 & $0.46-2.16$ & 0.994 \\
\hline$<10$ & 1.28 & $0.69-2.36$ & 0.429 \\
\hline$\geq 10$ & 4.0 & $2.16-7.40$ & 0.001 \\
\hline
\end{tabular}

Primary tumour:

\begin{tabular}{|c|c|c|c|}
\hline \multirow{2}{*}{$\begin{array}{l}\text { SI } \\
\text { Unknown primary }\end{array}$} & \multicolumn{2}{|l|}{1} & \multirow[b]{2}{*}{0.001} \\
\hline & 1.34 & $1.23-1.46$ & \\
\hline Distant metastases & 2.23 & $1.39-3.68$ & 0.001 \\
\hline Liver metastases & 2.51 & $1.70-3.70$ & 0.001 \\
\hline CS & 1.72 & $1.13-2.60$ & 0.011 \\
\hline CHD & 2.27 & $1.34-3.83$ & 0.002 \\
\hline CgA >6x ULN & 4.28 & $2.83-6.49$ & 0.001 \\
\hline Serotonin $>$ ULN & 1.07 & $0.59-1.94$ & 0.814 \\
\hline Liver tests $>$ ULN & 4.94 & $2.92-8.36$ & 0.001 \\
\hline \multicolumn{4}{|c|}{$\begin{array}{l}\text { WHO PS: World Health Organisation Performance Score; G1: grade 1; } \\
\text { G2: grade 2; SI: small intestine; UP: unknown primary; CS: carcinoid } \\
\text { syndrome; CHD: carcinoid heart disease; CgA: elevated chromogranin } \\
\text { A; liver function tests: any elevation of alkaline phosphatase; } \\
\text { gammaglutyltransferase or bilirubin; ULN: upper limit of normal }\end{array}$} \\
\hline
\end{tabular}


Table 3: Multivariable analysis for disease specific survival of the institutional cohort

\begin{tabular}{|c|c|c|c|c|c|c|}
\hline \multirow[b]{2}{*}{ Variable } & \multicolumn{3}{|c|}{ Original dataset } & \multicolumn{3}{|c|}{ Imputed dataset } \\
\hline & HR & $95 \% \mathrm{Cl}$ & $\boldsymbol{P}$ & HR & $95 \% \mathrm{CI}$ & $P$ \\
\hline Age & 1.07 & $1.04-1.10$ & 0.001 & 1.07 & 1.04-1.09 & 0.001 \\
\hline \multicolumn{7}{|c|}{ WHO PS: } \\
\hline $\mathbf{0}$ & 1 & & & 1 & & \\
\hline 1 & 1.11 & $0.58-2.14$ & 0.756 & 1.13 & $0.62-2.07$ & 0.676 \\
\hline 2 & 4.40 & $2.09-9.20$ & 0.001 & 4.14 & $2.01-8.51$ & 0.001 \\
\hline \multicolumn{7}{|c|}{ Ki-67 index } \\
\hline G1 & 1 & & & 1 & & \\
\hline \multicolumn{7}{|l|}{ G2: } \\
\hline$<5$ & 2.38 & $0.79-7.11$ & 0.121 & 2.51 & $0.94-6.73$ & 0.067 \\
\hline$<10$ & 1.04 & $0.46-2.36$ & 0.918 & 0.99 & $0.44-2.22$ & 0.981 \\
\hline$\geq 10$ & 12.61 & $5.51-28.84$ & 0.001 & 11.56 & $5.15-25.93$ & 0.001 \\
\hline
\end{tabular}

Primary tumour:

\begin{tabular}{lrrrrrr} 
SI & 1 & \multicolumn{5}{c}{1} \\
UP & 3.24 & $1.42-4.74$ & 0.001 & 2.32 & $1.32-4.01$ & 0.003 \\
\hline CgA >x ULN & 3.24 & $1.91-5.46$ & 0.001 & 3.43 & $2.06-5.70$ & 0.001 \\
Liver tests > ULN & 3.10 & $1.45-6.63$ & 0.004 & 3.12 & $1.46-6.60$ & 0.003
\end{tabular}

WHO PS: World Health Organisation Performance Score; G1: grade 1; G2: grade 2; SI: small intestine; UP: unknown primary; CgA: elevated chromogranin A; liver tests: any elevation of alkaline phosphatase; gammaglutyltransferase or bilirubin. ULN: upper limit of normal

\section{Discussion}

In this study a previously designed nomogram by Modlin et al., based on clinical and tumour characteristics, identified low-, medium- and high- risk groups in patients with SI-NETs. However, 5- and 10-year survival was generally underestimated. The nomogram was best in predicting 5 -year disease specific survival for patients who were predicted to be at low risk. In our population, in multivariable analyses age, a WHO performance status of 2, an unknown primary tumour, Ki-67 index $\geq 10 \%$, elevated CgA >6x ULN, and elevated liver tests were independent predictors for a worse DSS. These factors could contribute to further improvement of a prognostic model. 
Patients with SI-NETs have a very heterogeneous disease course. Despite the identification of several prognostic factors, predicting prognosis for an individual patient with SI-NET remains challenging. ${ }^{19,24}$ Like in other malignancies, a more individual approach in the treatment of patients with a SI-NET is desired.

Predicting the individual survival by a nomogram based on clinical and tumour characteristics could aid in optimization of personalized cancer treatment. However, validation of any developed nomogram is vital before it can be routinely used.

The evaluated nomogram is based on a large dataset from 7.455 patients from the SEER database and variables were selected and weighed after extensive analyses of literature-curated data. However, the nomogram itself was initially validated in only 33 patients. Two earlier studies have attempted to validate the predictive properties of the SI-NET nomogram. Clift et al. (2017) showed that the predicted nomogram DSS matched the observed 5-year and 10-year DSS in a cohort of 70 patients. ${ }^{31}$ This difference between our cohorts is not easily explained, our populations were quite comparable with regard to the baseline characteristics. However, patients were more often treated with PRRT (21\% compared to 5\% in our cohort), which suggests that their patients might have had more extensive disease burden.

In the study performed by Kelly et al. (2019), the nomogram was able to predict survival of patients in a cohort of 121 patients who underwent surgery with curative intent. ${ }^{18}$ However, the nomogram score was not identified as an independent predictor for survival in multivariable analysis. The authors argued that this might be explained by their high survival rate of patients at the end of their follow up period (90.9\%). Similarly, our patients had a significantly higher DSS than was estimated by the nomogram. Of note, we calculated DSS in 39\% of the patients from time to referral to obtain a high quality dataset, this might have reduced the observed difference. Nevertheless, both Clift et al. and Kelly et al. recognized the prognostic potential of a nomogram based on clinical and tumour characteristics, with the need for extensive validation and possibly improvement. Subsequently, Kelly et al. recently developed a new nomogram based for patients specifically from the United States. ${ }^{36}$

Several studies have investigated survival of patients with NET over different time periods and found that survival increases in patients diagnosed in more recent time periods. ${ }^{1,4}$ This is primarily due to new systemic treatment modalities that have emerged which have a beneficial effect on NET-related survival. SSAs made an 
entrance in 1987 and was the first systemic treatment option specifically for NET. Initially, SSAs were found to reduce symptoms of carcinoid syndrome, but an antiproliferative effect was shown in the PROMID and CLARINET study. ${ }^{32-34}$ PRRT was introduced in 2008. This treatment uses a radiolabeled somatostatin analogue to achieve local intratumoural nuclear therapy and showed a survival benefit for patients with a SI-NET treated with PRRT in the NETTER-1 study. ${ }^{35}$ Additionally, diagnostic techniques for NETs have improved, earlier detection could contribute to an improved survival. ${ }^{4}$ Likewise, the underestimation of survival by the nomogram could be explained by the fact that the nomogram was based on studies from 1997 and 2010 and on SEER data from patients diagnosed between 1977-2007. As a consequence, the nomogram was probably based on a poorer survival outcome compared to our cohort.

We found that a Ki-67 index $\geq 10 \%$ was associated with a worse DSS. This cut-off value is currently not used in clinical practice. The cut-off point most suited for distinguishing between grade I and II NET has been the subject of debate since the introduction of the Ki-67 index in the ENETS grading system in 2007. In a systematic review by Richards-Taylor et al. it was postulated that grade II and $\mathrm{Ki}-67$ index $<5 \%$ NET was more similar to grade I NET, compared to a grade II NET with Ki-67 index $>5 \%$. ${ }^{37}$ Although in our cohort, a Ki-67 index $<5 \%$, or Ki-67 index $<10 \%$ could not be identified as a separate prognostic factor for DSS, it does support the notion that the current Ki-67 index subdivision used for grading SI-NETs might be insufficient for adequate prognostication. Future studies should aim to determine which cut-off values, if any, albeit in combination with other histopathological characteristics, would be more suitable.

An elevated CgA at referral of $>6 x$ ULN was associated with a shorter DSS. Several studies have shown the prognostic value of baseline CgA as well. ${ }^{38}$ This is in line with previous studies indicating that $\mathrm{CgA}$ is a marker of bulky disease, which is associated with poor survival. ${ }^{39}$ Others have discussed that a change of CgA (of 25$20 \%$ ) might be a better prognostic predictor than a single measurement however this needs further validation. ${ }^{39,40}$ Currently, there is a debate on the prognostic potential of $\mathrm{CgA}$, since $\mathrm{CgA}$ is often within the normal range also in patients with metastatic disease (unpublished data). Although there might be room for improvement to decide the optimal measurement. ${ }^{27}$ 
Remarkably, liver metastases did not prove to be an independent prognostic predictor for DSS. Many other studies did show this association. ${ }^{7,41-44}$ However, we included an unknown primary tumour as a separate variable in our multivariable analysis, which likely influenced this outcome since it overlaps with the presence of (liver) metastases. Yet, any elevation of liver test was associated with a worse DSS. It supports the notion that the extent of liver metastases (resulting in elevated liver test), might be more important than the presence of liver metastases alone. ${ }^{45,46}$

A major strength of this study is the large patient population with detailed data on treatment and DSS. Moreover, our patient population treated in an ENETS CoE entails a representative patient population with a considerable follow up period incorporating all available therapies for patients with SI-NETs. Furthermore, we calculated the predicted nomogram survival and actual DSS for both best and worst case scenarios and included patients from all possible treatment categories. Additionally, to avoid confounding by indication, surgical treatment was excluded from analysis, since the policy in our centre is to perform a resection if technically achievable with either curative or palliative intention. Instead, nomogram survival and actual DSS was compared in different treatment groups. This ensures that the nomogram was evaluated in all possible real-world and clinically relevant patient groups, and assessed for its clinical validity in daily clinical practice.

Nevertheless, several limitations should be taken into consideration. Unfortunately, the included biomarkers, were not always available because of changes in clinical practice over time. For example, CgA was measured from 2004, simultaneously abandoning urinary 5-HIAA as a biomarker, while serotonin in thrombocytes was introduced in 2010. However, the variable use of biomarkers is consistent with other studies in NET. ${ }^{11,28}$ Another downside of a retrospective cohort study is missing data. This is inherent to collecting data in a longitudinal clinical database compared to data collection within the framework of a clinical trial. We handled missing data with the use of imputation. Analyses were performed with an imputed and nonimputed dataset. For missing and non-imputed data, a best and worst case scenario was calculated assigning no points or the highest points possible. In both the imputed as non-imputed dataset and in various scenario's our results were highly comparable. This suggests that, despite the retrospective character, our results are robust.

On the whole, while nomograms can fulfil an important role in personalized cancer care when it accurately models clinical outcome ${ }^{47}$, the nomogram in its current form 
unfortunately seems insufficient to accurately predict individual prognosis. Recent advances in identifying (molecular) prognostic factors and the development of liquid biopsies such as CTC or circulating tumour transcripts (the NETest ${ }^{\circledR}$ ), could be a valuable addition for individualized prognostication. ${ }^{48}$ Prognostic studies often focussed either solely on clinical and tumour characteristics ${ }^{1,4,6,7,10}$ or the sole predictive value of a biomarker alone (subsequently abandoning the use of clinical and tumour characteristics)..$^{27,28,48}$ Our study confirms the prognostic potential of a nomogram based on clinical and tumour characteristics while underlining the need for improvement. Future studies should aim to combine clinical and tumour prognostic factors with potential new (molecular) biomarkers. By doing so, an advanced method of prognostic modelling for individual patients could be achieved.

\section{Conclusion}

A nomogram based on clinical data and tumour characteristics was the first extensive attempt for individual prognostication. The nomogram was able to differentiate between survival for patients in the low, medium and high risk groups. However, the nomogram generally underestimated. Age, WHO performance status of 2 , an unknown primary tumour, Ki-67 index $\geq 10 \%$, elevated $\mathrm{CgA}>6 \mathrm{x}$ ULN, and elevated liver tests were identified as independent predictors for a worse DSS. Our findings suggest that improvement of individualized estimation of prognosis is desirable. Future studies should aim to combine clinical and tumour prognostic factors with potential new (molecular) biomarkers. 


\section{References}

1. Yao JC, Hassan M, Phan A, et al. One hundred years after "carcinoid": epidemiology of and prognostic factors for neuroendocrine tumors in 35,825 cases in the United States. Journal of clinical oncology : official journal of the American Society of Clinical Oncology 2008;26:3063-72.

2. Hallet J, Law $\mathrm{CH}$, Cukier $\mathrm{M}$ et al. Exploring the rising incidence of neuroendocrine tumors: a population-based analysis of epidemiology, metastatic presentation, and outcomes. Cancer 2015;121:589-97.

3. Garcia-Carbonero R, P JI-F, Teule A, et al. Spanish Society for Medical O. SEOM clinical guidelines for the diagnosis and treatment of gastroenteropancreatic neuroendocrine neoplasms (GEP-NENs) 2014. Clinical \& translational oncology : official publication of the Federation of Spanish Oncology Societies and of the National Cancer Institute of Mexico 2014;16:1025-34.

4. Dasari A, Shen C, Halperin D, et al. Trends in the Incidence, Prevalence, and Survival Outcomes in Patients With Neuroendocrine Tumors in the United States. JAMA oncology 2017;3:1335.

5. Pan SY, Morrison H. Epidemiology of cancer of the small intestine. World journal of gastrointestinal oncology 2011;3:33-42.

6. Boyar Cetinkaya R, Aagnes B, et al. Survival in neuroendocrine neoplasms; A report from a large Norwegian population-based study. International journal of cancer Journal international du cancer 2018;142:1139-47.

7. Lesen E, Granfeldt D, Berthon A, et al. Treatment Patterns and Survival among Patients with Metastatic Gastroenteropancreatic Neuroendocrine Tumours in Sweden - a Population-based Register-linkage and Medical Chart Review Study. Journal of Cancer 2019;10:6876-87.

8. Korse $T$, Taal B, van Velthuysen $M$, et al. Incidence and survival of neuroendocrine tumours in the Netherlands according to histological grade: Experience of two decades of cancer registry. European Journal of Cancer 2013;49:1975-83.

9. Fisher AT, Titan AL, Foster DS, et al. Management of Ileal Neuroendocrine Tumors with Liver Metastases. Journal of gastrointestinal surgery : official journal of the Society for Surgery of the Alimentary Tract 2019.

10. Shah CP, Mramba LK, Bishnoi R,et al. Survival trends of metastatic small intestinal neuroendocrine tumor: a population-based analysis of SEER database. Journal of gastrointestinal oncology 2019;10:869-77.

11. Larouche V, Akirov A, Alshehri S, et al. Management of Small Bowel Neuroendocrine Tumors. Cancers 2019;11(9):1395. 
12. Rossi RE, Massironi S, Spampatti MP, et al. Treatment of liver metastases in patients with digestive neuroendocrine tumors. Journal of gastrointestinal surgery : official journal of the Society for Surgery of the Alimentary Tract 2012;16:1981-92.

13. Frilling A, Clift AK. Therapeutic strategies for neuroendocrine liver metastases. Cancer 2015;121:1172-86.

14. Kollar A, Butikofer L, Ochsenbein A, et al. Treatment sequence in patients with neuroendocrine tumours: a nationwide multicentre, observational analysis of the Swiss neuroendocrine tumour registry. Swiss medical weekly 2020;150:w20176.

15. Kaderli RM, Spanjol M, Kollar A, et al. Therapeutic Options for Neuroendocrine Tumors: A Systematic Review and Network Meta-analysis. JAMA oncology 2019;5:480-9.

16. Landerholm K, Zar N, Andersson RE, et al. Survival and prognostic factors in patients with small bowel carcinoid tumour. The British journal of surgery 2011;98:1617-24.

17. Mocellin S, Nitti D. Gastrointestinal carcinoid: epidemiological and survival evidence from a large population-based study $(n=25531)$. Annals of Oncology 2013;24:3040-4.

18. Kelly S, Aalberg J, Agathis A, et al. Predicting Survival of Small Intestine Neuroendocrine Tumors: Experience From a Major Referral Center. Pancreas 2019;48:514-8.

19. Nunez-Valdovinos B, Carmona-Bayonas A, Jimenez-Fonseca P, et al. Neuroendocrine Tumor Heterogeneity Adds Uncertainty to the World Health Organization 2010 Classification: Real-World Data from the Spanish Tumor Registry (R-GETNE). The oncologist 2018;23:422-32.

20. Modlin IM, Gustafsson BI, Pavel M,et al. A nomogram to assess small-intestinal neuroendocrine tumor ('carcinoid') survival. Neuroendocrinology 2010;92:143-57.

21. Karpathakis A, Dibra H, Pipinikas C, et al. Prognostic Impact of Novel Molecular Subtypes of Small Intestinal Neuroendocrine Tumor. Clinical Cancer Research 2015;22:250-8.

22. Modlin IM, Lye KD, Kidd M. A 5-decade analysis of 13,715 carcinoid tumors. Cancer 2003;97:934-59.

23. Samsom KG, van Veenendaal LM, Valk GD, et al. Molecular prognostic factors in small-intestinal neuroendocrine tumours. Endocr Connect. 2019;8(7):906 - 922.

24. Oberg K, Krenning E, Sundin A. A Delphic consensus assessment: imaging and biomarkers in gastroenteropancreatic neuroendocrine tumor disease management. Endorine Connections 2016;5:174-87. 
25. Pavel M, Jann H, Prasad V, et al. NET Blood Transcript Analysis Defines the Crossing of the Clinical Rubicon: When Stable Disease Becomes Progressive.

Neuroendocrinology 2017;104:170-82.

26. Oberg K, Califano A, Strosberg JR, et al. A meta-analysis of the accuracy of a neuroendocrine tumor mRNA genomic biomarker (NETest) in blood. Annals of oncology : official journal of the European Society for Medical Oncology / ESMO 2020;31:202-12.

27. van Treijen MJC, Korse CM, van Leeuwaarde RS, et al. Blood Transcript Profiling for the Detection of Neuroendocrine Tumors: Results of a Large Independent Validation Study. Frontiers in endocrinology 2018;9:740.

28. Modlin IM, Kidd M, Malczewska A, et al. The NETest: The Clinical Utility of Multigene Blood Analysis in the Diagnosis and Management of Neuroendocrine Tumors. Endocrinology and metabolism clinics of North America 2018;47:485-504.

29. Ross RW, Galsky MD, Scher HI, et al. A whole-blood RNA transcript-based prognostic model in men with castration-resistant prostate cancer: a prospective study. Lancet Oncol. 2012;13:1105-13.

30. Kuiper R, Duin MV, Vliet MHV, et al. Prediction of high- and low-risk multiple myeloma based on gene expression and the International Staging System. Blood. 2015;126:1996-2004

31. Clift AK, Faiz O, Goldin R, et al. Predicting the survival of patients with small bowel neuroendocrine tumours: comparison of 3 systems. Endocrine connections 2017;6:71-81.

32. Rinke A, Müller HH, Schade-Brittinger C, et al. Placebo-controlled, double-blind, prospective, randomized study on the effect of octreotide LAR in the control of tumor growth in patients with metastatic neuroendocrine midgut tumors: A report from the PROMID study group. 2009;27:4656-63.

33. Rinke A, Wittenberg M, Schade-Brittinger C, et al. Placebo Controlled, Double Blind, Prospective, Randomized Study on the Effect of Octreotide LAR in the Control of Tumor Growth in Patients with Metastatic Neuroendocrine Midgut Tumors (PROMID): Results on Long Term Survival. Neuroendocrinology 2017;104(1):26-32.

34. Caplin ME, Pavel M, Ćwikła JB, et al. Lanreotide in metastatic enteropancreatic neuroendocrine tumors. 2014;371:224-33.

35. Strosberg J, El-Haddad G, Wolin E et al. Phase 3 trial of $177 \mathrm{Lu}$-dotatate for midgut neuroendocrine tumors. N Engl J Med 2017;376(2):125-135.

36. Kelly S, Aalberg J, Kim MK, et al. A Predictive Nomogram for Small Intestine Neuroendocrine Tumors. Pancreas 2020;49:524-8. 
37. Richards-Taylor S, Ewings SM, Jaynes E, et al. The assessment of Ki-67 as a prognostic marker in neuroendocrine tumours: a systematic review and meta-analysis. Journal of clinical pathology 2016;69:612-8.

38. Yao JC, Pavel M, Lombard-Bohas C, et al. Everolimus for the Treatment of Advanced Pancreatic Neuroendocrine Tumors: Overall Survival and Circulating Biomarkers From the Randomized, Phase III RADIANT-3 Study. Journal of Clinical Oncology 2016;10;34(32):3906-3913.

39. Chou WC, Chen JS, Hung YS, et al. Plasma Chromogranin A Levels Predict Survival and Tumor Response in Patients with Advanced Gastroenteropancreatic Neuroendocrine Tumors. Anticancer research 2014;34:5661-70.

40. Jensen $\mathrm{KH}$, Hilsted L, Jensen $\mathrm{C}$, et al. Chromogranin A is a sensitive marker of progression or regression in ileo-cecal neuroendocrine tumors. Scandinavian journal of gastroenterology 2013;48:70-7.

41. Pape UF, Berndt U, Muller-Nordhorn J, et al. Prognostic factors of long-term outcome in gastroenteropancreatic neuroendocrine tumours. Endocrine-related cancer 2008;15:1083-97.

42. Carmona-Bayonas A. Prediction of Progression-Free Survival in Patients With Advanced, Well-Differentiated, Neuroendocrine Tumors Being Treated With a Somatostatin Analog: The GETNE-TRASGU Study. Journal of Clinical Oncology $2019 ; 37$.

43. Laskaratos FM, Walker M, Wilkins D, et al. Evaluation of clinical prognostic factors and further delineation of the effect of mesenteric fibrosis on survival in advanced midgut neuroendocrine tumours. Neuroendocrinology 2018.

44. Laskaratos FM, Walker M, Naik K, et al. Predictive factors of antiproliferative activity of octreotide LAR as first-line therapy for advanced neuroendocrine tumours. British journal of cancer 2016;115:1321-7.

45. Manguso N. Prognostic Factors Associated with Outcomes in SmallBowel Neuroendocrine Tumors. The American surgeon 2017;83:1174-8.

46. Manguso N, Nissen N, Hendifar A, et al. Prognostic factors influencing survival in small bowel neuroendocrine tumor with liver metastases. J Surg Oncol.

2019;120(6):926-931

47. Balachandran VP, Gonen M, Smith JJ, et al. Nomograms in oncology: more than meets the eye. Lancet Oncol. 2015;16(4):e173-80.

48. Liu E, Paulson S, Gulati A, et al. Assessment of NETest Clinical Utility in a U.S. Registry-Based Study. Oncologist. 2019;24(6):783-790. 
Part I | Chapter 2

Supplementary Table S1: Actual disease specific survival and nomogram survival

\begin{tabular}{lcccccc} 
Stratum & $\begin{array}{c}\text { Actual } \\
\text { 5-year DSS }\end{array}$ & $\begin{array}{c}\text { Nomogram } \\
\text { 5-year DSS }\end{array}$ & $\boldsymbol{P}$ & $\begin{array}{c}\text { Actual } \\
\text { 10-year DSS }\end{array}$ & $\begin{array}{c}\text { Nomogram } \\
\text { 10-year DSS }\end{array}$ & $\boldsymbol{P}$ \\
\hline \hline Low & 0.82 & 0.86 & $<0.001$ & 0.69 & 0.68 & $<0.001$ \\
Medium & 0.71 & 0.52 & $<0.001$ & 0.50 & 0.40 & $<0.001$ \\
High & 0.53 & 0.26 & $<0.001$ & 0.35 & 0.20 & $<0.001$ \\
Low & 0.89 & 0.64 & $<0.001$ & 0.79 & 0.50 & $<0.001$ \\
Medium & 0.70 & 0.25 & $<0.001$ & 0.46 & 0.20 & $<0.001$ \\
High & 0.47 & 0.08 & $<0.001$ & 0.29 & 0.08 & $<0.001$
\end{tabular}

Stratum: column indicating low-, medium- or high risk groups based on nomogram scores; $5 Y$ DSS: five year disease specific survival, 10Y DSS: 10-year disease specific survival 


\section{Supplementary S2: Actual disease specific survival and nomogram survival in the treatment groups}

\begin{tabular}{|c|c|c|c|c|c|c|}
\hline Stratum & $\begin{array}{c}\text { Actual } \\
\text { 5-year DSS }\end{array}$ & $\begin{array}{l}\text { Nomogram } \\
5 \text {-year DSS }\end{array}$ & $\boldsymbol{P}$ & $\begin{array}{c}\text { Actual } \\
\text { 10-year DSS }\end{array}$ & $\begin{array}{l}\text { Nomogram } \\
\text { 10-year DSS }\end{array}$ & $\boldsymbol{P}$ \\
\hline \multicolumn{7}{|c|}{ Scenario 1} \\
\hline \multicolumn{7}{|c|}{ Curative surgery } \\
\hline Low & 0.96 & 0.88 & $<0.001$ & $1.0^{*}$ & 0.70 & $<0.001$ \\
\hline Medium & $1.0^{*}$ & 0.56 & $<0.001$ & $1.0^{*}$ & 0.43 & $<0.001$ \\
\hline High & $1.0^{*}$ & 0.33 & $<0.001$ & 0.67 & 0.23 & $<0.001$ \\
\hline \multicolumn{7}{|c|}{ Palliative surgery } \\
\hline Low & 0.82 & 0.86 & $<0.001$ & 0.78 & 0.68 & $<0.001$ \\
\hline Medium & 0.67 & 0.49 & $<0.001$ & 0.46 & 0.38 & $<0.001$ \\
\hline High & 0.50 & 0.26 & $<0.001$ & 0.33 & 0.20 & $<0.001$ \\
\hline \multicolumn{7}{|c|}{ No surgery } \\
\hline Low & 0.81 & 0.85 & $<0.001$ & 0.69 & 0.68 & $<0.001$ \\
\hline Medium & 0.68 & 0.52 & $<0.001$ & 0.49 & 0.40 & $<0.001$ \\
\hline High & 0.50 & 0.26 & $<0.001$ & 0.36 & 0.19 & $<0.001$ \\
\hline \multicolumn{7}{|c|}{ Scenario 2} \\
\hline \multicolumn{7}{|c|}{ Curative surgery } \\
\hline Low & 0.93 & 0.69 & $<0.001$ & $1.0^{*}$ & 0.54 & $<0.001$ \\
\hline Medium & $1.0^{*}$ & 0.23 & $<0.001$ & $1.0^{*}$ & 0.16 & $<0.001$ \\
\hline High & $1.0^{*}$ & 0.08 & $<0.001$ & 0.67 & 0.08 & $<0.001$ \\
\hline \multicolumn{7}{|c|}{ Palliative surgery } \\
\hline Low & 0.83 & 0.67 & $<0.001$ & 0.78 & 0.53 & $<0.001$ \\
\hline Medium & 0.67 & 0.24 & $<0.001$ & 0.42 & 0.18 & $<0.001$ \\
\hline High & 0.50 & 0.08 & $<0.001$ & 0.36 & 0.08 & $<0.001$ \\
\hline \multicolumn{7}{|c|}{ No surgery } \\
\hline Low & 0.91 & 0.59 & $<0.001$ & 0.74 & 0.45 & $<0.001$ \\
\hline Medium & 0.69 & 0.23 & $<0.001$ & 0.36 & 0.18 & $<0.001$ \\
\hline High & 0.45 & 0.08 & $<0.001$ & 0.30 & 0.08 & $<0.001$ \\
\hline
\end{tabular}

Stratum: column indicating low-, medium- or high risk groups based on nomogram scores. 5Y DSS: 5year disease specific survival, $10 Y$ DSS: 5-year disease specific survival. *: no events in group 


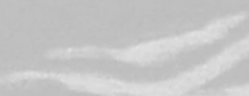

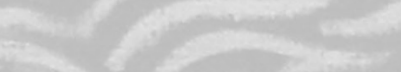

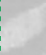

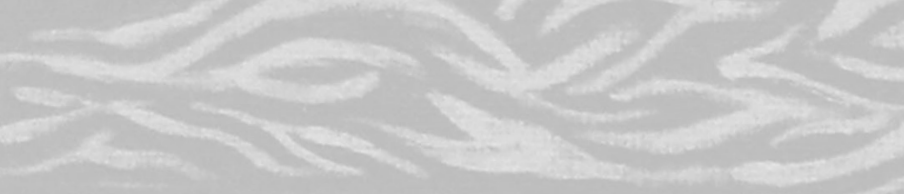




\section{Chapter 3}

\section{Molecular prognostic factors in small-intestinal neuroendocrine tumours}

K.G. Samsom, L.M. van Veenendaal, G.D. Valk, M.R. Vriens, M.E.T. Tesselaar, J.G. van den Berg

Endocrine Connections, 2019 


\begin{abstract}

\section{Background}

Small intestinal neuroendocrine tumours (SI-NETs) represent a heterogeneous group of rare tumours. In recent years, basic research in SI-NETs has attempted to unravel the molecular events underlying SI- NET tumorigenesis.
\end{abstract}

\title{
Aim
}

We aim to provide an overview of the current literature regarding prognostic and predictive molecular factors in patients with SI-NETs.

\section{Method}

A PubMed search was conducted on (epi)genetic prognostic factors in SI-NETs from 2000 until 2019.

\section{Results}

The search yielded 1522 articles of which 18 reviews and 35 original studies were selected for further evaluation. SI-NETs are mutationally quiet tumours with a different genetic make-up compared to pancreatic NETs. Loss of heterozygosity at chromosome 18 is the most frequent genomic aberration (44-100\%) followed by mutations of CDKN1B in 8\%. Prognostic analyses were performed in 16 studies, of which 8 found a significant (epi)genetic association for survival or progression. Loss of heterozygosity at chromosome 18, gains of chromosome 4, 5, 7, 14 and 20p, copy gain of the SRC gene and low expression of RASSF1A and P16 were associated with poorer survival. In comparison with genetic mutations, epigenetic alterations are significantly more common in SI-NETs and may represent more promising targets in the treatment of SI-NETs.

\section{Conclusion}

SI-NETs are mutationally silent tumours. No biomarkers have been identified yet that can easily be adopted into current clinical decision-making. SI-NETs may represent a heterogeneous disease and larger international studies are warranted to translate molecular findings into precision oncology. 


\section{Introduction}

Well-differentiated neuroendocrine tumours (NETs) represent a heterogeneous group of rare tumours, which have a relatively indolent disease course. Primary NETs can arise from neuroendocrine cells at various anatomic sites. They most commonly develop in the gastrointestinal tract and bronchopulmonary system..$^{1-3}$ NETs can be classified as functional or non-functional, based on whether they cause clinical symptoms as a result of hormone secretion or not. In patients with metastatic smallintestinal NETs (SI-NETs), the carcinoid syndrome is common, which is characterised by diarrhoea, episodic flushing, bronchospasm and often carcinoid heart disease leading to right valvular dysfunction. ${ }^{4}$ Patients with non-functional SI-NETs are often asymptomatic or experience non-specific symptoms resulting in metastatic disease at the time of diagnosis in $27-73 \%$ of patients. ${ }^{1-3}$ In contrast to pancreatic NETs, SINETs are not known to arise in the context of hereditary syndromes, for example multiple endocrine neoplasia (MEN) type 1 or 2 and Von Hippel Lindau disease.

The reported incidence of SI-NETs has increased over the last four decades, from 0.2 per 100.000 individuals in 1973 to 1.25 per 100.000 individuals in $2012 .^{5}$ This progressive rise can mainly be contributed to more frequent use and improvements of diagnostic modalities or alterations in pathological disease definition. ${ }^{2,5}$ In the group of gastroenteropancreatic NETs, SI-NETs are second most prevalent after rectum NETs and followed by pancreatic NETs. ${ }^{5}$ Moreover, SI-NETs are the most frequent cancer type of the small intestine. ${ }^{6}$

Currently, treatment for patients with SI-NETs is based on the availability of several treatment modalities, for example, surgery, liver-directed therapies, somatostatin receptor analogues and peptide receptor radionuclide therapy rather than on precision medicine. In case of non-functional, advanced and progressive SI-NETs, everolimus, targeting the $\mathrm{P} 13 \mathrm{~K} / \mathrm{AKT} / \mathrm{mTOR}$ (mammalian target of rapamycin) pathway, has demonstrated anti-proliferative effects..$^{7-9}$ However, there is no biomarker available that predicts response to everolimus. To conclude, personalised treatment based on molecular profiling has not yet entered the arena of treatment modalities in advanced SI-NETs.

In order to move towards precision medicine, the genomic landscape of SI-NETs has been under increasing investigation over the past years in the hope of unravelling the molecular events underlying NET tumorigenesis, facilitating the identification of 
novel therapeutic targets, rational (targeted) therapy management strategies and to improve prognosis. Recently, whole-genome sequencing of primary pancreatic NETs revealed several genomic events, which characterise their pathogenesis and are associated with tumour progression. ${ }^{10}$ In general, gene expression-based subtyping has led to new classifications of multiple tumour types. In contrast, the genomic landscape of SI-NETs remains poorly elucidated and biomarkers have not yet been identified. Moreover, the genetic constitution of SI-NETs has been shown to differ compared to pancreatic NETs. ${ }^{11}$ With this review we aim to provide the clinician treating SI-NETs with an overview of the recent studies evaluating molecular characteristics of SI-NETs and their predictive and prognostic significance.

\section{Method}

A literature search was performed in PubMed in March 2019. As our main objective was to provide an up to date overview of the current literature regarding prognostic molecular factors in SI-NETs for clinicians treating patients with SI-NETs, we did not aim to perform a formal systematic review. The domain of this search consisted of adult patients with sporadic SI-NETs, the determinant of genetic or epigenetic alterations and the outcomes of prognosis, survival or progression. Synonyms of SINETs and (epi)genetic alterations with the outcome described as prognosis, survival and progression were used for the search. Search terms and syntax are described in detail in Table 1. Screening based on title and abstract was conducted by one reviewer, in case of uncertainties a second reviewer was consulted. Citation search of the included articles was performed to identify additional original studies. Inclusion criteria consisted of patient populations $>18$ years, human, full-text available in English, published between 01/01/2000 and 01/03/2019 and studies on gastroenteropancreatic NETs. Studies with a patient population with underlying genetic syndromes, no separate genome analysis for SI-NETs, using previously published results and on the taxonomy of SI-NETs were excluded. 
Table 1: Search terms and syntax

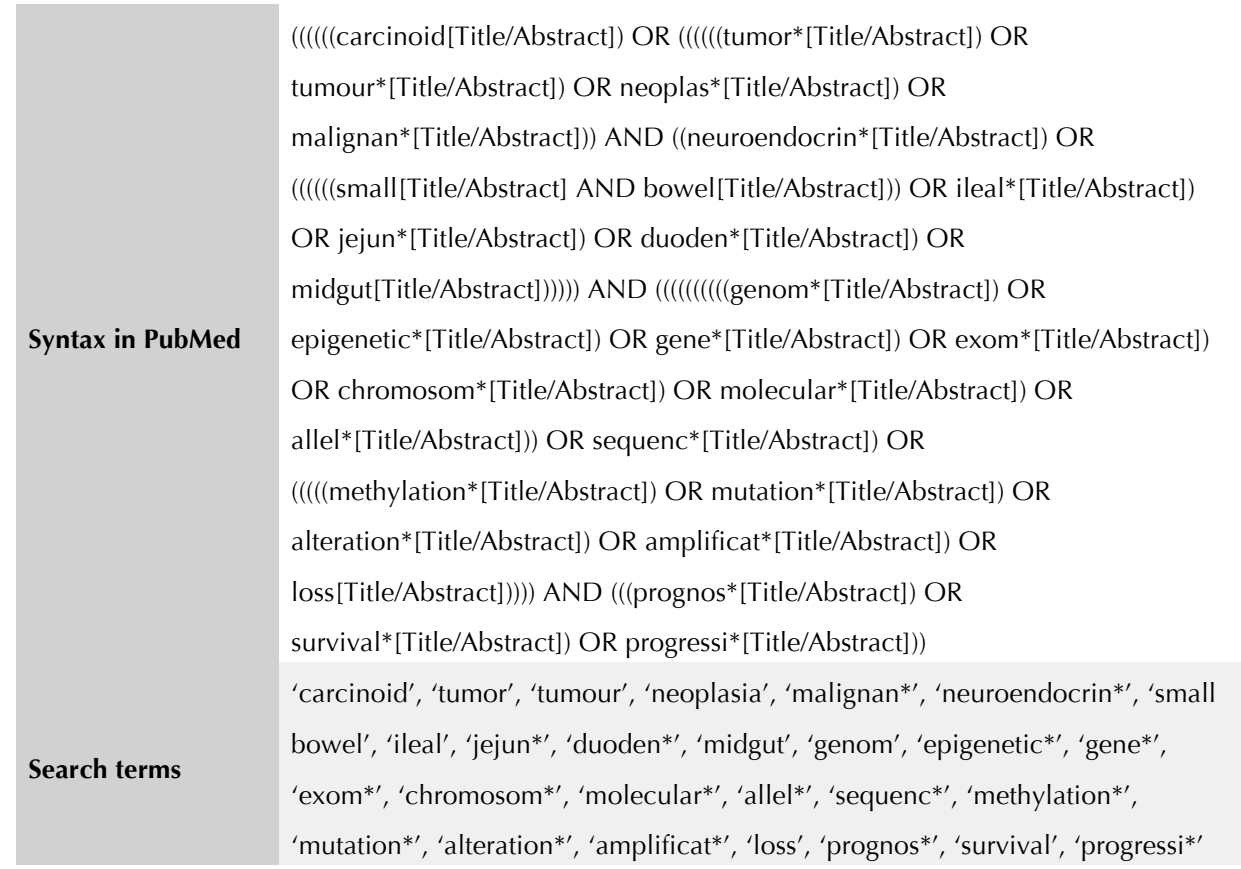

\section{Results}

The PubMed search yielded 1522 hits, of which 1461 articles were excluded after screening of title and abstract (Figure 1).

Following the full-text screening of 61 articles, 14 articles were excluded. The citation search identified 22 additional articles of which 7 were excluded. In total 55 relevant articles were found, consisting of 20 reviews and 35 original studies. The results of the selected original studies are shown in Table 2.

Our review will discuss the most relevant studies, with a special focus on the prognostic implications of the identified molecular alterations. The identified studies describe different genomic events and altered expression of several proteins, which play a key role in various molecular pathways involved in SI-NET tumorigenesis. Events that have been described in multiple studies and are discussed in this paper are shown in Figure 2. 
Part I | Chapter 3

\section{Excluded ( $\mathrm{N}=1461$ )}

Not human or children $\mathrm{N}=28$

No GEP-NETs N=1181

Genetic syndromes $N=36$

No separate analysis SI-NETs $\mathrm{N}=36$

Management of (GEP)-NETs N=180

\section{Excluded ( $\mathrm{N}=14)$}

No full tekst available $\mathrm{N}=4$

No separate analysis SI-NETs $\mathrm{N}=3$

No genome analysis $\mathrm{N}=5$

Previously published results $\mathrm{N}=1$

Taxonomy of SI-NETs N=1

\section{Title and abstract screening} $\mathrm{N}=61$

Total inclusions after full tekst screening $\mathrm{N}=55$

Reviews $\mathrm{N}=20$

Original studies $\mathrm{N}=35$

\section{Excluded ( $\mathrm{N}=7$ )}

No full tekst available $\mathrm{N}=1$

No separate analysis SI-NETs $\mathrm{N}=5$

Taxonomy of SI-NETs N=14

Figure 1: Flowchart of search and screening process in PubMed

GEP: gastroenteropancreatic; SI: small intstinal; NET: neuroendocrine tumour 

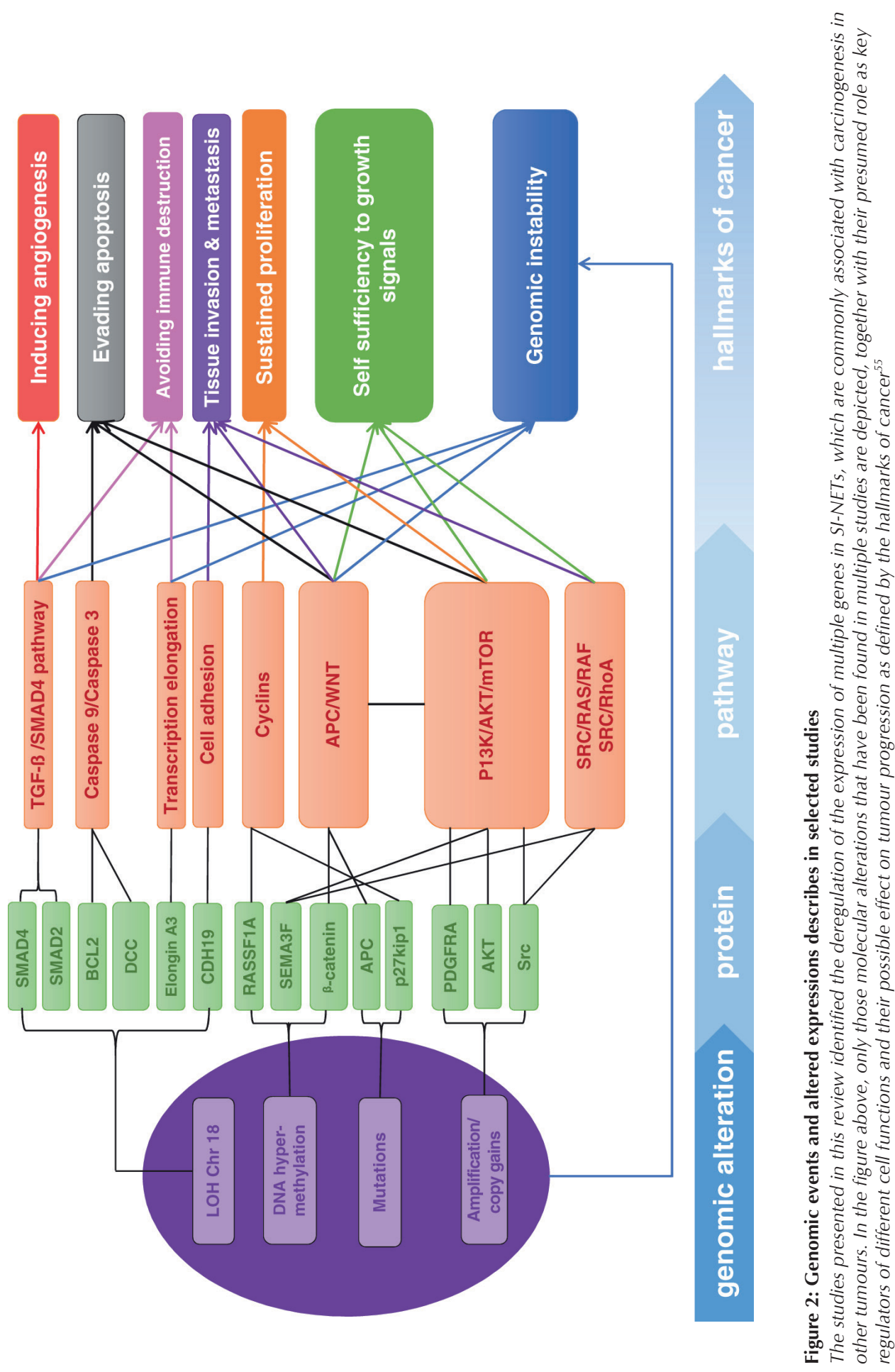
Part I | Chapter 3

- Genetics of SI-NETs

\section{Chromosomal aberrations}

From genomic profile studies, two different groups of SI-NETs can be identified, one which is characterised by loss of heterozygosity ( $\mathrm{LOH}$ ) of chromosome 18 as an early event and the other group which has no alterations of chromosome 18 and shows clustered gains on chromosomes 4, 5, 7, 14 and 20. ${ }^{11-15}$

Multiple studies reported loss of one copy of chromosome 18, with an incidence of 44-100\% in primary SI-NETs. ${ }^{11-22}$ Chromosome 18 harbours several candidate tumour suppressor genes, including DCC (deleted in colorectal cancer; involved in axon guidance), SMAD4 (mothers against decapentaplegic homolog 4; TGFB signal transduction), SMAD2 (mothers against decapentaplegic homolog 2; TGFB signal transduction) and TCEB3C (transcriptional elongation factor B polypeptide 3C; encoding Elongin A3; RNA transcription). Banck et al., who performed whole-exome sequencing (WES) on 48 well-differentiated SI-NETs, found SMAD2 and SMAD4 monoallelic deletions in 21 tumours. ${ }^{23-24}$ Edfeldt et al. $(n=43)$ identified that in the majority of SI-NETs decreased expression of Elongin A3 (77\%) was present and that the remaining TCEB3C gene was epigenetically silenced by DNA hypermethylation. ${ }^{25}$

Nieser et al. ( $n=148)$ performed the first comprehensive study to identify chromosome 18 related events at genetic, epigenetic and gene/protein expression level, which only found DCC to be affected by the monoallelic loss of chromosome $18 .^{22}$ In addition, Simbolo et al. $(n=52)$ observed copy loss of multiple genes located on chromosome 18: CDH19 (cadherin 19; cell adhesion; 46.2\%), BLC2 (B-celllymphoma; regulation of cell death; 42.3\%), DCC (42.3\%) and SMAD4 (28.8\%). ${ }^{15}$ The clinical significance of LOH of chromosome 18 has been evaluated in multiple studies, either focussing solely on LOH of chromosome 18 or as part of a molecular profile study. According to Andersson et al. $(n=43) \mathrm{LOH}$ of chromosome 18 is associated with worse overall survival. ${ }^{13}$ In contrast, Kim et al. did not find a significant correlation between loss of chromosome 18 and survival. ${ }^{18}$ Contrarily, Yao et al. $(n=89)$ found that SI-NETs with low generalised chromosomal instability (CIN) (which consisted of a cluster with LOH of chromosome 18) displayed significantly longer median PFS than those with a high CIN (which consisted of 3 clusters with different combinations of gains of chromosome 4, 5, 7, 14, 20 and 1 
cluster with copy number gains across most chromosomes). PFS in patients with a low CIN ( $n=55)$ was 18.6 vs 9.2 months in high $\mathrm{CIN}(\mathrm{n}=38)(\mathrm{HR} ; 0.41 ; 95 \% \mathrm{Cl}$ $0.24-0.73 ; \mathrm{P}=0.0021)^{11}$

As described by the clusters of Yao et al., recurrent gains of chromosome 4, 5, 7, 14 and 20 are common in SI-NETs. ${ }^{11-15,18,19,21}$ In two studies by Andersson et al., gain of chromosome 14 was seen in 6 of 32 well-differentiated SI-NETs and was associated with higher tumour grade and shorter survival ( $\mathrm{HR}$ 8.39; 95\% Cl 3.04-23.11)..$^{13,21}$ However, Cunningham et al. $(n=45)$ and Simbolo et al. $(n=52)$ could not corroborate these findings. ${ }^{15,19}$ Hashemi et al. $(n=30)$ studied copy number alterations (CNAs; gains and losses of areas of the chromosome) and reported an association between gain of 20pter-p11.21 and worse survival, which was also not confirmed by the findings of Simbolo et al. ${ }^{14,15}$ Generalised chromosomal instability seems to be a common feature of SI-NETs. This phenomenon could possibly be a reflection of diverse underlying defects in chromosomal maintenance that drive SINET development. ${ }^{11}$

\section{Mutational status}

Banck et al. analysed 48 primary SI-NETs, predominantly grade 1 , by massively parallel exome sequencing and detected a low mutation rate in the SI-NET genomes with an average of 0.1 somatic single-nucleotide variants (SNVs) per 106 nucleotides in the exome, suggesting that SI-NETs are mutationally quiet tumours. ${ }^{23}$ No recurrent mutations in the 215 sequenced target genes were found. In the studied SI-NETS, 197 protein-altering $S N V s$ were identified, affecting a multitude of cancer genes including FGFR2, MEN1, HOOK3, EZH2, MLF1, CARD11, VHL, NONO, FANCD2, SMAD1 and BRAF. In 29\% of SI-NETs, there were genetic alterations in the P13K/AKT/mTOR pathway and mutually exclusive amplification of AKT1 or AKT2 were common. Amplifications were also observed at the PDFDR (platelet-derived growth factor receptor alpha) locus in $20.8 \% .^{23}$ In a recent study by Simbolo et al. frequent copy gains were detected in AKT1 (30.8\%) and PDGFRA (platelet-derived growth factor receptor alpha: $28.8 \%$ ) as well. Furthermore, gains were present at the FOS gene (transcription factor subunit; 36.5\%), KIT (involved in cell proliferation, survival, migration and differentiation; 28.8\%) and KDR (kinase insert domain receptor, involved in VEGF signalling; $28.8 \%$ ) genes. ${ }^{15}$ Higher mutation rates in primary SINETs were associated with increased likelihood of recurrent liver metastases $(\mathrm{P}<0.04) .{ }^{23}$ In a study by Francis et al. $(\mathrm{n}=180)$ including 48 cases from Banck et al., 
heterozygous frame shift mutations of the cyclin-dependent kinase inhibitor $1 \mathrm{~B}$ gene (CDKN1B) in 14 of 180 SI-NETs $\left(8 \%\right.$; 95\% Cl 4.7-12.7\%) were observed. ${ }^{24}$ CDKN1B is located on chromosome 12 and encodes the protein p27Kip1, a cyclin-dependent kinase inhibitor $(\mathrm{CKI})$, whose main function is to control the progression from $\mathrm{G} 1$ to $\mathrm{S}$ phase in the cell cycle. The reported mutations in this putative tumour suppressor gene in SI-NETs are loss-of-function truncating mutations throughout the gene; no hotspot has been identified.

A further study by Crona $(\mathrm{n}=200)$, confirmed the presence of CDKN1B mutations in 17 of 200 SI-NETs (8.5\%) $(95 \% \mathrm{Cl} 4.6-12.4 \%) .{ }^{26}$ Mutational status did not appear to correlate with protein expression of p27Kip1 and no immediate detectable impact on clinical phenotype and survival was found. ${ }^{26}$ Similarly, Shi et al. observed CDKN1B mutations in $10.9 \%$ of 55 SI-NETs and found no association between CDKN1B mutation, p27kip1 expression and survival. ${ }^{27}$ Only a trend towards shorter survival of patients with tumours exhibiting low expression of p27kip1 (multivariate HR, 2.04; 95\% Cl 1.06-3.93; $\mathrm{P}=0.03$ ) was observed. Other studies found CDKN1B mutations in $4.5-9.6 \%$ of SI-NETs. ${ }^{11,15,28,29}$ Furthermore, Yao et al., using whole-exome and targeted panel sequencing on 89 SI-NETs from the RADIANT trials, found recurring mutations in BCOR (BCL-6-interacting corepressor) in 5.6\%. ${ }^{11}$ BCOR has interactions with histone deacetylases which are involved in the regulation of gene expression through DNA methylation. ${ }^{11}$ Another recent study, by Simbolo et al., performed targeted sequencing on 52 primary SI-NETs of which $34.6 \%$ showed somatic mutations. ${ }^{15}$ APC (adenomatous polyposis coli; Wnt signalling pathway regulator) and CDKN2C (CKI 2C; cell growth regulator which controls cell cycle G1 progression) were found to be recurrently mutated in $7.7 \%$. In addition, mutations were found in known oncogenes such as BRAF (involved in MAPK/ERK signalling pathway), KRAS (involved in RAS/MAPK signalling pathway), PIK3CA (phosphatidylinositol-4,5-bisphosphate 3-kinase catalytic subunit alpha; involved in the P13K/AKT/mTOR pathway) and TP53 (tumour suppressor gene; regulator of cell proliferation and apoptosis) in 3.8\%. ${ }^{15}$ Previously, Bottarelli et al. $(\mathrm{n}=30)$ described APC gene alterations in $23 \%$ of ileal NETs. ${ }^{30}$ A copy gain of the SRC gene (protooncogene; involved in cell signalling), which was present in $25 \%$ of SI-NETs, was associated with poorer prognosis $(P=0.047) .{ }^{15}$ The SRC gene (cell signalling, cell cycle control and cell adhesion) was also the most commonly amplified oncogene $(23 \%)$ in the study by Banck et al. ${ }^{23}$ Copy gains of the SRC gene could potentially be a novel prognostic biomarker, especially as whole-genome sequencing becomes more widely adopted into clinical practice of SI-NETs. 
Molecular alterations in primary tumours vs. metastases

Molecular differences between primary tumours vs. metastases can provide insight in the process of tumour progression. Cunningham et al. observed increased gains of chromosome 7 in metastases (30 mesenterial and 4 hepatic) vs. primary SI-NETs $(16 \%$ vs. $0 \%) .{ }^{19}$ Correspondingly, Hashemi et al. reported frequent gain of $7 q 22.3-$ qter in metastases (12 regional and 7 distant; $\mathrm{P}=0.016$ ) compared to primary tumours (14). Loss of 16q12.2qter was more common in distant metastases vs. primary tumours $(\mathrm{P}=0.003) .{ }^{14}$ Karpathakis found $\mathrm{LOH}$ of chromosome 18 in $79 \%$ in liver metastases. ${ }^{31}$ In the same study, amplification of chromosome 20 was found in $42 \%$, deletion of chromosome 19 in $34 \%$ and gain of chromosome $17 \mathrm{q}$ in $21 \%$ of liver metastases. ${ }^{31}$ Furthermore, at mRNA level, analysis of differentially expressed genes between liver metastases and primary tumours identified significant enrichment of multiple cancer-related pathways overexpressed in liver metastasis, for example P13K signalling events, ERBB1 downstream signalling, PDGFRB signalling and the mTOR pathway (adjusted $\mathrm{P}<0.001$ ). ${ }^{31}$ Keck et al. demonstrated by RNA sequencing that liver metastases show underexpression of PMP22 (peripheral myelin protein 22; integral membrane protein involved in demyelinating disease and apoptosis) compared to the corresponding primary tumour $(<0.001)(32)$. Fotouhi et al. $(n=33)$ found different expression levels of CXCL14 (chemokine CXC motif ligand 14 ; involved in cytokine activity and angiogenesis) mRNA in metastases compared to primary tumours $(\mathrm{P}=0.0016)$, which correlated with methylation status of the respective genes. ${ }^{33}$ Furthermore, increased expression was found for mRNA encoding beta-catenin (involved in Wnt signalling pathway) in metastases compared with primary tumours $(\mathrm{P}=0.041)$; for mRNA encoding P16 (regulates entry into S phase) in distant metastasis compared to primary tumours and regional metastases $(\mathrm{P}=0.015)$ and for mRNA encoding RASSF1A (involved in cell cycle regulation, apoptosis and migration), in regional metastases compared to primary tumours and distant metastases $(P=0.008)$. Low mRNA expression of RASSF1 A and P16 were each associated with short survival $\left(P=0.045\right.$ and $P=0.011$, respectively). ${ }^{33}$ Using gene expression arrays, Edfeldt et al. were able to identify differentially expressed mRNA in SI-NET metastases compared to primary tumours which resulted in the identification of three different gene expression clusters. However, these clusters did not correlate with tumour progression. ${ }^{34}$ To conclude, dissemination of SI-NETs is associated with genomic events; yet the way in which these events contribute to tumour progression remains unclear. 
Prognostic stratification based on LOH of chromosome 18, CDKN1B mutations, CpG island methylator phenotype and copy number variations

Karpathakis et al. identified different prognostic subgroups using hierarchical clustering. In a sophisticated large-scale integrated genomic analysis, including DNA methylation, gene expression and copy number variance (CNV) of 97 SI-NETs from a cohort of 85 patients they identified three molecular subtypes of SI-NETs using an integrated genome analysis. ${ }^{29}$ The largest subgroup (55\%) was defined by chromosome $18 \mathrm{LOH}$ and is associated with the presence of CDKN1B mutations, and $\mathrm{CpG}$ island methylator phenotype (CIMP) negativity. The CpG island methylator phenotype refers to the DNA hypermethylation of promoter-associated CpG islands of tumour suppressor and DNA repair genes, which leads to transcriptional silencing of these genes. These patients had the most favourable PFS (not reached at 10 year follow-up) after resection and a median age of 67 years at diagnosis. A second subgroup (18\%) was characterised by the absence of arm-level CNVs (copy number variations that span the chromosomal arm) and a high degree of CIMP positivity. This group had an intermediate PFS (56 months) and a younger median age at diagnosis (60 years). The third subgroup consisted of $26 \%$ of SI-NETs and was characterised by multiple CNVs; these patients had a significantly poorer PFS (21 months) and were youngest at onset (54 years), suggesting a more aggressive clinical phenotype. In accordance with Karpathakis et al., Yao et al. $(n=89)$, identified similar prognostic groups regarding LOH of chromosome 18 and alterations in chromosome 4, 5 and 20. ${ }^{11}$ However, Simbolo et al. classified their cohort $(n=52)$ into the three molecular groups of Karpathakis et al. and did not observe any statistically significant correlation with prognosis $(\mathrm{P}=0.73) .{ }^{15}$ These results of Simbolo et al. could be due to the relatively small cohort in comparison to the cohort of Karpathakis et al. and do not necessarily weaken the findings of Karpathakis and Yao et al. Based on the findings of Karpathakis et al. and considering the relatively low frequency of somatic mutations in SI-NETs, it seems unlikely that mutations of the CDKN1B gene or LOH of chromosome 18 alone are driving the SI-NET tumorigenesis and suggests a greater role for epigenetic dysregulation. ${ }^{11,15,20,25,29,35}$

\section{- Germline mutations in SI-NETs}

A germline mutation is defined as a mutation that occurs in reproductive cells and therefore is incorporated in every cell of the offspring. A study by Dumanski et al. $(\mathrm{n}=239)$ sequenced germline DNA from 24 patients from 15 families with a history 
of SI-NETs and from 215 patients with a sporadic SI-NET. ${ }^{36}$ A mono-allelic mutation causing an amino-acid substitution p. (Gly396Asp) in MUTYH was found to be significantly enriched in both patients affected with familial SI-NETs and in sporadic SI-NETs, compared to controls (minor allele frequencies 0.013 and 0.03, respectively) with an odds ratio of 5.09 (95\% $\mathrm{Cl} 1.56-14.74 ; \mathrm{P}=0.0038)$. MUTYH encodes a DNA glycosylase, involved in repair of oxidative DNA damage in order to prevent mutation accumulation leading to tumorigenesis. Biallelic germline MUTYH mutations lead to multiple colorectal adenomas and carcinomas, referred to as MUTYH-associated polyposis (MAP), a recessive hereditary colorectal polyposis syndrome. Interestingly, MUTYH germline mutations were also found in pancreatic NETs. ${ }^{10,37}$ By defective DNA repair, carriers with MUTYH mutations thus seem to have a predisposition to develop NETs of the pancreas or small intestine.

\section{- Epigenetics in SI-NETs}

Epigenetic modification can be defined as a change in gene expression without alterations to the gene's DNA sequence itself. ${ }^{38}$ Since SI-NETs appear to have relatively few somatic mutations, epigenetic dysregulation could play an important role in the tumorigenesis of SI-NETs and may have important clinical implications. ${ }^{23,24,35}$ Epigenetic changes include DNA methylation, histone modifications and the actions of miRNA. Hypermethylation and hypomethylation and histone modifications modify gene expression, whereas miRNAs, small singlestranded RNA molecules, regulate gene expression post-transcriptionally.

These processes can be pharmacologically modified by targeting enzymes involved in DNA methylation and histone modifications, and by miRNA inhibitors thereby representing an appealing target for therapy. ${ }^{35,39}$

In comparison with genetic mutations, epigenetic alterations are significantly more common and recurrent in SI-NETs. Our search yielded studies ranging from 8 to 97 patients that showed epigenetic alterations in SI-NETs for example DNA methylation changes in $65-82 \%$ of SI-NETs and multiple miRNA deregulations. ${ }^{29,40-42}$ Several studies reported differences in methylation and miRNA patterns between primary tumours and metastases, suggesting a possible role in tumour development or progression. A recurrent event is the epigenetic silencing of RASSFIA (RASassociation domain family 1 , isoform A gene; tumour suppressor gene inducing cell cycle arrest) expression by hypermethylation of its promotor. This event was observed by Choi et al., Zhang et al. and Fotouhi et al. and was more prominent in 
Part I | Chapter 3

Table 2: Overview of studies

\begin{tabular}{|c|c|c|c|c|}
\hline Study & $\begin{array}{l}\text { No. of } \\
\text { patients }\end{array}$ & Domain & Analysis technique & Remarks \\
\hline Löllgen 2001 & 8 & $\begin{array}{l}\text { Metastatic midgut } \\
\text { NETs ( } 6 \text { ileal, } 1 \\
\text { ileocecal valve, } 1 \\
\text { ascending colon) }\end{array}$ & $\begin{array}{l}\text { Genome-wide } \mathrm{LOH}^{*} \text { screening } \\
\text { with microsatellite markers }\end{array}$ & $\begin{array}{l}\text { Analysis } \\
\text { included } 1 \\
\text { colon NET }\end{array}$ \\
\hline Wang 2005 & 47 & Ileal NETs $(n=16)$ & $\begin{array}{l}\text { Microsatellite markers, PCR } \\
\text { amplification, } \\
\text { sequencing } B R A F \text { gene }\end{array}$ & - \\
\hline Zhang 2006 & 33 & $\begin{array}{l}\text { SI-NETs with } \\
\text { matched primary and } \\
\text { metastatic tumours }\end{array}$ & $\begin{array}{l}\text { Methylation-specific PCR, } \\
\text { Western blot and } \\
\text { immunochemistry }\end{array}$ & - \\
\hline Kim 2007 & 29 & $\begin{array}{l}\text { Well-differentiated } \\
\text { ileal NETs }(n=15)\end{array}$ & $\begin{array}{l}\text { Genome-wide high-density } \\
\text { single-nucleotide polymorphism } \\
\text { array analysis }\end{array}$ & - \\
\hline
\end{tabular}

Choi $2007 \quad 35 \quad$ Ileal NETs $(n=15) \quad$ Pyrosequencing

$\begin{array}{lll}\text { Kulke 2008 } & 18 \quad \begin{array}{l}\text { Primary and } \\ \text { metastatic SI-NETs } \\ (n=24)\end{array} & \begin{array}{l}\text { High resolution arrays of single- } \\ \text { nucleotide polymorphisms }\end{array}\end{array}$

$\begin{array}{lll}\begin{array}{l}\text { Andersson } \\ \mathbf{2 0 0 9}\end{array} & 43 \quad \text { Ileal NETs } & \begin{array}{l}\text { High-resolution array based on } \\ \text { comparative genomic } \\ \text { hybridisation }\end{array}\end{array}$

$\begin{array}{llll} & & \begin{array}{l}\text { Sporadic and familial } \\ \text { ileal NETs (37 }\end{array} \\ \text { Cunningham } & 45 \quad \begin{array}{l}\text { sporadic and } 8 \\ \text { familial; } 61 \text { tumour } \\ \text { samples) }\end{array} & \begin{array}{l}\text { High-resolution genomic and } \\ \text { gene expression profiling }\end{array}\end{array}$

$\begin{array}{lll}\text { Ruebel } 2010 & 8 & \begin{array}{l}\text { Primary and } \\ \text { metastatic ileal NETs }\end{array}\end{array}$

RT-PCR, miRNA expression assay, Northern blotting, in situ hybridisation
*MPTR $<1$

(upregulated in primary tumours) *MPTR > 1 (downregulated in primary tumours) 
Deletions on Chr* 18 in $88 \%$ of midgut NETs

Allelic loss of both arms of chromosome 18 in $69 \%$; No BRAF mutations were identified

Methylation of RASSF1A and CTNNB1 promoters more frequent in metastatic vs primary tumours $(P=0.013$ and $P=0.004$, respectively)

Loss of Chr 18 in $67 \%$, loss of Chr 21 or 21 q in $13 \%$

No correlation between loss of Chr 18 and 21 with survival

No survival differences were observed based on LINE-1 methylation levels
Hypomethylation of LINE-1 was greater in ileal NETs than in non-ileal and pNETs $(P=0,047)$, and tumours with lymph node metastasis $(P=0.02)$, Chr 18 loss $(P=0.001)$ and RAS-association domain family $\mathrm{I}$, isoform A gene methylation $(P=0.02)$.

Loss of Chr 18 in 61\%, Chr 9 in 33\% and Chr 16 in $22 \%$. Gains on Chr 4 (33\%) and Chr 5,7,20 and $14 q$ $(17 \%)$

Loss of Chr 18 in 74\%. Other frequent copy number alterations were gain of Chr 4 (30\%), 5 (28\%), 14 (23\%) and $20(33 \%)$, and loss of $11 q 22.1-q 22.2,11 q 22.3-$ $\mathrm{q} 23.1$ and $11 \mathrm{q} 23.3(21 \%)$ and loss of $16 \mathrm{q} 12.2-\mathrm{q} 22.1$ and 16q23.2-qter (16\%)
Gain of Chr 14 was a strong predictor of poor survival $(P<0.001$; HR 8.39 (95\% $\mathrm{Cl}$ : 3.04-23.11)). Loss of $3 \mathrm{p} 13$ resulted in a reduced risk of death $(P=0.028 ; \mathrm{HR}$ 0.14 (95\% Cl: 0.02-0.8)). Significant correlation between gain of Chr 7, 14 and 20 , and loss of 18 and overall survival $(P<0.05)$
Chr 18 aberrations in both sporadic and familial ileal NETs (100 vs 38\%) Frequent gain of Chr 7 in metastasis vs primary tumour (16 vs $0 \%$ )
No difference in overall survival in patients with or without a gain of Chr 14

Downregulation of miRNA-133a (ratio $\left.0.27^{*}\right),-145$

(ratio $\left.0.33^{*}\right),-146\left(\right.$ ratio $\left.0.36^{*}\right),-222$ (ratio $0.41^{*}$ ) and

-10 b (ratio $0.44 *$ ) in $100 \%$ of primary vs matching metastasis, upregulation of miRNA-183 (ratio 1.99*), -488 (ratio $1.56^{*}$ ), and $-19 a+b$ (ratio $1.31^{*}$ ) in $75 \%$ metastatic ileal NETs compared to primary tumours 


\begin{tabular}{|c|c|c|c|c|}
\hline $\begin{array}{l}\text { (continuation) } \\
\text { Study }\end{array}$ & $\begin{array}{l}\text { No. of } \\
\text { patients }\end{array}$ & Domain & Analysis technique & Remarks \\
\hline Walsh 2011 & $\begin{array}{c}239 \\
\text { cases, } \\
110 \\
\text { controls }\end{array}$ & Ileal NETs & $\begin{array}{l}\text { Genome-wide association study } \\
\text { single-nucleotide polymorphism } \\
\text { genotyping }\end{array}$ & - \\
\hline Edfeldt 2011 & 19 & $\begin{array}{l}\text { SI-NETs }(n=18), \\
\text { lymph node } \\
\text { metastases }(n=17) \text {, } \\
\text { liver metastases } \\
(n=7)\end{array}$ & Gene expression arrays, qPCR & - \\
\hline Stricker 2012 & 58 & SI-NETs $(n=17)$ & Pyrosequencing & - \\
\hline Banck 2013 & 48 & SI-NETs & Exome sequencing & - \\
\hline Francis 2013 & $\begin{array}{l}180, \\
(48 \\
\text { from } \\
\text { Banck } \\
2013)\end{array}$ & SI-NETs & Exome and genome sequencing & - \\
\hline Li 2013 & 24 & $\begin{array}{l}\text { SI-NETs ( } 5 \text { primary } \\
\text { tumours, } 5 \\
\text { mesentery } \\
\text { metastasis, } 5 \text { liver } \\
\text { metastasis) }\end{array}$ & $\begin{array}{l}\text { Affymetrix Genechip miRNA } \\
\text { array, qRT-PCR, Northern blot } \\
\text { Analysis }\end{array}$ & - \\
\hline
\end{tabular}

$\begin{array}{llll}\text { Hashemi } 2013 & 20 & \begin{array}{l}\text { SI-NETs }(n=18) \text { and } \\ \text { metastases }(n=12)\end{array} & \begin{array}{l}\text { Comparative genome } \\ \text { hybridisation, qPCR }\end{array}\end{array}$

Bottarelli $2013 \quad 30 \quad$ Ileal NETs

DNA fragment analysis and sequencing of the mutation cluster region of the $A P C$ gene 
No single-nucleotide variants significantly associated with ileal NETs, rs2208059 in KIF16B had a trend towards statistical significance; $14 / 226$ cases $(6.19 \%)$ and $2 / 97$ controls $(2.06 \%)$ heterozygous copy number deletions at $18 q 22.1$

Three clusters of gene expression profiles were identified distinguishing primary tumours (11/18) from lymph node metastases (5/17) and a third group consisting of liver metastases (7/7), lymph node metastases (12/17) and primary tumours $(7 / 8)$. The different profiles suggest changes in the development from primary tumour to No association was found between group 1 and group 3 for indolent or progressive disease course $(P=0.15)$ metastases

LINE1 hypomethylation was detected in $82 \%$ of SI-NETs

0.1 SNVs* per 106nucleotides. No recurrent mutations in cancer genes. 197 protein-altering SNVs affected multiple cancer genes, including FGFR2, MEN1, HOOK3, EZH2, MLF1, CARD11, VHL, NONO and SMAD1. Mutually exclusive amplification of AKT1 or AKT2 was the most common event in 16 patients with alteration of $\mathrm{P} 13 \mathrm{~K} / \mathrm{Akt} / \mathrm{mTOR}$ signalling

Frameshift mutations of CDKN1B in $8 \%$ SI-NETs $(8 \%$; 95\% Cl 4.7-12.7), hemizygous deletions encompassing CDKN1B in $14 \%$

miRNA-96 $(P<0.01$ compared to mesentery metastasis

$(\mathrm{MM})$ and $P<0.001$ liver metastases (LM)), -182

$(\mathrm{MM} P<0.05, \mathrm{LM} P<0.001),-183(\mathrm{MM} P<0.001$,

LM $P<0.01)$, -196a (MM $P<0.001$, LM $P<0.001)$ were

upregulated during tumor progressio; miRNA-31

$(\mathrm{MM} \mathrm{P}<0.05, \mathrm{LM}<0.05),-129-5 \mathrm{p}(\mathrm{MM} \mathrm{P}<0.01$,

LM P $<0.001),-133 a(M M P<0.05, L M<0.05)$ and

$-215(\mathrm{MM} \mathrm{P}<0.05, \mathrm{LM} \mathrm{P}<0.05)$ were downregulated

Loss of chromosome 18 in 70\%. Copy number losses on chromosome 11 (23\%), 16 (20\%), and 9 (20\%), with regions of recurrent copy number loss identified in 11q23.1-qter, 16q12.2-qter, 9pter-p13.2 and 9p13.111.2. Gains detected in chromosomes 14 (43\%), 20 (37\%), $4(27 \%)$, and 5 (23\%) with recurrent regions of copy number gain in $14 q 11.2,14 q 32.2-32.31,20$ pterp11.21, 20q11.1-11.21, 20q12-qter, 4 and 5.

Gain in 20pter-p11.21 was associated with short survival $(P=0.013)$. No other significant associations were observed between recurrent copy number alterations and survival

Differences between primary tumours and metastases; loss of $16 \mathrm{q}(P=0.003)$ loss and gain of Chr $7(P=0.016)$

APC gene mutations in $23 \%$, of which missense $(57 \%)$ and nonsense/frameshift (14\%) mutations 


\begin{tabular}{|c|c|c|c|c|}
\hline $\begin{array}{l}\text { (continuation) } \\
\text { Study }\end{array}$ & $\begin{array}{l}\text { No. of } \\
\text { patients }\end{array}$ & Domain & Analysis technique & Remarks \\
\hline Edfeldt 2013 & 43 & SI-NETs & $\begin{array}{l}\text { Gene copy number } \\
\text { determination by PCR, real time } \\
\text { quantitative RT-PCR, RNA } \\
\text { interference, CpG methylation } \\
\text { pyrosequencing }\end{array}$ & - \\
\hline Fotouhi 2014 & 33 & SI-NETs $(n=44)$ & $\begin{array}{l}\text { Pyrosequencing, ELISA-based } \\
\text { quantification of global DNA } \\
\text { methylation, qRT-PCR }\end{array}$ & - \\
\hline Verdugo 2014 & 20 & $\begin{array}{l}\text { Matched primary SI- } \\
\text { NETs }(n=10) \text { and } \\
\text { their mesenteric } \\
\text { lymph node } \\
\text { metastases }(n=10)\end{array}$ & $\begin{array}{l}\text { Human methylation } 27 \\
\text { BeadChip array profiling }\end{array}$ & - \\
\hline Norlen 2014 & 15 & $\begin{array}{l}\text { Peritoneal } \\
\text { carcinomatosis of SI- } \\
\text { NETs }(n=8) \text { and } \\
\text { controls }(n=7)\end{array}$ & $\begin{array}{l}\text { Single-nucleotide polymorphism } \\
\text { array }\end{array}$ & - \\
\hline
\end{tabular}

$\begin{array}{cll}\text { Crona } 2015 & \text { Automated Sanger sequencing of } \\ \text { SI-NETs }(n=362) \quad & \begin{array}{l}\text { the CDKN1B gene, } \\ \text { immunohistochemistry }\end{array}\end{array}$

$\begin{array}{llll}\text { Maxwell } 2015 & 90 & \text { SI-NETs } & \begin{array}{l}\text { Exome sequencing and CNV } \\ \text { analysis by quantitative PCR }\end{array} \\ \begin{array}{l}\text { Delgado } \\ \text { Verdugo 2015 }\end{array} & 7 & \text { SI-NETs } & \begin{array}{l}\text { Whole-exome capture, NGS*, } \\ \text { high resolution SNP array, copy } \\ \text { number variation analysis }\end{array}\end{array}$


One copy deletion in $89 \%$ SI-NETs with reduced Elongin A3 expression in $77 \%$.
Methylation was seen in WIF1(methylation index (MI) 50\%, (16-92\%)), RASSFA1(MI 16\% (1$69 \%))$, CTNNB1 (MI 13\% (4-34\%)), CXCL14(MI 14\% (3-39\%)), NKX2-3 (MI 10\% (2-28\%)), P16(CDKN2A) (MI 4\% (1-33\%)), LAMA1(MI 10\% (4-24\%), and CDH1 (MI 8\% (3-22\%). APC (MI 3\% (2$8 \%)$ ), CDH3(MI 6\% (3-12\%)), HIC1 (MI 5\% (1$12 \%)$ ), P14(CDKN2A) (MI 5\% (2-17\%)), SMAD2(MI 4\% $(1-8 \%)$ ) and SMAD4 (MI 3\% (1-6\%) had low levels of methylation. WIF1 methylation was significantly increased $(P=0.001)$ and WIF1 expression was reduced in SI-NETs vs normal references $(P=0.003)$. WIF1, NKX2-3 and CXCL14 expression was reduced in metastases vs primary tumours $(P<0.02)$. Global methylation of LINE1 was reduced in tumours vs normal references (65 vs $75 \%$ ), and was associated with loss of Chr18p and $18 \mathrm{q}(P=0.022, P=0.003$, respectively)

RUNX3, TP73 and CHFR were highly methylated $(\beta$ value $\geq 0.9$ ). At Chr 18q21-qter ( $\beta$ value $>0.7)$, SETBP1, ELAC1, MBD1, MAPK4, and TCEB3C were methylated including several members of the Serpin peptidase inhibitor family (SERPINB3, SERPINB5).
Low expression of RASSF1A and P16 were associated with poor survival $(P=0.045$ and $P=0.011$, respectively). Genespecific promoter methylation or global methylation did not influence survival

Two groups were identified, group A with a greater proportion of patients with PC (86\%) than group B (25\%), with $\mathrm{LOH}$ of the entire or major part of chromosome 18 in group A (75\%) compared to limited $\mathrm{LOH}(75 \%)$ or no $\mathrm{LOH}(25 \%)$ in group $\mathrm{B}$

Mutations of $C D K N 1 B$ in $8.5 \%$. Inter- and intratumour heterogeneity at the CDKN1B locus was present (33 and $11 \%$ respectively). Expression of p27 did not correlate with CDKN1Bmutation status. No differences in clinical characteristics between $C D K N 1 B$ mutated and $C D K N 1 B$ wild-type tumour carriers were found
SI-NETs with a higher methylation index had a more aggressive phenotype

CDKN1B frameshift mutations in $3.5 \%$ of SI-NETs $(95 \%$ $\mathrm{Cl}$ : 1.1-9.8\%), 1 patient had a hemizygous deletion of CDKN1Band 2 patients duplications $(3.4 \%$; $95 \% \mathrm{Cl}$ $0.41-7.2 \%$ ). Mutations of CDKN1B occurred in $6.9 \%$

Loss of Chr18 in $71 \%$ of SI-NETs. No tumour-specific somatic mutation was identified

No correlation was found between survival and CDKN1Bmutation status (HR $0.76 ; 95 \%$ Cl $0.36-1.57$ ) 


\begin{tabular}{|c|c|c|c|c|}
\hline $\begin{array}{l}\text { (continuation) } \\
\text { Study }\end{array}$ & $\begin{array}{c}\text { No. of } \\
\text { patients }\end{array}$ & Domain & Analysis technique & Remarks \\
\hline
\end{tabular}

Bollard $2015 \quad 38 \quad$ Ileal NETs

Karpathakis

2015

$97 \quad$ SI-NETs
Immunochemistry, methylation-

specific PCR

\author{
Whole-genome or \\ targeted CDKN1Bsequencing, \\ Human methylation 450 \\ BeadChip array profiling, \\ methylated \\ DNAimmunoprecipitation \\ sequencing, CNV analysis, \\ whole-genome DASL* \\ expression array profiling
}

Primary SI-NETs $(n=28)$, adjacent normal small bowel $(n=14)$, matched lymph node

Miller 2016 90 metastases $(n=24)$, normal lymph node metastases $(n=7)$, normal liver $(n=2)$ and liver metastasis $(n=15)$
NanoString miRNA profiling, qRT-PCR, luciferase reporter assays and immunoblotting
Well-differentiated distal ileal NETs

Genome-wide sequencing

Analysis included 1 ileal NEC
Dumanski 2017
Sporadic (215) and familial (24) SI-NETs compared to three control cohorts with 35,688 subjects
NGS* of exome or wholegenome DNA
Human methylation 450

BeadChip array profiling, methylated DNA immunoprecipitation sequencing, whole-genome DASL* expression array profiling 
SEMA3Fexpression was lost in 96\% ileal NETs and all their metastases. SEMA3F loss of expression was associated with promoter gene methylation (no Pvalue provided)

Subgroup 1: chromosome $18 \mathrm{LOH}, C D K N 1 B$ mutations, CIMP*, negativity; Subgroup 2: absence of arm-level CNVs, CIMP positivit; Subgroup 3: multiple CNVs; Epimutations were found at a recurrence rate up to $85 \%$, and 21 epigenetically dysregulated genes were identified,

3 subgroups of SI-NETs with different PFS* (not reached at 10 years vs 56 months vs 21 months; $P=0.04$ ) including CDX1(86\%), CELSR(84\%), FBP (84\%), and GIPR (84\%).

miR-204-5p $(P=2.44 \times 10-67)$, miR-7-5p

$(P=2.57 \times 10-144)$ and miR-375 $(P=6.30 \times 10-67)$

were upregulated and miR-1 $(P=0.0004)$ and MiR-143$3(P=8.11 \times 10-9)$ were downregulated in lymph node and liver metastases vs primary tumours

Loss of chromosome 18 in $65 \%$ and gains of chromosome $4,5,7,14$ and 20 in $51 \%$. Loss of $C D K N 1 B$ in $8 \% .3$ subgroups were identified. The prostaglandin E receptor 2 (PTCER2) is the most activated in tumours of higher grade $(P=4.4 \times 10-10)$, whereas Forkhead box M1 (FOXM1) was the most activated regulator in tumours with gain of chromosome $14(P=2.5 \times 10-4)$
The largest subgroup $(n=17)$ was characterised by longer survival $(P<0.05)$ and higher expression of neuroendocrine markers, including SSTR2. Tumours with higher grade $(\mathrm{G} 2 / 3)$ or gain of chromosome 14 were associated with shorter patient survival $(P<0.05)$ and increased expression of cell cyclepromoting genes

A mutation in the MUTYH gene was significantly enriched in SI-NETs (both sporadic and familial) compared to controls (OR 5.09; 95\% 1.5614.74; $P=0.0038$

SI-NET liver metastases show Chr18 LOH in $79 \%$. Amplification of Chr20 (42\%), deletion of Chr19 (34\%) and gain of $17 q(21 \%)$ in liver metastasis. In liver metastasis enrichment of multiple cancer-related pathways was seen; P13Ksignalling events, ERBB 1 downstream signalling, $P D G F R B$ signalling pathway and $m T O R$ pathway (adjusted $P<0.001$ ). Using a previously defined panel of 21 epimutated genes, a trend of progressive dysregulation in liver metastasis compared to primary SI-NETs was observed 


\begin{tabular}{|c|c|c|c|c|}
\hline $\begin{array}{l}\text { (continuation) } \\
\text { Study }\end{array}$ & $\begin{array}{c}\text { No. of } \\
\text { patients }\end{array}$ & Domain & Analysis technique & Remarks \\
\hline
\end{tabular}

Shi 2017 SI-NETs $(n=55) \quad$ Immunochemistry, CDKN1B sequencing -

$\begin{array}{lll}\text { Nieser } 2017 & \text { qRT-PCR, Western blot, } \\ \text { imfl-NETs } & \begin{array}{l}\text { immunochemistry, NGS*, SNP array } \\ \text { analysis, miRNA analysis by qRT-PCR }\end{array}\end{array}$

Matched small

Keck 2018

bowel tissue,

RNA sequencing, Whole transcriptome

primary SI-NETs, microarrays, qPCR

liver metastases

Simbolo $2018 \quad 52 \quad$ SI-NETs

High-coverage target sequencing, qPCR, $\mathrm{FISH}$, expression analysis of SRC gene, immunohistochemistry

89

SI-NETs (small intestine,

Yao 2019 jejunum, duodenum, cecum, CUP)

Analysis included CUP and cecum NETs

${ }^{*}$ Chr, chromosome; CIMP, CpG island methylator phenotype; CUP, cancer of unknown primary; DASL, CDNA-mediated Annealing, Selection, extension and Ligation; GI-NET, gastrointestinal NET; $L O H$, loss of heterozygosity; NGS, next-generation sequencing; OR, odds ratio; PFS, progression-free survival; $S N V$, somatic single variants; WT, wildtype; MPTR: mean metastatic/primary tumours ratio 
CDKN1B mutations in $10.9 \%$. No clear association was found between $C D K N 1 B$ mutation and protein expression
A trend towards shorter overall survival associated with low expression of CDKN1Bwas observed (multivariate hazards ratio, $2.04 ; 95 \% \mathrm{Cl} 1.06$ 3.93; $P=0.03)$. CDKN1Bmutation was not associated with survival.

Chr $18 \mathrm{LOH}$ in 65\%. Only DCC (deleted in colorectal cancer) revealed loss of/greatly reduced expression in $29 \%$. No additional genetic or epigenetic alterations were present on Chr18

Serial differential expression was validated in $7 / 10$ genes, with several interacting members of the $A K T, M Y C$, or MAPK3 pathways. Liver metastases had underexpression of PMP22 $(P<0.001)$ High expression of SERPINA10 (primary $\mathrm{p}<0.001$, liver metastases $<0.001$ ) and SYT13(primary $P<0.001$, liver metastases $<0.001$ ) was characteristic of primary SINETs and liver metastases

Mutations in CDKN1B (9.6\%), APC and CDKN2C(each $7.7 \%$ ), BRAF, KRAS, PIK3CA and TP53 (each 3.8\%). Frequent allelic loss of 4 genes located on Chr 18 (BCL2, CDH19, DCC and SMAD4) in $44.2 \%$ and losses on chromosomes $11(38 \%)$ and $16(15 \%)$. Gains on chromosomes $4(31 \%), 5(27 \%), 14(36 \%)$, and 20 $(20 \%)$

BCOR (5.6\%) and CDKN1B (4.5\%) most frequently mutated genes. $\mathrm{LOH}$ was present in approximately $50 \%$ and copy number gains of Chr 4,5,14 and 20 in $>25 \%$. Five distinct genomic clusters were identified (cluster 1: LOH of Chr 18, cluster 2: gain Chr 5 and 7, cluster 3: gain Chr 4,5,14 and 20, cluster 4: Chr 4,5,7,14 and 20, cluster 5: copy number gains across most chromosomes)
Loss of CABLES did not correlate with survival
SRC gene copy number gains were associated with a poorer prognosis $(P=0.0047)$
Lower generalised chromosomal instability in SI-NETs $(n=55)$ was associated with longer survival compared to high CIN ( $n=38)$ with a PFS of 18.6 vs 9.2 months (HR $0.41 ; 95 \% \mathrm{Cl} 0.24$ $0.73 ; P=0.0021)$ 
metastases than in primary tumours..$^{33,43,44}$ In addition, increased hypermethylation of the CTNNB1 promoter was observed in liver metastasis compared to the corresponding primary tumours. ${ }^{44}$ Promotor gene methylation was also found in a study by Bollard et al. $(n=38)$ in $96 \%$ of ileal NETs and their metastases. The expression of the axon guidance molecule SEMA3F (semaphorin 3F) was lost due to hypermethylation. ${ }^{45}$ SEMA3F expression is a negative regulator of MAPK and mTOR signalling pathways.

The first genome-wide DNA methylation analysis of SI-NETs performed by Verdugo et al. in 10 SI-NETs and ten matched mesenteric lymph node metastasis observed a high level of methylation in another gene set located at chromosome $18 q 21$-qter. ${ }^{46}$ In these patients, high methylation index correlated with more malignant behaviour.

Karpathakis et al. found hypermethylation of the promoter region of the gastric inhibitory polypeptide receptor (GIPR; inhibits gastric secretion and gastrin release and stimulates insulin release) gene body in $74 \%$ of primary SI-NETs. Of note, in this study DNA methylation in SI-NETs was compared to the methylation status of normal intestinal mucosa, which normally expresses GIPR, whereas the methylation status of enterochromaffin cells in the small intestine is unknown. Progressive hypermethylation of this gene was seen in liver metastases compared to primary tumours. ${ }^{29,31}$

MicroRNAs in primary tumours vs. metastases

Two miRNA profiling studies ( $n=8, n=24$, respectively) comparing primary SI-NETs to its respective metastases found multiple miRNAs to be deregulated during tumour progression. ${ }^{40,47}$ A downregulation of miRNA-133a and upregulation of miR-183 was consistently found in metastases vs primaries. A study by Miller et al. $(n=28)$ confirmed downregulation of miRNA133a and found differential expression of several other miRNAs in SI-NETs and their metastases. ${ }^{48}$

\section{Discussion}

SI-NETs are rare tumours with a relatively indolent course. Unfortunately, treatment options are limited with minimal survival benefit. Therapies targeting somatostatin receptors, expressed by the majority of SI-NETs, are only able to stagnate disease 
progression temporarily. In an attempt to identify prognostic factors and new effective targets for precision medicine, the genomic landscape of SI-NETs has been under increasing investigation in recent years. $\mathrm{LOH}$ at chromosome 18 remains the most frequent genomic aberration (44-100\%) found in SI-NETs. ${ }^{11-22}$

The tumour suppressor gene, CDKN1B, is mutated in approximately $8 \%$ patients with a SI-NET. ${ }^{11,15,26-29}$ Interestingly, CDKN1B is regulated by menin, the protein that is defect in the majority of patients with the inheritable MEN 1 syndrome (75-80\%). Moreover, in MEN1 patients without mutations in the gene encoding menin (20$25 \%)$, CDKN1B was shown to be inactivated in some individuals (3.6\%). ${ }^{49}$ Thus, several SI-NETs and MEN1-associated endocrine tumours may share a common oncogenic pathway. Genetic alterations in the P13K/AKT/mTOR were found in primary SI-NETs and liver metastasis, providing a rationale for the use of mTOR inhibitors. ${ }^{15,23,31,45}$ However, a correlation between efficacy and mTOR mutational status prior to commencement of therapy with mTOR inhibitors has not yet been established. Daskalakis et al. $(n=27)$ recently tested the ex vivo activity of several targeted kinase inhibitors and found great variability in ex vivo sensitivity for most drugs, emphasising the need for predictive biomarkers which could support clinical decision making. ${ }^{50}$

Furthermore, mutations in APC, CDKN2C (both 7.7\%) and BRAF, KRAS, PIK3CA and TP53 (each 3.8\%) were recently identified in SI-NETs. ${ }^{15}$

An association of (epi)genetic aberrations with prognosis was found in 16 of the 35 original studies reviewed. Karpathakis et al. $(n=97)$ and Yao et al. $(n=89)$ identified molecular subtypes of SI-NETs with significant difference in PFS. ${ }^{11,31}$ However, validation of these subgroups in an independent and larger cohort is required before translation into clinical practice is possible. A gain of chromosome 14 and 20pterp11.21 was associated with shorter survival in two studies $(\mathrm{P}<0.001, \mathrm{P}<0.013$ respectively). ${ }^{13,14}$ SRC copy number gains were associated with poorer prognosis $(P=0.047)(11)$. Epigenetic alterations such as specific promotor methylation and global methylation and their effect on prognosis are yet to be determined. ${ }^{33,41,43-46}$

At present, predictive or prognostic biomarkers, which can be adopted into clinical practice, have not yet been established. Inactivated tumour suppressor genes, which are found in SI-NETs, are generally unsuitable as targets since restoring the function of tumour suppressor genes is difficult to accomplish. Mutations in oncogenes, which should be easier to target, have only recently been described in small numbers in SI- 
NETs and thus far no clinical studies have been undertaken to target these mutations in SI-NETs. Of note, Alvarez et al. identified the HDAC class inhibitor Etinostat as potent inhibitor of master regulatory activity for $42 \%$ of metastatic gastroenteropancreatic NETs, leading to the initiation of a clinical trial (NTC03211988). ${ }^{9}$

The low mutational burden found in SI-NETS may render these tumours less eligible for immunotherapy using immune checkpoint inhibitors because tumour mutational burden is an important determinant of clinical benefit to immune checkpoint blockade in most tumours. Additionally, the recently characterised tumour microenvironment in NETs, for example low expression for PD1 and PDL1 in SINETs, combined with a modest T-cell infiltrate, further tempers expectations regarding a response to the currently used PD1 and PDL-1 inhibitors, although this remains to be investigated. ${ }^{51}$

More promising targets in SI-NETs may constitute the DNA methylation machinery. In comparison with genetic mutations, epigenetic alterations are significantly more common in SI-NETs. Specific genes such as RASSF1A, SEMA3F and CTNNB1 are hypermethylated in SI-NETs silencing their transcription. ${ }^{3,43-46}$ RASSF1A hypermethylation is also observed in pancreatic NETs, lung NETs and thymic NETs, whilst it is not found in appendiceal NETs. ${ }^{52}$ During the last decades, an increasing number of drugs targeting DNA methylation and histone methylation have been developed and successfully tested pre-clinically which are currently in evaluation in phase I-III clinical trials. ${ }^{53}$ Additionally, the more specific upregulation of miRNAs in SI-NETS as described above may provide actionable targets since multiple strategies for miRNA-based therapies are under investigation. ${ }^{54}$

In this era of accumulating studies regarding the molecular background of SI-NETs, we felt there was an unmet need to provide the clinician with an overview of (epi)genetic alterations and explain their relevance in terms of prognosis and possible novel therapeutic options. Despite our efforts to perform an extensive and broad search, studies may have been missed due to its non-systemic character. Limitations of studies used in this review include relatively small and heterogeneous cohorts, different genomic analysis techniques and paucity of relation of (epi)genetic aberrations to clinical outcomes. 
The rarity of SI-NETs has hampered conducting sizeable clinical trials involving large-scale integrated genomic analysis. In the coming years, hopefully international collaborations will enable larger studies to be performed which correlate (epi)genetic alterations to clinical outcomes and aim to identify targetable (epi)genetic alterations. Larger studies combined with evolving molecular technologies might lead to a more effective treatment strategy in which patients with specific molecular tumour profiles will be selected for targeted pharmaceutical interventions.

\section{Conclusion}

SI-NETs have a low mutational burden, whereas epigenetic alterations are more prevalent. Mutations as described in pancreatic NETs are generally not observed in SI-NETs. Several studies identified (epi)genetic subtypes and molecular profiles of SINETs with significant difference in progression-free survival (PFS) and overall survival (OS). More research should be conducted to identify prognostic and predictive biomarkers that can be adopted in clinical decision-making. 


\section{References}

1. Yao JC, Hassan M, Phan A, et al. One hundred years after 'carcinoid': epidemiology and prognostic factors for neuroendocrine tumors in 35,825 cases in the United States. Journal of Clinical Oncology 2008;26:3063-3072.

2. Hallet J, Law $\mathrm{CH}$, Cukier M, et al. Exploring the rising incidence of neuroendocrine tumors: a population-based analysis of epidemiology, metastatic presentation, and outcomes. Cancer 2015;121:589-597.

3. Garcia-Carbonera R, Jilmenez-Fonseca P, Teulé A, et al. SEOM clinical guidelines for the diagnosis and treatment of gastroenteropancreatic neuroendocrine neoplasms (GEP-NENs) 2014. Clinical and Translational Oncology 2014;16:1025-1034.

4. Hassan SA, Banchs J, Iliescu C, et al. Carcinoid heart disease. Heart 2017;103:14881495.

5. Dasari A, Shen C, Halperin D, et al. Trends in the incidence, prevalence, and survival outcomes in patients with neuroendocrine tumors in the United States. JAMA Oncology 2017;3:1335-1342.

6. Pan SY, Morrisson H. Epidemiology of cancer of the small intestine. World Journal of Gastrointestinal Oncology 2011;3:33-42.

7. Zatelli MC, Fanciulli G, Malandrino P, et al. Predictive factors of response to mTOR inhibitors in neuroendocrine tumours. Endocrine-Related Cancer 2016;23:R173R183.

8. Singh S, Carnaghi C, Buzzoni R, et al. Everolimus in neuroendocrine tumors of the gastrointestinal tract and unknown primary. Neuroendocrinology 2018;106:211-220.

9. Alvarez MJ, Subramaniam PS, Tang LH, et al. A precision oncology approach to the pharmacological targeting of mechanistic dependencies in neuroendocrine tumors. Nature Genetics 2018;50:979-989.

10. Scarpa A, Chang DK, Nones K, et al. Whole-genome landscape of pancreatic neuroendocrine tumours. Nature 2017;543:65-71.

11. Yao JC, Garg A, Chen D, et al. Genomic profiling of NETs: a comprehensive analysis of the RADIANT trials. Endocrine-Related Cancer 2019;26:391-403.

12. Kulke MH, Freed E, Chiang DY, et al. High-resolution analysis of genetic alterations in small bowel carcinoid tumor reveals areas of recurrent amplification and loss. Genes, Chromosomes and Cancer 2008;47:591-603.

13. Andersson E, Swärd C, Stenman G, et al. Highresolution genomic profiling reveals gain of chromosome 14 as a predictor of poor outcome in ileal carcinoids. EndocrineRelated Cancer 2009;16:953-966. 
14. Hashemi J, Fotouhi O, Sulaiman L, et al. Copy number alterations in small intestinal neuroendocrine tumors determined by array comparative genomic hybridization. BMC Cancer 2013;13:505.

15. Simbolo M, Vincentini C, Maffacini A, et al. Mutational and copy number asset of primary sporadic neuroendocrine tumors of the small intestine. Virchows Archiv 2018;473:709-717.

16. Löllgen RM, Hessman O, Szabo E, et al. Chromosome 18 deletions are common events in classical midgut carcinoid tumors. International Journal of Cancer 2001;92:812-815.

17. Wang GG, Yao JC, Worah S, et al. Comparison of genetic alterations in neuroendocrine tumors: frequent loss of chromosome 18 in ileal carcinoid tumors. Modern Pathology 2005;18:1079-1087.

18. Kim DH, Nagano Y, Choi IS, et al. Allelic alterations in well-differentiated neuroendocrine tumors (carcinoid tumors) identified by genome-wide single nucleotide polymorphism analysis and comparison with pancreatic endocrine tumors. Genes, Chromosomes and Cancer 2008;47:84-92.

19. Cunningham JL, Díaz de Ståhl T, Sjöblom T, et al. Common pathogenetic mechanism involving human chromosome 18 in familial and sporadic ileal carcinoid tumors. Genes, Chromosomes and Cancer 2011;50:82-94.

20. Delgado Verdugo A, Crona J, Maharjan R, et al. Exome sequencing and CNV analysis on chromosome 18 in small intestinal neuroendocrine tumors: ruling out a suspect? Hormone and Metabolic Research 2014;47:452-455.

21. Andersson E, Arvidsson Y, Swärd C, et al. Expression profiling of small intestinal neuroendocrine tumors identifies subgroups with clinical relevance, prognostic markers and therapeutic targets. Modern Pathology 2016;29:616-629.

22. Nieser M, Henopp T, Brix J, et al. Loss of chromosome 18 in neuroendocrine tumors of the small intestine: the enigma remains. Neuroendocrinology 2017;104:302-312.

23. Banck MS, Kanwar R, Kulkarni AA, et al. The genomic landscape of small intestine neuroendocrine tumors. Journal of Clinical Investigation 2013;123:2502-2508.

24. Francis JM, Kiezun A, Ramos AH, et al. Somatic mutation of CDKN1B in small intestine neuroendocrine tumors. Nature Genetics 2013;45:1483-1486.

25. Edfeldt K, Ahmad T, Akerström G, et al. TBEB3C a putative tumor suppressor gene of small intestinal neuroendocrine tumors. Endocrine-Related Cancer 2014;21:275-284.

26. Crona J, Gustavsson T, Norlén O, et al. Somatic mutations and genetic heterogeneity at the CDKN1B locus in small intestinal neuroendocrine tumors. Annals of Surgical Oncology 2015;22:S1428-S1435. 
27. Shi Y, Qian ZR, Zhang S, et al. Cell cycle protein expression in neuroendocrine tumors. Pancreas 2017;46:1347-1353.

28. Maxwell JE, Sherman SK, Li G, et al. Somatic alterations of CDKN1B are associated with small bowel neuroendocrine tumors. Cancer Genetics 2015:208:564-570.

29. Karpathakis A, Dibra H, Pipinikas C, et al. Prognostic impact of novel molecular subtypes of small intestinal neuroendocrine tumor. Clinical Cancer Research 2016;22:250-258.

30. Bottarelli L, Azzoni C, Pizzi S, et al. Adenomatous polyposis coli gene involvement in ileal enterochromaffin cell neuroendocrine neoplasms. Human Pathology 2013;44:2736-2742.

31. Karpathakis A, Dibra H, Pinnikas C, et al. Progressive epigenetic dysregulation in neuroendocrine tumour liver metastases. Endocrine-Related Cancer 2017;24:L21L25.

32. Keck KJ, Breheny P, Braun TA, et al. Changes in gene expression in small bowel neuroendocrine tumors associated with progression to metastases. Surgery 2018;163:232-239.

33. Fotouhi O, Abdel Fahmideh M, Kjellman M, et al. Global hypomethylation and promoter methylation in small intestinal neuroendocrine tumors. Epigenetics 2014;9:987-997.

34. Edfeldt K, Björklund P, Akerstörm G, et al. Different gene expression profiles in metastasizing midgut carcinoid tumours. Endocrine-Related Cancer 2011;18:479489.

35. Di Domenico A, Wiedmer T, Marinoni I et al. Genetic and epigenetic drivers of neuroendocrine tumors (NET). Endocrine-Related Cancer 2017;24:R315-R334.

36. Dumanski JP, Rasi C, Björklund P, et al. A MUTYH germline mutation is associated with small intestinal neuroendocrine tumours. Endocrine-Related Cancer 2017;24:427-443.

37. Raj N, Shah R, Stadler Z, et al. Real-time genomic characterization of metastatic pancreatic neuroendocrine tumors has prognostic implications and identifies potential germline actionability. JCO Precision Oncology 2018;2018:1-18.

38. Holliday R. The inheritance of epigenetic defects. Science 1987;238:163-170.

39. Stålberg P, Westin G, Thirlwell C. Genetics and epigenetics in small intestinal neuroendocrine tumours. Journal of Internal Medicine 2016;280:584-594.

40. Ruebel K, Leontovich A, Stilling G, et al. MicroRNA expression in ileal carcinoid tumours: downregulation of microRNA-133a with tumor progression. Modern Pathology 2009;23:367-375. 
41. Stricker I, Tzivras D, Nambiar S, et al. Site- and grade-specific diversity of LINE1 methylation pattern in gastroenteropancreatic neuroendocrine tumours. Anticancer Research 2012;32:3699-3706.

42. Finnerty BM, Gray KD, Moore MD, et al. Epigenetics of gastroenteropancreatic neuroendocrine tumors: a clinicopathologic perspective. World Journal of Gastrointestinal Oncology 2017;9:341.

43. Choi IS, Estecio MR, Nagano Y, et al. Hypomethylation of LINE-1 and Alu in welldifferentiated neuroendocrine tumors (pancreatic endocrine tumors and carcinoid tumors). Modern Pathology 2007;20:802-810.

44. Zhang $\mathrm{H}$, Rumilla KM, Jin $\mathrm{L}$, et al. Association of DNA methylation and epigenetic inactivation of RASSF1A and beta-catenin with metastases in small bowel carcinoid tumors. Endocrine 2006;30:299-306.

45. Bollard J, Massoma P, Vercherat C, et al. The axon guidance molecule semaphorin 3F is a negative regulator of tumor progression and proliferation in ileal neuroendocrine tumors. Oncotarget 2015;6:36731-36745.

46. Verdugo AD, Crona J, Starker L, et al. Global DNA methylation patterns through an array-based approach in small intestinal neuroendocrine tumors. Endocrine-Related Cancer 2014;21:L5-L7.

47. Li S, Essaghir A, Martijn C, et al. Global microRNA profiling of well-differentiated small intestinal neuroendocrine tumours. Modern Pathology 2013;26:685-696.

48. Miller HC, Frampton AE, Malczewska A, et al. MicroRNAs associated with small bowel neuroendocrine tumours and their metastases. Endocrine-Related Cancer 2016;23:711-726.

49. Georgitsi M, Raitila A, Karhu A, et al. Germline CDKN1B/p27Kip1 mutation in multiple endocrine neoplasia. Journal of Clinical Endocrinology and Metabolism 2007;92:3321-3325.

50. Daskalakis K, Norlén O, Karakatsanis A, et al. Ex vivo activity of cytotoxic drugs and targeted agents in small intestinal neuroendocrine tumors. Endocrine-Related Cancer 2018;25:471-480.

51. Da Silva A, Bowden M, Zhang S, et al. Characterization of the neuroendocrine tumor immune microenvironment. Pancreas 2018;47:1123-1129

52. Karpathakis A, Dibra H, Thirwell C. Neuroendocrine tumours: cracking the epigenetic code. Endocrine-Related Cancer 2013;20:R65-R82.

53. Ahuja N, Sharma AR, Baylin SB. Epigenetic therapeutics: a new weapon in the war against cancer. Annual Review of Medicine 2016;67:73-89. 
Part I | Chapter 3

54. Chakraborty C, Sharma AR, Sharma G, et al. Therapeutic miRNA and siRNA: moving from bench to clinic as next generation medicine. Molecular Therapy: Nucleic Acids 2017;8:132-143.

55. Hanahan D, Weinberg R, Hallmarks of cancer: the next generation. Cell 2011;144:646-674.

56. Walsh K, Choi M, Oberg K, et al. A pilot genome-wide association study shows genomic variants enriched in the nontumor cells of patients with well differentiated neuroendocrine tumors of the ileum. Endocrine-Related Cancer 2010;18:171-180.

57. Norlén O, Edfeldt K, Akerstörm G, et al. Peritoneal carcinomatosis from small intestinal neuroendocrine tumors: clinical course and genetic profiling. Surgery 2014;156:1512-152. 



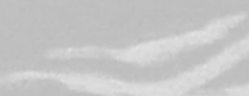

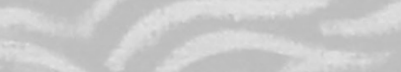

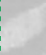

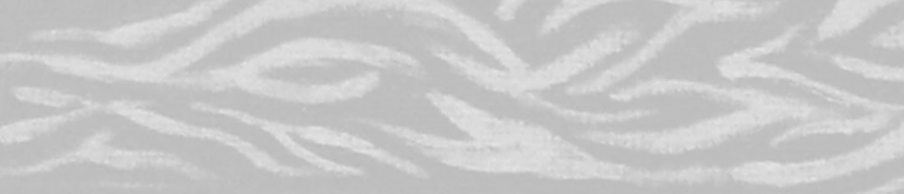




\section{Chapter 4}

\section{Merkel cell carcinoma: clinical outcome and prognostic factors in 351 patients}

L.M. van Veenendaal, A.C.J. van Akkooi, C. Verhoef, D.J. Grünhagen, W.M.C. Klop, G.D. Valk, M.E.T. Tesselaar

Journal of Surgical Oncology, 2018 


\section{Abstract}

\section{Background}

Merkel cell carcinoma (MCC) is a rare and aggressive neuroendocrine carcinoma of the skin.

\section{Aim}

To describe clinical outcome and prognostic factors of MCC patients in two expertcenters.

\section{Patients and Methods}

Patients with histologically confirmed MCC in 1990-2014 were included. Data on patient, tumour characteristics and treatment were retrospectively collected.

\section{Results}

A total of 351 Patients were evaluated, 153 (44\%) males, median age 74 years (range 28-94). Median follow-up time was 28 months (IQR 13-58). Median primary tumour size was $17 \mathrm{~mm}$ (range 2-135). At time of diagnosis 112 (32\%) patients had lymph node metastases. The cohorts' 5 -year overall survival (OS) was 58\%. Using a competing risk analysis the 5 -year relapse and MCC related death was $42 \%$ and $22 \%$. Post-operative radiation therapy (RT) was associated with reduced recurrence (SDH 0.54; $\mathrm{Cl} 0.3-0.9)$. Nodal involvement (SDH 2.7; $\mathrm{Cl} 1.1-6.6)$ and the male gender were associated with higher $\mathrm{MCC}$ related death $(\mathrm{SDH} 3.1 ; \mathrm{Cl} 1.2-7.9)$

\section{Conclusion}

In a large cohort a low MCC related death, in the presence of a low OS was seen. This indicates that a significant number of MCC patients die due to other causes than MCC. Post-operative RT was associated with relapse. Male gender and nodal metastasis were associated with MCC related death. 


\section{Introduction}

Merkel cell carcinoma (MCC) is a rare and aggressive neuroendocrine carcinoma of the skin. It affects mostly elderly Caucasian patients, and males slightly more frequent than females. The predominant locations are the head and neck region, followed by the extremities. ${ }^{1}$ These sun - exposed locations support the assumption that ultraviolet exposure increases the risk of $\mathrm{MCC}^{2}$ Other factors associated with an increased risk are immunosuppression and Merkel cell Polyomavirus (MCPyV). ${ }^{3}$ In rare cases no primary tumour can be found, similarly to melanoma which it is often compared to. Possibly, the immune system is responsible in initiating regression of the primary tumour. ${ }^{4}$

MCC is characterised by an aggressive metastatic spread. At diagnosis, approximately $26 \%$ of the patients have nodal involvement. Loco-regional recurrence is seen in 26 $60 \%$, often within 2 years after diagnosis. ${ }^{5,6}$ Overall survival ranges from 30 to $60 \% .^{7,8}$ The rapid spread and poor survival of MCC justifies an aggressive approach in its treatment, which consists mainly of surgical treatment followed by postoperative radiotherapy (RT) for early stage MCC. ${ }^{9,10}$ Chemotherapy is indicated only for patients with distant metastases, with a quick but often short response. ${ }^{11,12}$ Adjuvant chemotherapy is not of benefit. ${ }^{13}$ Immunotherapy has promising results but has not established a definitive role in the treatment of MCC yet. ${ }^{14}$ In the last decade, the reported incidence has increased rapidly. ${ }^{15-19}$ This may be partly explained by better recognition and uniform staining but also increased registration as an unique entity.

Literature on MCC is quite limited. Most studies are retrospective and single center. ${ }^{13,20,21}$ Several large database series using the Surveillance, Epidemiology, and End Results program (SEER) and the National Cancer Data Base (NCDB) investigated MCC. Unfortunately, these series often lacked data on treatment, recurrence, and cause of death. 5,7,9,22,23 The aims of the current study are to describe treatment, relapse, overall survival (OS), and more specifically, MCC related death of patients with MCC in two large melanoma and neuroendocrine tumour expert-centers in the Netherlands. 


\section{Patients and methods}

This study was performed in accordance with institutional ethical guidelines in both clinics. All patients with histologically confirmed MCC between January 1990 and December 2014 were included. Patients were identified using the clinics' tumour registry administration. A retrospective review of each patient's medical record was completed. Patients were excluded from analysis when MCC was not histologically confirmed and information on primary treatment was missing. Patients' and disease characteristics were collected. Patient's work-up consisted of imaging modalities according to physicians' choice; mostly CT or PET/CT. Pathological reports were used to collect tumour characteristics. If primary tumour size (PTS) could not be collected from the reports the PTS, clinically determined by a dermatologist, was used. A resection margin was considered negative irrespective of the width (close, $<1 \mathrm{~cm}$ or $2 \mathrm{~cm}$ ) and positive in case of microscopically detectable MCC. Treatment characteristics and recurrence were recorded. Surgery included (wide) local excision and Moh's surgery Lymph node dissection (LND) included dissection of the parotid gland, selective or modified radical lymph node dissection of neck, superficial or deep lymph node dissection of the groin and lymph node dissections of the axilla. Local recurrence of the primary tumour was defined as recurrence within the scar or described by the clinician as such. In-transit (IT) recurrence was defined as a cutaneous or subcutaneous metastasis between the primary location and tumour draining nodal basin. Patients without documented nodal staging at presentation and nodal recurrence within 3 months were considered to have nodal disease at time of diagnosis. Nodal recurrence was defined as recurrence of nodal disease irrespective the interval when nodal staging at diagnosis was performed, and $>3$ months without nodal staging at diagnosis. For analysis, recurrence of primary tumour, IT and regional lymph nodes were combined into loco - regional recurrence. Distant recurrence consisted of recurrence of MCC in distant lymph node or organs. Followup, vital status and cause of death were recorded.

- Statistical analysis

Data were summarized using standard descriptive statistics: frequency and percentage for categorical variables, mean with standard deviation (SD) or median with interquartile range (IQR) and range for continuous variables. Endpoints were relapse; OS and MCC related death, which were calculated from date of diagnosis. Patients alive before reaching one of the endpoints were censored at their last time of 
follow-up. OS was analysed with a Kaplan Meier and log-rank test for univariable analysis and a Cox proportional hazards for multivariable analysis. For relapse and MCC related death, competing risk regression models were used assuming proportional hazards. Cumulative incidence curves were univariable compared using Gray's test and the Fine-Gray sub distributional hazard model was used to perform a multivariable analysis. Patients who died of other causes (DOC) or unknown causes (DU) when estimating MCC related death or relapse were treated as competing risks. Patients who died of MCC (DOD) were considered an event when relapse was analysed. Age was analysed as a continuous variable in multivariable analyses. Associations were summarized by calculating hazard ratios (HR), sub distribution $H R$ $(\mathrm{SDH})$, and corresponding 95\% confidence intervals $(\mathrm{Cl})$. Calculated $\mathrm{P}$ values were two - sided and <.05 were considered statistically significant. Analyses were performed using SPSS software version 24 (SPSS Inc., Chicago, IL) and R version 3.4.1 an open source statistical software (www.r-project.org).

\section{Results}

We identified 360 patients of which nine patients were excluded from analysis according exclusion criteria. The cohort consisted of 153 male (44\%) and 198 female (56\%) patients with a median age of 74 years (IQR 28-94). Baseline characteristics are summarized in Table 1. A total of 52 patients (15\%) were immune compromised of which $7 \%$ due to autoimmune disorders, $6 \%$ due to hemato-oncological malignancies, $2 \%$ due to previous organ transplantation and one patient because of an HIV infection. A total of 104 patients (30\%) had previous malignancies. Medical history and comorbidities are more specifically outlined in Table 1.

MCC was primarily located in the head and neck $(\mathrm{H} / \mathrm{N})$ in 172 patients $(49 \%)$. PTS was documented in 210 patients (60\%) (median $17 \mathrm{~mm}$, IQR 2-135). Taking patients with an unknown primary into account, $31 \%$ had no registration of PTS. At the time of diagnosis loco-regional metastases were present in 112 patients (32\%) and distant metastases in ten patients $(3 \%)$. Metastases were confirmed by pathological examination in $95 \%$. Imaging confirmed the presence of metastases in three patients $(2,5 \%)$. Three patients $(2,5 \%)$ with in-transit disease metastases were diagnosed clinically. A total of four patients without documented nodal staging at diagnosis and recurrence within 3 months were classified as having nodal disease at diagnosis. 
Part I | Chapter 4

Table 1: Baseline characteristics

\begin{tabular}{|cr} 
Characteristic & $\begin{array}{r}\text { n (\%) or median } \\
\text { (IQR, range) }\end{array}$ \\
\hline \hline Gender: Male & $153(44 \%)$ \\
Female & $198(56 \%)$ \\
\hline Age (years) & $74(16,28-94)$ \\
$\mathbf{6 0}$ years & $50(14 \%)$ \\
$\mathbf{6 0 - 6 9}$ years & $86(25 \%)$ \\
$\mathbf{7 0 - 7 9}$ years & $105(30 \%)$ \\
$\mathbf{8 0}$ years & $110(31 \%)$
\end{tabular}

Medical history and comorbidities:

Melanoma

$13(4 \%)$

Other malignancies:

$104(30 \%)$

Non-melanoma skin cancer

$43(12 \%)$

Hemato-oncological neoplasias

$21(6 \%)$

Cardiovascular disorders

$135(39 \%)$

Pulmonary disorders

$16(5 \%)$

Neurological disorders

$28(8 \%)$

Auto-immune disorders:

$24(7 \%)$

Organ transplantation

$6(2 \%)$

HIV

$1(0.3 \%)$

Data on primary tumour size

$210(60 \%)$

Primary tumour size ( $\mathrm{mm}$ )

$17(15,2-135)$

Location: Head and neck

$172(49 \%)$

Extremities

$107(31 \%)$

Torso

$40(11 \%)$

Unkown primary

$32(9 \%)$

Metastases at diagnosis:

Locoregional metastases

$112(32 \%)$

Distant metastases

$10(3 \%)$

Stage I

$110(31 \%)$

Stage II

$40(11 \%)$

Stage III

$112(32 \%)$

Stage IV

$10(3 \%)$

Staging unavailable

$79(23 \%)$

* according AJCC Staging Manual 8th edition 
Patients with MCC underwent various treatment regimens (Figure 1). In general, in case of resectable loco-regional disease surgery was performed with or without postoperative RT. The use of RT was indicated in case of a positive or close margin and positive nodes. A total of 325 patients underwent surgery. Of these patients $24(7 \%)$ underwent a LND and 301 patients (93\%) excision of the primary tumour. A resection margin was reported in 274 patients (78\%). A clear margin was obtained in 225 patients (64\%), and a positive margin was seen in 49 patients (14\%). A reexcision was performed in 148 patients (46\%) due to a positive or a close (but clear) margin. In 86 patients (29\%) an excision was combined with a sentinel lymph node biopsy (SLNB). The SLNB proved to be positive in 28 patients (33\%) after which a LND was performed in 21 patients $(75 \%)$ combined with RT in six patients (21\%). A total of 120 patients (34\%) received RT, either post-operative RT in 111 patients $(93 \%)$ or as definitive treatment in nine patients $(7 \%)$.

A total of 42 Patients (35\%) received RT due to nodal disease, and 68 patients (57\%) due to a close or positive margin. Another ten patients (8\%) received post-operative RT without nodal disease but their margin status was unknown. Fifty-four patients (45\%) received RT at the primary tumour and in 20 patients $(17 \%)$ on regional lymph nodes. In 36 patients (30\%) both regions were radiated. One patient received RT on distant metastases. In eight patients (7\%) no description of the radiation field was given. The median administered dose was 60 Gy (IQR 50-60) in 25 fractions (IQR 20-30). Chemotherapy was given in 19 patients (5\%). Two patients received chemotherapy prior to surgery, six patients adjuvant chemotherapy, and eight patients as definitive therapy. Patients who received chemotherapy, either primary or (neo) adjuvant, were all given platinum based regimens. Another 13 patients (4\%) received chemo-radiation (CXRT) as neo-adjuvant treatment in five patients and definitive treatment in eight patients, respectively. One patient $(0.3 \%)$ underwent Isolated Limb Perfusion (ILP). 


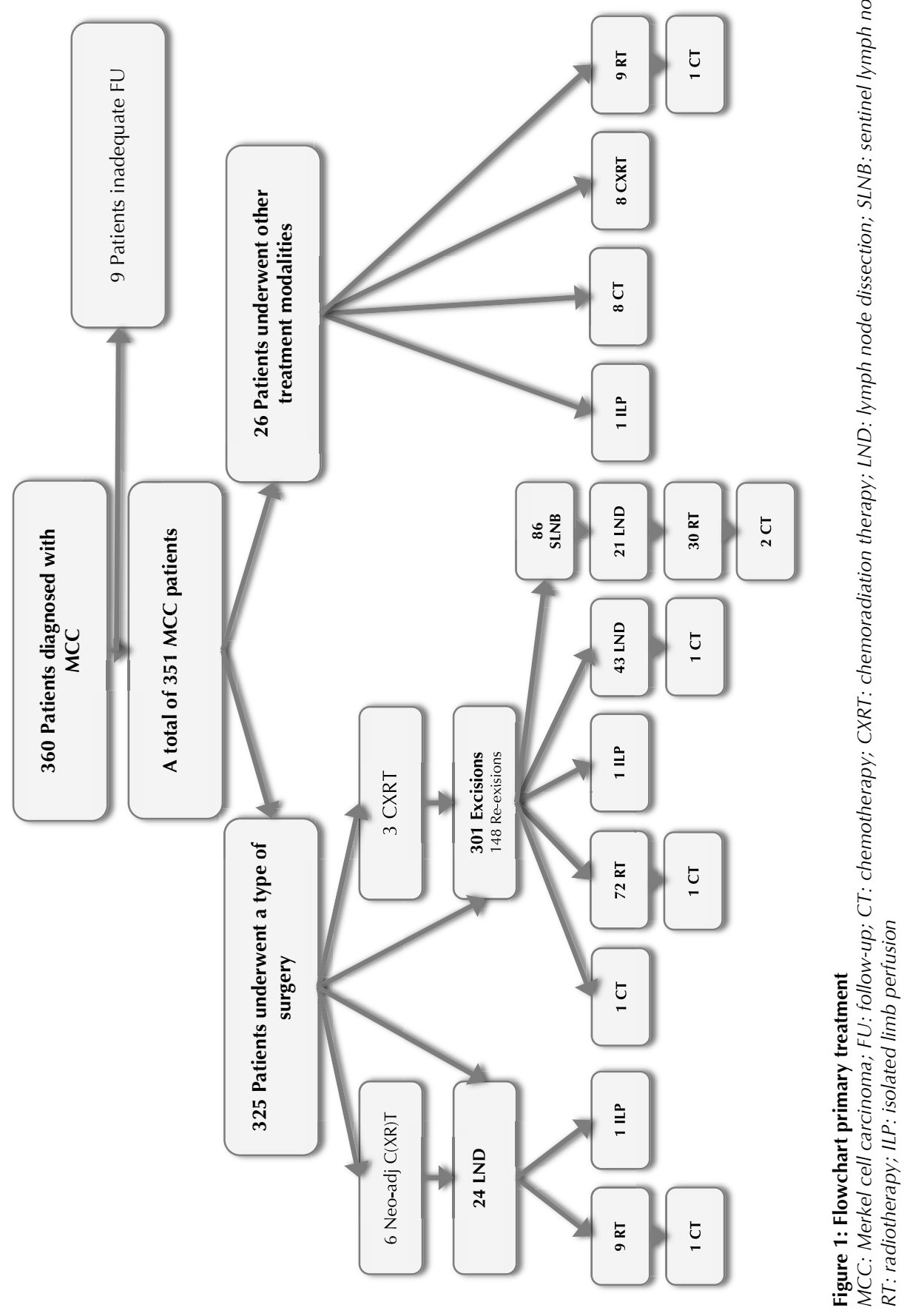


Median follow-up (FU) for the whole cohort was 28 months (IQR 13-58). Deceased patients at the end of follow-up had a median FU of 19 months (IQR 10-45) and surviving patients had a median FU of 35 months (IQR 19-64). At last FU 58 patients (17\%) had died of MCC. In 64 patients (18\%) death occurred due to other causes. In seven patients ( $2 \%$ ) no cause of death was registered. Of these patients four patients had nodal involvement and two patients had distant metastases. The remaining patient had localized disease only. Two patients wished to be withdrawn from FU and one patient transferred to another clinic due to urgent cardiac co-morbidity. Of the patients still alive at last FU, 168 patients (48\%) had no evidence of disease and 54 patients $(15 \%)$ were alive with disease (Table 2$)$.

Table 2: Vital status at end of follow-up

\begin{tabular}{lr}
\hline Vital status & $\mathbf{n ~ ( \% )}$ \\
\hline \hline Died of MCC & $58(17 \%)$ \\
\hline Died of other causes & $64(18 \%)$ \\
\hline Alive with MCC & $54(15 \%)$ \\
\hline No evidence of MCC & $168(48 \%)$ \\
\hline Cause of death unknown & $7(2 \%)$ \\
\hline MCC: Merkel cell carcinoma & \\
\hline
\end{tabular}

Relapse during FU occurred in 134 patients (38\%) of which 86\% ( $\mathrm{n}=115)$ recurred within 2 years. Relapse occurred most often loco-regional instead of distant metastases, in 93 patients and 41 patients, respectively. The Kaplan-Meier estimated 5 -year OS was 58\%. The 5-year estimated cumulative incidence for relapse and MCC related death was $42 \%$ and $22 \%$, respectively (Graphs 1 ).

All significant univariable analyses on relapse and MCC related death are shown in Graphs 2. (The supplement provides all other univariable analyses) In multivariable analyses post-operative RT was associated with a significant lower relapse (SDH 0.54; Cl 0.3-09). This association was not seen for either OS or MCC related death. Lymph node metastases at diagnoses were associated with relapse $(\mathrm{SDH} 1.9 ; \mathrm{Cl} 1.1$ 3.3) and MCC related death $(\mathrm{SDH} 2.7 ; \mathrm{Cl} 1.1-6.6)$. Gender, age $>74$ years, PTS $>2 \mathrm{~cm}$ and loco - regional metastasis at time of diagnosis were associated with a decreased OS (Table 3). 
Relapse
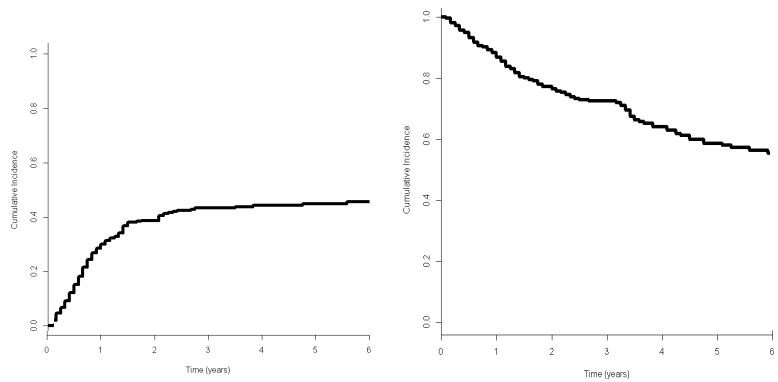

Overall Survival

MCC Related Death

Graphs 1: Relapse and survival curves

RT

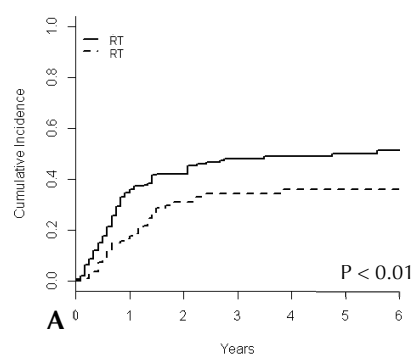

Gender

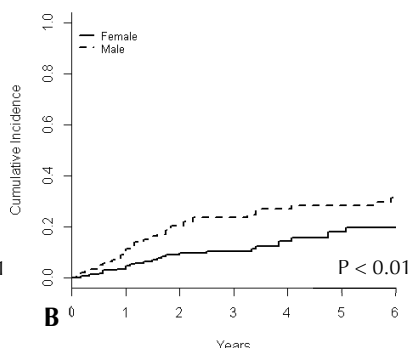

Nodal involvement

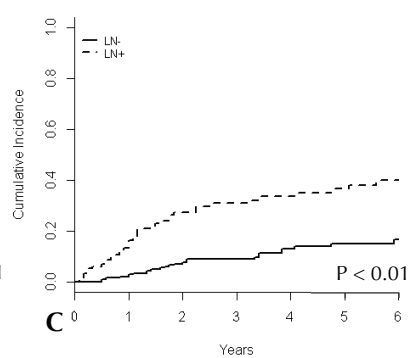

Graphs 2: Univariable significant covariates on relapse (A) and MCC related death (B \& C)

Only patients with a known PTS ( $\mathrm{n}=210,60 \%$ ) could be included in our multivariable analysis. Primary tumour location was not associated with any type of survival. For MCC related death, the male gender (SDH 3.1; $\mathrm{Cl} 1.2-7.9)$ was the strongest independent prognostic predictor (Table 3).

\section{Discussion}

Our study confirms the poor OS and high relapse rate reported in other studies, however we found a low MCC related death rate. This indicates that a significant number of elderly MCC patients die due to other causes than MCC. In the aged MCC population with high comorbidity, the low MCC related death is important to consider when deciding on a therapy strategy. 
Table 3: Overview multivariable analyses

\begin{tabular}{|c|c|c|c|c|c|c|c|c|c|}
\hline \multirow[b]{2}{*}{ Variable } & \multicolumn{3}{|c|}{ Overall Survival* } & \multicolumn{3}{|c|}{ Relapse* } & \multicolumn{3}{|c|}{ MCC Related Death* } \\
\hline & HR & $95 \% \mathrm{Cl}$ & $\boldsymbol{P}$ & SDH & $95 \% \mathrm{Cl}$ & $\boldsymbol{P}$ & SDH & $95 \% \mathrm{CI}$ & $\boldsymbol{P}$ \\
\hline \multicolumn{10}{|l|}{ Gender: } \\
\hline Male & 2.1 & $1.2-3.7$ & 0.01 & 1.1 & $0.6-1.8$ & 0.84 & 3.1 & $1.2-7.9$ & 0.02 \\
\hline Female & ref & & & ref & & & ref & & \\
\hline Age (continous) & 1.1 & $1.0-1.1$ & 0.01 & 1.0 & $0.9-1.0$ & 0.96 & 1.0 & $0.9-1.0$ & 0.59 \\
\hline \multicolumn{10}{|l|}{ PT size: } \\
\hline$<20 \mathrm{~mm}$ & ref & & & ref & & & ref & & \\
\hline$>20 \mathrm{~mm}$ & 1.6 & $0.9-2.7$ & 0.03 & 1.4 & $0.9-2.2$ & 0.15 & 0.6 & $0.4-1.1$ & 0.25 \\
\hline \multicolumn{10}{|l|}{ Localisation: } \\
\hline Head \& Neck & ref & & & ref & & & ref & & \\
\hline Extremities & 0.52 & $0.5-1.6$ & 0.61 & 0.67 & $0.4-1.2$ & 0.17 & 0.55 & $0.23-1.3$ & 0.19 \\
\hline Torso & 0.75 & $0.3-1.9$ & 0.55 & 0.63 & $0.3-1.3$ & 0.23 & 0.63 & $0.19-2.0$ & 0.44 \\
\hline UP & 0.99 & 0.0-inf & 0.99 & 0.44 & $0.1-2.3$ & 0.33 & inf & inf & inf \\
\hline \multicolumn{10}{|l|}{ Metastasis:** } \\
\hline No metastasis & ref & & & ref & & & ref & & \\
\hline Locoregional & 2.1 & $1.1-3.3$ & 0.03 & 1.9 & $1.1-3.3$ & 0.02 & 2.7 & $1.1-6.6$ & 0.03 \\
\hline \multicolumn{10}{|l|}{ Post-operative RT: } \\
\hline None & ref & & & ref & & & ref & & \\
\hline Yes & 1.1 & $0.6-1.8$ & 0.82 & 0.54 & $0.3-0.9$ & 0.02 & 0.9 & $0.4-2.1$ & 0.70 \\
\hline
\end{tabular}

In our cohort post-operative RT is associated with a lower relapse, although without effect on either OS or MCC related death. While local disease control is meaningful for MCC patients, a survival benefit would be desirable. Several factors could have contributed to this result. Firstly, if possible relapsed patients are offered salvage surgery with RT, thus diminishing the effect of primary post-operative RT on survival. Secondly, we have not performed a separate analysis for different MCC stages due to missing PTS. RT has shown a beneficial effect for early stage MCC with and without an effect on survival in other studies as well. ${ }^{10,24,25}$ Harrington et al. showed the (beneficial) effect of post-operative RT irrespective of a close or microscopic positive margin. ${ }^{26}$ Fang et al. showed similar results on relapse rate and survival rate in MCC patients with nodal disease undergoing complete LND with RT and definitive RT. ${ }^{27}$ Although they suggested complete LND could possibly delay relapse. Future research 
could investigate which patients could benefit from a more conservative treatment strategy with the use of RT.

The male gender was our strongest prognostic factor associated with a higher MCC related death rate. Others have seen this relation as well but the exact mechanism remains unknown. ${ }^{9,13,19,28,29}$ Sridharan et al. linked this association to a higher nodal involvement. While Jabbour et al. sought the explanation for the disparity in overall survival in different patient behaviour (e.g. smoking) and delayed presentation, as is often presumed in patients with melanoma. ${ }^{28,31-33}$ However, these explanations seems unlikely since we analysed MCC related death and corrected for size and nodal stage, which would reflect a delayed presentation. Pitale et al. suggested hormone related differences could be accountable for a worse survival. ${ }^{34}$ Though, due to the postmenopausal age of the female MCC population we believe this is not expected. Joosse et al. have shown comparable results in melanoma (considered a similar type of cancer), which remained after correction for all other known prognostic factors. ${ }^{35}$

There is significant evidence that the immune system plays a role in developing MCC and possibly affects survival. MCC is associated with MCPyV and the use of immunosuppressive medication; the immune system is possibly responsible for the spontaneous regression of the primary tumour. ${ }^{3,4}$ Additionally, T-cell density at the periphery of the tumour is associated with an improved OS. ${ }^{36}$ Gender related disparities concerning the immune system are already known, for instance in the prevalence of autoimmune disease. ${ }^{37}$ Hence, we believe the difference in survival between genders could be immune related. More research should aim at explaining the exact mechanism responsible for the worse survival of the male patients.

To our knowledge, our cohort is the second largest institutional study in literature. ${ }^{13}$ 20,28,38-41 Based on the incidence and registration of the Dutch Cancer Registry, our cohort consists of $20 \%$ of Dutch MCC patients compared to the SEER registry that currently covers $30 \%$ of the US population. ${ }^{16,42}$ One larger study by Field et al. was published although they comprised a significant longer timespan (40 years). ${ }^{21}$

We analysed a cohort with a patient population comparable to other cohorts concerning age, stage as well as comorbidities. ${ }^{13,20,21,23,28,38-40}$ Although the given primary treatments were heterogeneous, neither treatment regimens nor guidelines have altered much throughout the last decades. While immunotherapy is promising, only recently the first results have been published and none of the patients in our 
cohort received immunotherapy for MCC. ${ }^{7,13,43,44}$ Consequently, neither patient population nor improvement in therapies can sufficiently explain the difference in OS and MCC related death.

Several population-based series have been published. . $^{5,7,9,19,22,23}$ While the number of patients from such studies is enlarged significantly, detailed data on treatment or MCC related death is often lacking. Few studies have investigated disease specific endpoints and even fewer studies have performed a competing risk analysis, thus underestimating RFS or disease specific survival when performing a Cox regression. By performing a competing risk analysis a more accurate estimation is achieved by accounting for the fact that many patients eventually die of other causes. Field et al. performed such analysis however they did not evaluated the effect of postoperative RT. $^{21}$

Our study remains, however, a retrospective study with its shortcomings. Due to the heterogeneous treatment regimens evaluating postoperative RT is delicate. Ideally, our findings should be confirmed in prospective trials with investigating the possible effects of excision margins and therapy after recurrence.

Staging has proved to be an important prognostic factor in MCC patients previously. ${ }^{5,20}$ Unfortunately, missing PTS refrained us from staging all MCC patients according the American Joint Committee on Cancer (AJCC) Staging Manual 8th edition. However, we did try to incorporate the AJCC staging in our model by including PTS $>20 \mathrm{~mm}$ and nodal metastases which are major factors to determine stage in MCC patients and were previously used by Allen et al. for staging MCC patients. ${ }^{20}$ Multivariable analysis could only be performed for patients with a known PTS $(60 \%)$. A PTS $>20 \mathrm{~mm}$ showed a trend for a higher MCC related death. Other studies have shown a significant association, yet due to the missing PTS our dataset is likely to lack power. Furthermore, nodal metastases at diagnosis were a prognostic factor. As we see more missing PTS early in our study we believe this is because of the poor (non - electronic) documentation in earlier decades. Synchronously to PTS, data on tumour depth or lymph vascular invasion was missing. Smith et al. have shown their prognostic relevance. ${ }^{45}$ Though, they encountered comparable impaired data and analysis was ultimately performed on 160 of 375 patients.

Besides extent of the disease at diagnosis, the method used to determine staging is associated with survival. ${ }^{20,46}$ Implementation of SNP since 1995 in the two centers 
could have influenced staging our cohort. Nevertheless only 11 patients (3\%) without nodal involvement were included before 1995 and could have been misclassified. Another factor possibly influencing staging is the categorization of patients with local disease at diagnosis. Patients with nodal staging and recurrence within 3 months were categorized as recurrence, while patients without nodal staging and recurrence within 3 months were considered to have regional disease at diagnosis. It is possible patients with nodal staging were misdiagnosed, and nodal metastases were missed. However we believe this decision reflects common day practice. Moreover, only in a maximum of ten patients such misclassification could have taken place. Hence, we do not believe this compromised our results.

This retrospective evaluation was undertaken as a first step to centralize care for this rare cancer in the Netherlands. We believe this is increasingly important in this day and age, especially in light of recent developments, in the understanding and treatment of MCC and the introduction of effective immunotherapy for one. Moreover, considering the very heterogeneous treatment applied during the study period, it has lead us to formulate a Dutch Guideline (based on NCCN and German guidelines).

\section{Conclusion}

Although OS is poor, a relatively low MCC related death is seen. Heterogeneous treatment regimens were given but often consisted of surgery followed by LND and/or post-operative RT. Post-operative RT was associated with reduced recurrence of MCC. The male gender and loco regional metastasis at time of diagnosis were negative independent predictors of MCC related death. Due to the rarity and heterogeneous treatment of MCC patients, centralized care for MCC is desirable. 


\section{References}

1. Pectasides D, Pectasides M, Economopoulos T. Merkel cell cancer of the skin. Ann Oncol. 2006;17:1489-1495.

2. Poulsen M. Merkel-cell carcinoma of the skin. Lancet Oncol. 2004;5:593-599.

3. Schrama D, Ugurel S, Becker JC. Merkel cell carcinoma: recent insights and new treatment options. Curr Opin Oncol. 2012;24:141-149.

4. Wang TS, Byrne PJ, Jacobs LK, et al. Merkel cell carcinoma: update and review. Semin Cutan Med. Surg. 2011;30:48-56.

5. Harms KL, Healy MA, Nghiem P, et al. Analysis of prognostic factors from 9387 Merkel cell carcinoma cases forms the basis for the new 8th edition AJCC staging system. Ann Surg Oncol. 2016;23:3564-3571.

6. Poulsen M, Round C, Keller J, et al. Factors influencing relapse-free survival in Merkel cell carcinoma of the lower limb-a review of 60 cases. Int J Radiat Oncol Biol Phys. 2010;76: 393-397.

7. Lemos BD, Storer BE, lyer JG, et al. Pathologic nodal evaluation improves prognostic accuracy in Merkel cell carcinoma: analysis of 5823 cases as the basis of the first consensus staging system. JAm Acad Dermatol. 2010;63:751-761.

8. Medina-Franco H, Urist MM, Fiveash J, et al. Multimodality treatment of Merkel cell carcinoma: case series and literature review of 1024 cases. Ann Surg Oncol. 2001;8:204-208.

9. Mojica P, Smith D, Ellenhorn JD. Adjuvant radiation therapy is associated with improved survival in Merkel cell carcinoma of the skin. J Clin Oncol. 2007;25:10431047.

10. Strom T, Carr M, Zager JS, et al. Radiation therapy is associated with improved outcomes in Merkel cell carcinoma. Ann Surg Oncol. 2016;23:3572-3578.

11. Poulsen MG, Rischin D, Porter I, et al. Does chemotherapy improve survival in highrisk stage I and II Merkel cell carcinoma of the skin? Int J Radiat Oncol Biol Phys. 2006;64:114-119.

12. Tai PTH, Yu E, Winquist E, et al. Chemotherapy in neuroendocrine/ Merkel cell carcinoma of the skin: Case series and review of 204 cases. J Clin Oncol. 2000;18:2493-2499.

13. Bajetta E, Celio L, Platania M, et al. Single-institution series of earlystage Merkel cell carcinoma: long-term outcomes in 95 patients managed with surgery alone. Ann Surg Oncol. 2009;16:2985-2993.

14. Cassler NM, Merrill D, Bichakjian CK, et al. Merkel cell carcinoma therapeutic update. Curr Treat Options Oncol. 2016;17:36. 
15. Zaar O, Gillstedt M, Lindelof B, Wennberg-Larko AM, Paoli J. Merkelcell carcinoma incidence is increasing in Sweden.J Eur Acad DermatolVenereol. 2016;30:17081713.

16. Reichgelt BA, Visser O. Epidemiology and survival of Merkel cell carcinoma in the Netherlands. A population-based study of 808 casesin 1993-2007.Eur J Cancer. 2011;47:579-585.

17. Kukko H, Böhling T, Koljonen V, et al. Merkel cell carcinoma-Apopulation-based epidemiological study in Finland with a clinical series of 181 cases. Eur J Cancer. 2012;48:737-742.

18. Hodgson NC. Merkel cell carcinoma: changing incidence trends. J Surg Oncol. 2005;89:1-4.

19. Agelli M, Clegg LX. Epidemiology of primary Merkel cell carcinoma inthe United States.J Am Acad Dermatol. 2003;49:832-841.

20. Allen PJ, Bowne WB, Jaques DP, et al. Merkel cell carcinoma: prognosis and treatment of patients from a single institution. J Clin Oncol. 2005;23:2300-2309.

21. Fields RC, Busam KJ, Chou JF, et al. Five hundred patients with Merkel cell carcinoma evaluated at a single institution.AnnSurg.2011;254:465-475.

22. Albores-Saavedra J, Batich K, Chable-Montero F, et al. Merkel cell carcinoma demographics, morphology, and survival based on 3870cases: a population based study. J Cutan Pathol. 2010;37:20-27.

23. Iyer JG, Storer BE, Paulson KG, et al. Relationships among primary tumor size, number of involved nodes, and survival for 8044 cases of Merkel cell carcinoma. J Am Acad Dermatol. 2014;70:637-643.

24. Hui AC, Stillie AL, Seel M, Ainslie J. Merkel cell carcinoma: 27-yearexperience at the Peter MacCallum Cancer Centre. Int J Radiat Oncol Biol Phys. 2011;80:1430-1435.

25. Liang E, Brower JV, Rice SR, et al. Merkel cell carcinoma analysis of outcomes: a 30year experience. PLoS ONE. 2015;10:e0129476.

26. Jabbour J, Cumming R, Scolyer RA, et al. Merkel cell carcinoma: assessing the effect of wide local excision, lymph node dissection, and radiotherapy on recurrence and survival in early-stage disease - results from a review of 82 consecutive cases diagnosed between 1992 and2004. Ann Surg Oncol. 2007;14:1943-1952.

27. Tarantola TI, Vallow LA, Halyard MY, et al. Prognostic factors in Merkel cell carcinoma: analysis of 240 cases. J Am Acad Dermatol.2013;68:425-432.

28. Andea AA, Coit DG, Amin B, Busam KJ. Merkel cell carcinoma: histologic features and prognosis. Cancer. 2008;113:2549-2558.

29. Penberthy L. SEER Registries: Population-based Infrastructure to Support Cancer Research. Presented at the 6th Joint Meeting of the Board of Scientific Advisors and 
the National Cancer Advisory Board2015; Department of Health and Human Services \& Public Health Service \& National Institute of Health \& National Cancer Institute.

30. Nghiem PT, Bhatia S, Lipson EJ, et al. PD-1 blockade with pembrolizumab in advanced Merkel-cell carcinoma. N Engl J Med.2016;374:2542-2552.

31. Kaufman HL, Russell J, Hamid O, et al. Avelumab in patients with chemotherapyrefractory metastatic Merkel cell carcinoma: a multi-centre, single-group, open-label, phase 2 trial. Lancet Oncol.2016;17:1374-1385.

32. Bhatia S, Storer BE, lyer JG, et al. Adjuvant radiation therapy and chemotherapy in Merkel cell carcinoma: survival analyses of 6908cases from the national cancer data base. J Natl Cancer Inst.2016;108.

33. Lewis KG, Weinstock MA, Weaver AL, Otley CC. Adjuvant local irradiation for Merkel cell carcinoma. Arch Derm. 2006;142:693-700.

34. Harrington C, Kwan W. Outcomes of Merkel cell carcinoma treated with radiotherapy without radical surgical excision. Ann Surg Oncol.2014;21:34013405.

35. Smith VA, Camp ER, Lentsch EJ. Merkel cell carcinoma: identification of prognostic factors unique to tumors located in the head and neck based on analysis of SEER data. Laryngoscope. 2012;122:1283-1290.

36. Sridharan VS. Merkel cell carcinoma: a population analysis on survival. J Natl Compr Cancer Netw. 2016;14:1247-1257.

37. Scoggins CR, Ross MI, Reintgen DS, et al. Gender-related differences in outcome for melanoma patients. Ann Surg. 2006;243:693-698.discussion 8-700.

38. Schwartz JL, Wang TS, Hamilton TA, et al. Thin primary cutaneous melanomas: associated detection patterns, lesion characteristics, and patient characteristics. Cancer. 2002;95:1562-1568.

39. Oliveria SA, Christos PJ, Halpern AC, et al. Evaluation of factors associated with skin self-examination. Cancer Epidemiol Biomarkers Prev. 1999;8:971-978.

40. Joosse A, Collette S, Suciu S, et al. Sex is an independent prognostic indicator for survival and relapse/progression-free survival in metastasized stage III to IV melanoma: a pooled analysis of five European organisation for research and treatment of cancer randomized controlled trials.JClinOncol.2013;31:2337-2346.

41. Feldmeyer L, Hudgens CW, Lyons GR, et al. Density, distribution, and composition of immune infiltrates correlate with survival in Merkel cell carcinoma. Clin Cancer Res. 2016;22:5553-5563.

42. Ngo ST, Steyn FJ, McCombe PA. Gender differences in autoimmunedisease. Front Neuroendocrinol2014;35:347-369. 
Part I | Chapter 4

43. Smith FO, Yue B, Marzban SS, et al. Both tumor depth and diameter are predictive of sentinel lymph node status and survival in Merkel cell carcinoma. Cancer.

2015;121:3252-3260

44. Kaufman HL, Russell J, Hamid O, et al. Avelumab in patients with chemotherapyrefractory metastatic Merkel cell carcinoma: a multi-centre, single-group, open-label, phase 2 trial. Lancet Oncol.2016;17:1374-1385.

45. Smith FO, Yue B, Marzban SS, et al. Both tumor depth and diameter are predictive of sentinel lymph node status and survival in Merkel cell carcinoma. Cancer. 2015;121:3252-3260.

46. Moshiri AS, Nghiem P. Milestones in the staging, classification, and biology of Merkel cell carcinoma. J Natl Compr Canc Netw. 2014;12:1255-1262. 


\section{Supplementary Graphs S1: Univariable analyses}

\section{Relapse}
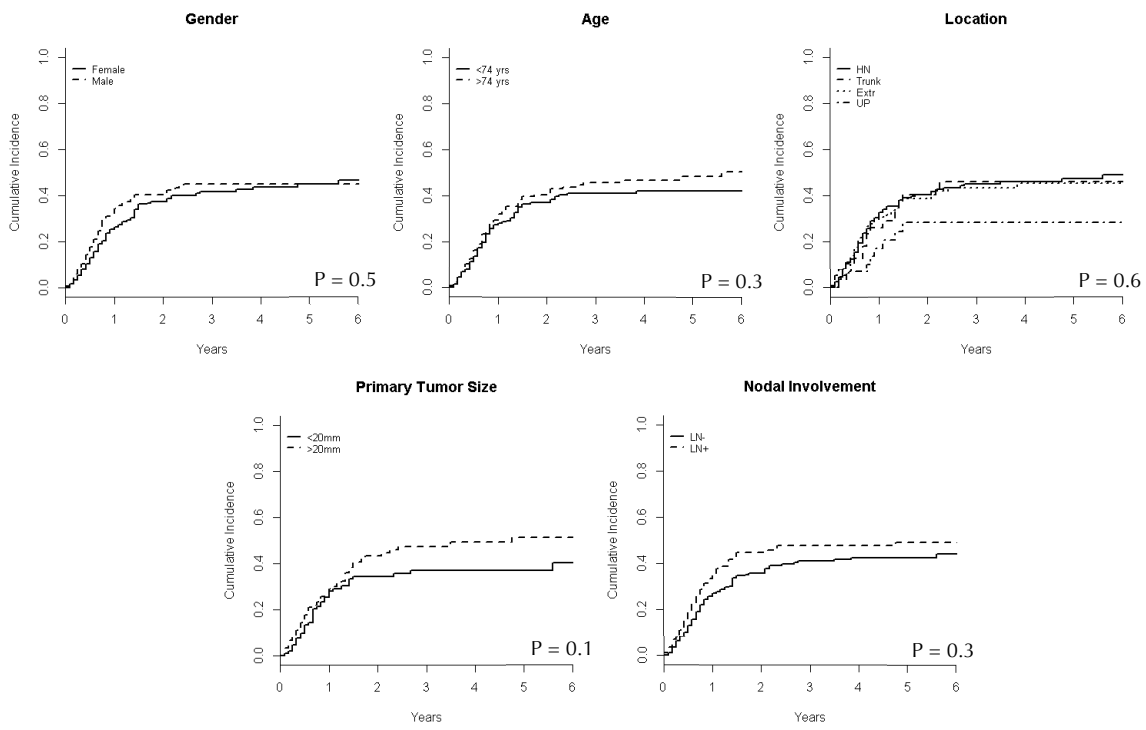

Overall Survival

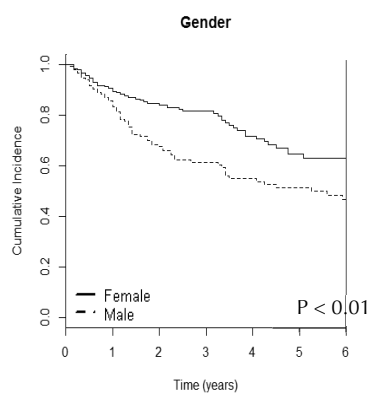

Primary Tumor Size

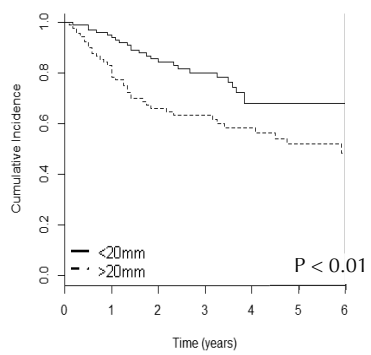

Age

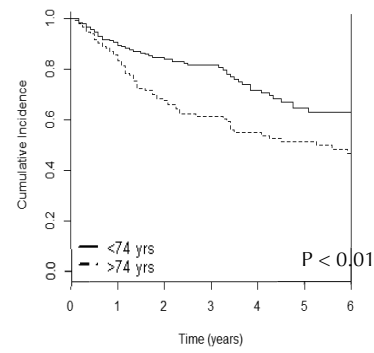

Nodal Involvement

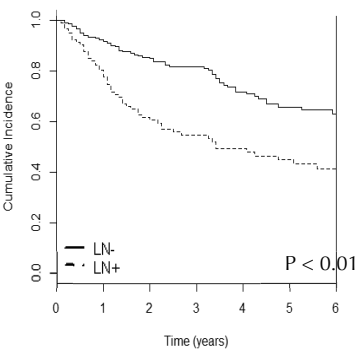

Location

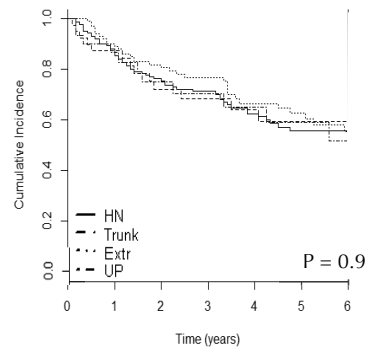

RT

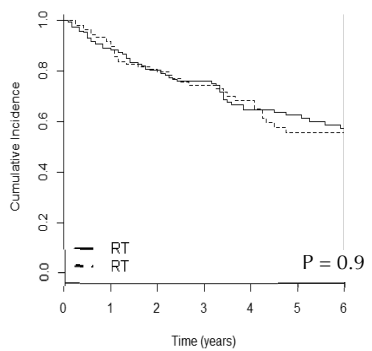


Part I | Chapter 4

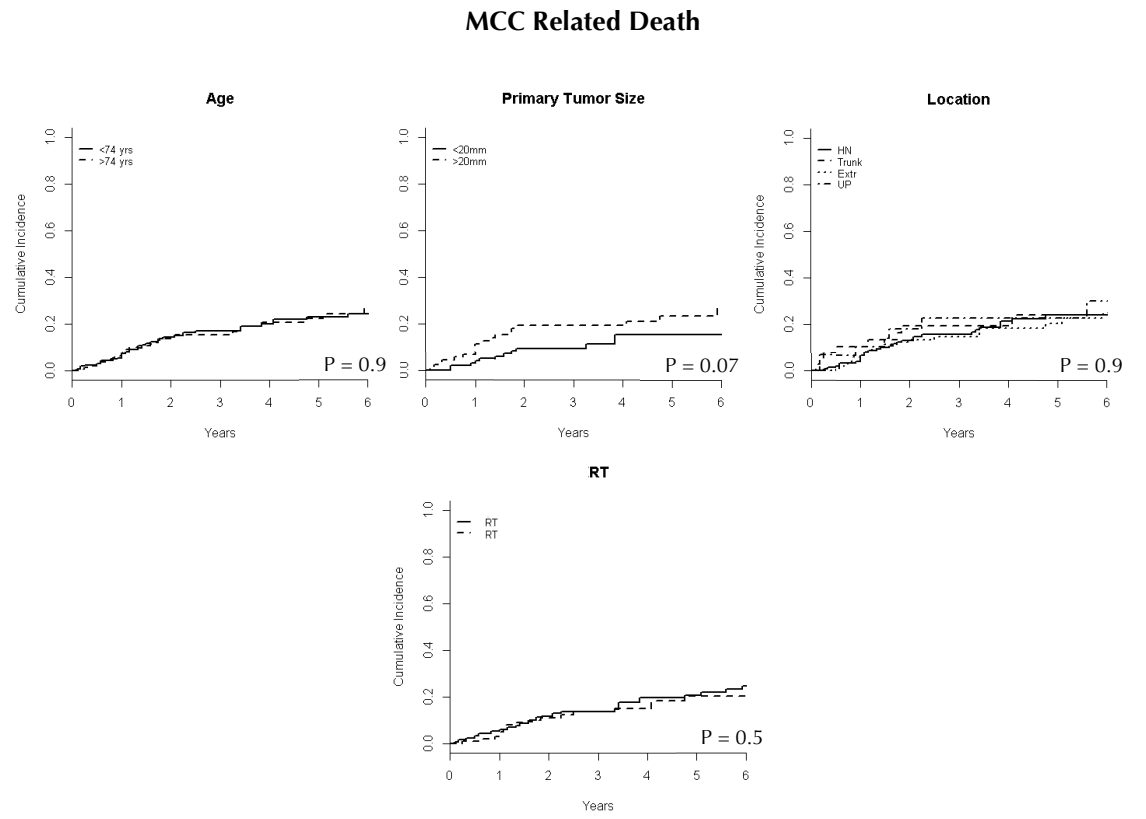





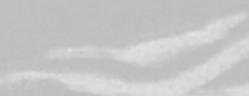

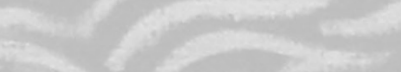

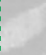

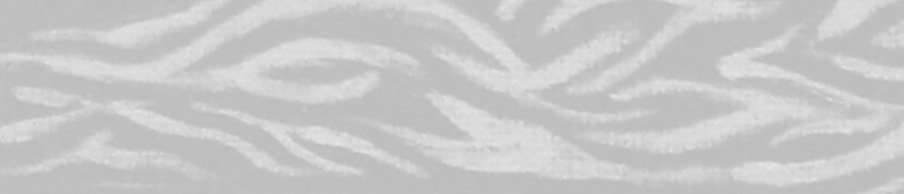




\section{Chapter 5}

The clinical utility of neuron-specific enolase serum levels as a biomarker for Merkel cell carcinoma

L.M. van Veenendaal, E. Bertolli, C.M. Korse, W.M.C. Klop, M.E.T. Tesselaar, A.C.J. van Akkooi

Annals of Surgical Oncology, 2020 


\section{Abstract}

\section{Background}

No adequate biomarker for Merkel cell carcinoma (MCC) has been identified. Serum neuron-specific enolase (NSE) has been tested and is commonly used as a biomarker for several other small cell malignancies. However, the role of NSE in MCC is still unclear.

\section{Aim}

To investigate the role of NSE as a biomarker in MCC.

\section{Patients and Methods}

A prospective cohort of MCC patients was analysed using Kaplan Meier curves with log rank test, ROC curves, Cox regression and mixed models. A separate evaluation was performed for patients treated with immunotherapy (IT).

\section{Results}

Eighty-four patients were included (47 males, median age 71 years, stages I\&II, III and IV MCC in respectively 39(46\%); 42(50\%) and 3(4\%) patients at time of diagnosis) with 565 NSE samples (median 15; IQR 12.6-22 ng/ml). Baseline NSE had no association with prognosis. NSE correlated with extent of disease $(P=0.01)$ and increased with $15 \mathrm{ng} / \mathrm{ml}$ per class (no tumour load, localized MCC, regional or distant metastases, respectively). NSE was able to detect progression (AUC 0.89). A NSE of $18.2 \mathrm{ng} / \mathrm{ml}$ was considered the most optimal level for clinical use (sensitivity 91\%, specificity 78\%, PPV 48\%, NPV 98\%). During IT ( $\mathrm{n}=23 ; 248$ NSE values) all complete responders $(n=10)$ had a normalized NSE $(<18.2 \mathrm{ng} / \mathrm{ml})$, all partial responders $\quad(n=5)$ had a decreasing NSE. In non-responders $(n=8)$ all NSE levels remained elevated.

\section{Conclusions}

NSE could be a valuable biomarker in MCC. NSE correlates with extent of disease; it is able to rule out progression and distinguishes responders from non-responders during IT. 


\section{Introduction}

Merkel cell carcinoma (MCC) is a neuroendocrine carcinoma of the skin with potential aggressive behaviour and an increasing global incidence..$^{1-3}$ Ultraviolet exposure is suggested to play a role in developing MCC. Additionally, a viral pathway induced by the Merkel cell Polyomavirus (MCPyV) is assumed to be involved in the pathophysiology of MCC. ${ }^{4}$ Furthermore, MCC occurs more frequently in immune compromised patients, e.g. HIV positive patients or patients using immunosuppressive agents. ${ }^{5}$

Most patients are diagnosed with early stage MCC which shows 5-year overall survival rate of approximately 40-62\%. ${ }^{6,7}$ Unfortunately, MCC has a high recurrence rate $(26-60 \%)$ and often occurs within 2 years. ${ }^{8}$ For patients with distant metastases 5 -year survival rates decrease dramatically to $13-27 \% .{ }^{5}$ Until recently, patients with distant metastases were treated with chemotherapy (CT) ensuring high response rates but often not durable. ${ }^{9,10}$ However, like in melanoma, recent findings in immunology and results from treating patients with immune checkpoint inhibitors have completely changed the treatment landscape and outlook for metastatic MCC patients showing promising results inducing durable partial or complete response rates. ${ }^{11-13}$

To date, no accurate biomarker for patients with MCC has been identified to predict prognosis or response after treatment. ${ }^{14}$ The high recurrence rate, the introduction of immunotherapy (IT) and subsequently the improved survival of patients with metastatic MCC emphasized the need for an accurate biomarker guiding treatment decisions.

The use of serum NSE as a biomarker is already accepted in small cell carcinomas such as small cell lung carcinoma and neuroblastoma. Several studies have showed its relevance in evaluating responsiveness to therapy and detecting early recurrences for these malignancies. ${ }^{15-19}$ Nevertheless data are scarce about the correlation of serum NSE and MCC, leaving the role of NSE in MCC still unclear. ${ }^{20-23}$ Neuronspecific enolase (NSE) is found in neuronal and neuroendocrine tissues. ${ }^{24,25}$ Using immunostaining techniques, NSE can be seen in the cytoplasm of several types of cells, including Merkel's cells of the skin under normal conditions. ${ }^{26-28}$ It is hypothesized that increased expression of NSE in tissue or serum levels is due to altered metabolism or turnover of these cells. Also, NSE has a relatively short biological half-life (approximately 24h). ${ }^{24}$ These features suggest that NSE could be a useful biomarker. We evaluated the role of NSE as a biomarker in MCC. 


\section{Patients and methods}

A consecutive cohort of patients with MCC treated in the Netherlands Cancer Institute from January 2016 until July 2018 was prospectively collected. The study was conducted according to institutional ethical guidelines. Patient's disease- and treatment characteristics were recorded. Registration of medical history and comorbidity aimed to identify diseases associated with elevated NSE values (small cell lung carcinoma, neuroblastoma and degenerative neurological disorders). At baseline and during follow-up (FU), patients were evaluated using different imaging modalities according to physicians' choice. Patients were staged according to the American Joint Committee on Cancer Staging System 2017. Patients were seen in our outpatient-clinic every 3 months in the first year, every 4 months in the second year, twice a year in the third year and once a year thereafter. At every visit NSE was determined. In case of a single elevated NSE regular FU was continued. In case of a second elevated NSE level radiological evaluation outside of protocol was performed to evaluate potential disease progression. Otherwise, patients still underwent routine FU imaging.

Blood was collected by using standard sampling tubes or tubes containing separating gel followed by centrifugation within one hour to avoid hemolysis. NSE levels were measured on the Cobas e 601 analyzer (Roche Diagnostics, Mannheim, Germany) using the electrochemiluminescence immunoassay (ECLIA) technique. The reference value in our laboratory was established at $<12.5 \mathrm{ng} / \mathrm{ml}$, the reference value recommended by the manufacturer was $12.95 \mathrm{ng} / \mathrm{ml}$.

- Patient categories

After collecting all data, patients were designated to categories according to the course of their disease: category A consisted of patients with localized MCC treated with primary surgery and no evidence of disease (NED) during FU. Patient category B consisted of patients with localized MCC treated with surgery and who experienced recurrence of MCC during FU. Patient category C consisted of patients who received systemic treatment, either as primary treatment or during FU. Per patient category, the development of NSE on patient level was visualized. 
- Extent of disease

For every NSE sample, extent of disease was registered. Extent of disease was divided into four classes: NED, presence of the primary tumour, in-transit or regional nodal metastases and distant (nodal) metastases. NSE in relation to extent of disease was analysed using a generalized linear mixed model (GLMM) with random effect, assuming random slopes and random intercept per patients to account for the repeated measures and variability per patient. In this analysis extend of disease was used as a predictor for NSE. The coefficient of the GLMM reflects the increase of NSE for every class. To analyse NSE and extent of disease more accurately a separate analysis was performed, in which samples obtained during systemic therapy were excluded.

\section{- Prognosis}

Baseline NSE was NSE level at time of diagnosis, whereas post-operative NSE was the first NSE level ( $<3$ months) determined after primary surgery. Baseline NSE and postoperative NSE (continuous and using the optimal cut-off) were evaluated in relation to recurrence free survival (RFS) and overall survival (OS). RFS and OS were calculated from date of diagnosis until progression and death. Since only one patient died of other causes than MCC, disease specific survival was not analysed. Prognostics analyses were performed using Kaplan Meier curves, log rank test (cut-off value) and Cox regression (continuous NSE). Patients that were lost to FU before reaching one of the endpoints were censored at their last time of FU. For Cox regression, associations were summarized by calculating hazard ratios (HR) and corresponding 95\% confidence intervals $(\mathrm{Cl})$.

\section{- Progression and response}

For every NSE sample, progression or response was registered. Progression could be the development of new lesions or progression of existing lesions. Local progression entailed the primary tumour or in-transit metastases (a cutaneous or subcutaneous metastasis between the primary location and tumour draining nodal basin) and could be confirmed clinically. Nodal and distant progression entailed (distant) lymph nodes or organs, which had to be confirmed by histopathology or by radiological examination. Response was defined as no evidence of disease after surgery or a radiological response of at least $>30 \%$ after systemic therapy determined by a 
radiologist. In case of no radiological or clinical evaluation combined with no evidence for progression or response on the next evaluation, previous disease status was continued. To evaluate NSE in detecting progression an AUC was calculated according to Obuchowski, which takes clustered data into account and handles the variability in repeated measures. ${ }^{29}$ To evaluate NSE in detecting response, a reversed AUC was calculated. Both absolute NSE value and change of NSE in ng/ml (between two samples) were analysed.

- Optimal cut-off value

Sensitivity, specificity, positive predictive value (PPV), negative predictive value (NPV), likelihood ratio (LR) and accuracy were compared for several cut-off values of NSE based on 1) the reference value by the manufacturer $(12.95 \mathrm{ng} / \mathrm{ml}), 2)$ the institutional reference $(12.5 \mathrm{ng} / \mathrm{ml}), 3)$ the Youdens' Index calculated from the receiver operating characteristic curve (ROC), which aims to identify an optimal threshold considering both sensitivity and specificity, 4) 95\% sensitivity.

- NSE evaluation during IT

Using the optimal cut-off value, NSE was analysed in detail in patients during IT by differentiating several types of response and progression: progressive disease (PD), mixed response $(\mathrm{MR})$, partial response (PR), complete response (CR) or stable disease (SD). PR was defined as $>30 \%$ decrease of existing lesions. CR was defined as a radiological disappearance of all lesions. MR occurred when MCC lesions showed response as well as progression. PD occurred in case of new lesions or an increase $>20 \%$ of existing lesions. SD was considered the absence of either response or progression.

- Statistical analysis

Data were summarized using standard descriptive statistics: frequency and percentage for categorical variables and mean with standard deviation (SD) or median with interquartile range (IQR) and range for continuous variables according to their distribution. All calculated $P$ values were two-sided and $P$ values less than .05 were considered statistically significant. All analyses were performed using SPSS software version 24 (SPSS Inc., Chicago, IL) or R version 3.4.1, an open source statistical software (www.r-project.org). 


\section{Results}

Of 100 eligible patients, 84 patients had at least one NSE value. Baseline characteristics are summarized in Table 1 . The cohort consisted of 47 male (56\%) and 37 female (44\%) patients with a median age of 71 years. Most patients $(n=32$, $38 \%$ ) had a primary tumour on the extremities and were diagnosed a stage III MCC $(\mathrm{n}=42 ; 50 \%) .605$ Blood samples were obtained, of which 36 samples (6\%) were not evaluable due to haemolysis. The median number of blood samples per patient was 4 (IQR 2-7) with a median value of $15.0 \mathrm{ng} / \mathrm{ml}$ (IQR 12.6-22.0).

Table 1: Baseline characteristics

\begin{tabular}{|c|c|c|c|c|c|c|}
\hline \multirow[b]{2}{*}{ Characteristic } & \multicolumn{2}{|c|}{ Eligible cohort $(N=100)$} & \multicolumn{2}{|c|}{ NSE $(\mathrm{N}=84)$} & \multicolumn{2}{|c|}{ non-NSE $(N=16)$} \\
\hline & median & $\%$ or IQR & n or median & $\%$ or IQR & n or median & $\%$ or IQR \\
\hline \multicolumn{7}{|l|}{ Gender: } \\
\hline Male & 55 & $55 \%$ & 47 & $56 \%$ & 8 & $50 \%$ \\
\hline Female & 45 & $45 \%$ & 37 & $44 \%$ & 8 & $50 \%$ \\
\hline Age: & 72 years & $(65-79)$ & 71 years & $(64-78)$ & 73 years & $(67-80)$ \\
\hline \multicolumn{7}{|l|}{ Topography: } \\
\hline H\&N & 32 & $32 \%$ & 24 & $29 \%$ & 8 & $50 \%$ \\
\hline Trunk & 17 & $17 \%$ & 13 & $15 \%$ & 4 & $25 \%$ \\
\hline Extr & 33 & $33 \%$ & 32 & $38 \%$ & 1 & $6 \%$ \\
\hline UP & 18 & $18 \%$ & 15 & $18 \%$ & 3 & $19 \%$ \\
\hline \multicolumn{7}{|l|}{ Stage:* } \\
\hline $\mathbf{I}$ & 27 & $27 \%$ & 20 & $23 \%$ & 7 & $44 \%$ \\
\hline II & 10 & $10 \%$ & 10 & $12 \%$ & - & - \\
\hline Ila & 6 & $6 \%$ & 6 & $7 \%$ & - & - \\
\hline Illb & 4 & $4 \%$ & 3 & $4 \%$ & 1 & $6 \%$ \\
\hline III & 2 & $2 \%$ & 2 & $2 \%$ & - & - \\
\hline IIIa & 12 & $12 \%$ & 9 & $11 \%$ & 3 & $19 \%$ \\
\hline IIIb & 35 & $35 \%$ & 31 & $37 \%$ & 4 & $25 \%$ \\
\hline VI & 4 & $4 \%$ & 3 & $4 \%$ & 1 & $6 \%$ \\
\hline NSE related comorbidity: & 0 & 0 & 0 & 0 & 0 & 0 \\
\hline
\end{tabular}


Treatment and FU characteristics are summarized in Table 2. Median FU was 20 months (IQR7-28). During FU 36 patients (42\%) relapsed with a median RFS of 13 months (IQR 8-20). At the end of FU 52 patients (62\%) had NED, ten patients $(12 \%)$ died, one patient died due to other causes, while nine patients succumbed to MCC. A total of 22 patients (26\%) were alive with disease at end of FU.

Table 2: Treatment and follow-up NSE cohort

\begin{tabular}{|c|c|c|}
\hline & $\begin{array}{r}\text { n or } \\
\text { median }\end{array}$ & $\begin{array}{c}\% \text { or } \\
\text { IQR }\end{array}$ \\
\hline \multicolumn{3}{|l|}{ NSE: } \\
\hline Total samples & 605 & - \\
\hline Haemolytic samples & 36 & $6 \%$ \\
\hline NSE level (ng/ml) & 15 & $\begin{array}{r}12.6- \\
22\end{array}$ \\
\hline Samples per patient & 4 & $2-7$ \\
\hline Baseline samples & 36 & $43 \%$ \\
\hline Post-operative samples & 24 & $29 \%$ \\
\hline \multicolumn{3}{|l|}{ Primary Treatment: } \\
\hline Surgery (+/- RT) & 70 & $83 \%$ \\
\hline $\mathrm{CT}+$ surgery & 1 & $1 \%$ \\
\hline IT & 5 & $6 \%$ \\
\hline RT & 1 & $1 \%$ \\
\hline ILP & 1 & $1 \%$ \\
\hline Declined treatment & 4 & $5 \%$ \\
\hline \multicolumn{3}{|c|}{ Treatment during follow up: } \\
\hline Surgery (+/- RT) & 16 & $33 \%$ \\
\hline Surgery + adj CT & 2 & $4 \%$ \\
\hline CRT & 2 & $4 \%$ \\
\hline ILP & 2 & $4 \%$ \\
\hline $\mathrm{CT}$ & 10 & $20 \%$ \\
\hline IT & 18 & $36 \%$ \\
\hline
\end{tabular}

\begin{tabular}{lrr}
$\begin{array}{l}\text { (continuation) } \\
\begin{array}{r}\text { nedian } \\
\text { Follow-up (months): }\end{array}\end{array}$ & $\begin{array}{r}\% \text { or } \\
\text { IQR }\end{array}$ \\
\hline TFD to 1st sample & 9 & $6-17$ \\
\hline TFD to recurrence & 13 & $6-20$ \\
\hline Total folluw-up time & 20 & $7-28$ \\
\hline Clinical outcome: & & \\
\hline Recurrence & 36 & $42 \%$ \\
\hline Vital Status at end of follow up: & & \\
\hline NED & 52 & $62 \%$ \\
\hline AWD & 22 & $26 \%$ \\
\hline MCC-D & 9 & $11 \%$ \\
\hline DOC & 1 & $1 \%$ \\
\hline Patient category: & & \\
\hline Category A & 32 & $38 \%$ \\
\hline Category B & 22 & $26 \%$ \\
\hline Category C & 30 & $36 \%$ \\
\hline
\end{tabular}

NSE: neuron specific enolase; $R T$ : radiation therapy; adj: adjuvant; $R T$ : radiation therapy; $C T$ : chemotherapy; ILP: Isolated limb perfusion; IT: immunotherapy; TFD: time from diagnosis; NED: no evidence of disease; AWD: alive with disease; MCC-D: MCC related death; DOC: death due to other causes 
Patients were divided into three categories according to the course of their disease as mentioned in the Patients \& Methods section. Category A (localized MCC treated with primary surgery) consisted of 32 patients (38\%), category B (localized MCC treated with surgery and experienced recurrence) consisted of 22 patients (26\%) and category $\mathrm{C}$ (patients that underwent systemic treatment) consisted of 30 patients (36\%). During FU, the development of NSE varied considerably per patient category (Graph 1-4).
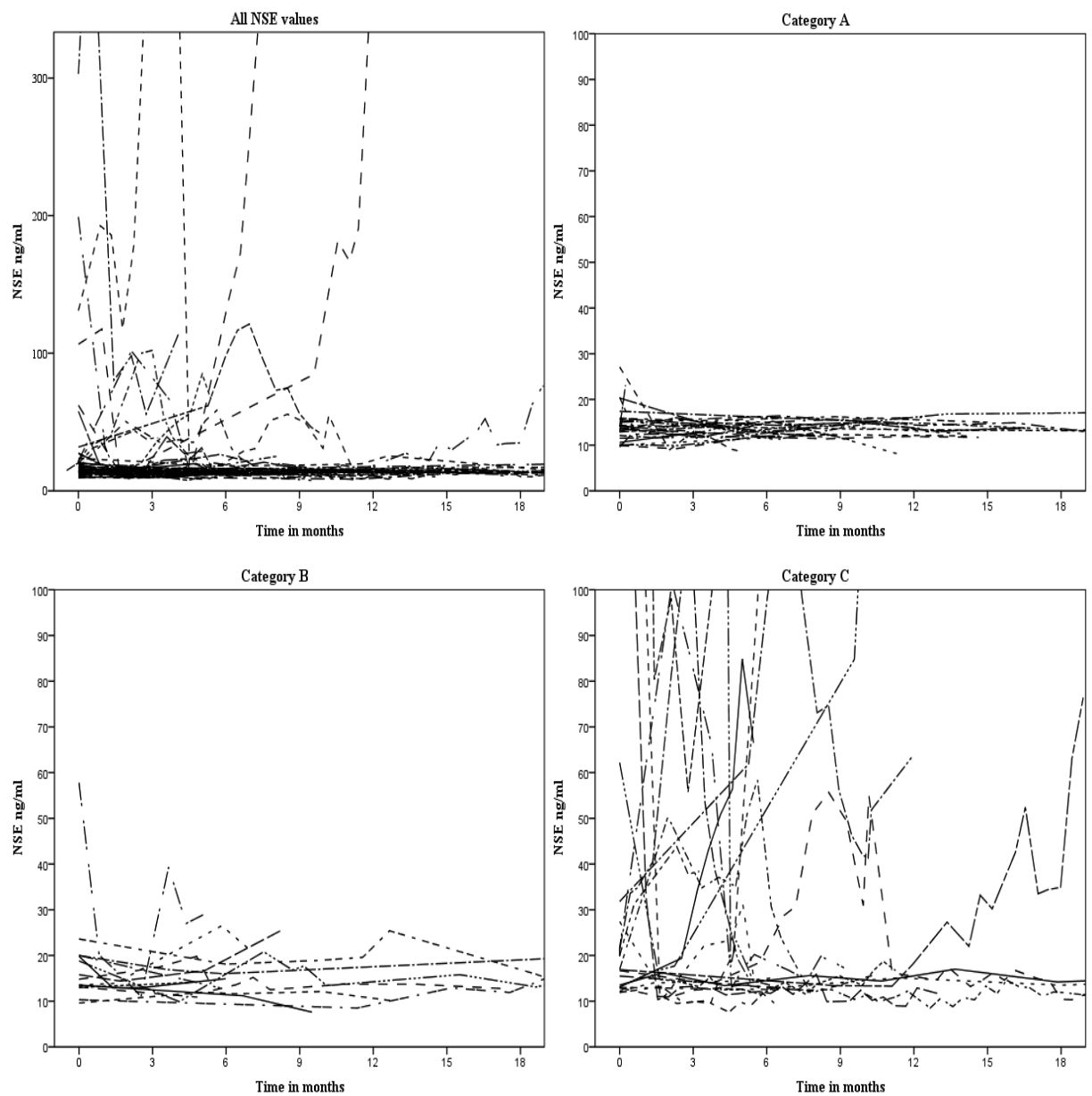

Graphs 1-4: NSE development per patient category 
NSE was evaluated in relation to extent of disease, RFS, OS, progression and response (an overview is presented in Table 3).

Whether NSE corresponded with extent of disease was analysed in a GLMM for which 565 NSE samples were available. In our cohort, NSE increased with 15.0 $\mathrm{ng} / \mathrm{ml}$ with every class of extent of disease being: no evidence of disease, (recurrence) primary tumour, in-transit and/or regional metastases or distant metastases. The increase of NSE for every class proved statistically significant $(P=0.01)$. Excluding blood samples retrieved under systemic treatment, NSE $(\mathrm{n}=225)$ showed an even stronger correlation with an increase of NSE of $16.1 \mathrm{ng} / \mathrm{ml}$ for every class $(\mathrm{P}=0.01)$

Furthermore, NSE and its association with RFS and OS was evaluated. Thirthy-six baseline NSE samples (43\%) and 24 post-operative NSE samples (31\%) were available. Both baseline and postoperative NSE (continuous and using a cut-off of $18.2 \mathrm{ng} / \mathrm{ml}$ ) were not associated with RFS or OS. Additional survival curves are found in Supplement 1.

Also, the ability of NSE (565 samples) to detect progression and response was analyzed. First, an AUC for progression (0.89) was calculated and for response a reversed AUC (0.67). An AUC for change in NSE (ng/ml between 2 samples) was calculated as well. These AUCs showed comparable results; an AUC of 0.75 and 0.61 for progression and response, respectively. To assess the performance of NSE in detecting progression on patient level, sensitivity, specificity, PPV, NPV, LR and accuracy for different cut-off values was calculated (Table 4).

The cut-off values based on the institutional reference value and manufacturers' index were $12.5 \mathrm{ng} / \mathrm{ml}$ and $12.95 \mathrm{ng} / \mathrm{ml}$, respectively. Sensitivity for progression and NPV was high, however with a low specificity and low PPV. A cut-off value based on a sensitivity of $95 \%$ led to a NSE of $16.4 \mathrm{ng} / \mathrm{ml}$. As expected, a higher specificity was seen. Based on the Youdens' index (optimal threshold based on the AUC) a NSE value of $18.2 \mathrm{ng} / \mathrm{ml}$ was calculated, leading to a sensitivity of $91 \%$, specificity of $78 \%$, PPV of $48 \%$ and NPV of $98 \%$, with an accuracy of $81 \%$. A threshold of 18.2 $\mathrm{ng} / \mathrm{ml}$ was considered the most appropriate to determine the clinical utility of NSE in the cohort. This threshold still holds a high NPV with considerable improvement in accuracy compared to the other cut-off values. 
Table 3: Statistical analyses

\begin{tabular}{|c|c|c|c|c|}
\hline Relation & NSE & samples & effect & $P$ or $95 \% \mathrm{Cl}$ \\
\hline \multirow[t]{4}{*}{ RFS } & Baseline (continuous) & 36 & HR 1.0 & 0.09 \\
\hline & Baseline (>18.2 ng/ml) & 36 & $\mathrm{n} / \mathrm{a}^{*}$ & 0.3 \\
\hline & Post-operative (continuous) & 24 & HR 0.9 & 0.6 \\
\hline & Post-operative (>18.2 ng/ml) & 24 & $\mathrm{n} / \mathrm{a}^{*}$ & 0.6 \\
\hline \multirow[t]{4}{*}{ OS } & Baseline (continuous) & 36 & HR 0.9 & 0.5 \\
\hline & Baseline $(>18.2 \mathrm{ng} / \mathrm{ml})$ & 36 & $\mathrm{n} / \mathrm{a}^{*}$ & 0.3 \\
\hline & Post-operative (continuous) & 24 & HR 0.9 & 0.4 \\
\hline & Post-operative (>18.2 ng/ml) & 24 & $\mathrm{n} / \mathrm{a}^{*}$ & 0.6 \\
\hline \multirow[t]{2}{*}{ Extent of disease } & NSE & 565 & $15 \mathrm{ng} / \mathrm{ml}$ & 0.01 \\
\hline & NSE (excl. syst. treatm.)** & 225 & $16,1 \mathrm{ng} / \mathrm{ml}$ & 0.01 \\
\hline \multirow[t]{2}{*}{ Progression } & NSE & 565 & AUC 0.89 & $0.84-0.95$ \\
\hline & Change in NSE & 400 & $\mathrm{~A} \cup \mathrm{C} 0.75$ & $0.69-0.80$ \\
\hline \multirow[t]{2}{*}{ Response } & NSE & 565 & rev. AUC 0.67 & $0.55-0.81$ \\
\hline & Change in NSE & 400 & rev. AUC 0.61 & $0.57-0.65$ \\
\hline
\end{tabular}

*survival curves in supplementary; **excluding samples taken during systemic treatment; RFS: recurrence free survival, OS: overall surviva; rev: reversed; AUC: area under the curve

Table 4: Diagnostic performance per NSE threshold value for progression

\begin{tabular}{|c|c|c|c|c|c|c|}
\hline & sensitivity & specificity & PPV & NPV & LR & accuracy \\
\hline $12.5 \mathrm{ng} / \mathrm{ml}^{*}$ & $99 \%$ & $28 \%$ & $22 \%$ & $99 \%$ & 1.97 & $40 \%$ \\
\hline $12.95 \mathrm{ng} / \mathrm{ml}^{* *}$ & $98 \%$ & $34 \%$ & $24 \%$ & $99 \%$ & 1.48 & $45 \%$ \\
\hline $16.4 \mathrm{ng} / \mathrm{ml}^{* * *}$ & $95 \%$ & $70 \%$ & $40 \%$ & $99 \%$ & 3.13 & $74 \%$ \\
\hline $18.2 \mathrm{ng} / \mathrm{ml}^{* * * *}$ & $91 \%$ & $78 \%$ & $48 \%$ & $98 \%$ & 4.22 & $81 \%$ \\
\hline
\end{tabular}

\section{- Clinical utility of NSE}

Using the cut-off value of $18.2 \mathrm{ng} / \mathrm{ml}$, the use of NSE to predict progression was evaluated in the different patient categories. In patient category A, all 32 patients (117 samples) had NSE $<18.2 \mathrm{ng} / \mathrm{ml}$ corresponding with the absence of recurrence. 
Patient category B consisted of 22 patients and 132 NSE samples. In three patients, NSE was not elevated while patients progressed. Nevertheless, NSE showed an increase of almost $200 \%$ in one patient. This patient was NED after wide local excision of the primary tumour and recurred in a regional lymph node after 6 months during which NSE increased to 17.0 coming from $8.6 \mathrm{ng} / \mathrm{ml}$. After lymph node dissection, NSE normalized and patients remained NED during 2,5 years of FU. In two other patients, (regional) recurrence was detected clinically and involved little tumour volume. The first patient had a locally advanced MCC at the lower extremities for which she received Isolated Limb Perfusion (ILP) with a clinically near complete response. PET/CT confirmed local residual disease without other metastases. NSE values ranged between 9.6-12.1 ng/ml after ILP and during residual disease. The residual lesion was resected after which she remained NED with NSE $<18.2 \mathrm{ng} / \mathrm{ml}$ during 2 years of FU.

The second patient had a MCC on the extremities for which local excision and a sentinel node procedure was performed (pT1N1M0 stage IIB) and post-operative radiotherapy (RT) was advised but declined. During FU she developed histopathologically confirmed in-transit metastases. She underwent ILP with good response. Afterwards, her NSE varied between 12.9-14.9 ng.ml. However, after 3 months nodules were visible and any diagnostic or therapeutic interventions were declined, including NSE evaluation.

Conversely, one patient had a single NSE level of $20.0 \mathrm{ng} / \mathrm{ml}$ during FU without progression (confirmed by a negative PET/CT), hereafter NSE normalized and remained low without progression during FU.

Patient category C consisted of 24 patients and 316 samples. In only one patient, NSE was not elevated during progressive disease. This patient had a temporal MCC $(2.6 \mathrm{~cm})$ and PET/CT suggested nodal metastases, which were treated with local excision, lymph node dissection (3 positive lymph nodes) and RT (66 Gy in 33 fractions). During routine FU, a PET/CT was made which showed a solitaire metastasis in L2. NSE was $16.2 \mathrm{ng} / \mathrm{ml}$ coming from $13.5 \mathrm{ng} / \mathrm{ml}$. After chemoradiation therapy NSE decreased to $13.0 \mathrm{ng} / \mathrm{ml}$. However, after 12 months NSE increased to $17.0 \mathrm{ng} / \mathrm{ml}$ and multiple ossal metastases were seen. IT followed with a PR and 12/32 NSE levels were $<18.2 \mathrm{ng} / \mathrm{ml}$ during FU.

In 17 patients NSE was $>18.2 \mathrm{ng} / \mathrm{ml}$ without simultaneous radiological progression (67 samples) of which eight patients (21 samples; 31\%), progressed within 12 weeks after the sample was obtained. One patient had a single elevated NSE which normalized without progression during FU. In another patient NSE (2 samples) 
remained elevated after start of IT but no response evaluation was performed due to progression of preexistent lung fibrosis which ultimately led to death. In the remaining seven patients (43 samples; 64\%) NSE was elevated but showed a decreasing trend after start of systemic therapy (15 samples; $22 \%$ ) or was obtained during PR or MR on IT (29 samples; 44\%).

\section{- NSE during IT}

NSE was analysed in detail in patients during IT. In 23 patients NSE during IT (248 samples) could be evaluated in relation to treatment response. Fifteen patients showed a response on IT. All complete responders $(n=10)$ had a normalized NSE $(<18.2 \mathrm{ng} / \mathrm{ml})$ at first radiological evaluation. Patients with a PR or MR $(\mathrm{n}=5)$ showed a substantial decrease in NSE, of which two patients showed transiently normalizing NSE values. Corresponding to their clinical course during IT, 30 of 55 NSE samples remained elevated for these patients. The remaining eight patients were non responders (NR), showing elevated and increased NSE levels after start of IT. Noteworthy, one of our patients with a CR showed progression at first response evaluation, although after a switch from a PDL1-inhibitor to a PD1-inhibitor a CR and normalization of NSE was seen. An overview of the NSE development per patient during IT is presented in Table 5 and Graph 5-7. 
Part I | Chapter 5

Table 5: Overview NSE during IT per patient

\begin{tabular}{|c|c|c|c|c|c|c|c|c|}
\hline$\#$ & Response & $\begin{array}{l}\text { NSE start } \\
\text { IT (ng/ml) }\end{array}$ & $\begin{array}{c}\text { samples } \\
\text { (n) }\end{array}$ & $\begin{array}{r}\text { NSE } \\
<18.2 \\
\mathrm{ng} / \mathrm{ml}(\mathrm{n}) \\
\end{array}$ & $\begin{array}{r}\text { NSE } \\
>18.2 \\
\mathrm{ng} / \mathrm{ml}(\mathrm{n}) \\
\end{array}$ & $\begin{array}{r}\text { highest } \\
\text { NSE } \\
(\mathrm{ng} / \mathrm{ml})\end{array}$ & $\begin{array}{r}\text { lowest } \\
\text { NSE } \\
(\mathrm{ng} / \mathrm{ml}) \\
\end{array}$ & $\begin{array}{r}\text { duration } \\
\text { IT } \\
\text { (weeks) }\end{array}$ \\
\hline 1 & PR & 17,1 & 22 & 5 & 17 & 55,2 & 13,8 & 75 \\
\hline 2 & CR & 16,2 & 20 & 20 & 0 & 17,5 & 10,3 & 50 \\
\hline 3 & PR & 98,2 & 12 & 0 & 12 & 121,2 & 41,3 & 22 \\
\hline 4 & NR & 181,6 & 4 & 0 & 4 & 400,2 & 166,8 & 6 \\
\hline 5 & CR & 58,6 & 27 & 24 & first 3 & 58,6 & 8,5 & 62 \\
\hline 6 & NR & 22 & 11 & 0 & 11 & 76,4 & 22 & 20 \\
\hline 7 & NR & 173,1 & 2 & 0 & 2 & 419,2 & 173,1 & 4 \\
\hline 8 & CR & 15,2 & 14 & 14 & 0 & 15,5 & 11,5 & 39 \\
\hline 9 & NR & 37,8 & 2 & 0 & 2 & 38,4 & 37,8 & 2 \\
\hline 10 & CR & 32,6 & 24 & 24 & first 1 & 32,6 & 8,9 & 52 \\
\hline 11 & NR & 19,4 & 6 & 0 & 6 & 84,9 & 34,1 & 13 \\
\hline 12 & PR & 66 & 10 & 6 & 4 & 66 & 13 & 20 \\
\hline 13 & PR & 11 & 6 & 6 & 0 & 13,5 & 11 & 28 \\
\hline 14 & $\mathrm{CR}^{*}$ & 21,4 & 15 & 4 & 11 & 102,1 & 14,3 & 33 \\
\hline 15 & CR & 27,4 & 3 & 2 & first 1 & 27,4 & 14,6 & 12 \\
\hline 16 & NR & 192,7 & 8 & 0 & 8 & 544,8 & 117,4 & 14 \\
\hline 17 & CR & 11,9 & 10 & 10 & 0 & 13,2 & 7,6 & 20 \\
\hline 18 & NR & 446 & 3 & 0 & 3 & 446 & 173 & 12 \\
\hline 19 & NR & 214 & 5 & 0 & 5 & 317 & 204 & 10 \\
\hline 20 & PR & 20,8 & 12 & 4 & 8 & 37 & 14,8 & 20 \\
\hline 21 & CR & 129 & 10 & 5 & 5 & 185 & 10 & 20 \\
\hline 22 & CR & 127,1 & 10 & 6 & 4 & 127,01 & 9,1 & 20 \\
\hline 23 & MR & 76,2 & 12 & 0 & 12 & 76,2 & 31,8 & 16 \\
\hline
\end{tabular}




\section{Complete responders}

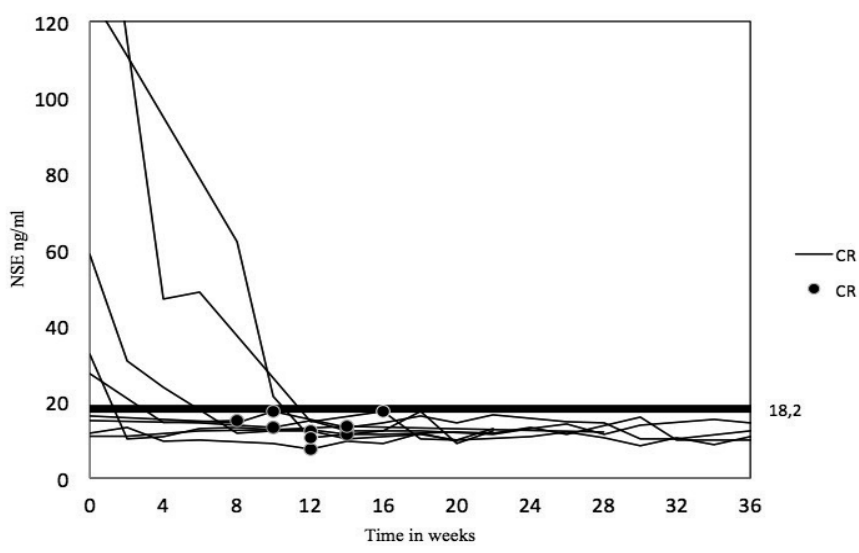

Mixed and partial responders

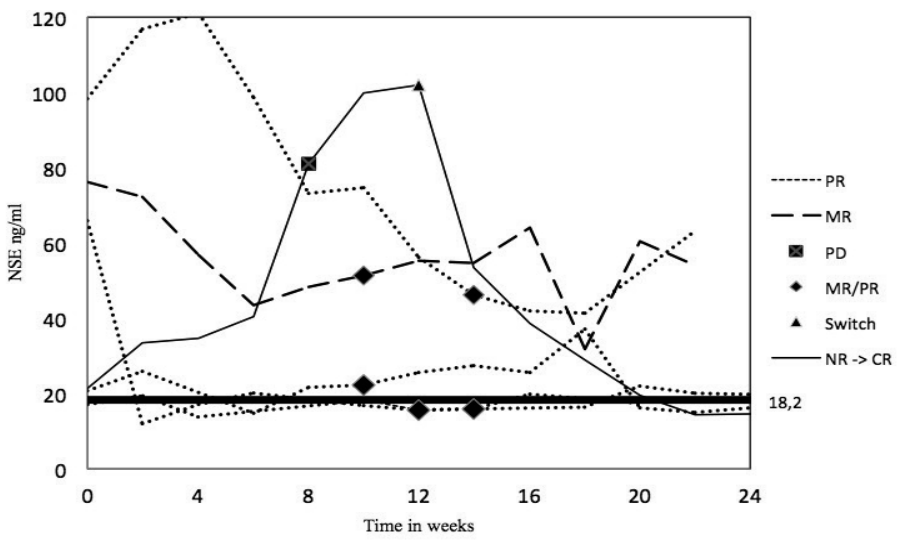

Non responders

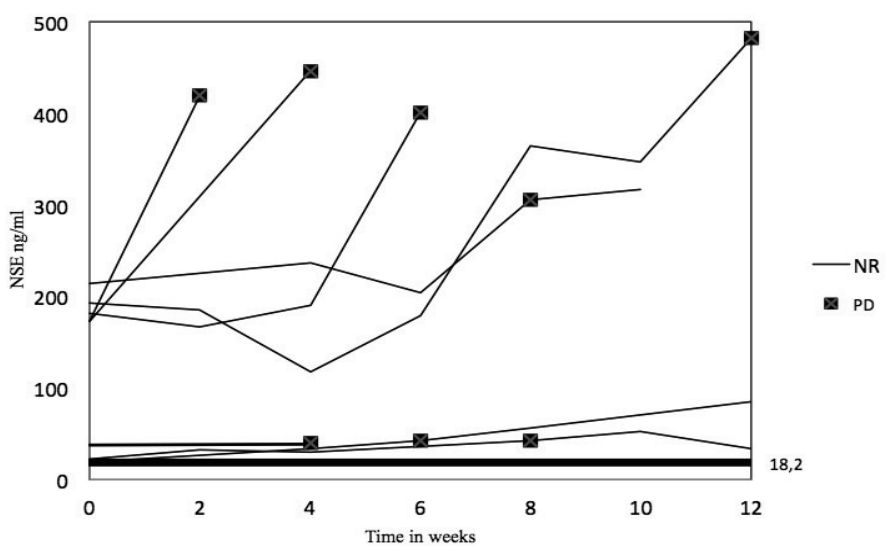

Graphs 5-6: NSE development during IT 


\section{Discussion}

This study is the largest study to evaluate NSE in patients with MCC. Our study showed a correlation between NSE and extent of disease $(P=0.01)$ and demonstrated a promising performance in detecting progressive disease with a NPV of $98 \%$. During IT, normalizing NSE distinguished complete responders from nonresponders.

One other study by Gaiser et al. investigated serum NSE and its association with recurrence, survival and tumour burden. ${ }^{21}$ In contrast to our findings, NSE was not considered an effective biomarker. However, NSE was either categorically interpreted (within normal limits $<17.0 \mathrm{ng} / \mathrm{ml}$, above normal limits and high $>24.3$ $\mathrm{ng} / \mathrm{ml}$ ) or the maximum or distribution of NSE was used. This statistical approach of NSE might not reflect the biological relationship of NSE with MCC and could be the reason a correlation was missed.

The outstanding performance of NSE in detecting progression was expressed by an AUC of 0.89. Several threshold values were analysed. For clinical use, we considered NPV the most relevant parameter, bearing in mind the potential aggressive behaviour of MCC. The threshold of $18.2 \mathrm{ng} / \mathrm{ml}$ calculated with the Youdens' index resulted in a NPV of 98\%, comparable to the NPV of lower threshold values but with considerably less false positive evaluations. Using this threshold, progressive disease was not recognized in four patients (5\%). In one patient NSE doubled (but remained $<18.2 \mathrm{ng} / \mathrm{ml}$ ), whereas in two patients metastases were of little volume and clinically detected. Only in one patient (1\%) progression would have been missed using NSE alone.

The relatively low PPV was due to elevated NSE levels without simultaneous radiological progression in 18 patients (21\%). In two patients NSE spontaneously decreased, whereas one patient died before response evaluation could take place. Another seven patients showed a significant decrease after start of systemic treatment and had a PR corresponding to elevated yet decreasing NSE values. In eight patients progression occurred within 12 weeks after the first elevated NSE value, suggesting that NSE could be useful for early detection as well. This however, should be thoroughly investigated with a more strict study protocol. Nevertheless even with a relatively low PPV, the clinical use of NSE still holds relevant information for physicians treating MCC patients. With novel options for treatment of metastatic 
MCC such as pembrolizumab and avelumab, finding biomarkers to detect recurrences and monitor response on IT could aid treatment decisions and improve outcome. In our IT cohort, NSE was able to distinguish responders from nonresponders. In case of a CR normalization of NSE levels within 3 months were seen, whereas NR showed increasing NSE values on IT.

Besides NSE, Chromogranin A (CgA), antibodies against MCPyV-encoded oncoproteins and circulating tumour cells (CTC) has been proposed as a potential promising biomarker. ${ }^{21,30-32}$ Despite the use of CgA in monitoring neuroendocrine tumours, CgA has not been able to distinguish MCC patients with or without evidence of disease. ${ }^{21}$ CTCs have been studied as a potential biomarker, but have not been adequately evaluated during $\mathrm{FU}$ or in relation to response during systemic therapy. There has been increasing interest in MiRNAs as a potential biomarker in several malignancies. ${ }^{33}$ Although one study showed an association between MiRNAs, MCPyV postitive tumours and clinical outcome, its exact implication needs further investigation. ${ }^{34,35}$ More recently, antibodies against MCPyV-encoded oncoproteins have shown encouraging results, comparable to NSE in our study. ${ }^{31}$ However depending on the population, approximately $50 \%$ of patients have a non-MCPyV associated MCC and would not produce antibodies against MCPyV-encoded oncoproteins, hampering the use of these antibodies as a biomarker. ${ }^{36}$

Several limitations of our study should be taken into consideration. Despite our large cohort and vast amount of blood samples, few baseline and post-operative NSE values were available because we are a tertiary referral center and patients often are presented after initial diagnosis and primary treatment. The lacking prognostic value of NSE could be explained by the deficient baseline samples. Nevertheless, it is possible that NSE serum level is primarily a reflection of tumour burden. Thus a prognostic association might be lacking, even when more baseline samples could be obtained.

Some of the eligible MCC patients did not undergo NSE evaluation. This resulted in a relatively low representation of MCC patients with a primary tumour located in the head and neck region. However, to our knowledge there has not been any indication that NSE levels differ within a certain malignancy due to its primary location.

Despite a uniform FU protocol, time interval varied between samples and frequency of NSE samples varied, possibly leading to an unbalanced cohort of repeated measures. To correct for this unbalance, we developed a generalized mixed model 
with random effects evaluating NSE and extent of disease. Likewise, we calculated a clustered AUC, taking into account repeated measures. Furthermore, despite our promising results, the cut-off value used in the analyses was only determined and evaluated within our cohort. To ensure adequate implementation of NSE as a biomarker in MCC, we strongly recommend and encourage validation in external populations.

\section{Conclusion}

NSE could be a valuable biomarker in patients with MCC. NSE corresponds with extent of disease and is able to rule out progression. Furthermore, NSE distinguishes responders from non-responders during IT. 


\section{References}

1. Youlden DR, Soyer HP, Youl PH et al. Incidence and survival for Merkel cell carcinoma in Queensland, Australia, 1993-2010. JAMA Dermatol 2014; 150: 864872.

2. Reichgelt BA, Visser O. Epidemiology and survival of Merkel cell carcinoma in the Netherlands. A population-based study of 808 cases in 1993-2007. Eur J Cancer 2011;47:579-585.

3. Harms KL, Healy MA, Nghiem P et al. Analysis of Prognostic Factors from 9387 Merkel Cell Carcinoma Cases Forms the Basis for the New 8th Edition AJCC Staging System. Ann Surg Oncol 2016;23:3564-3571.

4. Harms PW, Harms KL, Moore PS et al.International Workshop on Merkel Cell Carcinoma Research (IWMCC) Working Group. The biology and treatment of Merkel cell carcinoma: current understanding and research priorities. Nat Rev Clin Oncol. 2018 Dec;15(12):763-776.

5. Becker JC, Stang A, Hausen AZ et al. Epidemiology, biology and therapy of Merkel cell carcinoma: conclusions from the EU project IMMOMEC. Cancer Immunol Immunother 2018;67:341-351.

6. Fields RC, Busam KJ, Chou JF et al. Five Hundred Patients With Merkel Cell Carcinoma Evaluated at a Single Institution. Annals of Surgery 2011;254:465-475.

7. van Veenendaal LM, van Akkooi ACJ, Verhoef $C$ et al. Merkel cell carcinoma: Clinical outcome and prognostic factors in 351 patients. J Surg Oncol 2018;117:1768-1775.

8. Fields RC, Busam KJ, Chou JF et al. Recurrence and survival in patients undergoing sentinel lymph node biopsy for merkel cell carcinoma: analysis of 153 patients from a single institution. Ann Surg Oncol 2011;18:2529-2537.

9. Poulsen MG, Rischin D, Porter I et al. Does chemotherapy improve survival in highrisk stage I and II Merkel cell carcinoma of the skin? Int J Radiat Oncol Biol Phys 2006;64:114-119.

10. Tai PT, Yu E, Winquist E et al. Chemotherapy in neuroendocrine/Merkel cell carcinoma of the skin: case series and review of 204 cases. Journal of Clinical Oncology 2000;18:2493-2499.

11. Kaufman HL, Russell J, Hamid O et al. Avelumab in patients with chemotherapyrefractory metastatic Merkel cell carcinoma: a multicentre, single-group, open-label, phase 2 trial. Lancet Oncol 2016;17:1374-1385. 
12. Nghiem PT, Bhatia S, Lipson EJ et al. PD-1 Blockade with Pembrolizumab in Advanced Merkel-Cell Carcinoma. New England Journal of Medicine 2016;374:2542-2552.

13. Kaufman HL, Russell JS, Hamid O et al. Updated efficacy of avelumab in patients with previously treated metastatic Merkel cell carcinoma after $>/=1$ year of follow-up: JAVELIN Merkel 200, a phase 2 clinical trial. J Immunother Cancer 2018;6:7.

14. Schadendorf D, Lebbé C, zur Hausen A et al. Merkel cell carcinoma: Epidemiology, prognosis, therapy and unmet medical needs. European Journal of Cancer 2017;71:53-69.

15. Harmsma M, Schutte B, Ramaekers FC. Serum markers in small cell lung cancer: opportunities for improvement. Biochim Biophys Acta 2013;1836:255-272.

16. Burghuber OC, Worofka B, Schernthaner G et al. Serum neuron-specific enolase is a useful tumor marker for small cell lung cancer. Cancer 1990;65:1386-1390.

17. Fizazi K, Cojean I, Pignon JP et al. Normal serum neuron specific enolase (NSE) value after the first cycle of chemotherapy: an early predictor of complete response and survival in patients with small cell lung carcinoma. Cancer 1998;82:1049-1055.

18. van Adrichem RC, Kamp K, Vandamme T et al. Serum neuron-specific enolase level is an independent predictor of overall survival in patients with gastroenteropancreatic neuroendocrine tumors. Ann Oncol 2016;27:746-747.

19. Riley RD, Heney D, Jones DR et al. A systematic review of molecular and biological tumor markers in neuroblastoma. Clin Cancer Res 2004;10:4-12.

20. Plowman PN. Serum marker for Merkel cell tumour. Clin Radiol 1989;40:542.

21. Gaiser MR, Daily K, Hoffmann J et al. Evaluating blood levels of neuron specific enolase, chromogranin A, and circulating tumor cells as Merkel cell carcinoma biomarkers. Oncotarget 2015;6:26472-26482.

22. Nobels FRE, Kwekkeboom DJ, Coopmans W et al. Chromogranin A as serum marker for neuroendocrine neoplasia: Comparison with neuron-specific enolase and the $\alpha$ subunit of glycoprotein hormones. 1997;82:2622-2628.

23. Giannone L, Johnson DH, Grosh WW et al. Serum neuron-specific enolase in metastatic Merkel cell tumors. Med Pediatr Oncol 1985;13:357-362.

24. Isgrò MA, Bottoni P, Scatena R. Neuron-Specific Enolase as a Biomarker: Biochemical and Clinical Aspects. Adv Exp Med Biol. 2015;867:125-143.

25. Kaiser E, Kuzmits R, Pregant $P$ et al. Clinical biochemistry of neuron specific enolase. Clin Chim Acta 1989;183:13-31.

26. Kirkham N, Isaacson P. Merkel cell carcinoma: a report of three cases with neuronespecific enolase activity. Histopathology 1983;7:251-259. 
27. Gould VE, Moll R, Moll I et al. Neuroendocrine (Merkel) cells of the skin: hyperplasias, dysplasias, and neoplasms. Lab Invest 1985;52:334-353.

28. Gu J, Polak JM, Van Noorden S et al. Immunostaining of neuron-specific enolase as a diagnostic tool for Merkel cell tumors. Cancer 1983;52:1039-1043.

29. Obuchowski NA. Nonparametric analysis of clustered ROC curve data. Biometrics 1997; 53:567-578.

30. Nobels FR, Kwekkeboom DJ, Coopmans W et al. Chromogranin A as serum marker for neuroendocrine neoplasia: comparison with neuron-specific enolase and the alpha-subunit of glycoprotein hormones. J Clin Endocrinol Metab 1997;82:26222628.

31. Paulson KG, Lewis CW, Redman MW et al. Viral oncoprotein antibodies as a marker for recurrence of Merkel cell carcinoma: A prospective validation study. Cancer 2017;123:1464-1474.

32. Riethdorf S, Hildebrandt L, Heinzerling L et al. Detection and Characterization of Circulating Tumor Cells in Patients with Merkel Cell Carcinoma. Clin Chem 2019;65:462-472.

33. Wang $\mathrm{H}$, Peng $\mathrm{R}$, Wang J et al. Circulating microRNAs as potential cancer biomarkers: the advantage and disadvantage. Clin Epigenetics. 2018;10:59.

34. Xie H, Lee L, Caramuta $\mathrm{S}$, et al. MicroRNA expression patterns related to merkel cell polyomavirus infection in human merkel cell carcinoma. J Invest Dermatol. 2014;134(2):507-517.

35. Konstatinell A, Coucheron DH, Sveinbjørnsson B et al. MicroRNAs as Potential Biomarkers in Merkel Cell Carcinoma. Int J Mol Sci. 2018;19(7).

36. Erovic I, Erovic BM. Merkel cell carcinoma: the past, the present, and the future. J Skin Cancer. 2013;64:92-93. 
Part I | Chapter 5

\section{Supplementary Graphs S1: Survival curves}
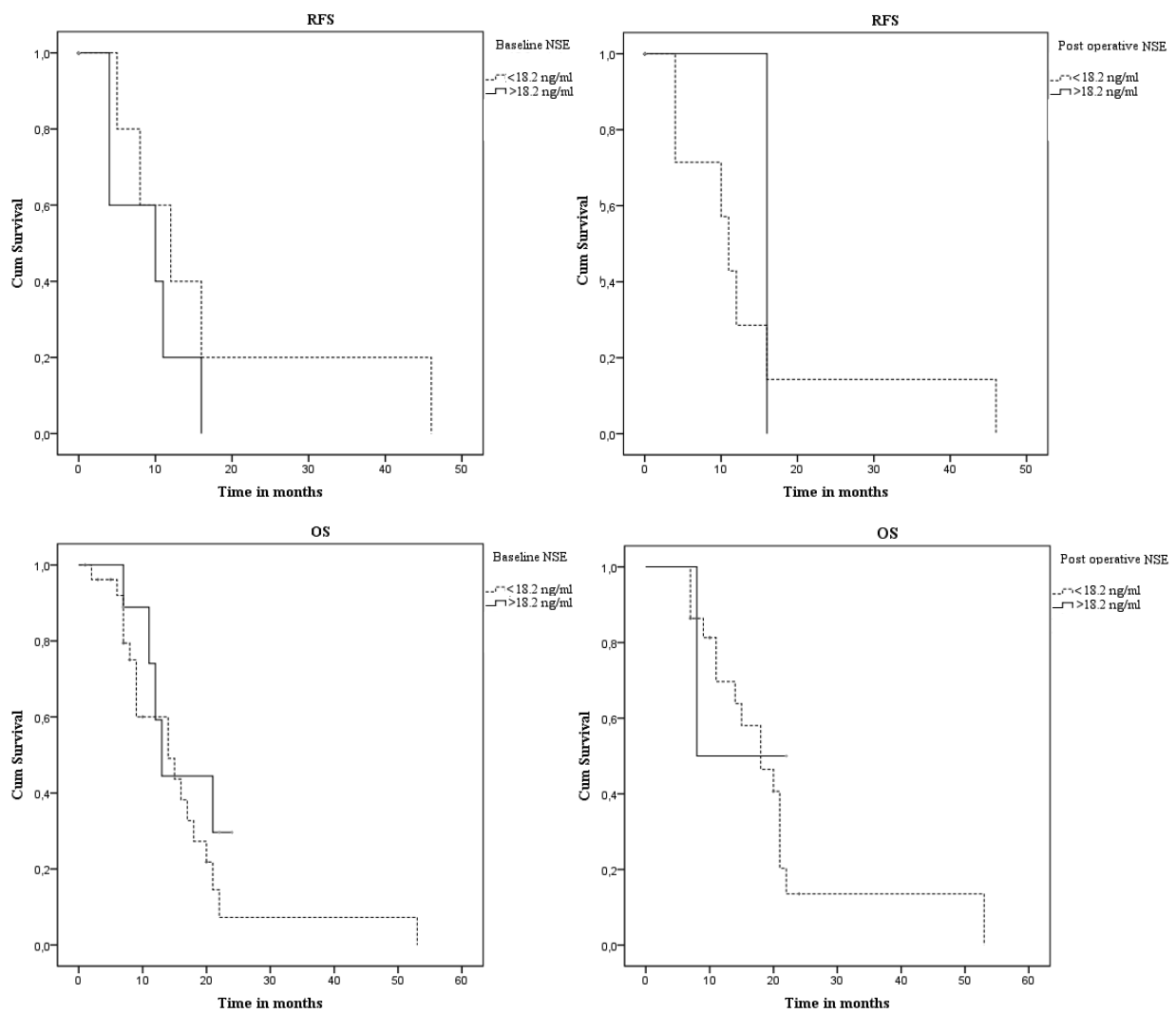



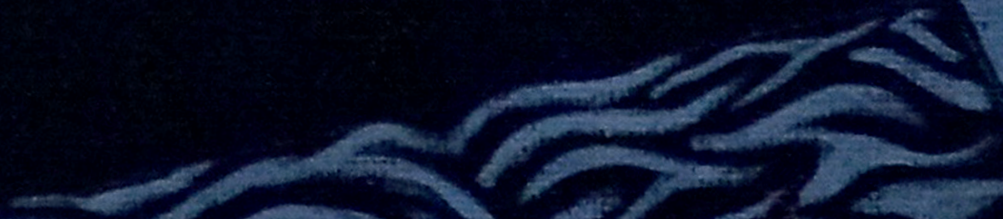

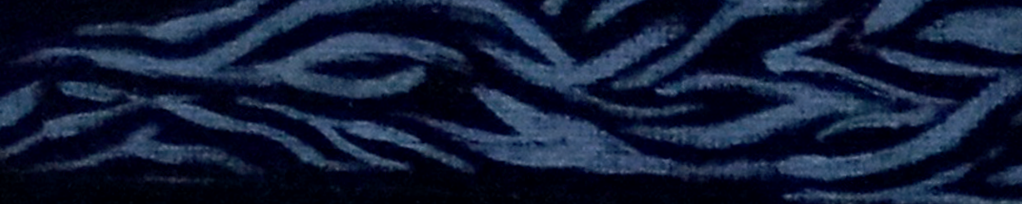




\title{
Part II
}

\author{
Treatment of Patients with \\ Neuroendocrine Neoplasia
}

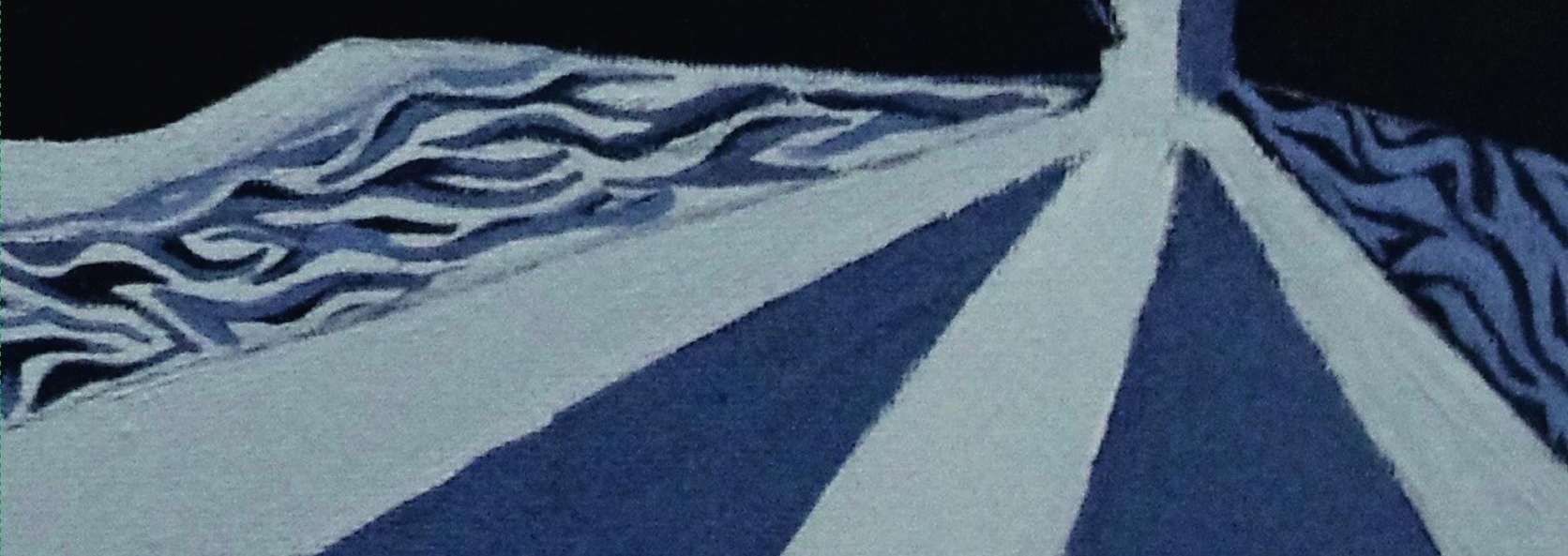




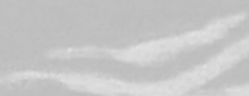

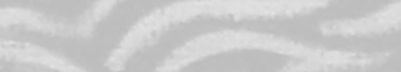

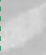

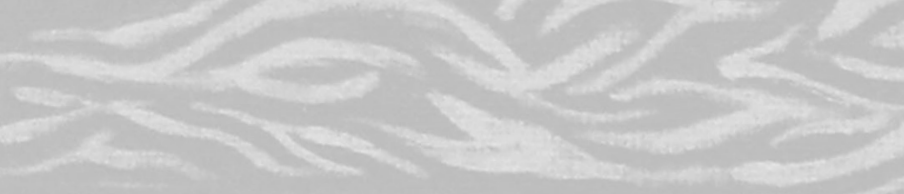




\section{Chapter 6}

Clinical benefit of systemic treatment in patients with advanced pancreatic and gastro-intestinal neuroendocrine tumours according to ESMO-MCBS and ASCO framework

L. de Hosson, L.M. van Veenendaal, Y. Schuller, W. Zandee, W. de Herder, M.

Tesselaar, H.J. Klumpen, A. Walenkamp

Annals of Oncology, 2017 


\section{Abstract}

\section{Background}

Assessment of clinical benefit of systemic treatments of rare diseases including gastroenteropancreatic neuroendocrine tumours (GEP-NET) is challenging. Recently several tools have been developed to grade the clinical benefit of cancer drugs. The European Society for Medical Oncology (ESMO) has developed the ESMO Magnitude of Clinical Benefit Scale (ESMO-MCBS). The American Society of Clinical Oncology (ASCO) has developed and revised the ASCO framework consisting of the Net Health Benefit (NHB) score juxtaposed against the costs of the treatment.

\section{Aim}

In this review, we graded systemic treatments for patients with GEP-NETs with both frameworks.

\section{Method}

The electronic databases (PubMed and EMBASE) were searched for papers reporting comparative trials, conducted in adult patients with GEP-NETs in the English language. Papers were assessed according to the ESMO-MCBS and the NHB part of the ASCO revised Framework (NHB-ASCO-F) by four independent assessors, and discrepancies were discussed.

\section{Results}

The search yielded 32 trials of which 6 were eligible for grading with the ESMOMCBS resulting in scores of 2 or 3 . Eight trials were eligible for grading with the NHB-ASCO-F, resulting in scores between 37.6 and 57.4. Trials that were not primary assessable by the tools were analysed separately. Consensus between assessors was reached in $68 \%$ of trials with the ESMO-MCBS and in $23 \%$ of trials with the NHB-ASCO-F.

\section{Conclusion}

The currently used systemic treatments for patients with GEP-NETs had low scores according to the NHB-ASCO-F and none could be graded as meaningful clinical beneficial according to the ESMO-MCBS. Despite the low incidence, the heterogeneous patient population and relatively long natural course of NET, future studies on new treatment modalities should aim for high clinical benefit outcomes. 


\section{Introduction}

Neuroendocrine tumours are rare malignancies with an incidence of 3.5/100 000 per year and a prevalence of 21.6/100 000 in the last decade. ${ }^{1}$ Median survival time is 77 months in patients with regional and 24 months in patients with distant metastatic pancreatic neuroendocrine tumour (pNET) and 105 months and 56 months in case of regional and distant metastatic intestinal NET disease, respectively. ${ }^{2}$

Gastroenteropancreatic neuroendocrine tumours (GEP-NET) patients are frequently metastasized at the time of initial diagnosis. Surgery is the only curative treatment. Non-curative systemic treatment options include somatostatin analogues (SSA), chemotherapy, targeted agents and peptide receptor radionuclide therapy (PRRT) with variable, limited success. ${ }^{3-12}$

Ideally, in clinical trials investigating novel drugs, the primary aim should be to study end points such as overall survival (OS), quality of life (QoL) and treatment toxicity. However, for trials investigating new agents in patients with GEP-NET, survival analysis can be challenging as the prolonged (natural) course of the disease often results in trials, allowing crossover towards the experimental arm, which influences OS. Crossover to the experimental arm or to second-line therapies has impact on OS data. ${ }^{13}$ Therefore, progression-free survival (PFS) was recommended as a feasible and relevant primary end point for both phase II and III trials in GEP-NET, by the expert consensus report of the National Cancer Institute Neuroendocrine Tumour Clinical Trials Planning Meeting. ${ }^{13}$

Recently, among others, European Society for Medical Oncology (ESMO) has developed a validated and reproducible tool to assess the magnitude of clinical benefit for drugs for solid tumours, the ESMO-MCBS. ${ }^{14}$ The American Society of Clinical Oncology (ASCO) developed the ASCO framework for solid tumours and haematological malignancies consisting of the Net Health Benefit (NHB) score juxtaposed against the costs of the treatment. ${ }^{15,16}$ Both tools have been applied in several solid malignancies. ${ }^{14-18}$ However, the ASCO framework was assessed only in a few studies. Treatments for GEP-NET are not yet evaluated using the tools. In this review, we therefore investigated the value of current systemic antitumor treatments for patients with GEP-NETs and their eligibility for grading with both ESMO-MCBS and NHB-ASCO-F. 
Part II | Chapter 6

\section{Method}

- Search strategy

For detailed description of methods, see supplementary Appendix S1. In brief, trials were searched between 1 August 2015 and 31st January 2016, in the databases PubMed and EMBASE. The articles that were found were screened using the title and abstract to select trials published in the English language and comparing systemic treatment modalities for the treatment of GEP-NET in humans. Furthermore, the reference lists of national and international guidelines that included trials and conference abstracts were reviewed for additional relevant articles.

- Selection criteria for trials

Comparative trials investigating systemic antitumor treatment for well-differentiated patients with GEP-NETs were analysed. Studies in patients with grade 3 neuroendocrine neoplasms were not included. Criteria for assessment are summarized in supplementary Table S1. Trials were selected if at least $50 \%$ of participants were diagnosed with GEP-NET, and the other participants had a NET of unknown or other origin. Abstracts were not included.

- ESMO-MCBS and the NHB-ASCO-F

ESMO-MCBS grades, in the non-curative setting, range from 1 to 5 , with grades 4 and 5 representing meaningful clinical benefit. ${ }^{14}$ The NHB-ASCO-F ranges from -20 to 180 , with a higher score representing a better score, and no cut-off value was provided to define clinical benefit. ${ }^{15,16,18}$ All relevant comparative trials were assessed according to both tools. Relevant trials that did not meet all criteria as mentioned in supplementary Table S1, were assessed separately, based on the available data.

- Assessment of trials

Four members from all Dutch European Neuroendocrine Tumour Society (ENETS) centres of excellence independently scored the trials according to the ESMO-MCBS and the NHB-ASCO-F. After obtaining scores of these assessors, we noticed a wide variation in results. During a consensus meeting, additional agreements were defined. Next, trials were assessed again according to the additional agreements. The 
number of trials to which the same score was awarded between three or four assessors was registered after each of the two scoring sessions. Two phase III trials were published after the consensus meeting and were assessed according our agreements. ${ }^{8,12}$ Therefore, an additional assessment session was not necessary for these trials.

\section{Results}

- Trial selection and characterization

The primary search strategy yielded 1942 potentially relevant papers, of which 1676 remained when duplicates were discarded (supplementary Figure S1). After screening the title and abstract, 223 papers were preselected evaluating systemic antitumor treatment for patients with GEP-NETs.

Thirty-nine papers of which 35 comparative trials in patients with GEP-NETs were described were selected for further analysis. Nine trials fulfilled all criteria as summarized in supplementary Table S1, and were assessable according to the ESMO-MCBS and the NHB-ASCO-F (Table 1). ${ }^{3-12}$ Only one trial, the RADIANT-4, described in its protocol that crossover was not allowed. ${ }^{10,11}$ None of the trials investigated adjuvant treatment in GEP-NET or had a curative intent.

The other 26 comparative trials, investigating systemic treatment in patients with GEP-NETs, did not meet all criteria for assessment with both tools (supplementary Table S1), or evaluated participants of whom less than 50\% had a GEP-NET. Main difficulties for assessment with the tools were summarized in supplementary Table S2. As currently used national and international guidelines are based on these trials, we analysed them separately with the available data (supplementary Table S3 and Appendix S2).

- Assessment according to the ESMO-MCBS

Six trials were assessable with the ESMO-MCBS. The calculated score ranged from 2 to 3 corresponding with a low level of clinical benefit. RADIANT-3 and RADIANT-4 analysed everolimus versus placebo in pNET and non-functional advanced NET, respectively. ${ }^{9-11}$ Application of ESMO-MCBS for RADIANT-3 and RADIANT-4 
resulted in a preliminary score of 3 reflecting a longer PFS. A hazard ratio $(H R) \leq 0.65$ with a lower limit of the $95 \%$ confidence interval for PFS determined the preliminary score. No significant difference in important adverse events as compared with the placebo control arm was detected. QoL of everolimus in PNET patients was reported in a recent abstract that described a single-arm phase IV study, carried out in patients who started with everolimus. ${ }^{19}$ After 6 months, no improvement in QoL was detected. Because this was a single-arm study and the ESMO-MCBS could only be applied to comparative outcome studies, the preliminary score of the RADIANT-3 will not be downgraded, despite lack of improvement of QoL, when these data are published in a full report. QoL of RADIANT-4 trial was reported in a post hoc analysis and recently published. ${ }^{11}$ No improvement of QoL by everolimus versus the control arm was demonstrated. Therefore, the preliminary score of the RADIANT-4 was downgraded to a final score 2 . The NETTER-1 trial investigated $177 \mathrm{Lu}$-dotatate with octreotide LAR versus octreotide LAR alone in patients with metastatic midgut NET. $^{8}$ The calculated ESMO-MCBS score was 3, which implies a longer PFS in the intervention group as compared with control arm. Furthermore, there was no significant difference in important adverse events as compared with the control arm and no QoL data were documented.

In the CLARINET trial, patients with a GEP-NET or patients with NET of unknown origin were treated with lanreotide in the intervention arm versus placebo in the control arm. ${ }^{3}$ This trial showed an improvement in PFS. Data of QoL did not show an improvement in QoL for the intervention group, resulting in a score of 2. In the PROMID trial, patients with a metastasized midgut NET were randomized between octreotide LAR and placebo. ${ }^{7}$ Further enrolment was stopped after inclusion of 85 patients instead of the planned 162 patients, because of observed positive effects of octreotide LAR on tumour growth and a slow recruitment rate. This trial resulted in an ESMO-MCBS score of 2. The trial reported by Raymond et al. analysed sunitinib versus placebo in patients with progressive, advanced pNET. ${ }^{6}$ Application of ESMOMCBS resulted in a score of 2. An improvement in PFS was shown. Because reported QoL data did not show improvement, the preliminary score of 3 had to be downgraded one point. This trial had an early closure after randomization of 171 patients, as observed and recommended by the safety monitoring board due to more serious adverse events and a higher frequency of death in the placebo group as well as a difference in PFS favouring sunitinib. The trial analysing SSA and interferon versus SSA and bevacizumab, in advanced NET patients with progression or other poor prognostic features, showed no significant difference in its primary end point 
PFS. ${ }^{12}$ The RADIANT-2 analysed everolimus with long acting octreotide in advanced NET tumour patients associated with carcinoid syndrome. ${ }^{5}$ The PFS with an HR of 0.77 (0.59-1.00) did not show a statistically significant clinical benefit. The trial analysing capecitabine and streptozocin with or without cisplatin did also not show a statistically significant clinical benefit. ${ }^{4}$ Furthermore, in these trials, no improvement in toxicity, QoL or OS was seen, and therefore the ESMO-MCBS was not applicable.

Consensus in the ESMO-MCBS score was reached in two of six trials after the first scoring session. ${ }^{3-7,9,10}$ The variation in awarded scores was related to differences in interpretation of data, like the significance of the primary end point, and adjustment of the preliminary score for lack of improvement in QoL. After the second scoring session, consensus was reached in four of six trials. . $^{3-9,9-11}$

- Assessment according to the NHB-ASCO-F

Nine trials were assessable for the NHB-ASCO-F. ${ }^{3-12}$ The CBS varied from 0 to 63.8. In the trial reported by Raymond et al. and the RADIANT-3 and RADIANT-4 trials, the treatment arm experienced more adverse events, resulting in a toxicity score of -5 and -20 , respectively $[6,9 ., 10 ., 11$.$] . Bonus points were awarded for long-term$ disease control; if at a time point that was twice the median PFS for the control regimen, the percentage of patients having PFS was at least $50 \%$ higher for the intervention arm compared with the control arm, to the trial reported by Raymond et al., PROMID-trial, RADIANT-4, RADIANT-3 and the NETTER-1. ${ }^{6-11}$

No consensus was obtained for the score of the trials assessed by the NHB-ASCO-F after two scoring sessions. This was generally related to discrepancies in interpretation of toxicity score. Finally, after an additional discussion in four trials, consensus was obtained for NHB and in five trials consensus was reached for the CBS and bonus points. 


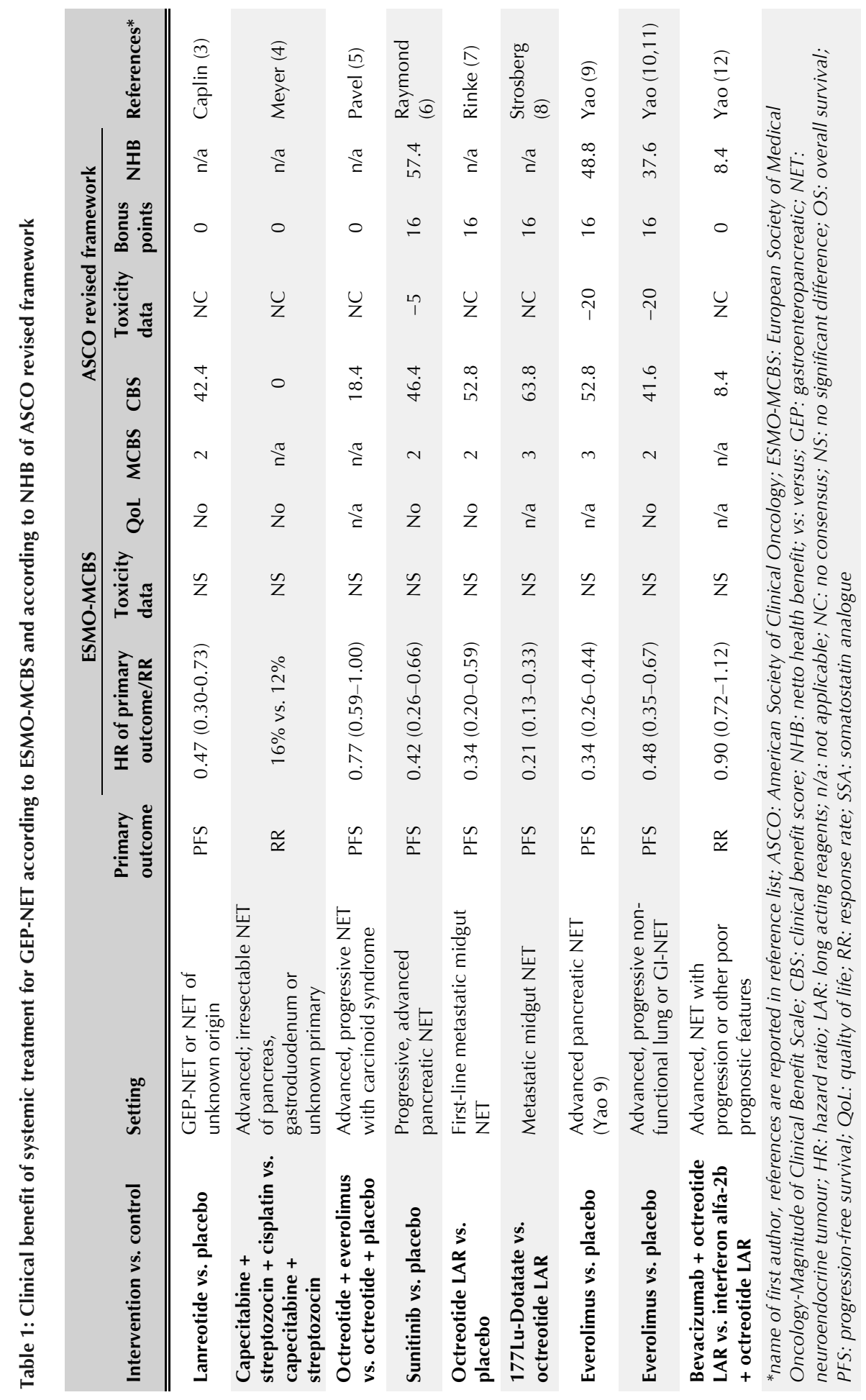




\section{Discussion}

To define the clinical benefit of systemic antitumor treatment in patients with GEPNETs, we systematically applied the ESMO-MCBS and the NHB-ASCO-F to relevant trials. Six out of 35 trials fulfilled all requirements to be assessed with the ESMOMCBS resulting in an ESMO-MCBS score of 2 or 3, while 9 trials could be assessed with the NHB-ASCO-F and resulted in scores between 37.6 and 57.4. No clear cutoff value to define clinical benefit with the ASCO framework was provided. ${ }^{15,16}$ None of the trials that were included in our analysis could demonstrate a meaningful clinical benefit according to the employed tools. The ESMO-MCBS scores were generally lower than in other tumour types, like metastasized breast or colorectal cancer. ${ }^{14,17}$ In more common tumour types, sufficient numbers of patients can be included in trials powered to detect a difference in OS between the intervention and control group. In NET, such large trials are scarce. In addition, OS difference detection is challenging in NET patients. Therefore, PFS is a frequently used primary end point in NET trials. An improvement in PFS has less impact compared with OS in the ESMO-MCBS scores and the NHB-ASCO-F. Furthermore, none of the assessable trials in GEP-NET showed an improvement in QoL or less toxicity as compared with the control arm. Therefore, these outcomes did not result in an upgrade of the preliminary score in the ESMO-MCBS.

Ideally, OS and QoL should be the primary end points of all trials with new drugs. ${ }^{20}$ This is challenging in NET. Reasons for this include the heterogeneous patient population, the long natural course of the disease and the wide variability of subsequent lines of therapy after progression. The heterogeneity of the patients composing the NET population is important to take into account. This illustrates the importance of well-defined inclusion criteria. The eligibility criteria of the RADIANT2 trial included patients with serotonin producing NET and progressive, low- or intermediate-grade and advanced disease. ${ }^{5}$ Despite these clear criteria, still large variation in patient and tumour characteristics between patients exists. Despite the randomized design of the trial, this could have influenced PFS.

Current guidelines for GEP-NET antitumour treatment are based on clinical trials and on expert opinion. These guidelines influence our daily clinical practice. Therefore, we also assessed trials that could not be fully assessed by the ESMO-MCBS and ASCO framework (supplementary Table S2). The control arms of trials analysing chemotherapy included interferon, dacarbazine and other types of chemotherapy, 
respectively. Given the current knowledge about the effects of chemotherapy, the control arm, nowadays, likely would not have contained chemotherapy. With this analysis, we demonstrated that current guidelines are partially based on studies not powered to determine end points that show clinical benefit.

Large trials were conducted by enormous efforts of the international NET community. ${ }^{3-12}$ This has provided us with important data. Improvements could include trials using a preselected patient population. This is already implemented in PRRT, where somatostatin receptor-positive patients are selected for treatment. ${ }^{8}$ Furthermore, sequential multiple assignment randomized trials can be used for hypothesis generation followed by a confirmatory RCT. ${ }^{21}$ In addition, trials should be focused on reporting QoL and be of sufficient follow-up duration. For patients with GEP-NETs, use of QoL could be of additional importance because OS is generally prolonged in this cohort.

The importance of long follow-up was previously demonstrated in a trial in patients with anaplastic oligodendrogliomas. OS of adjuvant chemoradiation therapy versus radiotherapy was not improved at a median follow-up of 60 months but was improved in the chemoradiation therapy arm at a median follow-up of 140 months. ${ }^{22}$

International collaboration in clinical trials and investigator-driven trials should be further expanded to increase evidence-based data to support treatment decisions and lead to meaningful clinical benefit for patients with GEP-NETs.

Although not the aim of our analysis, we found some limitations of the tools. The ESMO-MCBS downgrades the preliminary score of a trial if no significant improvement of QoL data was shown. ${ }^{14}$ If QoL was not reported, the preliminary score did not have to be downgraded. ${ }^{8-9}$

Furthermore, we noticed during the scoring process a difference in consensus achieved by assessing trials according to the ESMO-MCBS, compared with trials assessed according to the NHB-ASCO-F. With the ESMO-MCBS, for more trials consensus was achieved. A possible reason for this is the complex toxicity data reporting according to the NHB-ASCO-F. Potentially, a clearer definition of 'clinical relevant toxicity' could facilitate the scoring. With the ASCO framework, eight clinicians completed the tool for 11 anticancer drugs. A Cohen's kappa coefficient of the interrater reliability (ICC) of 0.11 for NHB and 0.06 for toxicity was found, corresponding with 'slightly reliable agreement' ${ }^{18}$ 
However, in another study where convergent validity and interrater reliability of assessment frameworks were analysed, the ASCO framework had an ICC of $0.80 .^{23}$ In another report, 109 RCTs were included and ESMO scores and scores using the concept and revised version of the ASCO framework were determined. Weak to moderate correlations were demonstrated, suggesting different constructs of clinical benefit measured. ${ }^{24}$ Other tools, used to define the value and also addressing the costs of drugs, include the National Comprehensive Cancer Network evidence blocks, Institute for Clinical and Economic Review value assessment framework and Memorial Sloan Kettering Drug Abacus. ${ }^{25-27}$ Every tool has its own aspects. In the future, converging of tools is expected which will allow to combine the best aspects of these tools to guide policy makers and patient-doctor discussions. ${ }^{28}$

\section{Conclusion}

The ESMO-MCBS and the NHB of ASCO revised framework could be applied, respectively, to only six and nine trials investigating systemic treatments in NET patients. The currently used systemic treatments for patients with GEP-NETs had low scores according to the NHB-ASCO-F, and none could be graded as meaningful clinical beneficial according to the ESMO-MCBS. Despite the low incidence of NET, the heterogeneous patient population and the relatively long natural course, future studies on new treatment modalities should aim for high clinical benefit outcomes. 


\section{References}

1. Mocellin S, Nitti D. Gastrointestinal carcinoid: epidemiological and survival evidence from a large population-based study $(n=25$ 531). Ann Oncol 2013;24(12):3040-3044.

2. Yao JC, Hassan M, Phan A et al. One hundred years after "carcinoid": epidemiology of and prognostic factors for neuroendocrine tumors in 35, 825 cases in the United States. J Clin Oncol 2008;26(18):3063-3072.

3. Caplin ME, Pavel M, Cwikła JB et al. Lanreotide in metastatic enteropancreatic neuroendocrine tumors. N Engl J Med 2014;371(3):224-233.

4. Meyer T, Qian W, Caplin ME et al. Capecitabine and streptozocin p/-cisplatin in advanced gastroenteropancreatic neuroendocrine tumours. Eur J Cancer 2014;50(5):902-911.

5. Pavel M, Oberg KE, Hainsworth JD et al. Everolimus plus octreotide long-acting release (LAR) for the treatment of advanced neuroendocrine tumors (NET) associated with carcinoid syndrome (RADIANT-2): updated overall survival results. Eur J Cancer 2013;49:S577.

6. Raymond E, Dahan L, Raoul JL et al. Sunitinib malate for the treatment of pancreatic neuroendocrine tumors. N Engl J Med 2011;364(6):501-513.

7. Rinke A, Wittenberg M, Schade-Brittinger $C$ et al. Placebo-controlled, double-blind, prospective, randomized study on the effect of octreotide LAR in the control of tumor growth in patients with metastatic neuroendocrine midgut tumors (PROMID): results of long-term survival. Neuroendocrinology 2017;104(1):26-32.

8. Strosberg J, El-Haddad G, Wolin E et al. Phase 3 trial of 177 Lu-dotatate for midgut neuroendocrine tumors. N Engl J Med 2017;376(2):125-135.

9. Yao JC, Pavel M, Lombard-Bohas C et al. Everolimus for the treatment of advanced pancreatic neuroendocrine tumors: overall survival and circulating biomarkers from the randomized, phase III RADIANT-3 study. J Clin Oncol 2016;34(32):3906-3913

10. Yao JC, Fazio N, Singh S et al. Everolimus for the treatment of advanced, nonfunctional neuroendocrine tumours of the lung or gastrointestinal tract (RADIANT-4): a randomised, placebo-controlled, phase 3 study. Lancet 2016;387(10022):968-977.

11. Pavel ME, Singh S, Strosberg JR et al. Health-related quality of life for everolimus versus placebo in patients with advanced, non-functional, well-differentiated gastrointestinal or lung neuroendocrine tumours (RADIANT-4): a multicentre, randomised, double-blind, placebo-controlled, phase 3 trial. Lancet Oncol 2017;18(10):1411-1422. 
12. Yao JC, Guthrie KA, Moran C et al. Phase III prospective randomized comparison trial of depot octreotide plus interferon alfa-2b versus depot octreotide plus bevacizumab in patients with advanced carcinoid tumors: SWOG S0518. J Clin Oncol 2017;35:1695-1703.

13. Kulke MH, Siu LL, Tepper JE et al. Future directions in the treatment of neuroendocrine tumors: consensus report of the National Cancer Institute Neuroendocrine Tumor clinical trials planning meeting. J Clin Oncol 2011;29:934943.

14. Cherny NI, Sullivan R, Dafni U et al. A standardised, generic, validated approach to stratify the magnitude of clinical benefit that can be anticipated from anti-cancer therapies: the European Society for Medical Oncology Magnitude of Clinical Benefit Scale (ESMO-MCBS). Ann Oncol 2015;26(8):1547-1573.

15. Schnipper LE, Davidson NE, Wollins DS et al. American Society of Clinical Oncology Statement: a conceptual framework to assess the value of cancer treatment options. J Clin Oncol 2015;33:2563-2577.

16. Schnipper LE, Davidson NE, Wollins DS et al. Updating the American Society of Clinical Oncology Value Framework: revisions and reflections in response to comments received. J Clin Oncol 2016;34:2925-2934.

17. Kiesewetter B, Raderer M, Steger GG et al. The European Society for Medical Oncology Magnitude of Clinical Benefit Scale in daily practice: a single institution, real-life experience at the Medical University of Vienna. ESMO Open 2016;1(4):e000066.

18. Wilson L, Lin T, Wang L et al. Evaluation of the ASCO value framework for anticancer drugs at an academic medical center. J Manag Care Spec Pharm 2017;23(2):163-169.

19. Ramage J, Punia P, Faluyi O et al. UK phase IV, observational study to assess quality of life in patients (pts) with pancreatic neuroendocrine tumours (pNETS) receiving treatment with everolimus: The "real-world" OBLIQUE study. Ann Oncol 2016;27(Suppl 6):437.

20. Booth CM, Eisenhauer EA. Progression-free survival: meaningful or simply measurable? J Clin Oncol. 2012;30(10):1030-1033.

21. Moodie EE, Karran JC, Shortreed SM. A case study of SMART attributes: a qualitative assessment of generalizability, retention rate, and trial quality. Trials 2016;17(1):242.

22. van den Bent MJ, Brandes AA, Taphoorn MJ et al. Adjuvant procarbazine, lomustine, and vincristine chemotherapy in newly diagnosed anaplastic oligodendroglioma: long-term follow-up of EORTC brain tumor group study 26951. J Clin Oncol 2013;31:344-350. 
Part II | Chapter 6

23. Bentley TGK, Cohen JT, Elkin EB et al. Measuring the value of new drugs: validity and reliability of 4 value assessment frameworks in the oncology setting. J Manag Care Spec Pharm 2017;23:S34-S48.

24. Cheng S, McDonald EJ, Cheung MC et al. Do the American Society of Clinical Oncology Value Framework and the European Society of Medical Oncology Magnitude of Clinical Benefit Scale measure the same construct of clinical benefit? J Clin Oncol 2017;25. NCCN Evidence Blocks. National Comprehensive Cancer Network. https://www.nccn.org/evidenceblocks/ (28 September 2017, date last accessed).

26. Drug Abacus. Memorial Sloan Kettering Cancer Center, 2016. https:// drugpricinglab.org/tools/drug-abacus/ (28 September 2017, date last accessed).

27. The ICER approach. Institute for Clinicial and Economic Review, 2017. https://icer-review.org/methodology/icers-methods/.

28. Schnipper LE, Schilsky RL. Converging on the value of value frameworks. J Clin Oncol 2017;35(24):2732-2734. 


\section{Supplementary Appendix S1: Extensive methods \& search strategy}

Trials were searched in the databases PubMed and EMBASE between Aug 1st 2015 to Jan 31 th 2016. The following search terms were used in various combinations 'neuroendocrine', 'tumor', 'pNET', 'neoplasm', 'survival', 'disease-free survival', 'quality of life', 'QOL', 'toxicity', 'adverse events', 'chemotherapy', 'cytostatic', 'interferon', 'somatostatin analogue', 'everolimus', 'sunitinib', 'indium', 'lutetium', 'peptide receptor radionuclide', 'controlled trial' and 'clinical trial'. The articles that were found were screened using title and abstract to select trials published in the English language and comparing systemic treatment modalities for the treatment of GEP-NET in humans. Furthermore the reference lists of the European Neuroendocrine Tumour Society (ENETS), National Comprehensive Cancer Network, (NCCN) and North American Neuroendocrine Tumour Society (NANETS), guidelines were reviewed for comparative trials that support the guidelines (suppl ref 27-29). Also reference lists of included trials and conference abstracts were reviewed for additional relevant articles.

\section{Selection criteria for trials}

Comparative trials investigating systemic antitumor treatment for patients with GEPNETs were analysed. Criteria for assessment are summarized in Table S1. Trials were eligible for grading with the ESMO-MCBS if either a randomized or comparative cohort design was used or if a meta-analysis was used reporting a statistically significant benefit in any of the evaluated outcomes. With the NHB of the revised ASCO framework only randomized controlled trials (RCTs) could be graded. Furthermore for both tools only well-powered trials evaluating survival, QoL, surrogate outcomes of survival (disease- free interval, event-free survival, time to response, PFS and time to progression), or treatment toxicity could be graded. When more than one trial, that fulfilled the selection criteria, analysed the same clinical question, results derived from well powered registration trials, prevailed. Trials were selected if at least $50 \%$ of participants were diagnosed with GEP-NET, and the other participants had a NET of unknown or other origin. Abstracts reporting clinical trials in patients with GEP-NETs were only permitted for analysis if they showed additional data to an already published article.

ESMO-MCBS and the NHB of the revised ASCO framework

ESMO-MCBS grades in the non-curative setting ranges from 1-5, with grade 4 and 5 representing meaningful clinical benefit. To evaluate different endpoints three forms 
are available; form $2 \mathrm{a}$ and $2 \mathrm{~b}$ for 'trials with primary endpoint $\mathrm{OS}^{\text {', }}$ and 'PFS', respectively and form 2c for trials with 'with primary endpoint other than OS or PFS or equivalence studies'. The NHB of the revised ASCO framework consists of the clinical benefit score (CBS), (i.e. the hazard ratio or median of OS or PFS, or response rate $(R R)$ ) toxicity and bonus points (for long term disease control, palliation, treatment free interval and QoL). The NHB of the revised ASCO framework ranges from -20 to 180, with a higher score representing a better NHB, no cut off value was provided to define clinical benefit. All relevant comparative trials were assessed according to both tools. Relevant trials that did not meet all criteria as mentioned in Table 1 were assessed separately, based on the available data.

\section{Assessment of trials}

Four members from all Dutch European Neuroendocrine Tumour Society (ENETS) centers of excellence (Academic Medical Center, Erasmus Medical Center, the Netherlands Cancer Institute, and University Medical Center Groningen) independently scored the trials according to the ESMO-MCBS and the NHB of the revised ASCO framework. After obtaining scores of these assessors we noticed a wide variation in results. During a consensus meeting additional agreements were defined. Next, trials were assessed again according to the additional agreements. The number of trials to which the same score was awarded between three or four assessors was registered after each of the two scoring sessions. Two phase III trials were published after the consensus meeting and were assessed according our agreements. Therefore an additional assessment session was not necessary for these trials. 


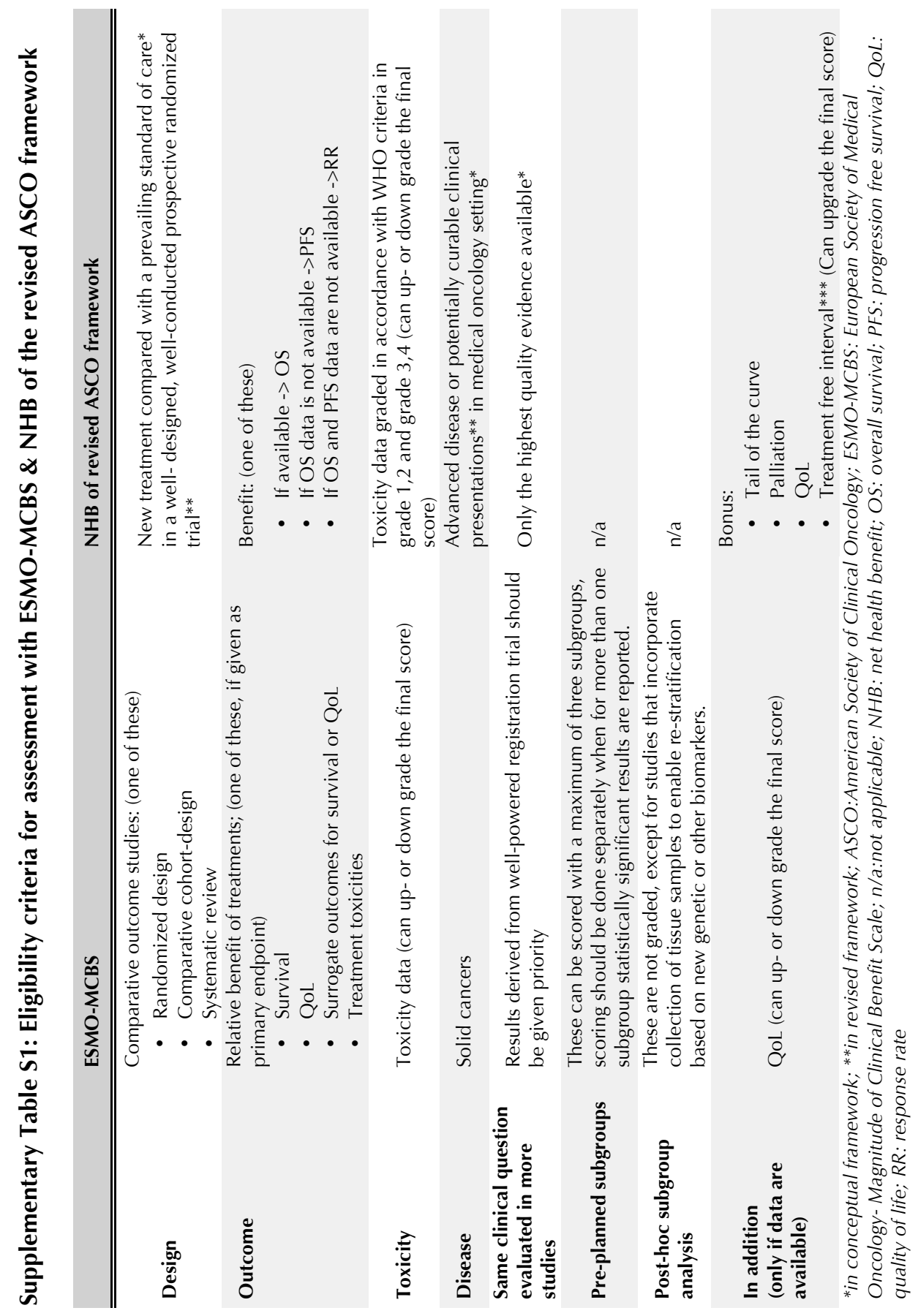




\section{Supplementary Figure S1: Inclusion process for the literature analysis}

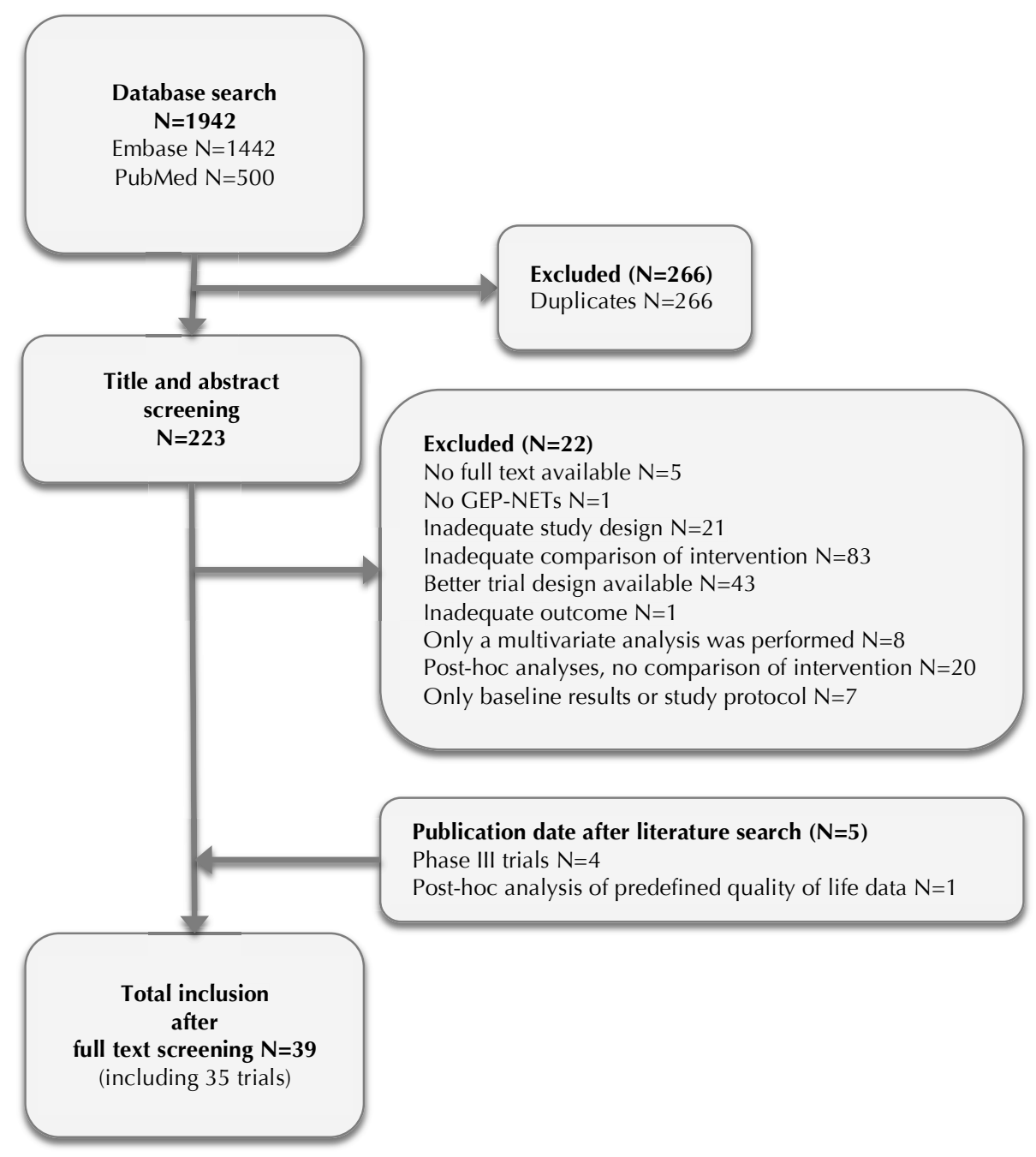

GEP-NET: gastrointestinal or pancreatic neuroendocrine tumour 
Supplementary Table S2: Eligibility criteria for a valid evaluation with the tools

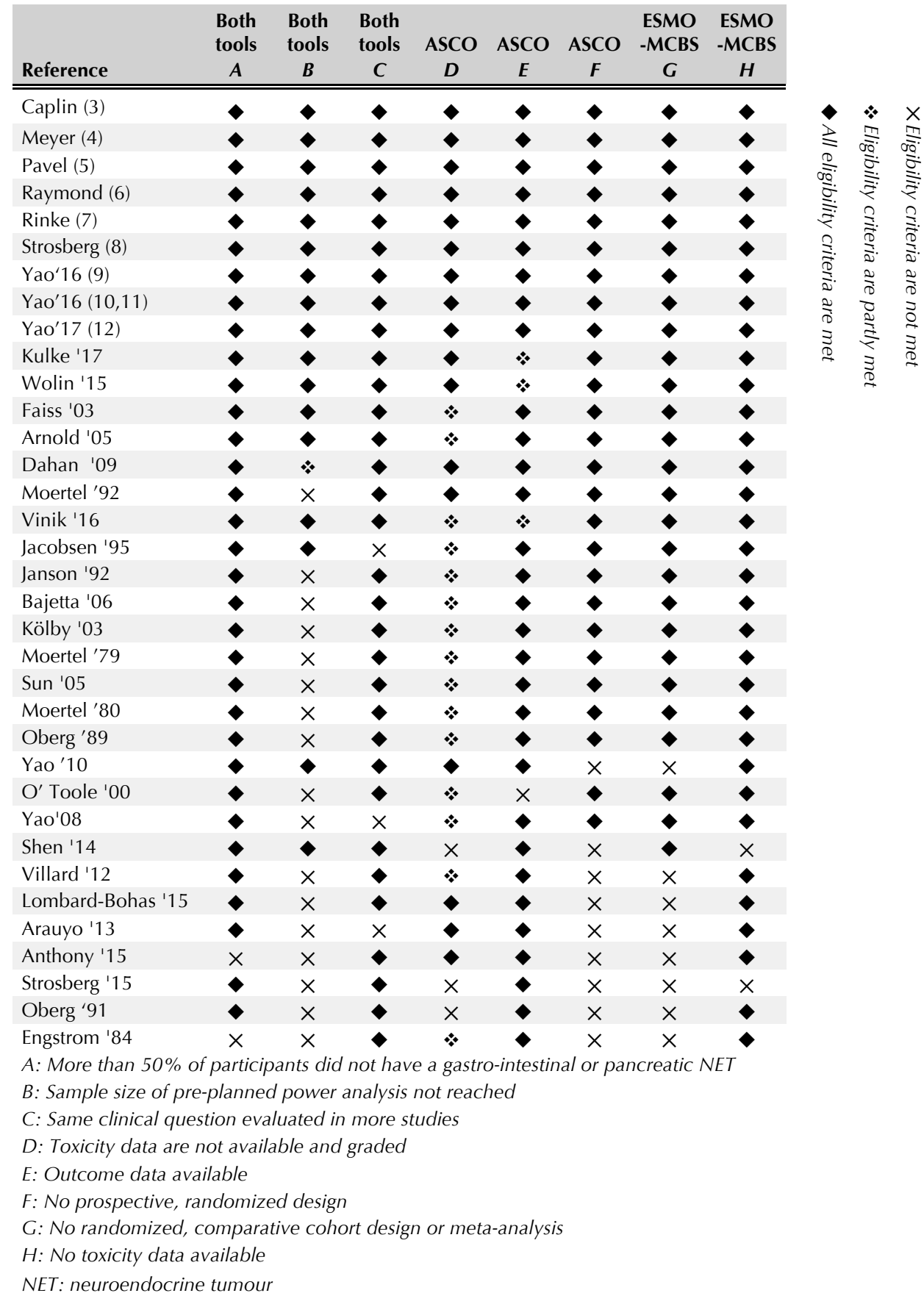




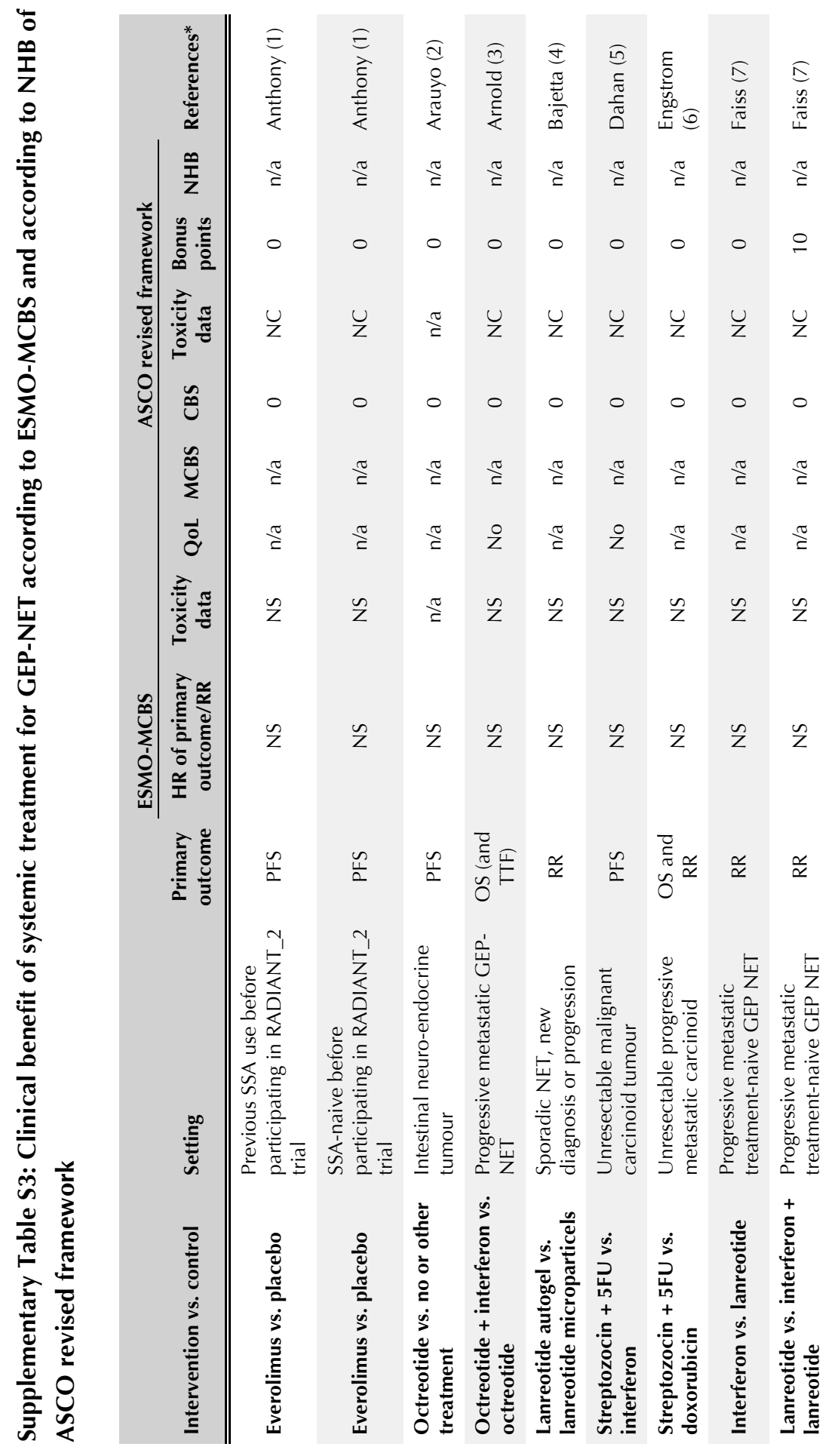




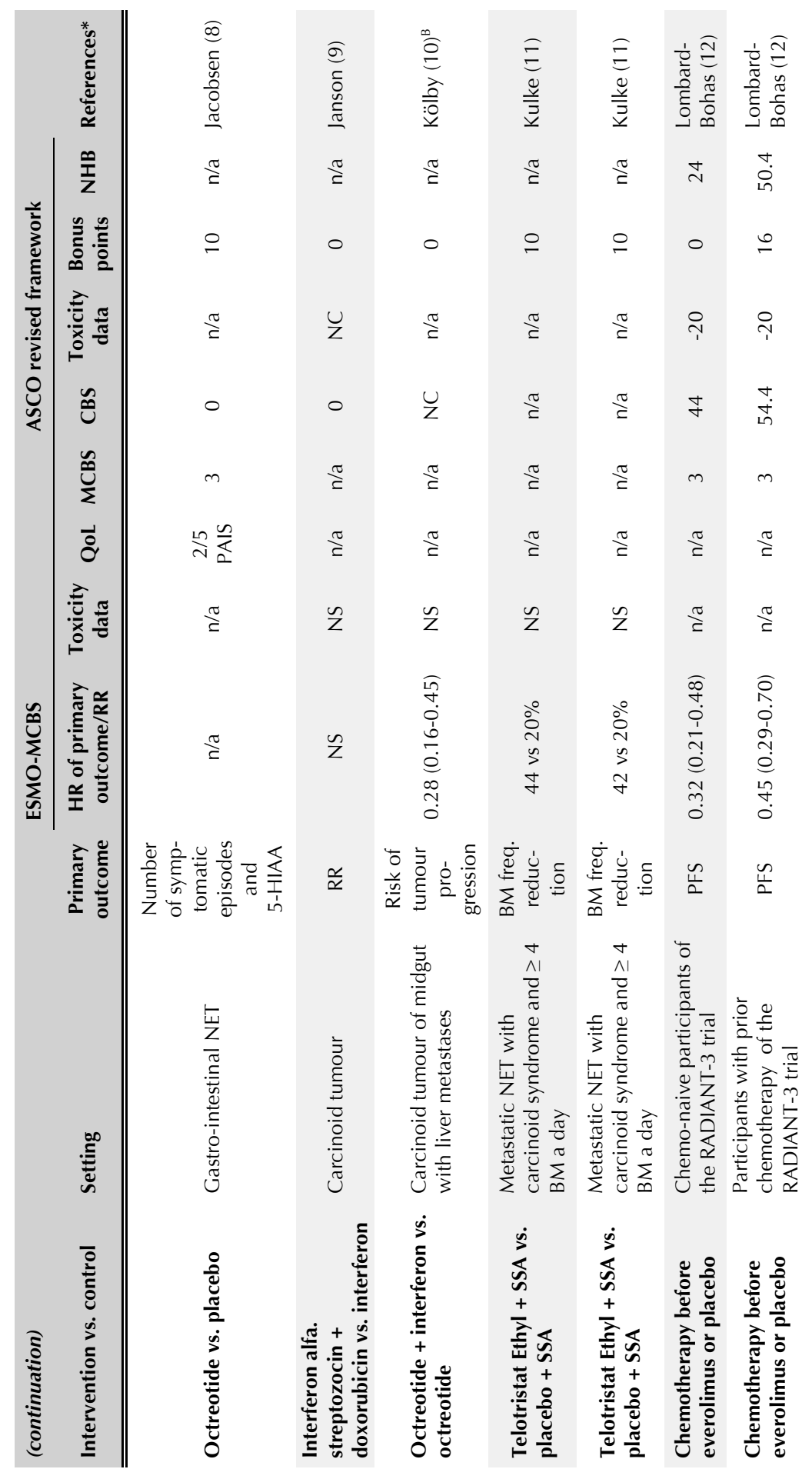




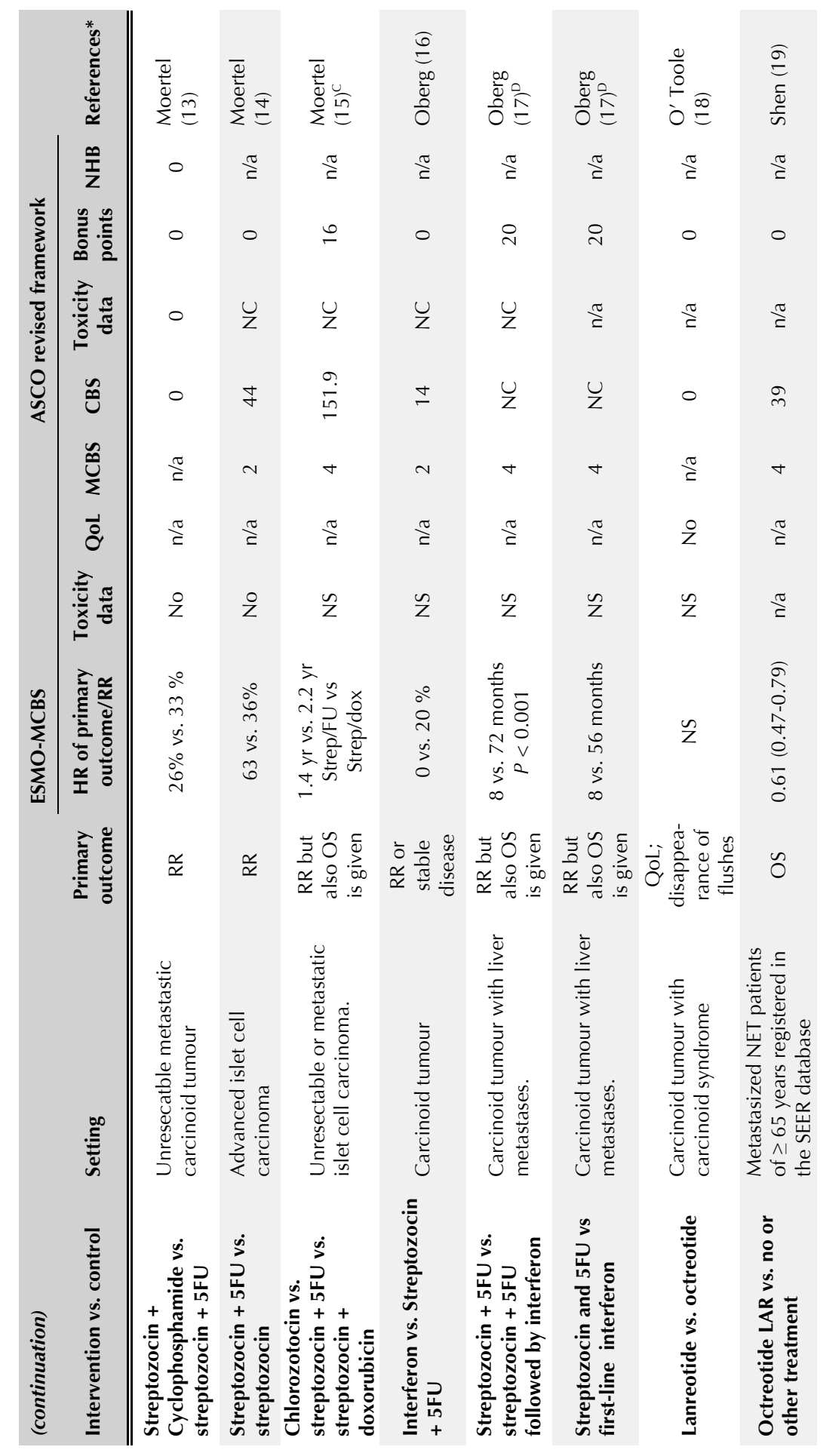




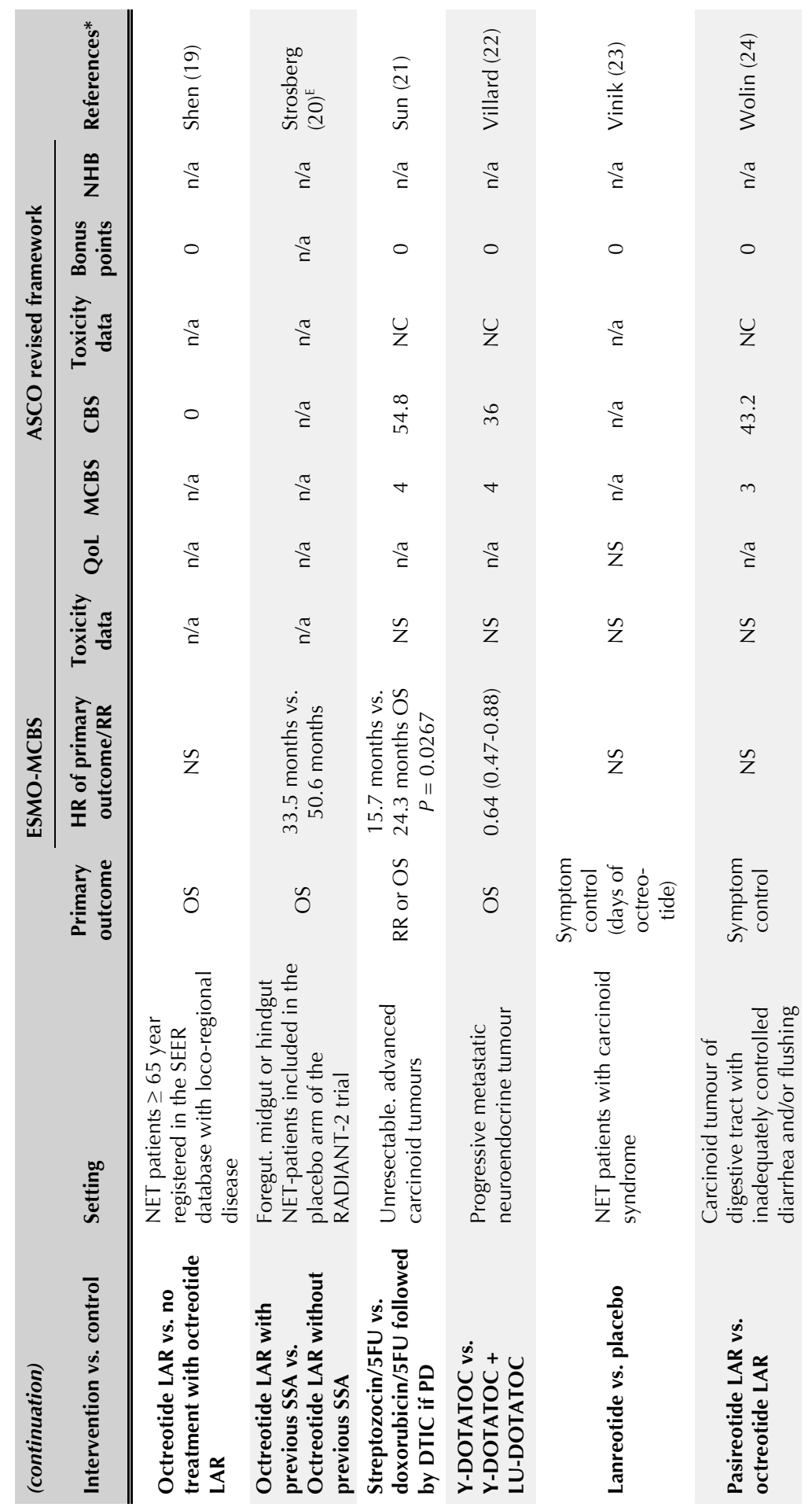




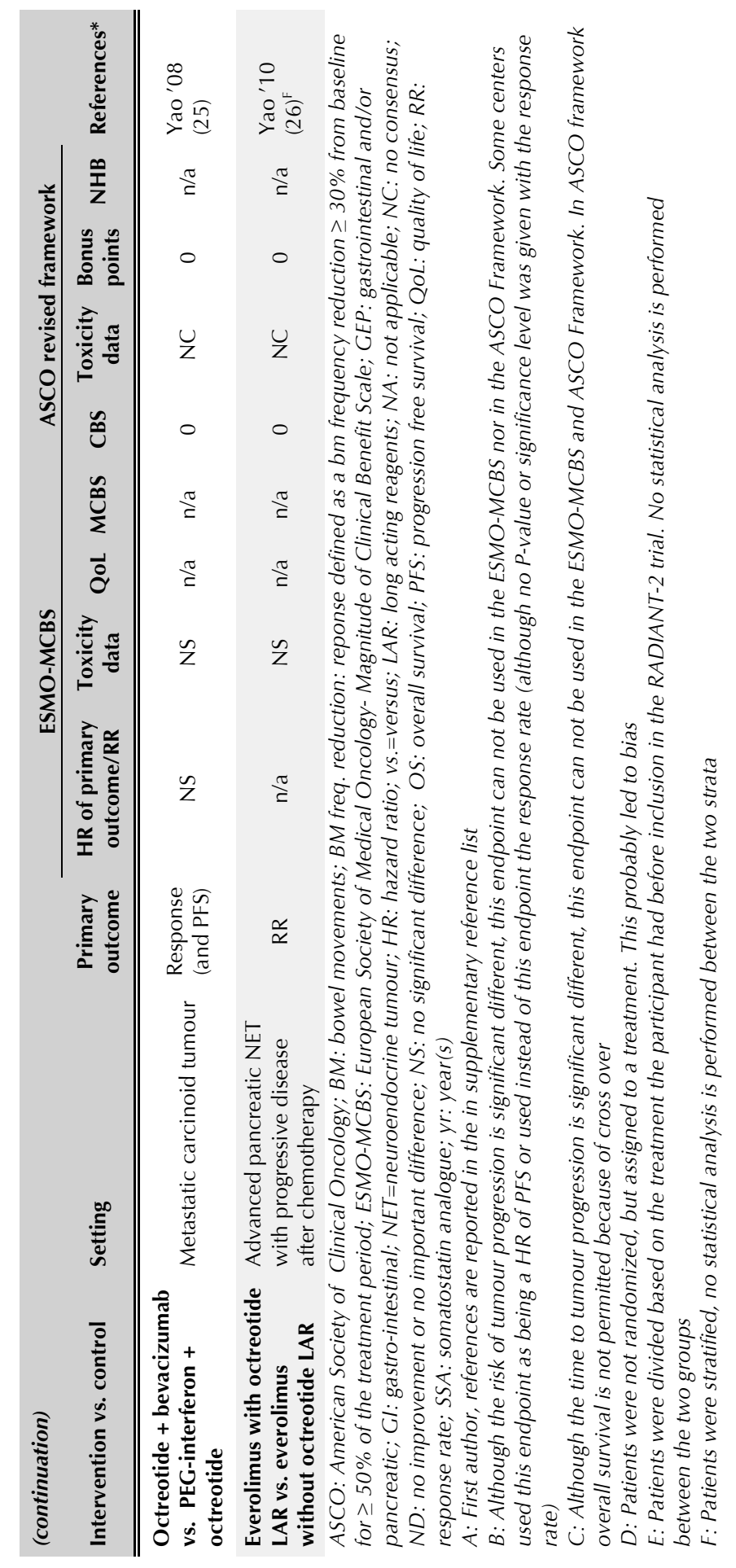




\section{Supplementary Appendix S2: References}

1. Anthony LB, Pavel ME, Hainsworth JD, et al. Impact of previous somatostatin analogue use on the activity of everolimus in patients with advanced neuroendocrine tumors: Analysis from the phase III RADIANT-2 trial. Neuroendocrinology 2015;102:18-25.

2. Araujo PB, Cheng S, Mete O, et al. Evaluation of the WHO 2010 grading and AJCC/UICC staging systems in prognostic behaviour of intestinal neuroendocrine tumors. PLoS One 2013;8:e61538.

3. Arnold R, Rinke A, Klose KJ, et al. Octreotide versus octreotide plus interferon-alpha in endocrine gastroenteropancreatic tumors: a randomized trial. Clin Gastroenterol Hepatol 2005;3:761-71.

4. Bajetta E, Procopio G, Catena L, et al. Lanreotide autogel every 6 weeks compared with Lanreotide microparticles every 3 weeks in patients with well differentiated neuroendocrine tumors: a Phase III Study. Cancer 2006;107:2474-81.

5. Dahan L, Bonnetain F, Rougier P, et al. Phase III trial of chemotherapy using 5fluorouracil and streptozotocin compared with interferon alpha for advanced carcinoid tumors: FNCLCC-FFCD 9710. Endocr Relat Cancer 2009;16:1351-61.

6. Engstrom PF, Lavin PT, Moertel CG, Folsch E, Douglass HO,Jr. Streptozocin plus fluorouracil versus doxorubicin therapy for metastatic carcinoid tumor. J Clin Oncol 1984;2:1255-9.

7. Faiss S, Pape UF, Bohmig M, et al. Prospective, randomized, multicenter trial on the antiproliferative effect of lanreotide, interferon alfa, and their combination for therapy of metastatic neuroendocrine gastroenteropancreatic tumors--the International Lanreotide and Interferon Alfa Study Group. J Clin Oncol 2003;21:2689-96.

8. Jacobsen MB, Hanssen LE. Clinical effects of octreotide compared to placebo in patients with gastrointestinal neuroendocrine tumours. Report on a double-blind, randomized trial. J Intern Med 1995;237:269-75.

9. Janson ET, Ronnblom L, Ahlstrom H, et al. Treatment with alpha-interferon versus alpha- interferon in combination with streptozocin and doxorubicin in patients with malignant carcinoid tumors: a randomized trial. Ann Oncol 1992;3:635-8.

10. Kolby L, Persson G, Franzen S, Ahren B. Randomized clinical trial of the effect of inter- feron alpha on survival in patients with disseminated midgut carcinoid tumours. Br J Surg 2003;90:687-93.

11. Kulke MH, Horsch D, Caplin ME, et al. Telotristat ethyl, a tryptophan hydroxylase inhibitor for the treatment of carcinoid syndrome. J Clin Oncol 2017;35:14-23. 
12. Lombard-Bohas C, Yao JC, Hobday T, et al. Impact of prior chemotherapy use on the efficacy of everolimus in patients with advanced pancreatic neuroendocrine tumors: a subgroup analysis of the phase III RADIANT-3 trial. Pancreas 2015;44:181-9.

13. Moertel CG, Hanley JA. Combination chemotherapy trials in metastatic carcinoid tumor and the malignant carcinoid syndrome. Cancer Clin Trials 1979;2:327-34.

14. Moertel CG, Hanley JA, Johnson LA. Streptozocin alone compared with streptozocin plus fluorouracil in the treatment of advanced islet-cell carcinoma. N Engl J Med 1980;303:1189- 94.

15. Moertel CG, Lefkopoulo M, Lipsitz S, Hahn RG, Klaassen D. Streptozocindoxorubicin, streptozocin- fluorouracil or chlorozotocin in the treatment of advanced islet-cell carcinoma. N Engl J Med 1992;326:519-23.

16. Oberg K, Norheim I, Alm G. Treatment of malignant carcinoid tumors: a randomized con- trolled study of streptozocin plus 5-FU and human leukocyte interferon. Eur J Cancer Clin Oncol 1989;25:1475-9.

17. Oberg K, Eriksson B. The role of interferons in the management of carcinoid tumours. Br J Haematol 1991;79 Suppl 1:74-7.

18. O'Toole D, Ducreux M, Bommelaer G, et al. Treatment of carcinoid syndrome: a prospective crossover evaluation of lanreotide versus octreotide in terms of efficacy, patient accept-ability, and tolerance. Cancer 2000;88:770-6.

19. Shen C, Shih YC, Xu Y, Yao JC. Octreotide long-acting repeatable use among elderly pa-tients with carcinoid syndrome and survival outcomes: a population-based analysis. Cancer 2014;120:2039-49.

20. Strosberg JR, Yao JC, Bajetta E, et al. Efficacy of octreotide long-acting repeatable in neuroendocrine tumors: RADIANT-2 placebo arm post hoc analysis. Endocr Relat Cancer 2015;22:933-40.

21. Sun W, Lipsitz S, Catalano P, Mailliard JA, Haller DG, Eastern Cooperative Oncology Group. Phase II/III study of doxorubicin with fluorouracil compared with streptozocin with fluorouracil or dacarbazine in the treatment of advanced carcinoid tumors: Eastern Coop- erative Oncology Group Study E1281. J Clin Oncol 2005;23:4897-904.

22. Villard L, Romer A, Marincek N, et al. Cohort study of somatostatin-based radiopeptide therapy with [90Y-DOTA]-TOC versus [90Y-DOTA]-TOC plus [177LuDOTA]-TOC in neuroen-docrine cancers. J Clin Oncol 2012;30:1100-6.

23. Vinik AI, Wolin EM, Liyanage N, Gomez-Panzani E, Fisher GA, ELECT Study Group *. Eval-uation of lanreotide depot/autogel efficacy and safety as a carcinoid syndrome treatment (elect): a randomized, double-blind, placebo-controlled trial. Endocr Pract 2016;22:1068- 80. 
24. Wolin EM, Jarzab B, Eriksson B, et al. Phase III study of pasireotide long-acting release in patients with metastatic neuroendocrine tumors and carcinoid symptoms refractory to available somatostatin analogues. Drug Des Devel Ther 2015;9:5075-86.

25. Yao JC, Phan A, Ho PM, et al. Targeting vascular endothelial growth factor in advanced carcinoid tumor: a random assignment phase II study of depot octreotide with bevacizumab and pegylated interferon alpha-2b. J Clin Oncol 2008;26:1316-23.

26. Yao JC, Lombard-Bohas C, Baudin E, et al. Daily oral everolimus activity in patients with metastatic pancreatic neuroendocrine tumors after failure of cytotoxic chemotherapy: a phase II trial. J Clin Oncol 2010;28:69-76.

27 ENETS Guidelines 2016. (at http://www.enets.org/current_guidelines.html).

28. NCCN Guidelines 2017. (at https://www.nccn.org/professionals/physician_gls/pdf/neuroen-docrine.pdf).

29. Kunz PL, Reidy-Lagunes D, Anthony LB, et al. Consensus guidelines for the management and treatment of neuroendocrine tumors. Pancreas 2013;42:557-77. 


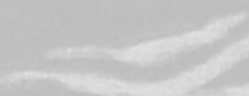

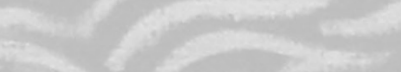

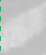

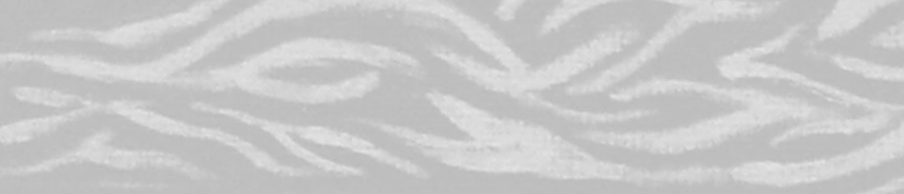




\section{Chapter 7}

\section{Comparison of transarterial bland embolization and}

selective internal radiation therapy in patients with metastatic neuroendocrine tumours

L.M. van Veenendaal, K.G. Samsom, A.J.A.T. Braat, J. Verdult, W. Prevoo, W.H.

Verbeek, G.D.Valk, M.R. Vriens, M.G.E.H. Lam, M.E.T. Tesselaar

Submitted for publication 


\section{Abstract}

\section{Background}

Transarterial embolization (TAE) and selective internal radiation therapy (SIRT) are treatment modalities for patients with liver metastases of a neuroendocrine tumour (NET). The safety and effectiveness of TAE and SIRT have scarcely been compared.

\section{Patients and Methods}

Patients with well-differentiated NET undergoing TAE or SIRT during 2004-2016 were analysed. Toxicity and response were evaluated during 3 months of follow-up according to CTCAE v4.03 and RECIST 1.1. Survival was analysed by Kaplan Meier curves and log-rank test. Subgroup analyses were performed for small intestinal (SINET) and pancreatic NET (pNET).

\section{Results}

86 Patients (39 males, median age 60 years) with 130 procedures (90 TAE; 40 SIRT) were included. The treatment cohorts had different characteristics (SI-NET: 65\% vs. $34 \%$, pNET: 15 vs. $34 \%$, functional symptoms: $74 \%$ vs. $41 \%$, for TAE and SIRT respectively). Grade 1-2 toxicity occurred in $2-72 \%$ vs. $5-60 \%$ and grade $3-4$ toxicity occurred in $1-47 \%$ vs. $3-13 \%$ for TAE and SIRT, respectively. Functional symptoms improved after TAE in the whole cohort $(P=0.002)$ and in SI-NETS $(P=0.02)$. No difference in survival was observed.

\section{Conclusion}

TAE and SIRT are safe and effective. After TAE, severe adverse events occurred frequently. However, TAE might reduce functional symptoms. No differences in survival rates were observed. 


\section{Introduction}

Neuroendocrine tumours (NET) represent a heterogeneous group of rare tumours. They most commonly develop in the gastrointestinal tract and bronchopulmonary system. ${ }^{1-3}$ The incidence of NETs is increasing, with a reported incidence of 5.25 per 100.000 individuals in 2004. ${ }^{1}$ Most NETs show an indolent but heterogeneous disease course. Nowadays, the only potentially curative treatment for NETs consists of surgery. Approximately $38 \%$ of patients have metastases at time of diagnosis, predominantly of the liver. ${ }^{1,2}$ Only a minority of patients (20-30\%) with metastasized NETs (mNETs) is eligible for curative surgery. ${ }^{4,5}$ Furthermore, in patients who underwent liver surgery with curative intent, diffuse microscopic involvement is often seen ( $>50 \%$ of patients) and during the course of their disease, and an estimated 25 $93 \%$ of these patients will ultimately develop liver metastases (LM). ${ }^{3,6-8}$

The presence of $L M$ is a strong negative prognostic factor and causes significant morbidity for NET patients. ${ }^{5,6,9}$ The 5-year overall survival (OS) for NET patients with untreated LM is reported to be $24-52 \%$ compared to approximately $75 \%$ for patients with localized resectable disease. ${ }^{2,3,7}$ In case of $L M$, serotonin and other vasoactive mediators can not be (completely) metabolized by the liver, inducing functional symptoms e.g. carcinoid syndrome. The carcinoid syndrome is characterised by diarrhoea, episodic flushing, bronchospasm and right valvular heart failure. In case of insulinomas, hypoglycaemia can occur. Additionally, LM can gradually replace the liver parenchyma, which is detrimental to its function and will lead to liver failure and subsequent death. Therefore, treatment strategies to obtain control of LM are not only relevant in terms of prognosis but also to achieve symptom control, which could increase quality of life.

Transarterial liver directed therapies (LDT) are widely adopted in NET patients with liver dominant metastases..$^{5,10-12}$ LDT exploits the nearly exclusive blood supply of neuroendocrine LM from the hepatic artery, whereas normal liver parenchyma mainly derives its blood supply from the portal vein. ${ }^{4,13}$ Inducing ischemia with particles in case of trans arterial embolization (TAE) or particles with a radioactive isotope for irradiation in case of selective internal radiation therapy (SIRT), are both administered intra-arterially thereby selectively targeting the LM, limiting systemic and hepatic toxicity. 
Currently, TAE and SIRT are considered safe and effective treatment modalities as they provide objective tumour responses, symptom relief and decrease in tumour markers. ${ }^{4,13,14}$ However, differences between TAE and SIRT in NET patients have scarcely been investigated. Therefore, the aim of this study is to evaluate the safety and efficacy of TAE and SIRT in NET patients with liver-dominant disease.

\section{Patients and methods}

This study was conducted in accordance with institutional medical ethical guidelines. Between 2004 and 2016, all consecutive patients with histologically proven well differentiated NET with liver-dominant disease undergoing TAE or SIRT in the Netherlands Cancer Institute $(\mathrm{NCl})$ - University Medical Center Utrecht (UMCU), an European Neuroendocrine Tumour Society Center of Excellence (ENETS $\mathrm{CoE})$, were evaluated retrospectively. Patient medical records were screened and data on clinical and procedural characteristics were collected. Tumour grade was classified according to the World Health Organization (WHO) 2010 classification since this classification was in place at the time of diagnosis. ${ }^{13}$ The presence of functional symptoms and the use of somatostatin analogues (SSA) were retrieved from electronic patient charts. Time to LDT was calculated from date of diagnosis to date of either TAE or SIRT.

Patients received TAE or SIRT depending on primary treatment center. In the UMCU, NET patients were treated with SIRT, whereas patients in the $\mathrm{NCI}$ underwent TAE. Experienced interventional radiologists (all with more than 5 years of experience) performed all procedures. Both embolization techniques encompass the injection of either embolic or radioactive particles by means of a micro catheter positioned in the arterial vasculature of the liver under fluoroscopy. Depending on tumour burden and location of metastases, embolization was either performed in a single session or in a sequential lobar fashion. Embolization procedures (predominantly whole liver treatment in 2 tempi) performed within 3 months of each other were considered one (sequential) procedure.

- Trans arterial embolization

Pre-procedural, contrast enhanced multiphase CT or MRI imaging and angiography took place to evaluate the liver parenchyma (volume), location of metastases and 
vasculature (patency of the portal vein). TAE was performed using polyvinyl alcohol (Ivalon $\AA$, Bead Block $\AA$, Drivalon $\AA$ ) or polyzene-F hydrogel (Embozene $\AA$, Celanova $\left.{ }^{\circledR}\right)$ particles. TAE was performed using particles of sizes between 100 and $1000 \mu \mathrm{m}$ until the selected vessel demonstrated complete or near complete stasis of flow.

- Selective internal radiation therapy

Hepatic angiography and 99mTc-macroaggregated albumin (MAA) simulation and MAA scanning were performed prior to SIRT to exclude extrahepatic activity and lung shunting. SIRT was performed using resin (SIR-Spheres ${ }^{\circledR}$, Sirtex Medical) or glass (Theraspheres ${ }^{\circledR}$, BTG International) $90 \mathrm{Y}$ microspheres. Activity calculations in SIRT were based on the body surface area method or MIRD, according to the appropriate instructions for use. Prophylactic intravenous Octreotide infusion or prophylactic antibiotic treatment was given at the discretion of the treating physician and according to institutional guidelines.

\section{- Toxicity, Response and Overall Survival}

The duration of follow-up for toxicity and response was at least 3 months after TAE and SIRT. After 1 month and 3 months clinical evaluation, laboratory tests and CT (triple phase or four phase) of the liver were obtained. In case of sequential procedures follow-up for toxicity started after the first procedure and ended 3 months after the final sequential procedure. The response evaluation took place 3 months after the final procedure.

Post-embolization toxicity included all clinical and biochemical adverse events graded according to the Common Terminology Criteria for Adverse Events (CTCAE v4.03). ${ }^{15}$ Grade 3 toxicities or higher were considered severe. The biochemical follow-up included bilirubin, aspartate aminotransferase (ASAT) and alanine aminotransferase (ALAT). For each patient, baseline and the highest CTCAE toxicity grade during 3 months of follow-up was determined.

For radioembolization induced liver disease (REILD), we used the definition recommended by Braat et al: 'a symptomatic post-RE deterioration in the ability of the liver to maintain its (normal or preprocedural) synthetic, excretory, and detoxifying functions. It is characterised by jaundice and the development of or increase in ascites, hyperbilirubinemia, and hypoalbuminemia developing at least 2 
weeks - 4 months after RE, in the absence of tumour progression or biliary obstruction. ${ }^{16}$

Radiological response was defined using the Response Evaluation Criteria in Solid Tumours (RECIST, version 1.1). ${ }^{17}$ An independent radiologist, blinded for intervention and patient characteristics, conducted an anonymized assessment of the baseline and follow-up radiological images. Also baseline tumour volume of the LM was determined. Biochemical response was defined as $>50 \%$ decrease in Chromogranin A. A clinical response was defined as a decrease in use of somatostatin analogues (dosage or frequency) or decrease in reported functional symptoms. The total response rate was defined as at least one type of response, either clinical, biochemical or radiological response. A sub-group analysis was performed for small intestinal NET (SI-NET) and pancreatic NET (pNET). Overall survival (OS) was calculated from date of the (first) procedure until death.

- Statistical analysis

Descriptive analyses were performed to summarize patient demographics and treatment characteristics. For response evaluation a Chi-square analysis was used. A Fisher exact test was performed in case of groups $<10$ patients. Survival analyses were performed using Kaplan Meier curves and log rank test. Patients who were lost to FU before reaching one of the endpoints were censored at their last time of FU. A commercial statistical software package (SPSS for Windows, version 23.0; SPSS Inc, Chicago, Illinois) was used for data analysis. A P value $<0.05$ was considered statistically significant.

\section{Results}

A total of 86 patients underwent LDT, of whom 54 patients (63\%) underwent TAE and 32 patients $(37 \%)$ underwent SIRT. Patients had a median age of 60 years and females were slightly overrepresented (55\%). The median FU period was 19 months (IQR 7-26). A total of $81 \%$ of patients had a gastroenteropancreatic NET (GEP-NET). At time of diagnosis $79 \%$ of the patients had LM and LDT was performed 58 months (median) after diagnosis. The occurrence of SI-NETs and pNETs differed between the TAE and SIRT cohort (65\% vs $34 \%$ and $15 \%$ vs $34 \%$, respectively). Subsequently, a higher rate of functional symptoms was seen in the TAE cohort $(74 \%)$ compared to 
the SIRT cohort (41\%). Previous therapies consisted mostly of surgery (90\%) and the use of SSAs (83\%). A more detailed overview of the baseline characteristics can be found in Table 1.

In total, 130 procedures were performed. Procedural characteristics can be found in Table 2. Most TAE procedures targeted the hemi-liver or multiple selected liver segments $(89 \%)$, while almost half of the patients $(41 \%)$ underwent whole liver SIRT. In both treatment groups, $91 \%$ of patients underwent a single treatment. A sequential treatment strategy was performed in $7 \%$ and $9 \%$ in TAE and SIRT group, respectively. In seven patients who underwent SIRT, resin particles were used in 9 procedures.

The median administered activity was $3085 \mathrm{MBq}$ when glass particles were used and $1183 \mathrm{MBq}$ when resin particles were used.

Table 1: Baseline characteristics

\begin{tabular}{|c|c|c|c|c|c|c|}
\hline Characteristic & All & $\%$ or IQR & TAE & $\%$ or IQR & SIRT & $\%$ or IQR \\
\hline $\mathbf{N}$ & 86 & $100 \%$ & 54 & $63 \%$ & 32 & $37 \%$ \\
\hline Male & 39 & $45 \%$ & 24 & $44 \%$ & 15 & $47 \%$ \\
\hline Female & 47 & $55 \%$ & 30 & $56 \%$ & 17 & $53 \%$ \\
\hline Age (years) & 60 & $52-67$ & 60 & $53-67$ & 61 & $50-67$ \\
\hline \multicolumn{7}{|l|}{ Origin: } \\
\hline GEP-NET & 81 & $94 \%$ & 51 & $94 \%$ & 30 & $94 \%$ \\
\hline Small intestine & 46 & $54 \%$ & 35 & $65 \%$ & 11 & $34 \%$ \\
\hline UP & 8 & $9 \%$ & 3 & $6 \%$ & 5 & $16 \%$ \\
\hline pNET & 19 & $22 \%$ & 8 & $15 \%$ & 11 & $34 \%$ \\
\hline Other & 8 & $9 \%$ & 5 & $6 \%$ & 3 & $9 \%$ \\
\hline Lung & 5 & $6 \%$ & 3 & $6 \%$ & 2 & $6 \%$ \\
\hline Liver metastases & 66 & $79 \%$ & 42 & $78 \%$ & 24 & $75 \%$ \\
\hline Time to LDT (months) & 58 & $23-101$ & 41 & $20-99$ & 88 & 36-102 \\
\hline Extra hepatic disease & 69 & $80 \%$ & 42 & $78 \%$ & 27 & $84 \%$ \\
\hline \multicolumn{7}{|l|}{ Tumor volume liver: } \\
\hline$<25 \%$ & 34 & $40 \%$ & 26 & $48 \%$ & 8 & $25 \%$ \\
\hline $25-50 \%$ & 15 & $17 \%$ & 12 & $22 \%$ & 3 & $9 \%$ \\
\hline $50-75 \%$ & 12 & $14 \%$ & 4 & $7 \%$ & 8 & $25 \%$ \\
\hline$>75 \%$ & 19 & $22 \%$ & 9 & $17 \%$ & 10 & $31 \%$ \\
\hline$n / e$ & 6 & $7 \%$ & 3 & $6 \%$ & 3 & $9 \%$ \\
\hline Chromogranin $A^{*}$ & 1102 & $509-3537$ & 1105 & $532-3744$ & 1032 & $121-2616$ \\
\hline Functional symptoms & 53 & $62 \%$ & 40 & $74 \%$ & 13 & $41 \%$ \\
\hline
\end{tabular}




\begin{tabular}{|c|c|c|c|c|c|c|}
\hline (continuation) & All & $\%$ or IQR & TAE & $\%$ or IQR & SIRT & $\%$ or IQR \\
\hline \multicolumn{7}{|l|}{ (Prior) treatments: } \\
\hline SSA use & 71 & $83 \%$ & 49 & $91 \%$ & 22 & $69 \%$ \\
\hline Prior surgery & 69 & $80 \%$ & 54 & $100 \%$ & 15 & $47 \%$ \\
\hline Prior hepatic surgery/RFA & 6 & $7 \%$ & 0 & $0 \%$ & 6 & $19 \%$ \\
\hline Prior PRRT & 36 & $42 \%$ & 16 & $30 \%$ & 9 & $28 \%$ \\
\hline Prior MBIG therapy & 6 & $7 \%$ & 6 & $11 \%$ & 0 & $0 \%$ \\
\hline Prior IFa & 12 & $14 \%$ & 9 & $17 \%$ & 3 & $9 \%$ \\
\hline \multicolumn{7}{|l|}{ Laboratory values: } \\
\hline Bilirubin (umol/L) & 10 & $7-13$ & 10 & $6-13$ & $11^{* *}$ & $8-13$ \\
\hline ASAT (U/I) & 31 & $25-43$ & 32 & $25-44$ & $31^{* *}$ & $25-43$ \\
\hline ALAT (U/I) & 29 & $20-43$ & 29 & $20-44$ & $27^{* *}$ & $20-40$ \\
\hline \multicolumn{7}{|c|}{$\begin{array}{l}\text { * in } 78,49,29 \text { patients respectively; ** in } 30 \text { patients; TAE: transarterial embolization; SIRT: selective } \\
\text { internal radiation therapy; IQR: interquartile range; GEP: gastroenteropancreatic; NET: } \\
\text { neuroendocrine tumour; UP: unknown primary; p: pancreatic; LDT: liver directed therapy; n/e: not } \\
\text { evaluated; SSA: somatostatin analogue; RFA: radiofrequent ablation; MBIG: meta- } \\
\text { iodobenzylguanidine; IFa:interferon- } \alpha \text {; ASAT: aspartate aminotransferase; ALAT: alanine } \\
\text { aminotransferase }\end{array}$} \\
\hline
\end{tabular}

Table 2: Procedural characteristics

\begin{tabular}{|c|c|c|c|c|}
\hline \multirow[b]{2}{*}{ Characteristic } & \multicolumn{2}{|c|}{ TAE } & \multicolumn{2}{|c|}{ SIRT } \\
\hline & $\mathbf{n}$ & $\%$ or IQR & n or median & $\%$ or IQR \\
\hline Total procedures: & 90 & $\mathbf{n} / \mathbf{a}$ & 40 & n/a \\
\hline \multicolumn{5}{|l|}{ Extent of procedure: } \\
\hline Hemi liver/selected liversegment(s) & 48 & $89 \%$ & 19 & $59 \%$ \\
\hline Whole liver & 6 & $11 \%$ & 13 & $41 \%$ \\
\hline \multicolumn{5}{|l|}{ Frequency of procedure per patient: } \\
\hline One treatment & 25 & $91 \%$ & 25 & $91 \%$ \\
\hline Sequential treatments & 4 & $7 \%$ & 3 & $9 \%$ \\
\hline Multiple treatments & 1 & $2 \%$ & - & - \\
\hline Administered activity glass (MBq) & $\mathbf{n} / \mathbf{a}$ & $\mathbf{n} / \mathbf{a}$ & 3085 & $1960-4266$ \\
\hline Administered activity resin (MBq) & $\mathbf{n} / \mathbf{a}$ & $\mathbf{n} / \mathbf{a}$ & 1183 & 1008-2279 \\
\hline
\end{tabular}

TAE: transarterial embolization; SIRT: selective internal radiation therapy; n/a:not applicable

\section{- Toxicity}

Mild adverse events (CTCAE grade 1 and 2) occurred frequently in both treatment groups. Mild biochemical toxicity was similar in both groups, encountered up to 
$72 \%$ and $75 \%$ of the procedures in TAE and SIRT, respectively. Mild clinical toxicity differed in both groups, encountered up to $72 \%$ and $46 \%$ of the procedures in TAE and SIRT, respectively. Severe biochemical toxicity differed between the LDT. Severe (grade 3 or 4) biochemical toxicity, mainly transient elevated liver enzymes, occurred up to $47 \%$ and $13 \%$ of the procedures after TAE and SIRT, respectively. Severe clinical toxicity occurred rarely after LDT, up to $3 \%$ of the procedures in both treatment groups. Expressed in patients (instead of procedures) 46 patients (85\%) experienced severe biochemical toxicity after TAE, while five patients (16\%) experienced severe biochemical toxicity after SIRT. Severe clinical toxicity occurred in two patients (4\%) en one patient (3\%) undergoing TAE and SIRT, respectively (Table 4).

- Response

Clinical, biochemical and radiological response was evaluated during 3 months of follow-up after TAE and SIRT. In the entire cohort, the only significant difference in response regarded the reduction in clinical symptoms for patients treated with TAE $(82 \%)$ compared to SIRT $(42 \% \mathrm{P}=0.002)$. An overview of all response rates is shown in Table 5. Since functional symptoms occur more frequently in SI-NETs and this type of origin was overrepresented in the TAE cohort, a separate analysis was performed for SI-NETs and pNETs. In SI-NETs, TAE appeared to reduce functional symptoms more effectively compared to SIRT. Moreover, a trend was observed in the reduction of Chromogranin $\mathrm{A}$ in patients treated with TAE. This resulted in a significant higher combined response rate for TAE in SI-NETs. However, radiological response rates were equal for TAE and SIRT in SI-NETs. No difference was observed in the response rates of patients with a PNET, treated with either TAE or SIRT.

\section{- Survival}

No difference in overall survival was seen. Median overall survival was 33 months and 38 months for TAE vs. SIRT, respectively. Although median overall survival in SINETs was 33 months compared to 66 months for TAE vs. SIRT, this difference was not statistically significant. For patients with a pNET, a trend towards a better survival was seen in patients treated with SIRT. Survival curves are shown in the supplement. 
Part II | Chapter 7

Table 3: Toxicity per procedure

Biochemical toxicity*

\begin{tabular}{|c|c|c|c|c|c|c|c|c|c|c|c|c|}
\hline \multirow[b]{3}{*}{ Gr 1} & \multicolumn{4}{|c|}{ Bilirubin } & \multicolumn{4}{|c|}{$\underline{\text { ASAT }}$} & \multicolumn{4}{|c|}{ ALAT } \\
\hline & \multicolumn{2}{|c|}{ TAE } & \multicolumn{2}{|c|}{ SIRT } & \multicolumn{2}{|c|}{ TAE } & \multicolumn{2}{|c|}{ SIRT } & \multicolumn{2}{|c|}{ TAE } & \multicolumn{2}{|c|}{ SIRT } \\
\hline & 21 & $24 \%$ & 9 & $23 \%$ & 10 & $11 \%$ & 24 & $60 \%$ & 26 & $29 \%$ & 19 & $48 \%$ \\
\hline Gr 2 & 9 & $10 \%$ & - & - & 7 & $8 \%$ & 6 & $15 \%$ & 21 & $23 \%$ & 2 & $5 \%$ \\
\hline Gr 3 & 19 & $21 \%$ & 5 & $13 \%$ & 42 & $47 \%$ & 1 & $3 \%$ & 34 & $38 \%$ & 1 & $3 \%$ \\
\hline \multirow[t]{4}{*}{ Gr 4} & 1 & $1 \%$ & 1 & $3 \%$ & 30 & $33 \%$ & 1 & $3 \%$ & 6 & $7 \%$ & - & - \\
\hline & \multicolumn{12}{|c|}{ Clinical toxicity } \\
\hline & \multicolumn{4}{|c|}{ Pain } & \multicolumn{4}{|c|}{ Fever } & \multicolumn{4}{|c|}{ Nausea } \\
\hline & \multicolumn{2}{|c|}{ TAE } & \multicolumn{2}{|c|}{ SIRT } & \multicolumn{2}{|c|}{ TAE } & \multicolumn{2}{|c|}{ SIRT } & \multicolumn{2}{|c|}{ TAE } & \multicolumn{2}{|c|}{ SIRT } \\
\hline Gr 1 & 5 & $6 \%$ & 6 & $15 \%$ & 33 & $37 \%$ & 4 & $10 \%$ & 3 & $3 \%$ & 3 & $8 \%$ \\
\hline Gr 2 & 65 & $72 \%$ & 12 & $30 \%$ & 34 & $38 \%$ & 3 & $8 \%$ & 46 & $51 \%$ & 8 & $21 \%$ \\
\hline Gr 3 & - & - & - & - & 3 & $3 \%$ & - & - & - & - & - & - \\
\hline \multirow[t]{3}{*}{ Gr 4} & - & - & - & - & - & - & - & - & - & - & - & - \\
\hline & \multicolumn{4}{|c|}{ Vomiting } & \multicolumn{4}{|c|}{ Malaise } & \multicolumn{4}{|c|}{ Delerium } \\
\hline & \multicolumn{2}{|c|}{ TAE } & \multicolumn{2}{|c|}{ SIRT } & \multicolumn{2}{|c|}{ TAE } & \multicolumn{2}{|c|}{ SIRT } & \multicolumn{2}{|c|}{ TAE } & SI & \\
\hline Gr 1 & 30 & $33 \%$ & 3 & $8 \%$ & 9 & $10 \%$ & 7 & $18 \%$ & 2 & $2 \%$ & 1 & $3 \%$ \\
\hline Gr 2 & 2 & $2 \%$ & 2 & $5 \%$ & 3 & $3 \%$ & 2 & $5 \%$ & 4 & $4 \%$ & - & - \\
\hline Gr 3 & 1 & $1 \%$ & - & - & - & - & - & - & - & - & - & - \\
\hline Gr 4 & - & - & - & - & - & - & - & - & - & - & - & - \\
\hline & & leural & fus & & & lepatic & disea & & & & & \\
\hline & & & & & & & & & TAE & & & \\
\hline Gr 1 & 9 & $10 \%$ & 2 & $3 \%$ & 4 & $4 \%$ & 1 & $3 \%$ & $\mathrm{n} / \mathrm{a}$ & $\mathrm{n} / \mathrm{a}$ & - & - \\
\hline Gr 2 & 10 & $11 \%$ & - & - & - & - & - & - & $\mathrm{n} / \mathrm{a}$ & $\mathrm{n} / \mathrm{a}$ & - & - \\
\hline Gr 3 & - & - & - & - & 1 & $1 \%$ & - & - & $\mathrm{n} / \mathrm{a}$ & $\mathrm{n} / \mathrm{a}$ & 1 & $3 \%$ \\
\hline Gr 4 & - & - & - & - & - & - & - & - & $\mathrm{n} / \mathrm{a}$ & $\mathrm{n} / \mathrm{a}$ & - & - \\
\hline
\end{tabular}

Table 4: Patients with severe toxicity during follow-up

\begin{tabular}{lcrrr} 
& \multicolumn{2}{c}{ TAE } & \multicolumn{2}{c}{ SIRT } \\
\hline \hline Biochemical toxicity & $\mathbf{4 6}$ & $\mathbf{8 5 \%}$ & $\mathbf{5}$ & $\mathbf{1 6 \%}$ \\
Clinical toxicity & $\mathbf{2}$ & $\mathbf{4} \%$ & $\mathbf{1}$ & $\mathbf{3 \%}$ \\
TAE: transarterial embolization; SIRT: selective internal radiation therapy; FU: follow-up &
\end{tabular}




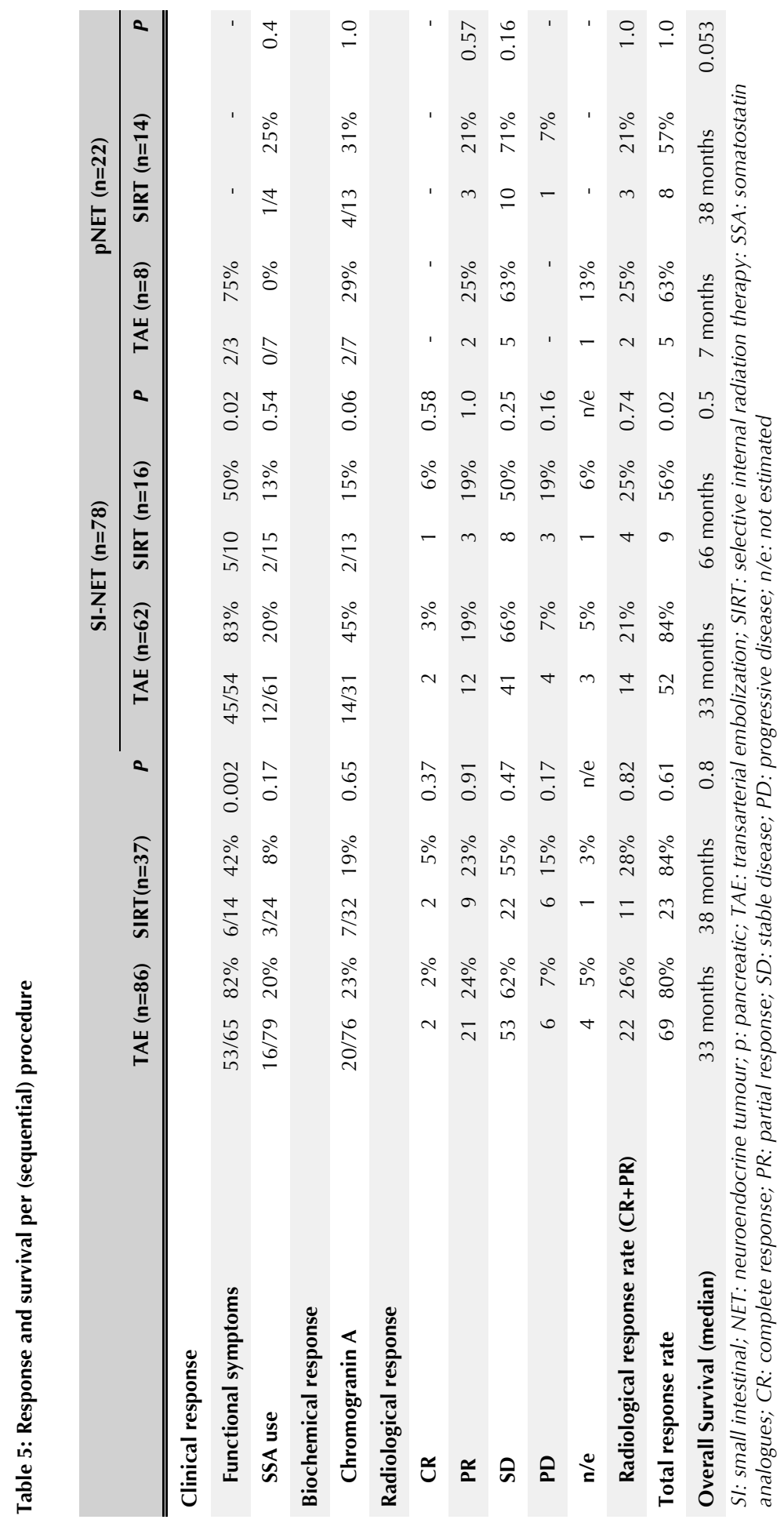




\section{Discussion}

This study is, to our knowledge, the first comparative study of TAE and SIRT with regard to toxicity, response and survival. Furthermore, a subgroup analysis was performed for SI-NET and pNET, the most prevalent location of a primary NET. Our study showed that both TAE and SIRT are safe and effective treatment modalities for patients with liver dominant $\mathrm{mNET}$, although they displayed different toxicity profiles. Overall, the total response rates did not differ, but functional symptoms in SI-NET seemed to decrease more often after TAE compared to SIRT. No differences in survival rates were observed.

The treatment of mNET remains a challenge. ${ }^{5,6,15,18}$ Compared to other malignancies, only limited treatment options are available for this heterogeneous patient population. In NET, LM are the most prevalent metastases which results in a significant disease burden. Optimal treatment of LM improves quality of life and clinical outcome. At present, the optimal intra-arterial therapy for NET LM has been scarcely investigated, especially in case of comparative research on SIRT and TAE.

To our knowledge, only two other studies have compared TAE with SIRT. Elf et al. prospectively investigated TAE and SIRT in eleven patients. ${ }^{19}$ They showed an equal biochemical response and radiological response in $\mathrm{mNET}$. However, the small sample size is a major limitation of this study. Chen et al. retrospectively identified a cohort of 155 patients. ${ }^{20}$ They identified prognostic factors and analysed survival in patients with NET undergoing embolotherapies. A significantly lower survival rate was seen in patients treated with SIRT, but adjusted by a propensity weight factor, this effect disappeared. Tumour grade was identified as a prognostic factor. Yet, tumour grade was unequally distributed between the treatment groups. Also, the primary site of origin was recognized as a prognostic factor, but no subgroup analyses were performed. Therefore, the results of this study are susceptible to confounding.

Clinically relevant severe adverse events were uncommon after TAE and SIRT. Furthermore, the reported toxicity rates in our cohort are corroborated by other studies. ${ }^{21,22}$ Hence, TAE and SIRT can be considered as safe treatment options. However, differences in toxicity between the treatments were seen. Mild clinical toxicity occurred more frequently in patients treated with TAE (72\% compared to $46 \%$ in patients treated with SIRT). Additionally, in patients treated with TAE a higher 
rate of severe biochemical toxicity was seen $(47 \%$ and $13 \%$ after TAE and SIRT, respectively). Nevertheless, the severe toxicity consisted mainly of transient elevated liver enzymes and required no additional treatment or deviation from treatment regimes.

After TAE functional symptoms significantly decreased in the entire cohort and specifically in patients with a SI-NET, whilst this effect was not observed in pNETs. Since SI-NETs comprise the largest group and functional symptoms were more prevalent in SI-NETs, this probably impacted the results of the entire TAE cohort. Furthermore, the presence of functional symptoms was extracted from patient's charts and no standardized method for registration or questionnaire was performed. Therefore, the more favourable performance of TAE in reducing functional symptoms should therefore be interpreted with caution.

Of all response analyses, radiological response is most likely to have an impact on survival. No difference in radiological response and subsequently median survival was seen. Although a trend for an improved survival was suggested for patients with a pNET treated with SIRT. However due to the small sample size, this result should be confirmed in a larger patient population.

The different toxicity and response profiles might influence which treatment prefers, taking into consideration individual patients' characteristics or indication to undergo TAE or SIRT (e.g. origin of NET, relieving functional symptoms or progressive disease). Furthermore, mild clinical toxicity might have a bigger impact on patient's quality of life compared to severe transient elevated liver enzymes.

Other frequently used treatment modalities in LM of NETs are SSAs and Peptide Receptor Radionuclide Therapy (PRRT). SSA's offer symptom control and the CLARINET trial has shown its anti-proliferative effect. ${ }^{23}$ In recent years, PRRT has been a major advancement in the treatment of mNETs by significantly improving progression free survival and OS. ${ }^{24}$ Studies have shown LDTs do not prevent PRRT in the future or vice versa. ${ }^{25}$ Moreover, previous (liver) surgery is not a contra-indication for LDT. ${ }^{26}$ Therefore, LDT remains a suitable and relevant treatment option in the arena of treatment modalities in liver dominant disease in NET.

Several limitations of our study should be taken into consideration. Firstly, a retrospective cohort study was performed. This study design is prone for incomplete 
data retrieval. Preferably more laboratory values would have been collected and a standardized registration of functional symptoms would have been performed. Particularly to compare treatments a standardized prospective design, such as a randomized controlled trial, would have been preferred. Nevertheless, patients received TAE or SIRT depending on primary treatment center, which limits bias in treatment decision-making despite of the retrospective design of the study. Secondly, the patient population was highly heterogeneous, which is an inherent limitation of NET research. We attempted to correct for the most important variances in patient characteristics by including only low-grade NETs and performing subgroup analyses. Furthermore, the response evaluation after SIRT did not include actual delivered dose by post-treatment evaluation. Of note, improvement of dosimetry could improve response after SIRT. ${ }^{27}$ Also in the response and toxicity analyses of SIRT, the use of resin as well as glass particles were combined. Preferably, a separate analysis was performed for SIRT using glass or resin particles, since they differ in size, number if microspheres and activity per microsphere. ${ }^{28-30}$ However, the small numbers did not allow separate analysis.

Since TAE and SIRT are both safe and effective, individual patient characteristics could be taken into account when deciding on a treatment regime, such as the origin of the NET, presence of functional symptoms or the different toxicity profiles in the context of the patient's co-morbidities. Future studies should aim at sequential and combined therapies in determining the optimal treatment strategy in mNET. Quality of life studies should be included and a cost-effectiveness analysis is warranted to compare the costs and health gains of TAE and SIRT.

\section{Conclusion}

Both TAE and SIRT are safe and effective treatment modalities for patients with liver dominant $\mathrm{mNET}$, with different toxicity and response profiles. Overall, the total response rates did not differ, but functional symptoms might decrease more often after TAE compared to SIRT, especially in SI-NETs. No differences in survival rates were observed, but a trend was seen towards better survival after SIRT in patients with LM of a pNET. 


\section{References}

1. Yao JC, Hassan M, Phan A, et al. One hundred years after "carcinoid": epidemiology of and prognostic factors for neuroendocrine tumors in 35,825 cases in the United States. J Clin Oncol. 2008;26(18):3063-3072

2. Hallet J, Law $\mathrm{CH}$, Cukier $\mathrm{M}$, et al. Exploring the rising incidence of neuroendocrine tumors: a population- based analysis of epidemiology, metastatic presentation, and outcomes. Cancer. 2015;121(4):589-597.

3. Modlin IM, Lye KD, Kidd M. A 5-decade analysis of 13,715 carcinoid tumors. Cancer. 2003;97(4):934-959.

4. Rossi RE, Massironi S, Spampatti MP, et al. Treatment of liver metastases in patients with digestive neuroendocrine tumors. J Gastrointest Surg. 2012;16:1981-1992

5. Frilling A, Clift AK. Therapeutic strategies for neuroendocrine liver metastases. Cancer. 2015;121(8):1172-86.

6. Chamberlain RS, Canes D, Brown KT, et al. Hepatic neuroendocrine metastases: does intervention alter outcomes? J Am Coll Surgeons. 2000;190;432-45

7. Quaedvlieg PF, Visser O, Lammers CB et al. Epidemiology and survival in patients with carcinoid disease in The Netherlands. An epidemiological study with 2391 patients. Ann Oncol 2001;12;1295-300

8. Saxena A, Chua TC, Perara M, et al. Surgical resection of hepatic metastases from neuroendocrine neoplasms: a systematic review. Surg Oncol. 2012;21:e131-e141

9. Fairweather M, Swanson R, et al. Management of Neuroendocrine Tumor Liver Metastases: Long-Term Outcomes and Prognostic Factors from a Large Prospective Database. Ann Surg Oncol. Published online: 16-03-2017.

10. de Baere T, Deschamps F, Tselikas L, et al. GEP-NETS update: Interventional radiology: role in the treatment of liver metastases from GEP-NETs. Eur J Endocrinol. 2015;172(4):R151-66.

11. Kennedy A, Bester L, Salem R, et al. Role of hepatic intra-arterial therapies in metastatic neuroendocrine tumours (NET): guidelines from the NET-liver-Metastases Consensus Conference. HPB (2015) 17:29-39

12. Lewis MA, Hobday TJ. Treatment of Neuroendocrine Tumor Liver Metastases. International Journal of Hepatology. 2012;2012:1-12.

13. Pavel M, Baudin E, Couvelard A, et al. ENETS Consensus Guide- lines for the Management of Patients with Liver and Other Distant Metastases from Neuroendocrine Neoplasms of Foregut, Mid- gut, Hindgut, and Unknown Primary. Neuroendocrinology 2012;95:157-176 
14. Devcic Z, Rosenberg J, Braat AJA, et al. The Efficacy of Hepatic 90Y Resin Radioembolization for Metastatic Neuroendocrine Tumors: A Meta-Analysis. J Nucl Med. 2014; 55: 1404-1410.

15. National Cancer Institute NloH. Common terminology criteria for adverse events v4.03.2010.

16. Braat MN, van Erpecum KJ, Zonnenberg BA, et al. Radioembolization-induced liver disease: a systematic review. Eur J Gastroenterol Hepatol. 2017 Feb;29(2):144-152.

17. Eisenhauer EA, Therasse P, Bogaerts J, et al. New response evaluation criteria in solid tumours: revised RECIST guideline (version 1.1). Eur J Cancer. 2009;45(2):228-47.

18. Cavalcoli F, Rausa E, Conte D, et al. Is there still a role for the hepatic locoregional treatment of metastatic neuroendocrine tumors in the era of systemic targeted therapies? World Journal of Gastroenterology. 2017;23(15):2640.

19. Elf AK, Andersson M, Henrikson O, et al. Radioembolization Versus Bland Embolization for Hepatic Metastases from Small Intestinal Neuroendocrine Tumors: Short-Term Results of a Randomized Clinical Trial. World J Surg. 2018;42(2):506-13.

20. Chen JX, Rose S, White SB, et al. Embolotherapy for Neuroendocrine Tumor Liver Metastases: Prognostic Factors for Hepatic Progression-Free Survival and Overall Survival. CardioVascular and Interventional Radiology. 2016;40(1):69-80.

21. Gupta S. Intra-arterial liver-directed therapies for neuroendocrine hepatic metastases. Semin Intervent Radiol. 2013;30(1):28-38.

22. Jia Z, Wang W, Yttrium-90 radioembolization for unresectable metastatic neuroendocrine liver tumor: A systematic review. Eur J Radiol.

23. Caplin ME, Pavel M, Ćwikła JB, et al. CLARINET Investigators. Lanreotide in metastatic enteropancreatic neuroendocrine tumors. N Engl J Med. 2014 Jul 17;371(3):224-33. 2018;100:23-9.

24. Strosberg JR, Wolin EE, Chasen BA, et al. First update on overall survival, progressionfree survival, and health-related time-to-deterioration quality of life from the NETTER1 study: 177Lu-Dotatate vs. high dose octreotide in progressive midgut neuroendocrine tumors. JCO. 2018;36(15 supplemental):4099.

25. Braat AJAT, Ahmadzadehfar H, Kappadath SC, et al. Radioembolization with (90) $\mathrm{Y}$ Resin Microspheres of Neuroendocrine Liver Metastases After Initial Peptide Receptor Radionuclide Therapy. Cardiovasc Intervent Radiol. 2019 Oct 23.

26. Samim M, van Veenendaal LM, Braat MNGJA, et al. Recommendations for $r$ adioembolisation after liver surgery using yttrium-90 resin microspheres based on a survey of an international expert panel. Eur Radiol. 2017 Dec;27(12):4923-4930.

27. Chansanti O, Jahangiri Y, Matsui Y, et al. Tumor Dose Response in Yttrium-90 Resin Microsphere Embolization for Neuroendocrine Liver Metastases: A Tumor-Specific 
Analysis with Dose Estimation Using SPECT-CT. Journal of Vascular and Interventional Radiology. 2017;28(11):1528-35.

28. James T, Hill J, Fahrbach T et al. Differences in Radiation Activity Between Glass and Resin 90Y Microspheres in Treating Unresectable Hepatic Cancer. Health Phys. 2017 Mar;112(3):300-304.

29. Nezami N, Kokabi N, Camacho JC, et al. (90)Y radioembolization dosimetry using a simple semi-quantitative method in intrahepatic cholangiocarcinoma: Glass versus resin microspheres. Nucl Med Biol. 2018 Apr;59:22-28.

30. Van Der Gucht A, Jreige M, Denys A, et al. Resin Versus Glass Microspheres for (90)Y Transarterial Radioembolization: Comparing Survival in Unresectable Hepatocellular Carcinoma Using Pretreatment Partition Model Dosimetry. J Nucl Med. 2017 Aug;58(8):1334-1340. 
Part II | Chapter 7

\section{Supplementary Graphs S1: Survival curves}
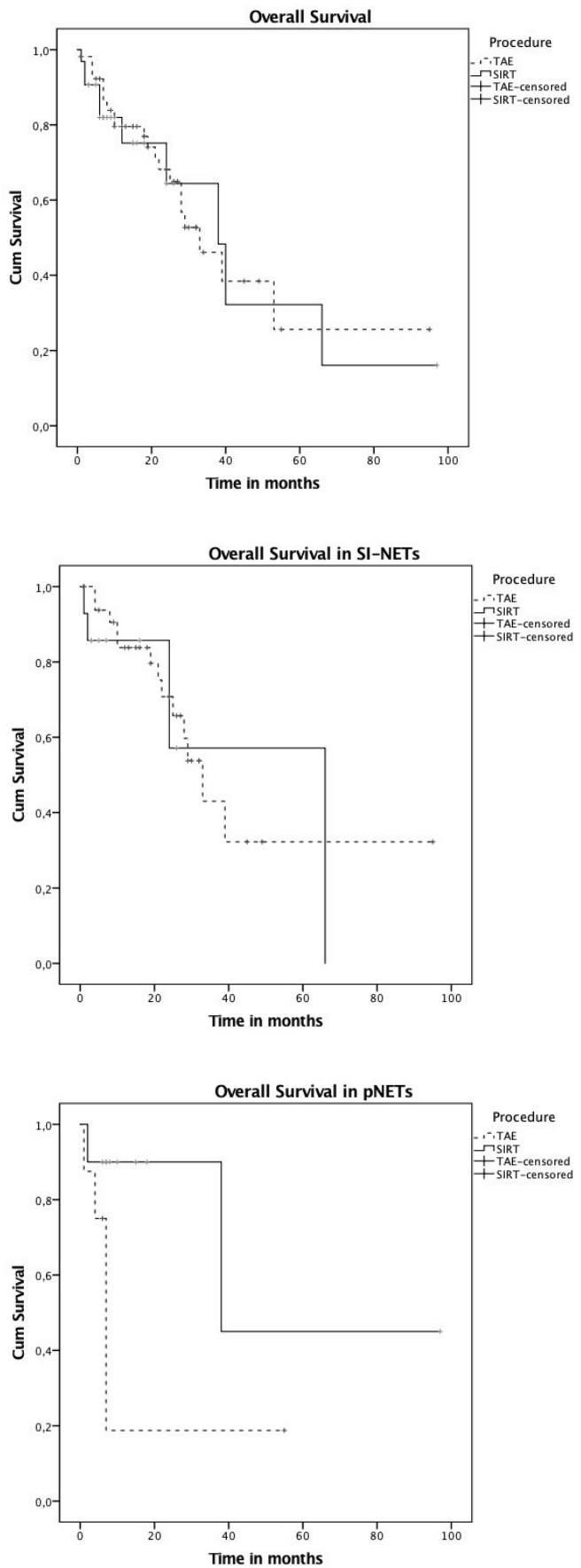



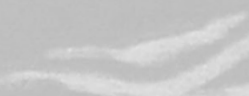

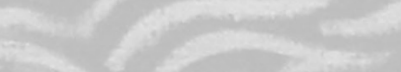

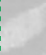

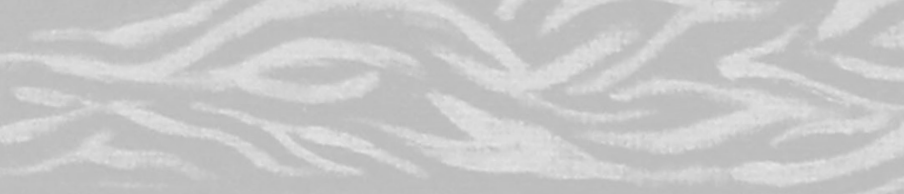




\section{Chapter 8}

\section{Efficacy of isolated limb perfusion in patients with Merkel cell carcinoma: a multicenter experience}

L.M. van Veenendaal*, M.F. Madu*, M.E.T. Tesselaar, C. Verhoef, D.J. Grünhagen, A.C.J. van Akkooi, * both authors contributed equally

European Journal of Surgical Oncology, 2017 


\begin{abstract}

\section{Background}

Merkel cell carcinoma (MCC) is a rare and potentially aggressive neuroendocrine tumour of the skin, with a propensity for loco regional metastases. In two expert referral centers, isolated limb perfusion (ILP) is used to obtain loco regional control in selected loco regionally advanced MCC patients.
\end{abstract}

\title{
Aim
}

This study describes our experience and clinical outcomes for patients with MCC treated with ILP.

\section{Patients and Methods}

Patients who underwent ILP for MCC were analysed. ILP was performed with melphalan and tumour necrosis factor (TNF) combination therapy. Depending on the institution, either a norm thermic or a hyperthermia temperature regimen was used. Baseline characteristics, toxicity data, loco regional progression-free survival (LPFS) and overall survival (OS) were assessed.

\section{Results}

Four males and 6 females with a median age of 78 years (IQR 61-84 years) were included. Four patients underwent ILP for upper extremity disease and 6 for lower extremity disease. All patients received combination therapy with Melphalan and TNF, one patient with the addition of interferon-gamma. No signs of systemic toxicity were present post-ILP. Severe loco regional toxicity (compartment syndrome) occurred in one patient and another elderly patient with extensive atherosclerosis had to undergo trans femoral amputation due to critical ischemia. Eight patients could be included for response evaluation. The overall response rate (ORR) was $87.5 \%$ with a complete response (CR) rate of $62.5 \%$. Two long-term responses of 53 months and 71 months were observed. Median LPFS was 5 months and median OS was 54 months.

\section{Conclusion}

ILP shows a high CR rate that can be durable. Therefore, ILP should be considered an effective treatment modality for locally advanced MCC. 


\section{Introduction}

Merkel cell carcinoma (MCC) is a rare and potentially aggressive neuroendocrine tumour of the skin. It affects mostly elderly Caucasian patients, with a slight overrepresentation of males. The head and neck and extremities are predominant localizations, supporting the assumption that ultraviolet exposure increases the risk for MCC. ${ }^{1,2}$ Other risk factors are immunosuppression and Merkel cell Polyomavirus (MCPyV). ${ }^{3} \mathrm{MCC}$ is a malignant disease with a high metastatic potential. At diagnosis approximately $35-40 \%$ of patients have nodal involvement. ${ }^{4-6}$ Loco regional recurrence is seen in $26-60 \%$ of the patients, often within 2 years. ${ }^{7,8}$ This can present as true local recurrence or in-transit metastasis (ITM); discrete cutaneous or subcutaneous intralymphatic metastases. Survival of patients with loco regionally recurrent disease is poor, only $39 \%$ after 3 years. ${ }^{9}$

Therapeutic options consist of surgery and radiotherapy, chemotherapy is only indicated for patient with distant metastases. ${ }^{10-13}$ While loco regional disease is generally managed with surgery and/or radiotherapy, there is no standard treatment for loco regionally advanced disease. ${ }^{14}$ In patients with loco regionally advanced melanoma, isolated limb perfusion (ILP) of the extremities proved to be safe and

effective. ${ }^{15,16}$ However, its role in patients with MCC remains unclear. Several small series showed a high complete response rate in patients with locally advanced MCC. ${ }^{15,17-21}$ In our two melanoma and neuroendocrine expert-centers in the Netherlands, ILP is performed for patients with melanoma as well as MCC. We aimed to describe our experience with ILP in MCC patients.

\section{Patients and methods}

This study was performed in accordance with institutional ethical guidelines. All MCC patients who underwent an ILP in the Netherlands Cancer Institute $(\mathrm{NCI})$ and the Erasmus MC Cancer Institute (EMC) until October 2016 were included in this retrospective cohort study. Patient and tumour characteristics, treatment and followup data were obtained from patient records and collected in a database for this study. 
- ILP indication and technique

ILP was performed in patients with locally advanced or unresectable MCC and multiple or quickly recurring MCC in-transit metastases. The technique for ILP in melanoma has been described in detail previously. ${ }^{22,23}$ The same technique is used for ILP in MCC. In short, the patient is placed under general anaesthesia, after which the main artery and vein of the extremity are surgically accessed. The patient is heparinized and after a minimum of 2 min the artery and vein are clamped, cannulated and connected to an oxygenated extracorporeal circuit. An inflatable tourniquet ensures compression of collateral vessels to prevent systemic leakage of perfusate. Inadvertent systemic leakage is monitored by a precordial gamma probe, which detects technetium-labelled albumin added to the perfusate. Temperature probes are placed subcutaneously and in a muscle compartment. When the temperature of the extremity has reached $37 \mathrm{C}$ or $38 \mathrm{C}$, depending on the temperature regimen used, TNF is added to the perfusion circuit. The dose of TNF used was 2-4 $\mathrm{mg}$ and dependent on calculated extremity volume. After $30 \mathrm{~min}$, melphalan in a dose of $10 \mathrm{mg} / \mathrm{L}$ extremity volume is added to the circuit. During the procedure the temperature of the extremity is kept between $37 \mathrm{C}$ and $38 \mathrm{C}$ for normothermic ILP and between 38C and 40C for hyperthermia ILP. In the $\mathrm{NCI}$ both normothermic and hyperthermic ILP's were performed for MCC during the study period, in the EMC solely hyperthermic ILP's. One hour after the addition of melphalan, the extremity is flushed with saline and the continuity of the circulation is restored. Perfusion characteristics such as temperature, flow rate and amount of systemic leakage are recorded during the procedure.

- Response rates, toxicity and complications

Response to ILP was scored according to the RECIST criteria and defined as the best overall response reached before eventual progression. ${ }^{24}$ Local toxicity was scored according to the Wieberdink scale. ${ }^{25}$ Complications were scored according to the Clavien/Dindo classification. ${ }^{26}$ Patients were staged according to the AJCC 7 th edition. $^{27}$

- Follow-up

Follow-up took place 6 weeks after discharge from the hospital, every 3 months for the first year, every 4 months for the second year, every six months for year 3-5 and 
yearly thereafter. Patients underwent physical examination during each visit. When patients presented with symptoms, they were restaged with imaging (CT or PET/CT). Relapses were scored as loco regional relapse, regional nodal relapse or distant relapse. Loco regional relapse was defined as local recurrence, satellite metastasis or an in-transit metastasis. Regional relapse was defined as regional nodal recurrence in the draining lymph nodal basin. Distant relapse was defined as subcutaneous or nodal relapse beyond the regional nodal basin or haematogenous relapse.

- Statistical analysis

The time to ILP was calculated from the date of primary MCC diagnosis to the ILP date. Survival curves were generated with the Kaplan-Meier method. MCC-specific survival (MSS) was calculated from the date of the ILP to the date of death caused by MCC or last follow-up. Loco regional progression-free survival (LPFS) was calculated from the date of the ILP to the date of the first loco regional relapse, death or last follow-up. All statistical analyses were performed using SPSS version 22.0 or STATA version 13.

\section{Results}

Ten patients divided evenly over our institutions, underwent ILP for MCC. Patient characteristics are shown in Table 1. Four patients were male (40\%). The median age at the time of ILP was 78 years (IQR 61-84 months). Four patients underwent ILP for upper extremity disease and 6 for lower extremity disease. The median time from diagnosis of the primary tumour until the ILP was 7 months (IQR 2-22 months). The indication for ILP was the presence of multiple unresectable in-transit metastases in 5 cases and bulky, solitary locally advanced disease in the other 5 cases. Four patients had concomitant clinically detectable nodal disease (AJCC stage IIIB) either before or during ILP, while six patients had no nodal involvement (stage II).

\section{- Treatment characteristics}

Treatment characteristics are shown in Table 2. A detailed overview of patients, treatment and outcomes is shown in Table 3. Four patients underwent a femoral perfusion, two patients underwent iliac perfusion, three patients underwent axillary perfusion and one patient underwent brachial perfusion. Three patients underwent a 
lymph node dissection (LND) during the ILP, either as part of obtaining vascular access $(n=1)$ or because of proven lymph node metastasis $(n=2)$. The median flow rate was $250 \mathrm{ml} / \mathrm{min}$ (IQR 190-328). All patients received combination therapy with melphalan and TNF, with a median melphalan dose of $65 \mathrm{mg}$ (IQR 44-73) and a median TNF dose of $3 \mathrm{mg}$ (IQR 2-3). One patient received $0.2 \mathrm{mg}$ interferon-gamma in addition to melphalan and TNF in a clinical trial setting. Four ILP's were normothermic and six were hyperthermic.

Table 1: Baseline characteristics

\begin{tabular}{lrr} 
Characteristic & $\begin{array}{r}\text { n or } \\
\text { median }\end{array}$ & or \\
& IQR \\
\hline \hline
\end{tabular}

\section{Gender:}

\begin{tabular}{|lrr|}
\hline \multicolumn{1}{|l|}{ Female } & 6 & 60 \\
\hline Male & 4 & 40 \\
\hline Age & 78 & $61-84$ \\
\hline $\begin{array}{l}\text { Indication for ILP: } \\
\quad \text { In-transit }\end{array}$ & 5 & 50 \\
\hline Locally advanced & 5 & 50 \\
\hline Time to ILP (months) & 7 & $2-22$ \\
\hline Limb: & & \\
\hline Arm & 4 & 40 \\
\hline Leg & 6 & 60 \\
\hline Nodal status: & & \\
\hline Negative & 6 & 60 \\
\hline Positive & 4 & 40 \\
\hline
\end{tabular}

\section{Prior LND:}

$\begin{array}{lll}\text { No } & 8 & 80 \\ \text { Yes } & 2 & 20\end{array}$

Prior RT:

$\begin{array}{lrr}\text { No } & 10 & 100 \\ \text { Yes } & 0 & 0\end{array}$

AJCC stage at perfusion:

$\begin{array}{lll}\text { II } & 6 & 60 \\ \text { IIIB } & 4 & 40\end{array}$

IQR: interquartile range; ILP: Isolated Limb Perfusion; RT: radiotherapy; LND: lymph node dissection

\section{Table 2: Treatment characteristics}

\begin{tabular}{lrr} 
Characteristic & $\begin{array}{r}\text { n or } \\
\text { median }\end{array}$ & $\begin{array}{r}\% \text { or } \\
\text { IQR }\end{array}$ \\
\hline \hline
\end{tabular}

\begin{tabular}{lcc}
\hline \hline ILP type: & & \\
Femoral & 4 & 40 \\
Iliac & 2 & 20 \\
Axillary & 3 & 30 \\
Brachial & 1 & 10
\end{tabular}

Temperature $\left({ }^{\circ} \mathrm{C}\right)$ :

\begin{tabular}{|lrr|}
\hline \multicolumn{1}{|l}{ Normothermic } & 4 & 40 \\
\hline \multicolumn{1}{|c|}{ Hyperthermic } & 6 & 60 \\
\hline TNF alpha dose (mg) & 3 & $2-3$ \\
\hline Melphalan dose (mg) & 65 & $44-73$ \\
\hline Agent: & 9 & 90 \\
$\quad$ Melphalan + TNF & 1 & 10 \\
\hline $\begin{array}{l}\text { Melphalan + TNF + IFy } \\
\text { Flow rate (ml/min) }\end{array}$ & 250 & $190-328$ \\
\hline
\end{tabular}

Systemic leakage:

$$
<5 \%
$$

LND during ILP?:

\begin{tabular}{|c|c|c|}
\hline LND & 3 & 30 \\
\hline No LND / unknown & 7 & 70 \\
\hline \multicolumn{3}{|c|}{$\begin{array}{l}\text { ILP: isolated limb perfusion; IQR: interquartile } \\
\text { range; TNF: tumor necrosis factor; Ify: interferon- } \\
\text { gamma; LND: lymph node dissection }\end{array}$} \\
\hline
\end{tabular}


- Toxicity and complications

Four patients developed a Wieberdink II reaction (slight erythema and/or oedema) and two patients developed a Wieberdink III reaction (considerable erythema and/or oedema with blistering). The local toxicity grade was unknown in two patients, but no compartment syndromes or reactions necessitating amputation were reported, so these patients were scored as Wieberdink < III. There was one patient with a compartment syndrome of the forearm after ILP, requiring fasciotomy (Wieberdink IV). One suffered Wieberdink $V$ toxicity, necessitating amputation. This female patient was 83 years old at the time of ILP and developed critical ischemia of the lower extremity. Despite timely trans femoral amputation, rhabdomyolysis with progressive renal failure caused metabolic acidosis, which led to death. Pathological examination of the amputated extremity showed extensive atherosclerosis. Systemic leakage of perfusate $>5 \%$ did not occur and there were no signs of systemic toxicity. Three patients suffered a complication not related to local toxicity. The first patient had a history of angina and atrial fibrillation. This patient developed postoperative atrial fibrillation for which intravenous antiarrhythmics were indicated, followed by cardiac decompensation that required treatment on the intensive care unit. The second patient underwent a reoperation due to arterial bleeding from a torn collateral vessel. Afterwards a superficial wound infection occurred that could be managed conservatively. The last patient (60 years old during ILP) with a history of severe congestive heart failure developed postoperative cardiac failure and progressive renal function impairment, leading to death shortly after the ILP.

\section{- Response and survival}

Eight patients could be included for response evaluation and survival analysis. The overall response rate (ORR) was $7 / 8$, with a complete response (CR) rate of 5/8 and a partial response (PR) rate of 2/8. The median follow-up was 27 months (IQR 17-54 months). No patients required amputation for unresectable progressive loco regional disease after perfusion, although as stated before, one required amputation due to toxicity. The median LPFS was 5 months, 6 months for patients with a CR and 3 months for patients with a PR or stable disease (SD). However, this difference was not significant $(P=0.270)$. The median distant metastasis-free survival (DMFS) was not reached. The median overall survival (OS) was 54 months. 


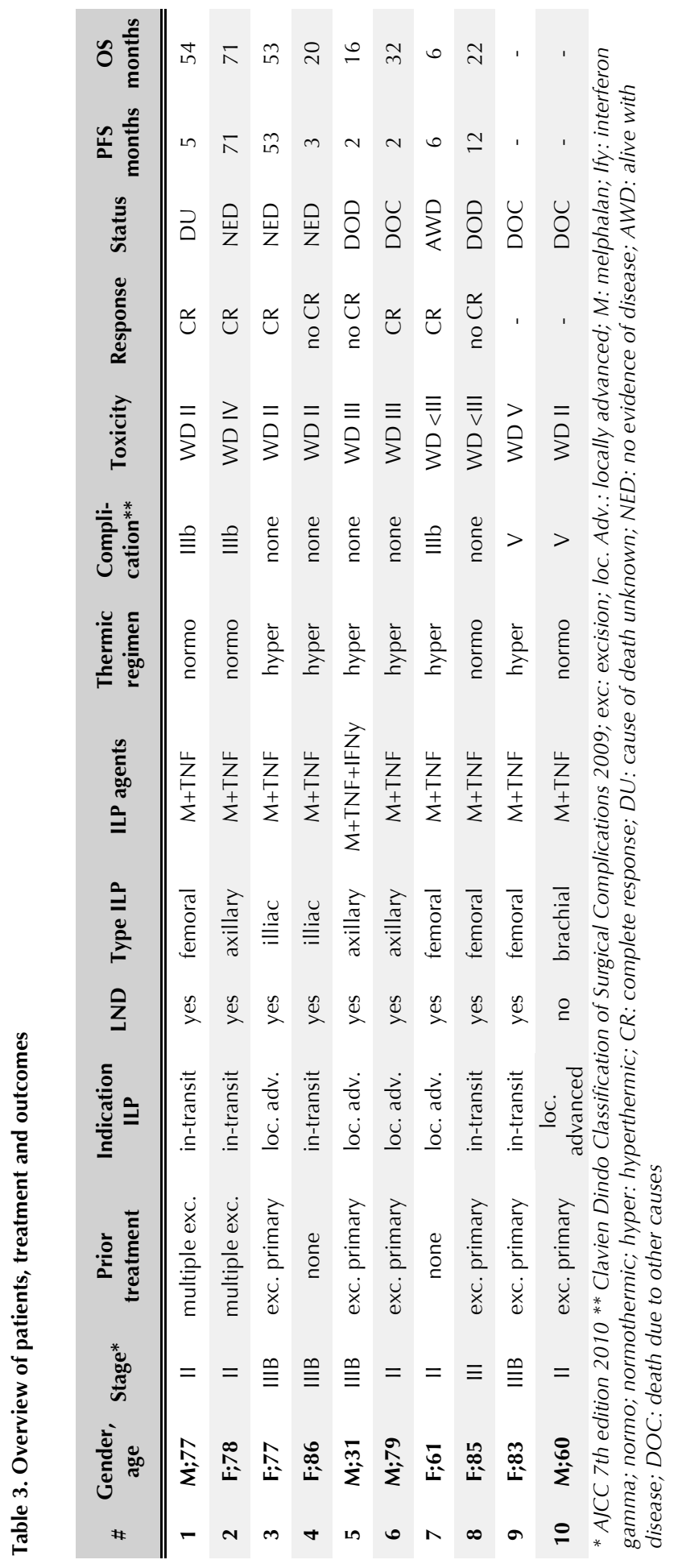


- Characteristics of durable responders

Two out of eight patients have an on-going CR after ILP without signs of out-of-field progression. The first (female) patient underwent ILP at 78 years, six years after primary MCC diagnosis. ILP was indicated because of quickly recurring multiple (>6) subcutaneous in-transit metastases (largest $15 \mathrm{~mm}$ in size) on the right forearm and upper arm. PET/CT staging did not show any other disease localizations. After normothermic axillary ILP with $2 \mathrm{mg}$ TNF and $45 \mathrm{mg}$ melphalan, the patient first suffered a compartment syndrome, for which fasciotomy of the volar and dorsal muscle compartment was performed. After three months, a CR was observed, which has been on-going for 6 years (71 months). The second (female) patient was 77 years old during ILP. The primary MCC on her left upper leg had been excised only six months earlier, together with sentinel node biopsy, which showed metastasis in two sentinel nodes. A superficial inguinal dissection was performed, which did not yield additional positive lymph nodes. In the following months, multiple bulky in-transit metastases developed and ILP was planned. Preoperative PET/CT staging did not show any distant metastases. Normothermic ILP was performed with $3 \mathrm{mg}$ TNF and $70 \mathrm{mg}$ melphalan. After four months, a CR was reported, which has been on-going for more than 4 years (53) months.

\section{Discussion}

To our knowledge, we report the largest cohort to date of patients who underwent ILP for Merkel cell carcinoma $(n=10)$. MCC in itself is a rare disease, and locally advanced disease is even rarer. We show that ILP yields a high percentage of complete responses that can be long-lasting in some cases. Loco regional toxicity in our cohort was rather high. However, no firm conclusion can be drawn on both response rates and toxicity due to the small sample size of this study and due to the overall elderly cohort with pre-existing comorbidities.

Literature on ILP in MCC is scarce. A systematic review by Thiels et al. from 2016 found 10 studies that were eligible for inclusion, reporting a total of 16 patients. ${ }^{18}$ High CR rates (86\%) and no major complications were reported. While these rates are very promising and higher than ours (62.5\%), this might be caused by publication bias. Another bias can be assessment of response to ILP, which is performed mostly by physical examination. 
We were unable to analyse response in two patients due to postoperative deaths. No ILP related deaths have been reported in earlier series. Both patients had severe cardiovascular comorbidity, which likely contributed to these postoperative complications. One postoperative death was clearly related to the ILP, with critical ischemia resulting in rhabdomyolysis and progressive renal failure. The extensive atherosclerosis, found on pathological examination of the amputated extremity, showed this patient was at risk for vascular complications. The other postoperative death was an unfortunate case of cardiac decompensation in a patient with extensive cardiovascular disease.

The technique and drugs used in our institutions have been shown to yield low (vascular) complication rates in ILP in melanoma and sarcoma. ${ }^{28-31}$ It is more likely these postoperative deaths were patient-related rather than a reflection of the real risk of postoperative complications after ILP. ${ }^{23}$ Careful patient selection and workup with preoperative vascular studies of the target vessel could identify patients at risk for vascular complications because of peripheral vascular disease, which is more common in the elderly.

Besides ILP, other possible limb-sparing options for loco regionally advanced disease include radiotherapy, laser therapy, isolated limb infusion (ILI), chemotherapy and immunotherapy. ILI is less invasive than ILP, since radiological vascular access is obtained instead of surgical vascular access. Literature on ILI in MCC is as scarce as literature on ILP, with a total of 10 reported patients, some undergoing repeat ILI. ORR ranges between 78 and 100\%, with CR rates between 33 and 75\%. 19,20,32 ILI could be a suitable treatment for older patients with considerable comorbidities that constitute a large part of the MCC population or in case of expected difficult vascular access because of previous lymph node dissection. ${ }^{19}$

Chemotherapy, mostly used in stage IV disease, has shown a response rate of $69 \%$ when used in loco regional disease, however seldom durable. ${ }^{10,11,33,34}$ The potential role of a multimodality approach was illustrated by a series of 46 patients with recurrent MCC. ${ }^{35}$ Median survival was longer for patients treated with a combined modality approach (chemotherapy, radiation, and/or surgery) compared with those treated with a single modality (37 versus 18 months). Perhaps the most promising option for patients with loco regional and/or distant disease is immunotherapy with anti-PD-1 and/or anti-PD-L1. Pembrolizumab has showed promising results in a phase II trial with 26 MCC patients, with an ORR of $56 \%$, with $86 \%$ of responses on- 
going at last follow-up. However, only 2 patients in the trial cohort had loco regionally advanced disease, while the remaining 24 had distant metastases. ${ }^{36}$

Another recent phase II study by Kaufman et al. evaluating avelumab in 88 heavily pre-treated patients with distant metastases, demonstrated a $31.8 \%$ response rate, with a potential of durable responses $(82 \%$ of the responses were on-going at a median follow-up of 10.4 months). ${ }^{37}$ While these studies had a relatively short follow-up time (8 and 10 months), the majority of responses were durable, at least during this interval. The reason for the apparent success of immunotherapy in MCC could be the presence of sufficient neo-antigens through either MCPyV viral antigens, or a high mutational burden through UV damage, allowing the host to mount an effective immune response.

With loco regionally advanced MCC being such a rare disease, the body of evidence supporting specific treatments is small. The current consensus-based guideline does not advocate specific treatment options. ${ }^{38}$ In our cohort, ILP resulted in a high rate of complete responses that were extremely durable in $2 / 8$ cases. We therefore propose ILP as an effective (palliative) option for otherwise uncontrollable loco regional disease, which can even lead to long-term benefit.

\section{Conclusion}

In patients with loco regional MCC, ILP is an effective treatment option providing (possibly durable) local control and limb preservation. Because of the risk of toxicity, careful patient selection and workup is important. 


\section{References}

1. Pectasides D, Pectasides M, Economopoulos T. Merkel cell cancer of the skin. Ann Oncol Off J Eur Soc Med Oncol 2006;17(10):1489-95.

2. Poulsen M. Merkel-cell carcinoma of the skin. Lancet Oncol 2004; 5(10):593-9.

3. Schrama D, Ugurel S, Becker JC. Merkel cell carcinoma: recent insights and new treatment options. Curr Opin Oncol 2012;24(2):141-9.

4. Fields RC, Busam KJ, Chou JF, et al. Five hundred patients with Merkel cell carcinoma evaluated at a single institution. Ann Surg 2011;254(3):465-75.

5. Hui AC, Stillie AL, Seel M, et al. Merkel cell carcinoma: 27-year experience at the Peter MacCallum Cancer Centre. Int J Radiat Oncol Biol Phys 2011;80(5):1430-5.

6. van Veenendaal LM, van Akkooi ACJ, Verhoef C, et al. Merkel cell carcinoma: Clinical outcome and prognostic factors in 351 patients. J Surg Oncol. 2018;117(8):1768-1775.

7. Poulsen M, Round C, Keller J, et al. Factors influencing relapse-free survival in Merkel cell carcinoma of the lower limbea review of 60 cases. Int J Radiat Oncol Biol Phys 2010;76(2):393-7.

8. Harms KL, Healy MA, Nghiem P, et al. Analysis of prognostic factors from 9387 Merkel cell carcinoma cases forms the basis for the new 8th edition AJCC staging system. Ann Surg Oncol 2016;23(11):3564- 71.

9. Grotz TE, Tarantola TI, Otley CC, et al. Natural history of Merkel cell carcinoma following locoregional recurrence. Ann Surg Oncol 2012;19(8):2556-62.

10. Poulsen MG, Rischin D, Porter I, et al. Does chemotherapy improve survival in highrisk stage I and II Merkel cell carcinoma of the skin? Int J Radiat Oncol Biol Phys 2006;64(1):114-9.

11. Tai PTH, Yu E, Winquist E, et al. Chemotherapy in neuroendocrine/ Merkel cell carcinoma of the skin: case series and review of 204 cases. J Clin Oncol 2000;18(12):2493-9.

12. Mojica P, Smith D, Ellenhorn JD. Adjuvant radiation therapy is associated with improved survival in Merkel cell carcinoma of the skin. J Clin Oncol Off J Am Soc Clin Oncol 2007;25(9):1043-7.

13. Strom T, Carr M, Zager JS, et al. Radiation therapy is associated with improved outcomes in Merkel cell carcinoma. Ann Surg Oncol 2016;23(11):3572-8.

14. Ponte P, Vargas-Moniz J, Farricha $\mathrm{V}$, et al. Merkel cell carcinoma: an unusual indication for isolated limb perfusion. Dermatol Online J 2008;14(7). 
15. Moreno-Ramirez D, de la Cruz-Merino L, Ferrandiz L, et al. Isolated limb perfusion for malignant melanoma: systematic review on effectiveness and safety. Oncologist 2010;15(4):416-27.

16. Ross MI. Current status of hyperthermic limb perfusion for in-transit melanoma. Int J Hyperther 2009;24(3):205-17.

17. Gupta A, Heinzman S, Levine EA. Successful treatment of in-transit metastases from Merkel's cell carcinoma with isolated hyperthermic limb perfusion. South Med J 1998;91:289-92.

18. Thiels CA, Gonzalez AB, Gray RJ, et al. Isolated limb perfusion in Merkel cell carcinoma offers high rate of complete response and durable local-regional control: systematic review and institutional experience. J Surg Oncol 2016;114(2):187-92.

19. Zeitouni NC, Giordano CN, Kane 3rd JM, et al. In-transit Merkel cell carcinoma treated with isolated limb perfusion or isolated limb infusion: a case series of 12 patients. Dermatol Surg OffPubl Am Soc Dermatol Surg 2011;37(3):357-64.

20. Turaga KK. Limb preservation with isolated limb infusion for locally advanced nonmelanoma cutaneous and soft-tissue malignant neoplasms. Arch Surg 2011;146(7):870.

21. Olieman AFT, Lienard D, Eggermont AM, et al. Hyperthermic isolated limb perfusion with tumor necrosis factor, interferon gamma, and melphalan for locally advanced nonmelanoma skin tumors of the extremities. Arch Surg 1999;134:303-7.

22. Nieweg OE, Kroon BB. Isolated limb perfusion with melphalan for melanoma. J Surg Oncol 2014;109(4):332-7.

23. Grünhagen DJ, Brunstein F, Graveland WJ, et al. One hundred consecutive isolated limb perfusions with TNF-alpha and melphalan in melanoma patients with multiple in-transit metastases. Ann Surg 2004;240(6):939-47. discussion 47e48.

24. Therasse P, Arbuck SG, Eisenhauer EA, et al. New guidelines to evaluate the response to treatment in solid tumors. European Organization for Research and Treatment of Cancer, National Cancer Institute of the United States, National Cancer Institute of Canada. J Natl Cancer Inst 2000;92(3):205-16.

25. Wieberdink J, Benckhuysen C, Braat RP, et al. Dosimetry in isolation perfusion of the limbs by assessment of perfused tissue volume and grading of toxic tissue reactions. Eur J Cancer Clin Oncol 1982;18(10):905-10.

26. Dindo D, Demartines N, Clavien PA. Classification of surgical complications: a new proposal with evaluation in a cohort of 6336 patients and results of a survey. Ann Surg 2004;240(2):205-13. 
27. Edge SB, Compton CC. The American Joint Committee on Cancer: the 7th edition of the AJCC cancer staging manual and the future of TNM. Ann Surg Oncol 2010;17(6):1471-4.

28. Deroose JP, Eggermont AM, van Geel AN, et al. Long-term results of tumor necrosis factor alpha- and melphalan-based isolated limb perfusion in locally advanced extremity soft tissue sarcomas. J Clin Oncol Off J Am Soc Clin Oncol 2011;29(30):4036-44.

29. Deroose JP, Grünhagen DJ, de Wilt JH, et al. Treatment modifications in tumour necrosis factor-alpha (TNF)-based isolated limb perfusion in patients with advanced extremity soft tissue sarcomas. Eur J Cancer 2015;51(3):367-73.

30. Deroose JP, Grünhagen DJ, van Geel AN, et al. Long-term outcome of isolated limb perfusion with tumour necrosis factor-alpha for patients with melanoma in-transit metastases. Br J Surg 2011;98(11):1573-80.

31. Madu MF, Deken MM, van der Hage JA, et al. Isolated limb perfusion for melanoma is safe and effective in elderly patients. Ann Surg Oncol 2017;24(7):1997- 2005.

32. Steinman J, Ariyan C, Rafferty B, et al. Factors associated with response, survival, and limb salvage in patients undergoing isolated limb infusion. J Surg Oncol 2014;109(5):405-9.

33. Voog E, Biron P, Martin JP, et al. Chemotherapy for patients with locally advanced metastatic Merkel cell carcinoma. Cancer 1999;85: 2589-95.

34. Iyer JG, Blom A, Doumani R, et al. Response rates and durability of chemotherapy among 62 patients with metastatic Merkel cell carcinoma. Cancer Med 2016;5(9):2294-301.

35. Eng TY, Naguib M, Fuller CD, et al. Treatment of recurrent Merkel cell carcinoma: an analysis of 46 cases. Am J Clin Oncol 2004;27(6):576-83.

36. Nghiem PT, Bhatia S, Lipson EJ, et al. PD-1 blockade with pembrolizumab in advanced Merkel-cell carcinoma. N Engl J Med 2016;374(26):2542-52.

37. Kaufman HL, Russell J, Hamid O, et al. Avelumab in patients with chemotherapyrefractory metastatic Merkel cell carcinoma: a multicentre, single-group, open-label, phase 2 trial. Lancet Oncol 2016;17(10):1374-85.

38. Lebbe C, Becker JC, Grob JJ, et al. Diagnosis and treatment of Merkel Cell Carcinoma. European consensus-based interdisciplinary guideline. Eur J Cancer 2015;51(16):2396-403. 



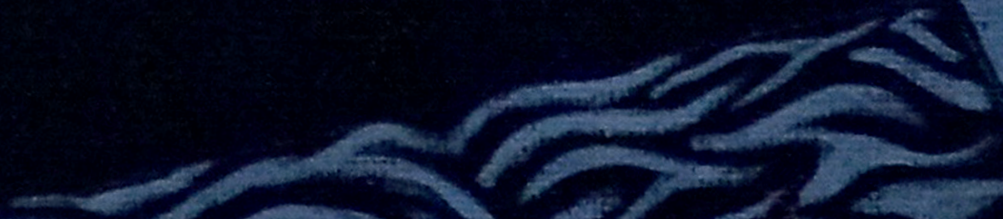

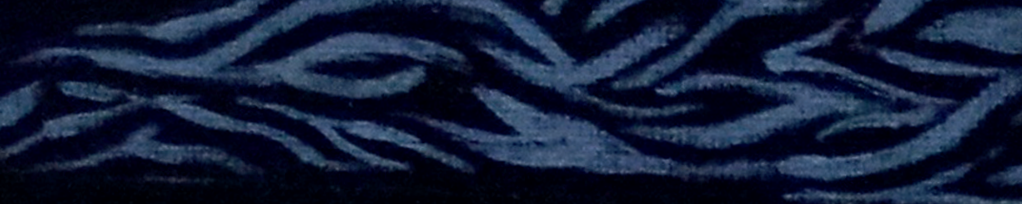




\section{Part III}

Summary, General Discussion and Future Perspectives

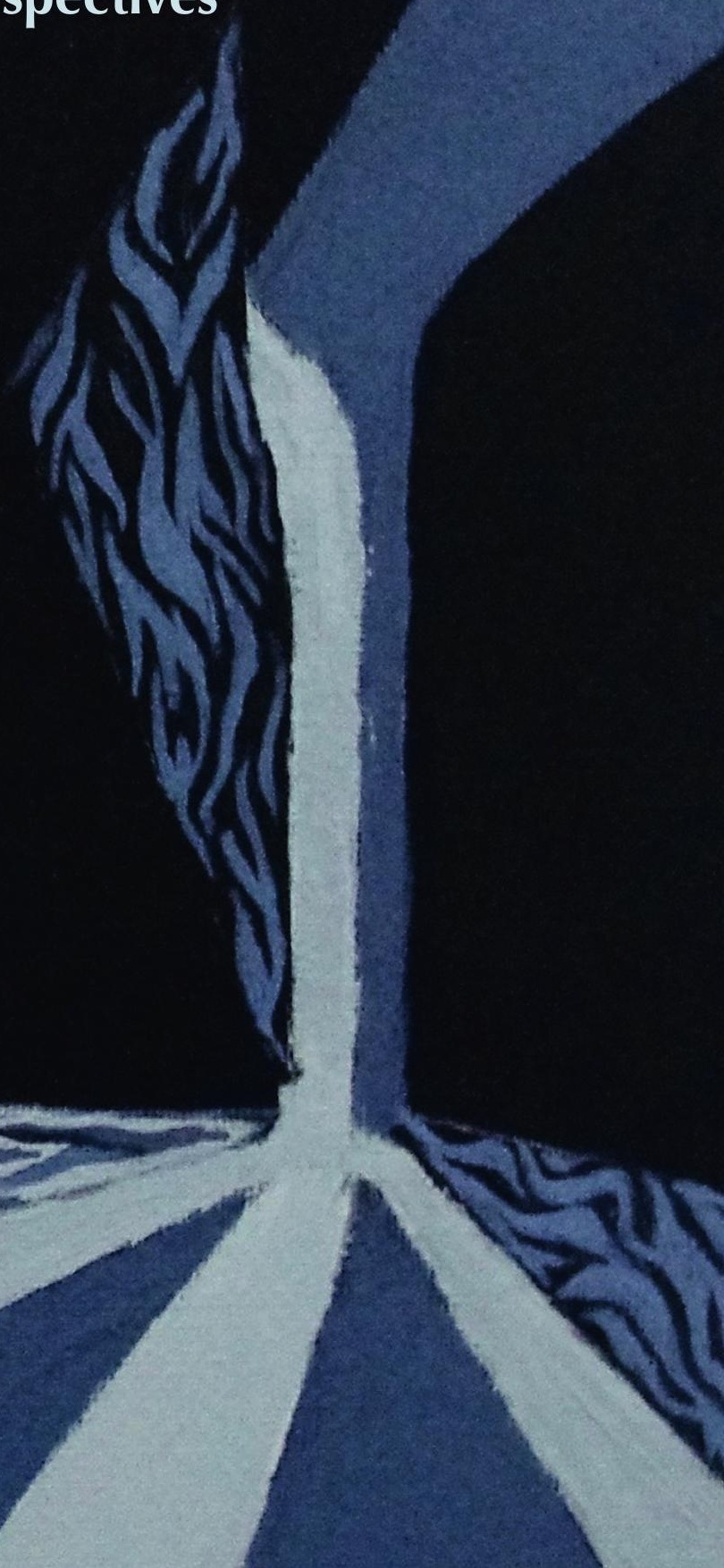




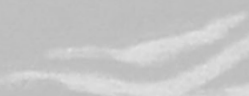

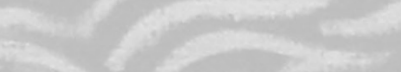

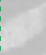

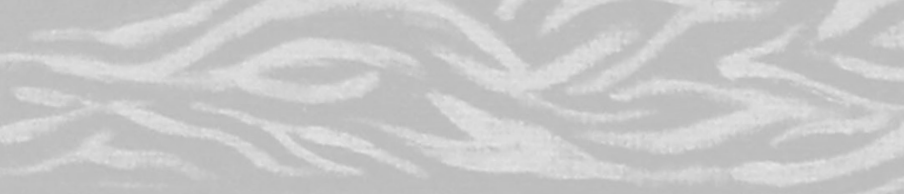


Chapter 9

Summary 



\section{Summary}

The first part of this thesis focuses on prognosis of patients with a neuroendocrine tumour of the small intestine (SI-NET) and Merkel cell carcinoma (MCC), while the second part of this thesis concentrates on various therapies available for patients with these types of NEN.

\section{Part I Prognosis in Neuroendocrine Neoplasia}

In 2010 Modlin et al. developed the first SI-NET nomogram based on clinical and tumour characteristics to estimated survival on patient level, which is evaluated in Chapter 2. It was validated in the largest population to date. In a cohort of 400 patients with a low grade SI-NET the nomogram was able to differentiate between survival for patients in the low-, medium- and high-risk groups $(\mathrm{P}<0.001)$. However, the nomogram underestimated survival for 5 and 10-year disease specific survival (DSS) for most patients in our cohort. This suggests improvement of the nomogram is desirable. Multivariable analysis to evaluate prognostic factors identified age, WHO performance status of 2 , an unknown primary tumour, Ki-67 index $\geq 10 \%$, elevated Chromogranin A $(\mathrm{CgA})>6 \mathrm{x}$ upper limit of normal, and elevated liver tests (any elevation above the upper limit of bilirubin, alkaline phosphatase or glutamyltransferase) as independent predictors for a worse DSS. Ki-67 index $\geq 10 \%$ was the strongest predictor for a worse DSS. These factors could contribute to further improvement of a prognostic model.

In an attempt to identify prognostic factors and new effective targets for precision medicine, the genomic landscape of SI-NETs has been under increasing investigation in recent years. In Chapter 3 we provide an overview of the current literature regarding prognostic and predictive molecular factors in patients with small SI-NETs. This review shows SI-NETs are mutationally silent tumours, whereas epigenetic alterations are more prevalent. An association of (epi)genetic aberrations with prognosis was investigated in 16 of the 35 original studies reviewed, of which 8 found a significant (epi)genetic association for survival or progression. Loss of heterozygosity at chromosome 18, clustered gains of chromosome 4, 5, 7, 14 and $20 p$, copy gain of the SRC gene (a proto-oncogene; involved in cell signalling) and low expression of RASSF1A and P16 were associated with poorer survival. Inactivated tumour suppressor genes, which are more frequently found in SI-NETs, 
are generally unsuitable as targets since restoring the function of tumour suppressor genes is difficult to accomplish. Epigenetic alterations are significantly more common in SI-NETs and may represent more promising targets in the treatment of SI-NETs. Unfortunately at present, predictive or prognostic biomarkers, which can be adopted into clinical practice, have not yet been established.

Literature on MCC is quite limited. Most studies are retrospective and single center. Several large database series have been used to investigate MCC. Unfortunately, these series often lacked data on treatment, recurrence, and cause of death.

Chapter 4 analysed clinical outcome and prognostic predictors in 351 patients with MCC, the second largest institutional cohort to date. Although overall survival (OS) was poor (median 5-year OS 58\%), a relatively low MCC related death was seen (median 5-year MCC related death 22\%). This indicates that a significant number of elderly MCC patients die due to other causes than MCC. In the aged MCC population with high comorbidity, the low MCC related death is important to consider when deciding on a therapy strategy. Post-operative radiotherapy was associated with reduced recurrence of $\mathrm{MCC}(\mathrm{SDH} 0.54 ; \mathrm{Cl} 0.3-0.9)$. The male gender $(\mathrm{SDH} 3.1 ; \mathrm{Cl}$ 1.2-7.9) and loco regional metastasis at time of diagnosis (SDH 2.7; $\mathrm{Cl} 1.1-6.6)$ were negative independent predictors of MCC related death.

To date, no accurate biomarker for patients with MCC has been identified to predict prognosis or response after treatment. The high recurrence rate, the introduction of immunotherapy (IT) and subsequently the improved survival of patients with metastatic MCC emphasize the need for an accurate biomarker guiding treatment decisions. As a prognosticator, serum neuron-specific enolase (NSE) has been validated and is commonly used as a biomarker in gastroenteropancreatic NETs (GEP-NETs) and several other small cell malignancies. Nevertheless, the role of NSE in MCC is still unclear. Chapter 5 shows that NSE corresponds with extent of disease and is able to rule out progression. Extent of disease correlated with NSE $(P=0.01)$. NSE increased with $15.0 \mathrm{ng} / \mathrm{ml}$ with every class e.g. no evidence of disease, (recurrence) primary tumour, in-transit and/or regional metastases or distant metastases. A threshold of $18.2 \mathrm{ng} / \mathrm{ml}$ was considered the most appropriate to rule out progression, reflecting a sensitivity of $91 \%$, specificity of $78 \%$, a positive predicted value of $48 \%$, and negative predicted value of $98 \%$. Furthermore, NSE distinguishes responders from non-responders during IT. This study shows NSE could be a valuable biomarker in patients with MCC. 
In Chapter $\mathbf{6}$ an overview is given of the trials evaluating systemic antitumor treatment in patients with GEP-NETs. The clinical benefit was systematically evaluated with the European Society for Medical Oncology magnitude of clinical benefit scale (ESMO-MCBS) for drugs for solid tumours, and the American Society of Clinical Oncology framework (NHB-ASCO-F) for solid tumours and haematological malignancies. Six out of 35 trials fulfilled all requirements to be assessed with the ESMO-MCBS resulting in an ESMO-MCBS score of 2 or 3, while only 9 trials could be assessed with the NHB-ASCO-F and resulted in scores between 37.6 and 57.4. The currently used systemic treatments for patients with GEP-NETs had low scores according to the NHB-ASCO-F, and none could be graded as meaningful clinical beneficial according to the ESMO-MCBS. The highest scores included the RADIANT3 trial, which analysed everolimus versus placebo in patients with advanced progressive NET and the NETTER-1 trial, which investigated 177Lu-dotatate with octreotide LAR versus octreotide LAR alone in patients with progressive metastatic midgut NET.

The presence of liver metastases (LM) is a strong negative prognostic factor and causes significant morbidity for NET patients. Therefore, treatment strategies to obtain control of LM are not only relevant in terms of prognosis but also to achieve symptom control of functional tumours, which could increase quality of life.

Chapter 7 is the first comparative study of trans arterial embolization (TAE) and selective internal radiation therapy (SIRT) that evaluated toxicity, response and survival. Furthermore, a subgroup analysis was performed for SI-NET and pancreatic NET, the most prevalent locations of a primary NET. It shows TAE and SIRT are safe and effective treatment modalities for patients with liver dominant metastatic disease. Nevertheless, they show different toxicity and response profiles. A total of 86 patients underwent TAE or SIRT, of whom 54 patients (63\%) underwent TAE and 32 patients $(37 \%)$ underwent SIRT. In total, 130 procedures were performed. Overall, the total response rates did not differ ( $\mathrm{P}=0.61 ; 80 \%$ and $84 \%$ for TAE and SIRT, respectively), but functional symptoms might decrease more often after TAE compared to SIRT $(\mathrm{P}=$ $0.002 ; 82 \%$ and $42 \%$, respectively), especially in SI-NETs. No differences in survival rates were observed, but a trend was seen towards better survival after SIRT in patients with LM of a pancreatic NET ( $\mathrm{P}=0.053$; median 7 months and 38 months for TAE and SIRT, respectively). 
No standard treatment for loco regionally advanced MCC is yet established. In Chapter 8, the largest cohort to date of patients with MCC who underwent isolated limb perfusion (ILP) was presented. A total of ten patients with loco regional MCC underwent ILP. No signs of systemic toxicity were present post-ILP. Severe loco regional toxicity (compartment syndrome) occurred in one patient and another elderly patient with extensive atherosclerosis had to undergo trans femoral amputation due to critical ischemia. Eight patients could be included for response evaluation and survival analysis. The overall response rate was $87.5 \%$ with a complete response rate of $62.5 \%$. Two long-term responses of 53 months and 71 months were observed. Median loco regional progression free survival was 5 months and median OS was 54 months. Our study shows that ILP is an effective treatment option providing (possibly durable) local control and limb preservation. Because of the risk of toxicity, careful patient selection and workup is important. 



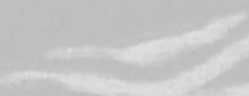

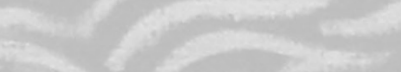

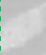

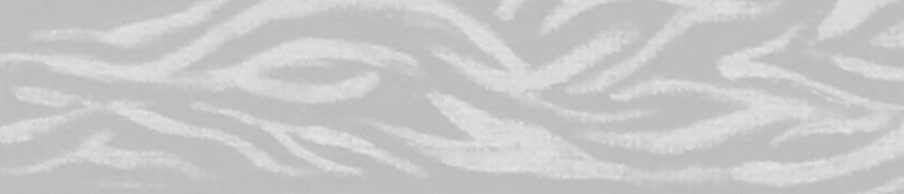




\section{Chapter 10}

General discussion and future perspectives 



\section{General discussion}

The Netherlands Cancer Institute (NCI) and UMC Utrecht (UMCU) form an European Neuroendocrine Tumour Society (ENETS) Centre of Excellence (CoE) specialized in treating patients with neuroendocrine neoplasia (NEN). Because of this collaboration patients can benefit from an experienced multidisciplinary team (MDT) providing them with all the current relevant treatment options. Furthermore, it provides research opportunities due to concentrated care in a relatively large patient population with detailed information on treatment and survival. Moreover, in the field of oncology we have shifted to a more personalized cancer care. ${ }^{1}$ Essential for personalized cancer care is insight on prognosis and treatment with respect to the various tumour and patient characteristics. Using the experience of the MDT and the collected data on outcomes of the patients treated in this ENETS CoE, this thesis provides insight in prognostic predictors and effects of various treatment modalities in patients with a neuroendocrine tumour of the small intestine (SI-NET) and patients with Merkel cell carcinoma (MCC), respecting the heterogeneity of NEN. By doing so, a beginning was made for a more tumour specific and patient tailored approach of cancer care in NEN.

- Neuroendocrine tumour of the small intestine

Despite the identification of several prognostic factors, accurate prognostication in individual patients with a SI-NET remains challenging due to their heterogeneous disease course. ${ }^{2-4}$ Several studies have identified prognostic factors mainly based on clinical and tumour characteristics. Also, in recent years the genomic landscape of SI-NETs has been under increasing investigation and identified several molecular prognostic factors. Nevertheless, their role in daily clinical practice remained limited. As a consequence, there remains an unmet need for adequate prognostication to predict disease course and survival for individual patients with SI-NETs.

A nomogram based on clinical data and tumour characteristics designed by Modlin et al. was the first extensive attempt for individual prognostication. Validation is vital before it can be used in daily clinical practice. ${ }^{5}$ The nomogram was validated in the largest population to date of patients with a SI-NET $(n=400) .{ }^{6}$ Although it was based on a large dataset from 7.455 patients from the Surveillance, Epidemiology and End Results (SEER) registry and variables were selected and weighed after extensive analyses of literature-curated data, the nomogram itself was initially validated in only 
33 patients. Two other studies have attempted to validate the predictive properties of the SI-NET nomogram. ${ }^{7,8}$ However, these studies were performed in either a small cohort of patients $(n=70)$ included during an unspecified study period, while the other concerned patients $(n=121)$ that underwent surgery with curative intent underwent (and therefore differed in a-priori survival probability). In a large and representative cohort of patients with SI-NETs from our NCI-UMCU ENETS CoE, the nomogram showed its potential for prognostication. The nomogram was able to differentiate between survival for patients in the low-, medium- and high-risk groups. However, it underestimated survival for most patients.

The underestimation of survival could be explained by the fact that the nomogram was based on studies from 1997 and 2010 and on SEER data from patients diagnosed between 1977-2007. Several studies have investigated survival of patients with NET over different time periods and found that survival increases in patients diagnosed in more recent time periods..$^{9,10}$ This might be due to emerged systemic treatment modalities, which have a beneficial effect on NET-related survival. These therapies include mainly somatostatin receptor analogues (SSA) and more recently peptide receptor radionuclide therapy (PRRT). SSA made an entrance in 1987 and was the first systemic treatment option specifically for NET. Initially, SSA's were found to reduce symptoms of carcinoid syndrome, but an antiproliferative effect was shown in the PROMID and CLARINET study. ${ }^{11-12}$ PRRT was introduced in 2008. The development of PRRT is generally considered one of the biggest enhancements in recent years. This treatment uses a radiolabeled somatostatin analogue to achieve local intratumoral nuclear therapy and showed a survival benefit for patients with GEP-NET treated with PRRT in the NETTER-1 study. ${ }^{13}$ Nonetheless, PRRT was only limited available during their and our study period. Moreover, diagnostic techniques for NETs have improved, it is likely earlier detection contributes to an improved survival. ${ }^{14}$ As a consequence, the nomogram was based on a poorer survival outcome compared to our cohort. This findings suggests the nomogram in its current form, unfortunately, seems insufficient to accurately predict individual prognosis.

In our large and representative cohort of patients with a SI-NET possible prognosticators were investigated. These factors could contribute to further development of a prognostic model, such as a nomogram. Age, a WHO performance status of 2, an unknown primary tumour, Ki-67 index $\geq 10 \%$, elevated Chromogranin A $(\mathrm{CgA})>6 \mathrm{x}$ upper limit of normal (ULN), and elevated liver tests were identified as independent predictors for a worse disease specific survival (DSS). 
An elevated CgA of $>6 x$ ULN was associated with a shorter DSS. CgA is a product of the neuroendocrine cell secretory granule. It has been variably reported to correlate with tumour biology and mass and prognosticate survival. ${ }^{15,16}$ Several studies have shown the prognostic value of baseline $\mathrm{CgA}$, while others have discussed that a change of CgA (of 25-20\%) might be a better prognostic predictor than a single measurement. ${ }^{17}$ However this needs further validation.

Remarkably, liver metastases did not prove to be an independent prognostic predictor for DSS while many other studies did show this association. ${ }^{18-21}$ Yet, any elevation of liver tests was associated with a worse DSS. Although the presence of liver metastases alone was not identified as an independent prognostic predictor, it supports the notion that the extent of liver metastases or liver tumour load (resulting in elevated liver tests), is more important than the presence of liver metastases alone. $^{22}$

A Ki-67 index $\geq 10 \%$ was identified as the strongest predictor for a worse DSS. This cut-off value is currently not used in clinical practice. ${ }^{23}$ The cut-off point most suited for distinguishing between a grade I and II NET has been the subject of debate since the introduction of the Ki-67 index in the ENETS grading system in $2007 .{ }^{24}$ In a systematic review by Richards-Taylor et al. it was postulated that grade II and Ki-67 index $\leq 5 \%$ NET was more similar to grade I NET, compared to a grade II NET with a $\mathrm{Ki}-67$ index $>5-20 \%{ }^{25}$ Although in our cohort, a Ki-67 index $<5 \%$, or Ki-67 index $<10 \%$ could not be identified as a separate prognostic factor for DSS, it does suggests that the current Ki-67 index subdivision used for grading NETs could be improved for a better prognostication. Future studies should aim to determine which cut-off value, if any, albeit in combination with other histopathological characteristics, would be most suitable.

In recent years, basic research in SI-NETs has attempted to unravel the molecular events underlying NET tumorigenesis. ${ }^{26}$ This thesis provided an overview of the recent studies evaluating molecular characteristics of SI-NETs with a focus on their predictive and prognostic significance.

A total of 35 original studies were reviewed. Unfortunately, in only 16 of 35 studies an association with prognosis was investigated, of which eight found a significant (epi)genetic association for survival or progression. Nevertheless, predictive or prognostic molecular biomarkers have not yet been established. SI-NETs are mutationally silent tumours however molecular subgroups have been identified, one subgroup which is characterised by loss of heterozygosity $(\mathrm{LOH})$ of chromosome 18 
as an early event and the other subgroup which has no alterations of chromosome 18 but shows clustered gains on chromosomes $4,5,7,14$ and $20 .{ }^{27-31}$

$\mathrm{LOH}$ at chromosome 18 remains the most frequent genomic aberration (44-100\%) found in SI-NETs. The clinical significance of LOH of chromosome 18 has been evaluated in multiple studies, either focussing solely on LOH of chromosome 18 or as part of a molecular profile study with variable effects on survival. ${ }^{32}$ Likewise, a previously identified association of gain of chromosome 14 leading to a higher tumour grade and a worse survival could not be corroborated. ${ }^{31}$ Subsequently, its exact consequences for survival need further evaluation. Furthermore, copy gain of the SRC gene (proto-oncogene; involved in cell signalling), which was present in $25 \%$ of SI-NETs, was associated with poorer prognosis $(P=0.047) .{ }^{33}$ The SRC gene was also the most commonly amplified oncogene (23\%) in the study by Banck et al and could potentially be a novel prognostic biomarker. ${ }^{34}$

Nonetheless, the rarity of SI-NETs has hampered conducting sizeable clinical trials involving large-scale integrated genomic analysis. Moreover, real patient data is vital to prognostically interpret obtained genomic data. Only 3 studies investigating a prognostic association, had a patient population $>100$ patients. ${ }^{35-37}$ Validation of these results in an independent and larger cohort is required before translation into clinical practice is possible.

Besides improving prognostication, genetic information could be used to guide treatment decisions. Generally, inactivated tumour suppressor genes, which are found in SI-NETs, are unsuitable as targets since restoring the function of tumour suppressor genes is difficult to accomplish. Mutations in oncogenes, which should be easier to target, have only recently been described in small numbers in SI-NETs and thus far no clinical studies have been undertaken to target these mutations in SINETs. Genetic alterations in the P13K/AKT/mTOR were found in primary SI-NETs and liver metastasis, providing a rationale for the use of mTOR inhibitors. ${ }^{34}$ However, a correlation between efficacy and mTOR mutational status prior to commencement of therapy with mTOR inhibitors has not yet been investigated.

Nevertheless, the expanding molecular knowledge on SI-NETs is encouraging. In comparison with genetic mutations, epigenetic alterations are significantly more common in SI-NETs. ${ }^{38}$ Epigenetic changes include DNA methylation, histone modifications and the actions of miRNA. Our search yielded studies that showed DNA methylation changes in $65-82 \%$ of patients with SI-NETs and multiple miRNA 
deregulations. ${ }^{39,40}$ Specific genes such as RASSF1A, SEMA3F and CTNNB1 are hypermethylated in SI-NETs silencing their transcription and a low expression of RASSF1A was associated with poorer survival. ${ }^{41}$ These processes can be pharmacologically modified by targeting enzymes involved in DNA methylation and histone modifications, and by miRNA inhibitors thereby representing an appealing target for therapy and may represent promising targets in the treatment of SI-NETs. ${ }^{42-}$ 43

This thesis also focussed on currently available therapies for patients with neuroendocrine tumours (NETs). First, the clinical benefit of systemic treatments in gastroenteropancreatic NETs (GEP-NETs) was assessed. Recently, several tools have been developed to grade the clinical benefit of cancer drugs. Systemic treatment in patients with GEP-NETs was systematically evaluated with the European Society for Medical Oncology magnitude of clinical benefit scale (ESMO-MCBS) for drugs for solid tumours, and the American Society of Clinical Oncology framework (NHBASCO-F) for solid tumours and haematological malignancies. ${ }^{44,45}$ The currently used systemic treatments for patients with GEP-NETs had low scores according to the NHB-ASCO-F, and none could be graded as meaningful clinical beneficial according to the ESMO-MCBS. The scores were generally lower than in other tumour types, like metastasized breast or colorectal cancer. ${ }^{46,47}$ Ideally, OS or DSS should be the primary end points of trials evaluating new drugs. ${ }^{48} \mathrm{OS}$ and DSS difference detection is challenging in patients with SI-NETs due to the long natural course of the disease and the wide variability of subsequent lines of therapy after progression.

Furthermore, in more common tumour types, sufficient numbers of patients can be included in trials powered to detect a difference in survival between the intervention and control group. Therefore, in GEP-NETs such large trials are scarce.

Another important factor to take into consideration when evaluating the clinical benefit of systemic treatment is the heterogeneous patient population. The eligibility criteria of the RADIANT-2 trial included patients with serotonin producing NET and

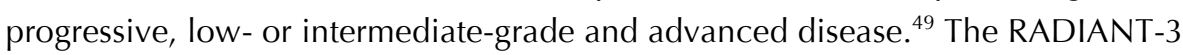
and RADIANT-4 trial included pancreatic NETs and non-functional advanced NET, The NETTER-1 included metastatic midgut NET, while the CLARINET trial included GEP-NET or patients with NET of unknown origin with a Ki-67 $<10 \% .{ }^{12,13,50,51}$ Notwithstanding these clear criteria, large variation in patient and tumour characteristics between patients still existed. Despite the randomized design of the trial, this could have influenced PFS. This stresses the need for either 
subgroup analyses or better pre-defined patient populations to be able to aid personalized treatment decisions.

Besides systemic treatment, another frequently used treatment modality for patients with a NET is trans-arterial liver directed therapy (LDT). ${ }^{52,53}$ Approximately, nearly half of the patients have metastases at time of diagnosis, predominantly in the liver and during the course of their disease, up to an estimated $90 \%$ of the patients will ultimately develop liver metastases (LM) affecting survival. ${ }^{54-57}$ In case of LM, serotonin and other vasoactive mediators can not be (completely) metabolized by the liver, inducing functional symptoms e.g. carcinoid syndrome. ${ }^{58}$ Therefore, treatment strategies to obtain control of LM are not only relevant in terms of prognosis but also to achieve symptom control, which could increase quality of life. Trans arterial embolization (TAE) and selective internal radiation therapy (SIRT) are frequently used LDT for patients with liver dominant metastatic disease. ${ }^{59,60}$

To our knowledge, this thesis presents the first head-to-head comparison of TAE and SIRT including toxicity, response and survival analyses. Previously, Elf et al. investigated response and toxicity after TAE and SIRT in 11 patients with a SI-NET. ${ }^{61}$ An equal biochemical response and radiological response was shown in patients with metastatic NET. However, the small sample size is a major limitation of this study. Chen et al. retrospectively evaluated a cohort of 155 patients. ${ }^{62}$ They identified prognostic factors and analysed survival in patients with a NET undergoing embolotherapies. Tumour grade was identified as a prognostic factor. Yet, tumour grade was unequally distributed between the treatment groups. Likewise, the primary site of origin was recognized as a prognostic factor, but no subgroup analyses were performed. Another important limitation was the absence of a response evaluation. Therefore, the results of this study are susceptible to confounding.

We showed both TAE and SIRT are safe and effective treatment modalities. The total response rates did not differ, but functional symptoms in SI-NET seemed to decrease more often after TAE compared to SIRT $(P=0.02)$ when performing subgroup analysis. No differences in survival rates were observed. Nevertheless, TAE and SIRT showed different toxicity profiles. The different toxicity and response profiles indicates individual patient characteristics should be taken into account when deciding on a treatment regime, such as the origin of the NET, presence of functional symptoms or the different toxicity profiles in the context of the patient's comorbidities. 
- Merkel cell carcinoma

In this thesis, to our knowledge, the second largest population of patients with MCC was presented. It confirmed the poor OS and high relapse rate reported in other studies. However we found a lower 5-year MCC related death (22\%). This indicates that a significant number of elderly MCC patients die due to other causes than MCC. In the aged MCC population with high comorbidity, the low MCC related death is important to consider when deciding on a potential aggressive treatment strategy.

The male gender was our strongest prognostic factor (SDH 3.1) associated with a higher MCC related death rate. Others have seen this relation as well but the exact mechanism remains unknown. ${ }^{63-67}$ Sridharan et al. linked this association to a higher nodal involvement, which also proved a prognostic factor for a higher MCC related death (SDH 2.7). While Jabbour et al. sought the explanation for the disparity in overall survival in different patient behaviour (e.g. smoking) and delayed presentation, as is often presumed in patients with melanoma. ${ }^{68}$ However, these explanations seems unlikely since we analysed MCC related death and corrected for size and nodal stage, which would reflect a delayed presentation.

Gender related disparities concerning the immune system are already known, for instance in the prevalence of autoimmune disease. ${ }^{69}$ Furthermore, there is significant evidence that the immune system plays a role in developing MCC and could possibly affects survival. MCC is associated with the Merkel cell Polyomavirus (MCPyV), the use of immunosuppressive medication and the immune system is suggested to be responsible for the spontaneous regression of the primary tumour. ${ }^{70-72}$ Hence, we believe the difference in survival between genders could be immune related. More research in needed to clarify the exact mechanism responsible for the worse survival of the male patients.

Treatment of MCC consists mainly of surgical treatment followed by post-operative radiotherapy (RT) for early stage MCC. ${ }^{73}$ In our cohort, we confirmed the association of post-operative radiation therapy (RT) with reduced recurrence (SDH 0.54). No standard treatment for loco regionally advanced disease is yet esthablished. ${ }^{74}$ In patients with loco regionally advanced melanoma, isolated limb perfusion (ILP) of the extremities proved to be safe and effective. ${ }^{75}$ This thesis reports the largest cohort to date of patients who underwent ILP for Merkel cell carcinoma $(n=10)$ bearing in mind MCC in itself is a rare disease, and locally advanced disease is even rarer. In our cohort, ILP yields a high percentage of complete responses (5/8) that can be long- 
lasting in some cases. Due to the small sample size, no significant difference in survival between responders could be found. Because of the risk of toxicity, careful patient selection and workup is important. Especially, considering the recent findings in immunology and results from treating patients with immune checkpoint inhibitors (anti-PD-1 and/or anti-PD-L1).

These immune checkpoint inhibitors have completely changed the treatment landscape and outlook for metastatic MCC patients. ${ }^{76-78}$ Pembrolizumab has showed promising results in a phase II trial with 26 MCC patients, with an ORR of $56 \%$, with $86 \%$ of responses on-going at last follow-up. Another recent phase II study by Kaufman et al. evaluating avelumab in 88 heavily pre-treated patients with distant metastases, demonstrated a $31.8 \%$ response rate, with a potential of durable responses ( $82 \%$ of the responses were on-going at a median follow-up of 10.4 months). The introduction of immunotherapy (IT) and subsequently the improved survival of patients with metastatic MCC emphasize the need for an accurate biomarker guiding treatment decisions. ${ }^{79}$ To date, no accurate serum biomarker for patients with MCC has been identified to predict prognosis or response after treatment. ${ }^{80}$

The use of serum NSE as a biomarker is already validated as a prognosticator in small cell carcinomas such as small cell lung carcinoma and neuroblastoma. Several studies have showed its relevance in evaluating responsiveness to therapy and detecting early recurrences for these malignancies. ${ }^{81-83}$ Nevertheless there is scarce data about the correlation of serum NSE and MCC. In a large study evaluating NSE in patients with MCC, we showed a significant correlation between NSE and extent of disease $(P=0.01)$. The outstanding performance of NSE in detecting progression was expressed by an AUC of 0.89. Several threshold values were analysed. For clinical use, we considered a negative redicted value (NPV) the most relevant parameter, bearing in mind the potential aggressive behaviour of MCC. The threshold of 18.2 $\mathrm{ng} / \mathrm{ml}$ calculated with the Youdens' index resulted in a NPV of $98 \%$, comparable to the NPV of lower threshold values but with considerable less false positive evaluations. In patients receiving IT $(n=23)$, NSE was able to distinguish responders from non-responders.

Furthermore, NSE and its association with recurrence and OS was evaluated. Both baseline and postoperative NSE (continuous and using a cut-off of $18.2 \mathrm{ng} / \mathrm{ml}$ ) were not associated with recurrence or OS. The absent prognostic association of NSE 
could be explained by the little number of baseline samples. Availability of more baseline samples might be needed to establish an association. Nevertheless, it is possible NSE serum level is primarily a reflection of tumour burden. Thus a prognostic association might be lacking, even when more baseline samples could be obtained.

Besides NSE, antibodies against MCPyV-encoded oncoproteins could be a potential promising biomarker showing comparable results to NSE in detecting progression but also show a prognostic association. ${ }^{84}$ However depending on the population, approximately up to $50 \%$ of patients have a non-MCPyV associated MCC and would not produce antibodies against MCPyV-encoded oncoproteins, hampering the use of these antibodies as a biomarker. ${ }^{85}$ NSE is not impeded by the MCC subtype, either MCPyV- or UV associated subtype. Despite the lacking prognostic value, NSE could prove to be a valuable biomarker for patients with MCC. 



\section{Future perspectives}

Prognostication and personalized cancer treatment is a continuously evolving and improving process. New statistical approaches, diagnostic techniques and the development of new treatments or treatment strategies challenge long-standing results and just recent findings, aiming to optimize cancer care for patients. Likewise, the findings in this thesis should be reflected within other recent developments research in neuroendocrine neoplasia (NEN), identifying the necessary steps for further advancement of an optimal patient tailored prognosis and treatment approach.

A nomogram is often used to model prognostic factors in order to estimate individual prognosis. ${ }^{5}$ The nomogram for patients with a SI-NET in its current form, unfortunately, seems insufficient to accurately predict individual prognosis. In the last decades, the genomic landscape of SI-NETs has been under increasing investigation and identified several molecular prognostic factors. More recently, the development of biomarkers such as a blood-derived multianalyte assay (e.g. the NETest), in which circulating transcript analysis can provide an individual tumour profile, showed promising results for diagnosing and monitoring disease. ${ }^{86-89}$ The prognostic ability of the NETest is already suggested and validation of the NETest is underway to evaluate its prognostic value. ${ }^{86,88}$ Furthermore, in this thesis, several prognostic factors (Ki-67 index, Chromogranin A, elevated liver test as a reflection liver tumour burden) were suggested to improve an individualized estimation of prognosis. A model taking into account both molecular, clinical and patient characteristics might be best suited for prognostication. In the coming years, large multi-institutional cohorts with the advantage of complete datasets and adequate patient populations are necessary to allow extensive prognostic modelling and external validation which correlate (molecular) prognostic factors and to real clinical outcomes.

Furthermore, (epi)genetic research should aim to identify targetable (epi)genetic alterations. An increasing number of drugs targeting DNA methylation and histone methylation have been developed and successfully tested pre-clinically which are currently in evaluation in phase I-III clinical trials. ${ }^{90}$ Additionally, the more specific upregulation of miRNAs in SI-NETS may provide actionable targets since multiple strategies for miRNA-based therapies are under investigation. ${ }^{91}$ Larger studies combined with evolving molecular technologies might lead to a more effective treatment strategy in which patients with specific molecular tumour profiles will be 
selected for targeted pharmaceutical interventions. In addition, the recent development of stem cell derived organoids from patients with SI-NETs could be very promising. ${ }^{92}$ Organoids harbour patient-specific genetic and epigenetic information and can be used for drug screening. In that way, organoids could be used to understand tumour biology and to develop and asses response to treatment.

Compared to SI-NETs, molecular characterization in MCC remains limited. The panNENomics initiative aims to develop a bio bank for (among others) MCC to elucidate molecular pathways leading to the development this malignacy. ${ }^{93}$ Previously performed molecular studies have focussed on identifying the 2 subtypes of MCC, an UV radiation/high mutational burden associated subtype and a subtype associated with the Merkel cell Polyomavirus and a low mutational burden with both favourable responses on IT. ${ }^{94}$ In addition, it showed that IT administered as a first-line therapy result in a higher response rate. At present trials are in progress investigating IT in a (neo)adjuvant setting. ${ }^{95}$ Future (molecular profiling) studies could aim to identify which patients benefit most from IT. Bearing in mind the potential expanding indications for IT, it is desirable that NSE will be evaluated in these clinical settings as well.

Unfortunately unlike in MCC and other malignancies, the role of IT in SI-NETs is yet to be defined. In the KEYNOTE-28 trial pembroluzimab showed only limited antitumor activity. ${ }^{96}$ Although, these results could be expected considering the low mutational burden of SI-NETs, in some patients durable responses were observed. Using PRRT as a releaser of neoantigens in combination with IT, intending to enhance the effect of IT in SI-NETs, has been proposed. ${ }^{97}$ Future trails could explore expanding indications for PRRT in combination with IT, introducing one of the most promising therapies in MCC for SI-NETs as well.

Concluding, several options for optimization of prognostic factors and therapeutic strategies remain. Regardless which direction future research will take, patient care and research in NEN, either SI-NETs or MCC, benefits from a multidisciplinary approach in an experienced multidisciplinary team with accessibility to all the current relevant treatment options to ensure an individualized and a high level of care with adequate implementation of recent developments. 


\section{References}

1. Bode AM, Dong Z. Precision oncology- the future of personalized cancer medicine? NPJ Precis Oncol. 2017 Mar 20;1(1):2.

2. Modlin IM, Oberg K, Chung DC, et al. Gastroenteropancreatic neuroendocrine tumours. Lancet Oncol. 2008 Jan;9(1):61-72.

3. Cives M, Soares HP, Strosberg J. Will clinical heterogeneity of neuroendocrine tumors impact their management in the future? Lessons from recent trials. Curr Opin Oncol. 2016 Jul;28(4):359-66

4. Oronsky B, Ma PC, Morgensztern D, et al. Nothing but NET: a review of neuroendocrine tumors and carcinomas. Neoplasia. 2017;19(12):991-1002.

5. Balachandran VP, Gonen M, Smith JJ, et al. Nomograms in oncology: more than meets the eye. Lancet Oncol. 2015;16(4):e173-80.

6. Modlin IM, Gustafsson BI, Pavel M,et al. A nomogram to assess small-intestinal neuroendocrine tumor ('carcinoid') survival. Neuroendocrinology 2010;92:143-57.

7. Clift AK, Faiz O, Goldin R, et al. Predicting the survival of patients with small bowel neuroendocrine tumours: comparison of 3 systems. Endocrine connections 2017;6:71-81.

8. Kelly S, Aalberg J, Agathis A, et al. Predicting Survival of Small Intestine Neuroendocrine Tumors: Experience From a Major Referral Center. Pancreas 2019;48:514-8.

9. Yao JC, Hassan M, Phan A, et al. One hundred years after "carcinoid": epidemiology of and prognostic factors for neuroendocrine tumors in 35,825 cases in the United States. Journal of clinical oncology : official journal of the American Society of Clinical Oncology 2008;26:3063-72.

10. Dasari A, Shen C, Halperin D, et al. Trends in the Incidence, Prevalence, and Survival Outcomes in Patients With Neuroendocrine Tumors in the United States. JAMA oncology 2017;3:1335.

11. Rinke A, Wittenberg M, Schade-Brittinger C, et al. Placebo Controlled, Double Blind, Prospective, Randomized Study on the Effect of Octreotide LAR in the Control of Tumor Growth in Patients with Metastatic Neuroendocrine Midgut Tumors (PROMID): Results on Long Term Survival. Neuroendocrinology 2017;104(1):26-32.

12. Caplin ME, Pavel M, Ćwikła JB, et al. CLARINET Investigators. Lanreotide in metastatic enteropancreatic neuroendocrine tumors. N Engl J Med. 2014 Jul 17;371(3):224-33. 2018;100:23-9.

13. Strosberg JR, Wolin EE, Chasen BA, et al. First update on overall survival, progressionfree survival, and health-related time-to-deterioration quality of life from the NETTER- 
1 study: $177 \mathrm{Lu}-$ Dotatate vs. high dose octreotide in progressive midgut neuroendocrine tumors. JCO. 2018;36(15 supplemental):4099.

14. Hallet J, Law CH, Cukier M, Saskin R, Liu N, Singh S. Exploring the rising incidence of neuroendocrine tumors: a population-based analysis of epidemiology, metastatic presentation, and outcomes. Cancer. 2015;121(4):589-97.

15. Chou WC, Chen JS, Hung YS, et al. Plasma Chromogranin A Levels Predict Survival and Tumor Response in Patients with Advanced Gastroenteropancreatic Neuroendocrine Tumors. Anticancer research 2014;34:5661-70.

16. Jensen $\mathrm{KH}$, Hilsted $\mathrm{L}$, Jensen $\mathrm{C}$, et al. Chromogranin $\mathrm{A}$ is a sensitive marker of progression or regression in ileo-cecal neuroendocrine tumors. Scandinavian journal of gastroenterology 2013;48:70-7.

17. Rossi RE, Ciafardini C, Sciola V, Conte D, Massironi S. Chromogranin A in the follow-up of gastroenteropancreatic neuroen- docrine neoplasms: is it really game over? A systematic review and meta-analysis. Pancreas. 2018;47(10):1249-1255.

18. Lesen E, Granfeldt D, Berthon A, et al. Treatment Patterns and Survival among Patients with Metastatic Gastroenteropancreatic Neuroendocrine Tumours in Sweden - a Population-based Register-linkage and Medical Chart Review Study. Journal of Cancer 2019;10:6876-87.

19. Pape UF, Berndt U, Muller-Nordhorn J, et al. Prognostic factors of long-term outcome in gastroenteropancreatic neuroendocrine tumours. Endocrine-related cancer 2008;15:1083-97.

20. Carmona-Bayonas A. Prediction of Progression-Free Survival in Patients With Advanced, Well-Differentiated, Neuroendocrine Tumors Being Treated With a Somatostatin Analog: The GETNE-TRASGU Study. Journal of Clinical Oncology $2019 ; 37$.

21. Laskaratos FM, Walker M, Wilkins D, et al. Evaluation of clinical prognostic factors and further delineation of the effect of mesenteric fibrosis on survival in advanced midgut neuroendocrine tumours. Neuroendocrinology 2018.

22. Laskaratos FM, Walker M, Naik K, et al. Predictive factors of antiproliferative activity of octreotide LAR as first-line therapy for advanced neuroendocrine tumours. British journal of cancer 2016;115:1321-7.

23. Rindi G, Klimstra DS, Abedi-Ardekani B, et al. A common classification framework for neuroendocrine neoplasms: an International Agency for Research on Cancer (IARC) and World Health Organization (WHO) expert consensus proposal. Mod Pathol. 2018;31(12):1770-1786. 
24. Khan MS, Luong TV, Watkins J, et al. A comparison of Ki-67 and mitotic count as prognostic markers for metastatic pancreatic and midgut neuroendocrine neoplasms. British journal of cancer. 2013;108(9):1838-45.

25. Richards-Taylor S, Ewings SM, Jaynes E, et al. The assessment of Ki-67 as a prognostic marker in neuroendocrine tumours: a systematic review and meta-analysis. Journal of clinical pathology 2016;69:612-8.

26. Stålberg P, Westin G, Thirlwell C. Genetics and epigenetics in small intestinal neuroendocrine tumours. J Intern Med. 2016 Dec;280(6):584-594.

27. Hashemi J, Fotouhi O, Sulaiman L, et al. Copy number alterations in small intestinal neuroendocrine tumors determined by array comparative genomic hybridization. BMC Cancer 2013;13:505.

28. Kulke MH, Freed E, Chiang DY, et al. High-resolution analysis of genetic alterations in small bowel carcinoid tumor reveals areas of recurrent amplification and loss. Genes, Chromosomes and Cancer 2008;47:591-603.

29. Andersson E, Swärd C, Stenman G, et al. Highresolution genomic profiling reveals gain of chromosome 14 as a predictor of poor outcome in ileal carcinoids. EndocrineRelated Cancer 2009;16:953-966.

30. Karpathakis A, Dibra H, Pipinikas C, et al. Prognostic impact of novel molecular subtypes of small intestinal neuroendocrine tumor. Clin Cancer Res. 2016;22(1):250258.

31. Cunningham JL, Diaz de Ståhl T, Sjöblom T, et al. Common pathogenetic mechanism involving human chromosome 18 in familial and sporadic ileal carcinoid tumors. Genes Chromosomes Cancer. 2011;50(2):82-94.

32. Kim DH, Nagano Y, Choi IS, et al. Allelic alterations in well-differentiated neuroendocrine tumors (carcinoid tumors) identified by genome-wide single nucleotide polymorphism analysis and comparison with pancreatic endocrine tumors. Genes, Chromosomes and Cancer 2008;47:84-92.

33. Simbolo M, Vincentini C, Maffacini A, et al. Mutational and copy number asset of primary sporadic neuroendocrine tumors of the small intestine. Virchows Archiv 2018;473:709-717.

34. Banck MS, Kanwar R, Kulkarni AA, et al. The genomic landscape of small intestine neuroendocrine tumors. Journal of Clinical Investigation 2013;123:2502-2508.

35. Crona J, Gustavsson T, Norlén O, et al. Somatic mutations and genetic heterogeneity at the CDKN1B locus in small intestinal neuroendocrine tumors. Annals of Surgical Oncology 2015;22:S1428-S1435.

36. Shi Y, Qian ZR, Zhang S, et al. Cell cycle protein expression in neuroendocrine tumors. Pancreas 2017;46:1347-1353. 
37. Nieser M, Henopp T, Brix J, et al. Loss of chromosome 18 in neuroendocrine tumors of the small intestine: the enigma remains. Neuroendocrinology 2017;104:302-312.

38. Di Domenico A, Wiedmer T, Marinoni I et al. Genetic and epigenetic drivers of neuroendocrine tumors (NET). Endocrine-Related Cancer 2017;24:R315-R334.

39. Ruebel K, Leontovich A, Stilling G, et al. MicroRNA expression in ileal carcinoid tumours: downregulation of microRNA-133a with tumor progression. Modern Pathology 2009;23:367-375.

40. Finnerty BM, Gray KD, Moore MD, et al. Epigenetics of gastroenteropancreatic neuroendocrine tumors: a clinicopathologic perspective. World Journal of Gastrointestinal Oncology 2017;9:341.

41. Fotouhi O, Abdel Fahmideh M, Kjellman M, et al. Global hypomethylation and promoter methylation in small intestinal neuroendocrine tumors. Epigenetics 2014;9:987-997.

42. Ahuja N, Sharma AR, Baylin SB. Epigenetic therapeutics: a new weapon in the war against cancer. Annual Review of Medicine 2016;67:73-89.

43. Chakraborty C, Sharma AR, Sharma G, et al. Therapeutic miRNA and siRNA: moving from bench to clinic as next generation medicine. Molecular Therapy: Nucleic Acids 2017;8:132-143.

44. Cherny NI, Sullivan R, Dafni U et al. A standardised, generic, validated approach to stratify the magnitude of clinical benefit that can be anticipated from anti-cancer therapies: the European Society for Medical Oncology Magnitude of Clinical Benefit Scale (ESMO-MCBS). Ann Oncol 2015;26(8):1547-1573.

45. Schnipper LE, Davidson NE, Wollins DS et al. Updating the American Society of Clinical Oncology Value Framework: revisions and reflections in response to comments received. J Clin Oncol 2016;34:2925-2934.

46. Kiesewetter B, Raderer M, Steger GG et al. The European Society for Medical Oncology Magnitude of Clinical Benefit Scale in daily practice: a single institution, real-life experience at the Medical University of Vienna. ESMO Open 2016;1(4):e000066.

47. Del Paggio JC, Azariah B, Sullivan R, et al. Do Contemporary Randomized Controlled Trials Meet ESMO Thresholds for Meaningful Clinical Benefit? Ann Oncol. 2017;28(1):157-162.

48. Booth CM, Eisenhauer EA. Progression-free survival: meaningful or simply measurable? J Clin Oncol. 2012;30(10):1030-1033.

49. Pavel M, Oberg KE, Hainsworth JD et al. Everolimus plus octreotide long-acting release (LAR) for the treatment of advanced neuroendocrine tumors (NET) associated 
with carcinoid syndrome (RADIANT-2): updated overall survival results. Eur J Cancer 2013;49:S577.

50. Yao JC, Pavel M, Lombard-Bohas C et al. Everolimus for the treatment of advanced pancreatic neuroendocrine tumors: overall survival and circulating biomarkers from the randomized, phase III RADIANT-3 study. J Clin Oncol 2016;34(32):3906-3913

51. Yao JC, Fazio N, Singh S et al. Everolimus for the treatment of advanced, nonfunctional neuroendocrine tumours of the lung or gastrointestinal tract (RADIANT-4): a randomised, placebo-controlled, phase 3 study. Lancet 2016;387(10022):968-977.

52. de Baere T, Deschamps F, Tselikas L, et al. GEP-NETS update: Interventional radiology: role in the treatment of liver metastases from GEP-NETs. Eur J Endocrinol. 2015;172(4):R151-66.

53. Kennedy A, Bester L, Salem R, et al. Role of hepatic intra-arterial therapies in metastatic neuroendocrine tumours (NET): guidelines from the NET-liver-Metastases Consensus Conference. HPB (2015) 17:29-39

54. Korse T, Taal B, van Velthuysen $M$, et al. Incidence and survival of neuroendocrine tumours in the Netherlands according to histological grade: Experience of two decades of cancer registry. European Journal of Cancer 2013;49:1975-83.

55. Shah CP, Mramba LK, Bishnoi R,et al. Survival trends of metastatic small intestinal neuroendocrine tumor: a population-based analysis of SEER database. Journal of gastrointestinal oncology 2019;10:869-77.

56. Rossi RE, Massironi S, Spampatti MP, et al. Treatment of liver metastases in patients with digestive neuroendocrine tumors. Journal of Gastrointestinal Surgery : official journal of the Society for Surgery of the Alimentary Tract 2012;16:1981-92.

57. Modlin IM, Lye KD, Kidd M. A 5-decade analysis of 13,715 carcinoid tumors. Cancer. 2003;97(4):934-959.

58. Oleinikov K, Avniel-Polak S, Gross DJ, et al. Carcinoid Syndrome: Updates and Review of Current Therapy. Curr Treat Options Oncol. 2019;9;20(9):70.

59. Fisher AT, Titan AL, Foster DS, et al. Management of Ileal Neuroendocrine Tumors with Liver Metastases. Journal of gastrointestinal surgery : official journal of the Society for Surgery of the Alimentary Tract 2019.

60. Frilling A, Clift AK. Therapeutic strategies for neuroendocrine liver metastases. Cancer 2015;121:1172-86.

61. Elf AK, Andersson M, Henrikson O, et al. Radioembolization Versus Bland Embolization for Hepatic Metastases from Small Intestinal Neuroendocrine Tumors: Short-Term Results of a Randomized Clinical Trial. World J Surg. 2018;42(2):506-13. 
62. Chen JX, Rose S, White SB, et al. Embolotherapy for Neuroendocrine Tumor Liver Metastases: Prognostic Factors for Hepatic Progression-Free Survival and Overall Survival. CardioVascular and Interventional Radiology. 2016;40(1):69-80.

63. Bajetta E, Celio L, Platania M, et al. Single-institution series of earlystage Merkel cell carcinoma: long-term outcomes in 95 patients managed with surgery alone. Ann Surg Oncol. 2009;16:2985-2993.

64. Agelli M, Clegg LX. Epidemiology of primary Merkel cell carcinoma in the United States.J Am Acad Dermatol. 2003;49:832-841.

65. Andea AA, Coit DG, Amin B, Busam KJ. Merkel cell carcinoma: histologic features and prognosis. Cancer. 2008;113:2549-2558.

66. Sridharan VS. Merkel cell carcinoma: a population analysis on survival. J Natl Compr Cancer Netw. 2016;14:1247-1257.

67. Jabbour J, Cumming R, Scolyer RA, et al. Merkel cell carcinoma: assessing the effect of wide local excision, lymph node dissection, and radiotherapy on recurrence and survival in early-stage disease - results from a review of 82 consecutive cases diagnosed between 1992 and2004. Ann Surg Oncol. 2007;14:1943-1952.

68. Oliveria SA, Christos PJ, Halpern AC, et al. Evaluation of factors associated with skin self-examination. Cancer Epidemiol Biomarkers Prev. 1999;8:971-978.

69. Scoggins CR, Ross MI, Reintgen DS, et al. Gender-related differences in outcome for melanoma patients. Ann Surg. 2006;243:693-698.discussion 8-700.

70. Schrama D, Ugurel S, Becker JC. Merkel cell carcinoma: recent insights and new treatment options. Curr Opin Oncol. 2012;24:141-149.

71. Wang TS, Byrne PJ, Jacobs LK, et al. Merkel cell carcinoma: update and review. Semin Cutan Med. Surg. 2011;30:48-56.

72. Mojica P, Smith D, Ellenhorn JD. Adjuvant radiation therapy is associated with improved survival in Merkel cell carcinoma of the skin. J Clin Oncol. 2007;25:10431047.

73. Strom T, Carr M, Zager JS, et al. Radiation therapy is associated with improved outcomes in Merkel cell carcinoma. Ann Surg Oncol. 2016;23:3572-3578.

74. Ponte P, Vargas-Moniz J, Farricha V, et al. Merkel cell carcinoma: an unusual indication for isolated limb perfusion. Dermatol Online J 2008;14(7).

75. Moreno-Ramirez D, de la Cruz-Merino L, Ferrandiz L, et al. Isolated limb perfusion for malignant melanoma: systematic review on effectiveness and safety. Oncologist 2010;15(4):416-27.

76. Nghiem PT, Bhatia S, Lipson EJ, et al. PD-1 blockade with pembrolizumab in advanced Merkel-cell carcinoma. N Engl J Med 2016;374(26):2542-52. 
77. Kaufman HL, Russell J, Hamid O, et al. Avelumab in patients with chemotherapyrefractory metastatic Merkel cell carcinoma: a multicentre, single-group, open-label, phase 2 trial. Lancet Oncol 2016;17(10):1374-85.

78. Kaufman HL, Russell JS, Hamid O et al. Updated efficacy of avelumab in patients with previously treated metastatic Merkel cell carcinoma after $>/=1$ year of follow-up: JAVELIN Merkel 200, a phase 2 clinical trial. J Immunother Cancer 2018;6:7.

79. Schadendorf D, Lebbé C, zur Hausen A et al. Merkel cell carcinoma: Epidemiology, prognosis, therapy and unmet medical needs. European Journal of Cancer 2017;71:53-69.

80. Tetzlaff MT, Harms PW. Danger is only skin deep: aggressive epidermal carcinomas. An overview of the diagnosis, demographics, molecular-genetics, staging, prognostic biomarkers, and therapeutic advances in Merkel cell carcinoma. Mod Pathol. 2020;33(Suppl 1):42-55.

81. Fizazi K, Cojean I, Pignon JP et al. Normal serum neuron specific enolase (NSE) value after the first cycle of chemotherapy: an early predictor of complete response and survival in patients with small cell lung carcinoma. Cancer 1998;82:1049-1055.

82. Harmsma M, Schutte B, Ramaekers FC. Serum markers in small cell lung cancer: opportunities for improvement. Biochim Biophys Acta 2013;1836:255-272.

83. van Adrichem RC, Kamp K, Vandamme T et al. Serum neuron-specific enolase level is an independent predictor of overall survival in patients with gastroenteropancreatic neuroendocrine tumors. Ann Oncol 2016;27:746-747.

84. Paulson KG, Lewis CW, Redman MW et al. Viral oncoprotein antibodies as a marker for recurrence of Merkel cell carcinoma: A prospective validation study. Cancer 2017;123:1464-1474.

85. Erovic I, Erovic BM. Merkel cell carcinoma: the past, the present, and the future. J Skin Cancer. 2013;64:92-93. against MCPyV-encoded oncoproteins, hampering the use of these antibodies as a biomarker

86. Pavel M, Jann H, Prasad V, et al. NET Blood Transcript Analysis Defines the Crossing of the Clinical Rubicon: When Stable Disease Becomes Progressive.

Neuroendocrinology 2017;104:170-82.

87. Oberg K, Califano A, Strosberg JR, et al. A meta-analysis of the accuracy of a neuroendocrine tumor mRNA genomic biomarker (NETest) in blood. Annals of oncology : official journal of the European Society for Medical Oncology / ESMO 2020;31:202-12.

88. van Treijen MJC, Korse CM, van Leeuwaarde RS, et al. Blood Transcript Profiling for the Detection of Neuroendocrine Tumors: Results of a Large Independent Validation Study. Frontiers in endocrinology 2018;9:740. 
89. Modlin IM, Kidd M, Malczewska A, et al. The NETest: The Clinical Utility of Multigene Blood Analysis in the Diagnosis and Management of Neuroendocrine Tumors. Endocrinology and metabolism clinics of North America 2018;47:485-504.

90. Ahuja N, Sharma AR, Baylin SB. Epigenetic therapeutics: a new weapon in the war against cancer. Annual Review of Medicine 2016;67:73-89.

91. Chakraborty C, Sharma AR, Sharma G, et al. Therapeutic miRNA and siRNA: moving from bench to clinic as next generation medicine. Molecular Therapy: Nucleic Acids 2017;8:132-143.

92. Dayton T, Beumer J, Den Hartigh L, et al. Using Adult Stem-Cell Derived Organoids to Model Neuroendocrine Tumor Growth [Abstract] 15th Annual ENETS conference 2018 (2018)

91. Fernandez-Cuesta L, Foll M, Walter T, et al. The PanNENomics Project: A Call for an International Collaborative Effort Built on the Success of the LungNENomics Project [Abstract] 17th Annual ENETS Conference 2020 (2020)

93. Knepper TC, Montesion M, Russell JS, et al. The Genomic Landscape of Merkel Cell Carcinoma and Clinicogenomic Biomarkers of Response to Immune Checkpoint Inhibitor Therapy. Clin Cancer Res. 2019;25(19):5961-5971.

94. A Multicenter, Randomized, Double-Blinded, Placebo-Controlled, Phase 3 Trial of Adjuvant Avelumab (Anti-PDL-1 Antibody) in Merkel Cell Carcinoma Patients With Clinically Detected Lymph Node Metastases. Identifier: NCT03271372 https://clinicaltrials.gov/ct2/show/NCT0327137295.

95. Mehnert JM, Bergsland E, O'Neil BHet al. Pembrolizumab for the treatment of programmed death-ligand 1-positive advanced carcinoid or pancreatic neuroendocrine tumors: Results from the KEYNOTE-028 study. Cancer. 2020 Apr 22. doi: 10.1002/cncr.32883.

96. Lybaert W, Vandamme T, Boons G, et al. Highlights of the 2018 Annual European Neuroendocrine Tumor Society (ENETS) Congress. Belg J Med Oncol 2018:12(5):252262 



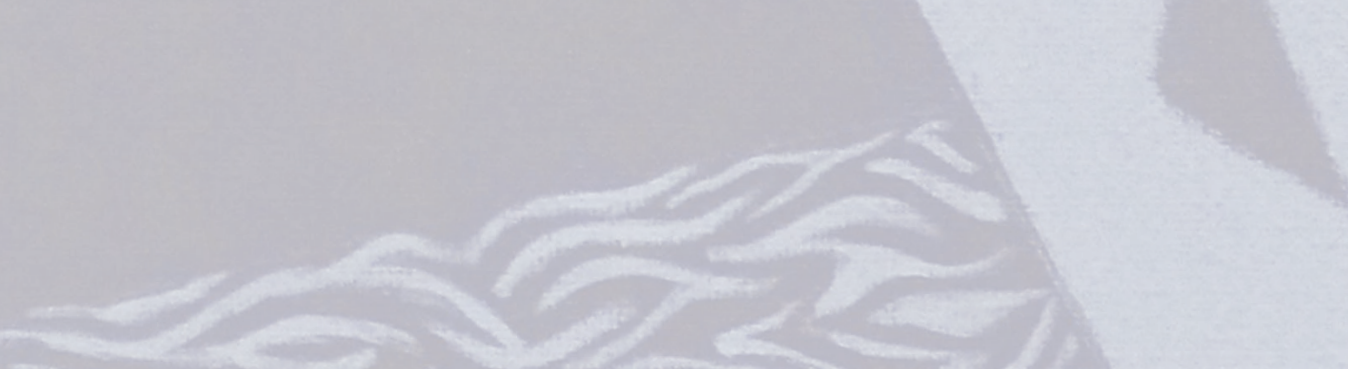

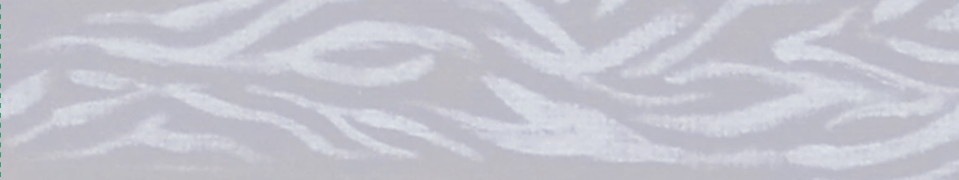




\section{Appendices}





\section{Dutch summary | Nederlandstalige samenvatting}

Neuroendocriene neoplasieën (NEN) zijn zeldzame maligniteiten die ontstaan vanuit neuroendocriene cellen. Deze cellen zitten verspreid door het gehele lichaam. Ze worden vaak aangehaald ware het eenzelfde groep tumoren, met name wanneer het NEN betreffen van het maag-darm kanaal. Alhoewel ze histologisch (onder de microscoop) erg op elkaar kunnen lijken, verschillen ze per lokalisatie aanzienlijk ten aanzien van de hormonen die ze produceren, het moleculair profiel en het ziektebeloop. Deze verschillen hebben grote invloed op de prognose en de behandeling van patiënten met NEN. Helaas heeft het relatief lang geduurd voordat dit onderkend werd en richt veel onderzoek zich op neuroendocriene tumoren van het maag-darm stelsel (GEP-NETs) in het geheel, en in het geval van Merkel cel carcinoom (MCC) binnen een groep van zeldzamen tumoren van de huid.

Dat de verschillen tussen NEN lange tijd onderbelicht zijn geweest heeft te maken met verschillende oorzaken. De complexe geschiedenis ten aanzien van de naamgeving en classificatie speelt hierin een grote rol. De eerste NEN werd in 1867 aangetoond in de dunne darm. De gevonden tumor leek niet op de reeds bekende vorm van darmkanker, welke ontstaat vanuit kliervormende cellen, het zogenaamde adenocarcinoom. In 1907 werden deze nieuwe tumoren dan ook carcinoïd ('carcinoomachtigen') genoemd waarbij werd aangenomen dat dit zogenaamde 'goedaardige' tumoren waren. In 1938 werden deze tumoren toegeschreven aan het endocriene (hormoon producerende) systeem, dat door het gehele lichaam verspreid zit. Alle tumoren die ontstonden vanuit dit systeem werden vervolgens carcinoïden genoemd, waarbij neuroendocriene tumoren van de dunne darm werden gezien als de klassieke carcinoïden. In 1963 werden carcinoïd tumoren grof opgedeeld op basis van embryologische origine in voor-darm (waaronder het pulmonale systeem, slokdarm, maag, twaalfvingerige darm, alvleesklier, eierstokken), midden-darm (verschillende delen van de dunne darm, blindedarm en opstijgende deel van de dikke darm) en achter-darm (overige delen van de dikke darm). Daarnaast werd duidelijk dat sommige van deze tumoren zich agressiever gedroegen dan anderen. Daarom werden neuroendocriene tumoren ingedeeld in graden op basis van specifieke kenmerken van het weefselonderzoek. In 2000 werd de term carcinoïd grotendeels verlaten. In de jaren erna werd de gradering verder aangepast met onder andere de Ki-67 index (groeisnelheid) en de mitotische index (mate van celdeling). De laatste aanpassingen dateren uit 2017 en 2019 waardoor we op dit moment NEN 
indelen op basis van lokalisatie en graad 1, 2 of 3 neuroendocriene tumor (NET) of een neuroendocrien carcinoom (NEC).

Daarnaast heeft de relatieve zeldzaamheid van NEN bijgedragen aan een gebrek aan aandacht voor de verschillende vormen van NEN in wetenschappelijk onderzoek. Voor een heterogene (gevarieerde) groep van tumoren, zoals NEN, zijn er grote patiëntpopulaties nodig om met de verschillende eigenschappen statistisch rekening te kunnen houden. Desalniettemin zijn onderzoeken vaak gebaseerd op kleine cohorten of nationale databases met weinig gedetailleerde informatie vanwege de zeldzaamheid van de aandoening.

Het huidige onderzoek en de behandeling van oncologische patiënten richt zich steeds meer op gepersonaliseerde zorg. Dat is zorg toegepast op de specifieke kenmerken van de tumor en de patiënt om tot betere kankerzorg te komen. Het Antoni van Leeuwenhoek - Nederlands Kanker Instituut en het UMC Utrecht vormen tezamen een, door het European Neuroendocrine Tumour Society (ENETS) erkend centrum van expertise. Patiënten hebben hier de beschikking over een multidisciplinair team met veel ervaring in het behandelen van de verschillende vormen van NEN. Bovendien zijn alle behandelingen volgens de nieuwste inzichten beschikbaar. De grote patiëntenpopulatie biedt mogelijkheden voor het doen van onderzoek naar de prognose en effecten van behandeling, rekening houdend met de specifieke typen van NEN. In dit proefschrift wordt de prognose en behandeling van patiënten met een NET van de dunne darm (SI-NET) en NEC van de huid, ook wel MCC genaamd, bestudeerd.

- NET van de dunne darm

Van de GEP-NET zijn SI-NET de op één na meest voorkomende tumoren. SI-NET is tevens de meest voorkomende vorm van kanker van de dunne darm. De incidentie (het aantal nieuwe diagnoses per jaar) is in Nederland de afgelopen jaren toegenomen tot 6.61 patiënten per 100.000 personen in 2011. De stijging is grotendeels te verklaren door een meer uniforme definiëring en verbeterde diagnostische mogelijkheden. Patiënten met een NEN kunnen klachten ervaren door de productie van hormonen door de tumorcellen. We noemen dit dan een functionele tumor. In patiënten met een SI-NET worden deze klachten het carcinoïd syndroom genoemd, waarbij de klachten bestaan uit diarree, flushing (blozen), een piepende ademhaling en in sommige gevallen ook een verminderde hartklepfunctie, 
wat kan leiden tot carcinoïd hartziekte. De enige curatieve behandeling voor patiënten met een SI-NET bestaat uit een operatieve verwijdering van de tumor(en). Ten tijde van de diagnose heeft reeds $40 \%$ van de patiënten metastasen, veelal naar de lever. Een minderheid van deze patiënten (20-30\%) komt in aanmerking voor een operatie. Niet-curatieve opties van behandeling zijn, de reeds uitgebreid onderzochte, somatostatine analogen. Daarnaast hebben recente studies het effect aangetoond van peptide receptor radionuclide therapie (ookwel PRRT, een nucleaire therapie) en chemotherapie. Trans-arteriële embolisatie (TAE) en radioembolisatie (SIRT) zijn behandelingen die zich richten op levermetastasen. Ze maken gebruik van de vaatvoorziening van de lever en emboliseren de vaattoevoer naar de metastasen, waarbij SIRT gebruik maakt van radioactieve partikels. Alhoewel deze technieken reeds gebruikt worden in de klinische praktijk is er weinig vergelijkend onderzoek gedaan naar de effectiviteit van deze behandelingen bij patiënten met NET tumoren.

Ondanks dat een groot deel van de patiënten zich presenteert met metastasen, bestaat er een relatief gunstige overleving. De 5-jaars overleving van patiënten met operabele ziekte is ongeveer $75 \%$ en in het geval van onbehandelde levermetastasen zien we een 5-jaars overleving van ongeveer 25-50\%. Eerder geïdentificeerde prognostische factoren zijn uitgebreidheid van de ziekte, gradering en aanwezigheid van levermetastasen. Alhoewel er prognostische data beschikbaar is van cohorten van patiënten met een SI-NET, is er behoefte aan een individuele schatting van prognose om behandelingsstrategieën te bepalen.

\section{- Merkel cel carcinoom}

MCC is een NEC van de huid. De eerste MCC werd in 1972 beschreven waarbij de tumorcellen overeenkomsten vertoonden met Merkel cellen die in de huid voorkomen. Recent onderzoek laat echter zien dat deze maligniteit mogelijk zijn oorsprong vindt in andere cellen van de huid, maar de naamgeving is vooralsnog onveranderd. MCC komt met name voor bij oudere patiënten. De tumor zit vaak in het hoofd-hals gebied en op de ledematen. Deze gebieden worden blootgesteld aan ultraviolet straling waardoor er gedacht wordt dat dit een rol speelt in de ontwikkeling van MCC. Andere risicofactoren zijn het gebruik van immunosuppressiva en de aanwezigheid van het Merkel cel polyoma virus.

MCC staat bekend om zijn agressieve ziektebeloop. Ten tijde van de diagnose heeft reeds $25 \%$ metastasen in de lymfeklieren. Locoregionale terugkeer of progressie van 
de ziekte wordt gezien in 60\% van de patiënten, vaak binnen twee jaar. De 5-jaars overleving varieert tussen de 30-60\%. De grootte van de primaire tumor en de aanwezigheid van lymfekliermetastasen zijn in eerder onderzoek geassocieerd met een slechtere overleving.

De slechte overleving en grote kans op terugkeer van de ziekte legitimeert de vaak agressieve behandelingsstrategie. In geval van locoregionale ziekte volgt, indien mogelijk, operatieve verwijdering en vaak postoperatieve bestraling. Chemotherapie wordt enkel toegepast bij metastasen op afstand. Hierbij wordt vaak een respons gezien, maar deze is meestal van korte duur. Recent werd immunotherapie geïntroduceerd voor patiënten met gemetastaseerd MCC. Door de implementatie van deze nieuwe therapie en de daarbij verbeterde overleving is er een toegenomen behoefte aan een serum biomarker (bloedwaarde die iets zegt over ziektestatus).

- Deel I: Prognose in neuroendocriene neoplasieën

Het eerste deel van het proefschrift richt zich op de bestudering van prognose van patiënten met een SI-NET en MCC. Het voorspellen van prognose van patiënten met een SI-NET is uitdagend doordat dit ziektebeeld een gevarieerd ziektebeloop kent. Hierdoor is het lastig om voor een afzonderlijke patiënt een prognose te geven, rekening houdend met specifieke patiënt- en ziektekenmerken. Een manier om een individuele prognose te schatten is door middel van een nomogram. In 2010 is op basis van klinische- en tumorkarakteristieken een nomogram ontwikkeld voor patiënten met een SI-NET. In hoofdstuk 2 is dit nomogram gevalideerd in 400 patiënten uit ons centrum. In onze populatie kon het nomogram patiënten onderscheiden in een laag-, medium- en hoog risicogroep met een daarbij behorende geschatte ziektespecifieke overleving. Het nomogram onderschatte voor de meeste patiënten de overleving. Dit laat zien dat het nomogram verbetering behoeft. In onze populatie hebben we verder onderzocht welk factoren van invloed zijn op de ziektespecifieke overleving. Het bleek dat leeftijd, een WHO perfomance status van 2 (dit geeft het niveau van functioneren van een patiënt weer), metastasen waarbij de primaire tumor niet gevonden kon worden, Ki-67 index $\geq 10 \%$, verhoogd Chromogranine A (biomarker in het bloed) en verhoogde leverwaarden de overleving verlagen. Het nomogram aanvullen met nieuwe (moleculaire) biomarkers of prognostische factoren zou het schatten van de overleving kunnen verbeteren.

De afgelopen jaren is de moleculaire achtergrond van SI-NETs steeds meer in kaart gebracht. In hoofdstuk 3 is een literatuurstudie verricht naar moleculaire kenmerken 
van SI-NETs en welke van deze kenmerken mogelijk een invloed hebben op de prognose. Onze studie toonde dat SI-NETs over het algemeen weinig genetische mutaties (mutaties in codering van de genen) lieten zien, terwijl epigenetische veranderingen (veranderingen in de functie van de genen) veel vaker voorkwamen. Helaas hebben maar 16 van de 35 geïncludeerde studies een prognostische analyse uitgevoerd, waarbij acht studies daadwerkelijk een associatie konden aantonen met prognose. Verlies van heterogeniteit van chromosoom 18, geclusterde activerende mutaties van chromosoom 4, 5, 7, 14, 20p en het SRC gen (een proto-oncogen) en verminderde expressie van RASSF1 en P16 zijn geassocieerd met een verminderde overleving.

De (epi)genetische afwijkingen vervullen nog geen rol in de dagelijkse klinische praktijk om een prognose te schatten. De genetische mutaties in SI-NETs zijn over het algemeen minder geschikt voor het ontwikkelen van eventuele nieuwe behandelingen. Daarentegen bieden de geïdentificeerde epigenetische veranderingen mogelijke opties om nieuwe therapieën te ontwikkelen.

In hoofdstuk 4 is een prognostische studie uitgevoerd in een cohort van 351 patiënten met MCC. Dit is het op één na grootste cohort tot nu toe. Ondanks dat de algehele overleving laag was (5-jaars overleving van 58\%), bleek er sprake te zijn van een relatief laag ziektespecifieke sterfte (5-jaars sterfte van 22\%). Dat betekent dat de (vaak oudere) patiënten met MCC veelal aan andere oorzaken komen te overlijden. Dit is belangrijke informatie wanneer er tussen mogelijk agressieve behandelingsstrategieën gekozen moet worden. Postoperatieve bestraling verlaagde de kans op terugkeer van de ziekte. Het mannelijk geslacht en de aanwezigheid van metastasen in de lymfeklieren verhoogden de ziektespecifieke sterfte.

Tot op heden is er voor patiënten met MCC geen accurate biomarker geïdentificeerd die het effect van therapie kan meten of de prognose kan voorspellen. Neuronspecific enolase (NSE) kan in het bloed worden gemeten en wordt al in andere maligniteiten gebruikt als een biomarker. De rol van NSE voor patiënten met MCC is vooralsnog onduidelijk. In hoofdstuk $\mathbf{5}$ is onderzocht of NSE gebruikt kan worden om informatie te geven over prognose en effectiviteit van therapie. Hiervoor was data beschikbaar van 84 patiënten met 565 bloedafnames. NSE correleerde met uitgebreidheid van de ziekte. Een afkapwaarde van $18.2 \mathrm{ng} / \mathrm{ml}$ bleek klinisch het meest waardevol om terugkeer of progressie van de ziekte uit te sluiten. Ook was NSE in staat om patiënten die immunotherapie kregen te onderscheiden in 
responders en non-responders. NSE zou daarmee een waardevolle biomarker kunnen worden voor patiënten met MCC.

- Deel 2: Behandeling van patiënten met neuroendocriene neoplasieën

Het tweede deel van het proefschrift richt zich op de effecten van verschillende therapieën voor patiënten met een GEP-NET en MCC. In hoofdstuk 6 worden verschillende systemische therapieën voor patiënten met een GEP-NET geëvalueerd middels twee tools: 'the European Society for Medical Oncology Magnitude of Clinical Benefit Scale' (ESMO-MCBS) en 'the American Society of Clinical Oncology Framework' (ASCO-F). Hiervoor werden 35 trials (therapeutische onderzoeken) onderzocht. Enkel zes van de 35 trials voldeden aan de evaluatiecriteria van de ESMO-MCBS, resulterend in een score van 2 of 3 . Negen trials voldeden aan de evaluatie criteria van de ASCO-F, resulterend in scores van 37.6 tot 57.4. Dit zijn relatief lage scores. De hoogste scores werden behaald door de RADIANT-3 trial waarin everolimus (een therapie gericht op de mTOR receptor) werd vergeleken met een placebo en de NETTER-1 trial waarin 177Lu-dotatate in combinatie met octreotide LAR werd vergeleken met enkel octreotide LAR (oftewel PRRT vergeleken met een somatostatine analoog). De lage scores kunnen deels worden verklaard doordat veel studies als eindpunt duur tot progressie hanteerden in plaats van (ziektespecifieke) overleving. Daarnaast werden vaak heterogene patiëntpopulaties onderzocht, waarbij geen analyses werden verricht voor de verschillende tumorgroepen. Het is mogelijk dat in deze populaties een behandeling beter werkt voor een NET van de pancreas dan voor een SI-NET, en dit de resultaten beïnvloedde.

De aanwezigheid van levermetastasen is een prognostische factor die de overleving van patiënten met een NET sterk beïnvloedt. Daarnaast zorgt het vaak ook voor een zware ziektelast. Therapieën die gericht zijn op het behandelen van levermetastasen zijn daarom van belang voor zowel het verbeteren van de prognose als kwaliteit van leven. In hoofdstuk 7 werden TAE en SIRT met elkaar vergeleken ten aanzien van toxiciteit, respons en overleving. Er werd een aparte analyse verricht voor patiënten met een SI-NET en een NET van de pancreas. In een cohort van 86 patiënten en 130 procedures toonde onze studie aan dat zowel TAE als SIRT veilige en effectieve behandelingen zijn voor patiënten met levermetastasen. Desalniettemin hadden de therapieën verschillende bijwerkingsprofielen en leken functionele klachten meer af 
te nemen na TAE. Met betrekking tot het effect op overleving werd geen verschil aangetoond, al werd er een trend gezien voor een betere overleving na SIRT voor patiënten met een NET van de pancreas.

Voor patiënten met uitgebreide locoregionale ziekte door een MCC bestaat geen gestandaardiseerde behandeling. In hoofdstuk 8 werd een cohort van tien patiënten onderzocht die isolated limb perfusion (ILP) ondergingen. Door middel van ILP wordt een hoge dosis chemotherapie aan een extremiteit toegediend. Na de behandeling werd bij geen enkele patiënt symptomen gezien van systemische toxiciteit. Ernstige locoregionale toxiciteit trad op bij twee patiënten. Van acht patiënten kon de respons geëvalueerd worden. Een respons trad op in $87.5 \%$ waarbij in $62.5 \%$ sprake was van een complete respons. Bij twee patiënten was er sprake van een langdurige respons (53 en 71 maanden). De studie toonde aan dat ILP een effectieve behandeling is voor patiënten met uitgebreide locoregionale ziekte door MCC. Zorgvuldige selectie van de vaak oudere patiënten is belangrijk vanwege de hoge kans op toxiciteit.

Concluderend werden met dit proefschrift de eerste stappen gezet om de prognose en behandeling van patiënten met een NEN meer tumorspecifiek te benaderen. De uitdaging bestaat uit het verbeteren van de geïndividualiseerde schatting van prognose voor patiënten met een SI-NET. Moleculaire eigenschappen of nieuw ontwikkelde (moleculaire) biomarkers zouden onderzocht kunnen worden in combinatie met een nomogram gebaseerd op klinische kenmerken. Wellicht bieden de epigenetische afwijkingen tevens aanknopingspunten voor het ontwikkelen van nieuwe therapieën. Voor patiënten met MCC is het vooral belangrijk om de ziektespecifieke overleving mee te nemen bij het bepalen van een behandelingsstrategie. Daarnaast is de verwachting dat immunotherapie steeds vaker toegepast zal worden, bijvoorbeeld voorafgaand aan een operatie. Het is van belang dat NSE ook in deze setting geëvalueerd zal worden. 



\section{Contributing authors and affiliations}

A.C.J. van Akkooi, MD, PhD

Department of Surgical Oncology

Netherlands Cancer Institute, Amsterdam, the Netherlands

J.G. van den Berg, MD, PhD

Department of Pathology

Netherlands Cancer Institute, Amsterdam, the Netherlands

E. Bertolli, MD, PhD

Skin Cancer Department

AC Camargo Cancer Center, Sao Paulo, Brazil

A.J.A.T. Braat, MD, PhD

Department of Radiology and Nuclear Medicine

University Medical Center Utrecht, the Netherlands

E.C.H. Breekveldt,

Department of Medical Oncology

Netherlands Cancer Institute, Amsterdam, the Netherlands

D.J. Grünhagen, $M D, P h D$

Departement of Surgical Oncology

Erasmus MC Cancer Institute, Rotterdam, The Netherlands

W. de Herder, MD, PhD

Department of Endocrinology

Erasmus Medical Center, Rotterdam, the Netherlands

L. de Hosson, MD, PhD

Department of Medical Oncology

University Medical Center Groningen, the Netherlands

W.M.C. Klop, Md, PhD

Department of Head and Neck Surgery

Netherlands Cancer Institute, the Netherlands 
Appendices

C.M. Korse, PhD

Department of Clinical Chemistry

Netherlands Cancer Institute, the Netherlands

H.J. Klumpen, MD, PhD

Department of Medical Oncology

Academic Medical Center, Amsterdam, the Netherlands

K. Kuhlmann, MD, PhD

Department of Surgical Oncology

Netherlands Cancer Institute, Amsterdam, the Netherlands

M.G.E.H. Lam, MD, PhD

Department of Radiology and Nuclear Medicine

University Medical Center Utrecht, the Netherlands

S. Levy, MD

Department of Medical Oncology

Netherlands Cancer Institute, Amsterdam, the Netherlands

M.F. Madu, MD, PhD

Department of Surgical Oncology

Netherlands Cancer Institute, Amsterdam, the Netherlands

W. Prevoo, MD, PhD

Department of Radiology

Netherlands Cancer Institute, Amsterdam, the Netherlands

K.G. Samsom, MD

Department of Medical Oncology and Pathology

Netherlands Cancer Institute, Amsterdam, the Netherlands

M.E.T. Tesselaar, MD, PhD

Department of Medical Oncology

Netherlands Cancer Institute, Amsterdam, the Netherlands 
G.D. Valk, MD, PhD

Department of Endocrine Oncology

University Medical Center Utrecht, the Netherlands.

W.H.M. Verbeek, MD, PhD

Department of Gastroenterology

Netherlands Cancer Institute, Amsterdam, the Netherlands

J. Verdult, MD, PhD

Department of Radiology and Nuclear Medicine

University Medical Center Utrecht, the Netherlands

C. Verhoef, MD, PhD

Departement of Surgical Oncology

Erasmus MC Cancer Institute, Rotterdam, The Netherlands

M.R. Vriens, MD, PhD

Department of Surgical Oncology and Endocrine Surgery

University Medical Center Utrecht, the Netherlands

Y. Schuller, MD, PhD

Department of Medical Oncology

Academic Medical Center, Amsterdam, the Netherlands

W. Zandee, MD, PhD

Department of Endocrinology

Erasmus Medical Center, Rotterdam, the Netherlands

A. Walenkamp, MD, PhD

Department of Medical Oncology

University Medical Center Groningen, the Netherlands 



\section{List of publications}

Comparison of transarterial bland embolization and selective internal radiation therapy in patients with metastatic neuroendocrine tumours

L.M. van Veenendaal, K.G. Samsom, A.J.A.T. Braat, J. Verdult, W. Prevoo, W.H. Verbeek, G.D. Valk, M.R. Vriens, M.G.E.H. Lam, M.E.T. Tesselaar, submitted for publication

Cancer driver mutations occur frequently in metastases of sporadic welldifferentiated neuroendocrine tumours of the small intestine K.G. Samsom, S. Levy* L.M. van Veenendaal*, P. Roepman, L.L. Kodach, N.Steeghs, G.D. Valk, W.M. Dercksen, K.F.D. Kuhlmann, W.H.M. Verbeek, G.A. Meijer, M.E.T. Tesselaar, J.G. van den Berg, submitted for publication, * both authors contributed equally

Survival in patients with neuroendocrine tumour of the small intestine: nomogram validation and predictors of survival L.M. van Veenendaal*, S. Levy*, C.M. Korse, W.H.M. Verbeek, M.R. Vriens, K. Kuhlmann, J.G. van den Berg, G.D. Valk, M.E.T. Tesselaar, Journal of Clinical Medicine, $2020 *$ both authors contributed equally

The clinical utility of neuron-specific enolase serum levels as a biomarker for Merkel cell carcinoma

L.M. van Veenendaal, E. Bertolli, C.M. Korse, W.M.C. Klop, M.E.T. Tesselaar, A.C.J. van Akkooi, Annals of Surgical Oncology, 2020

ASO author reflections: neuron-specific enolase as a valuable biomarker for patients with Merkel cell carcinoma in the era of immunotherapy

L.M. van Veenendaal, A.C.J. van Akkooi, Annals of Surgical Oncology, 2020

Molecular prognostic factors in small-intestinal neuroendocrine tumours

K.G. Samsom, L.M. van Veenendaal, G.D. Valk, M.R. Vriens, M.E.T. Tesselaar, J.G. van den Berg, Endocrine Connections, 2019 
Appendices

Letter re: Efficacy of isolated limb perfusion in patients with Merkel cell carcinoma: a multicenter experience

L.M. van Veenendaal, M.F. Madu, M.E.T. Tesselaar, C. Verhoef, D.J. Grünhagen, A.J.C. van Akkooi, European Journal of Surgical Oncology, 2019

Merkel cell carcinoma: clinical outcome and prognostic factors in 351 patients L.M. van Veenendaal, A.C.J. van Akkooi, C. Verhoef, D.J. Grünhagen, W.M.C. Klop, G.D. Valk, M.E.T. Tesselaar, Journal of Surgical Oncology, 2018

Clinical benefit of systemic treatment in patients with advanced pancreatic and gastro-intestinal neuroendocrine tumours according to ESMO-MCBS and ASCO framework

L. de Hosson, L.M. van Veenendaal, Y. Schuller, W. Zandee, W. de Herder, M. Tesselaar, H.J. Klumpen, A. Walenkamp, Annals of Oncology, 2017

Concurrent Merkel cell carcinoma and melanoma in individual patients presents a treatment challenge: a case series M.F. Madu, L.M. van Veenendaal, B. van de Wiel, M.E.T. Tesselaar, A.C.J. van Akkooi, Clinical Skin Cancer, 2017

Efficacy of isolated limb Perfusion in patients with Merkel cell carcinoma: a multicenter experience

L.M. van Veenendaal*, M.F. Madu*, M.E.T. Tesselaar, C. Verhoef, D.J. Grünhagen, A.C.J. van Akkooi, European Journal of Surgical Oncology, $2017 *$ both authors contributed equally

Recommendations for radioembolisation after liver surgery using yttrium-90 resin microspheres based on a survey of an international expert panel M. Samim, L.M. van Veenendaal. M.N.G.J.A. Braat, A.F. van den Hoven, R. van Hilligersberg, B. Sangro, Y.H. Kao, D. Liu, J.D. Louie, D.Y. Sze, S.C. Rose, D.B. Brown, H. Ahmadzadehfar, E. Kim, M.A.A.J. van den Bosch, M.G.E.H. Lam, European Radiology, 2017

A painful finger as first sign of a malignancy

L.M. van Veenendaal, G. de Klerk, D. van der Velde, Geriatric Orthopaedic Surgery \& Rehabilitation, 2014 




\section{Review committee}

Prof. dr. R. Goldschmeding

Department of Pathology

University Medical Center Utrecht

Utrecht, the Netherlands

Prof. dr. J.B.A.G. Haanen

Department of Clinical Oncology

Leiden University Medical Center

Leiden, the Netherlands

Prof. dr. I.Q. Molenaar

Department of Surgical Oncology

University Medical Center Utrecht

Utrecht, the Netherlands

Prof. dr. F.P. Vleggaar

Department of Gastroenterology and Hepatology

University Medical Center Utrecht

Utrecht, the Netherlands

dr. A.C.J. van Akkooi

Department of Surgical Oncology

Antoni van Leeuwenhoek - Netherlands Cancer Institute

Amsterdam, the Netherlands 



\section{Acknowledgements | Dankwoord}

Traditiegetrouw begint dit hoofdstuk met het vermelden dat het dankwoord het best gelezen deel van een proefschrift is. Volkomen terecht! Veel mensen hebben (inhoudelijk danwel moreel) aan dit proefschrift bijdragen en een aantal wil ik in het bijzonder bedanken.

Prof. dr. Vriens, beste Menno. Allereerst veel dank voor de kans om te promoveren bij de neuroendocriene researchgroep. Zonder jou zou dit boekje er simpelweg niet zijn geweest. Jij hebt me tijdens mijn semi-arts stage de kans geboden om onderzoek te doen op de manier waarop ik hoopte: in een dedicated en ambitieuze groep die klinisch relevant en kwalitatief goed onderzoek wilt voortbrengen. Gedurende mijn promotie gaf je me vertrouwen om zelf oplossingen voor problemen te vinden en zorgde voor focus(!), met name als ik te veel projecten wilde doen. Ik heb hierdoor enorm veel van dit promotietraject kunnen leren. Ik bewonder hoe jij mensen weet te verbinden. Dit heeft onder andere geleid tot de samenwerking met het Hubrecht Instituut, met als doel organoids van NET tumoren te kweken, een project van wereldformaat. Maar bovenal wil ik je bedanken voor jouw aanstekelijke energie en jouw visie op onderzoek, opleiding, chirurgie en het goede leven. Dit is onontbeerlijk gedurende een, bij tijd en wijle, stug proces als een promotietraject en voor een dokter met (chirurgische) toekomstplannen. Dank!

Prof. dr. Valk, beste Gerlof, ik weet nog goed hoe ik mijn eerste gesprek met jou had. Je staat voor klinisch relevant onderzoek, onderzoek dat ertoe doet voor de patiënt. Simpele vragen zijn goede vragen en 'rubbish in = rubbish out'. Tevens vertelde je mij meteen dat database onderzoek een taaie kluif kan zijn. Ik kan dit allemaal bevestigen. Echter, met jouw kennis van database onderzoek is een promovendus in goede handen. Of het de MEN database of de NEN database betreft, jij weet het maximale eruit te halen. Dank voor jouw vertrouwen en begeleiding. 
Dr. Tesselaar, beste Margot, als eerste promovenda van het gezamenlijke NET/NECcentrum mocht ik in het $A v L$ een plekje zoeken. Jij hebt mij vervolgens wegwijs gemaakt in de wereld van neuroendocriene tumoren, toen nog een onontgonnen terrein voor mij. Daarvoor maakte je wekelijks tijd. Je was streng als het moest en was er ook op lastige momenten. Ik waardeer dat enorm. Dankzij jou is het NEN onderzoek een (snel)trein geworden waar steeds meer mensen op springen. Ik hoop dat je trots bent.

Dr. Korse, beste Tiny, enorm veel dank voor jouw betrokkenheid bij mij als jonge onderzoeker en als persoon. Jouw integriteit is inspirerend. Ik waardeer het enorm dat jouw deur en mailbox altijd open staat voor advies.

Dr. van Akkooi, beste Alexander, het Merkel cel onderzoek is onverwacht een aanzienlijk onderdeel van dit proefschrift geworden. Het was een groot plezier met je samen te werken en me in de wereld van het Merkel cel onderzoek te storten. Het is indrukwekkend om te zien hoe jullie de zorg voor patiënten met Merkel cel carcinoom weten te verbeteren. Veel dank voor het plaatsnemen in de leescommissie.

Geachte leden van de leescommissie, prof. dr. R. Goldschmeding, prof. dr. J.B.A.G. Haanen, prof. dr. I.Q. Molenaar, prof. dr. F.P. Vleggaar, veel dank voor uw tijd en inspanning om dit proefschrift kritisch te beoordelen. Ik kijk ernaar uit om er met u van gedachten over te wisselen.

Geachte co-auteurs, dank voor de prettige samenwerking en jullie kritische bijdrage aan de studies en manuscripten.

Geachte OOA commissie Koert Kuhlmann, Monique van Leerdam, Michelle Versleijen, dank voor de begeleiding en het monitoren van de voortgang van mijn promotie naast jullie drukke klinische werkzaamheden en eigen onderzoeksprojecten. 
De groeiende endoresearch groep bestaande uit gepassioneerde chirurgen, endocrinologen, oncologen, nucleair geneeskundigen en pathologen haalt het beste in elkaar naar boven. Dank dat ik hiervan deel uit mocht maken. In het bijzonder wil ik mede-onderzoekers: Rachel van Leeuwaarde, Mark van Treijen, Sjoerd Nell, Wessel Vorselaars en Dirk Jan van Beek bedanken voor jullie inbreng tijdens de besprekingen, borrels en koffietjes. Rachel, wat een heerlijk congres in Boston hebben we gehad. Kris Samsom, je bent een talent en ik heb enorm veel respect hoe jij de pathologie en (epi)genetica jou eigen hebt kunnen maken. Sonja Levy, een betere opvolger kan Margot zich niet wensen. Ook dank voor Emilie Breekveldt, als student heb jij je kranig ingezet voor de NEN database. Het was heel fijn samenwerken.

Overige leden van het NET-team: Wieke Verbeek, Lisette Saveur, Nick van der Meij, dankzij jullie uitstekende zorg voor de NET patiënten bestaat er een groeiende database waarmee onderzoek kan worden verricht.

Patiënten en NET stichting, dit onderzoek is voor jullie en dankzij jullie. Carmen Kleinegris; jouw stem en vechtlust zorgen ervoor dat NET patiënten gehoord worden.

AvL promovendi, achter een computer zitten is niet mijn sterkste kant maar dankzij jullie werd het een stuk draaglijker. Met name kamergenootjes Marieke van der Noorda, Lisette Rozeman en nog eventjes Ariane van Loevezijn, dank voor het aanhoren van het commentaar op de Achterklap-nieuwtjes en de muziek die persé over de boxen moest. Arthur Kooyer, dank voor de korte pauzes om buiten even een frisse neus te halen;). Dank UMCU promovendi voor het lenen van jullie werkplekjes en voor de uitputtende wintersport.

Onderzoek doen is onmogelijk zonder secretaresses als Astrid Jonk en Romy Liesdek! 
Appendices

Tevens wil ik de stafleden, AIOS, ANIOS, VS-ers, PA-ers, verpleegkundigen en poliassistenten van de afdeling Heelkunde van het Antoniusziekenhuis bedanken. Ook Jolanda mag niet missen. Bij jullie heb ik mijn eerste stappen mogen zetten als ANIOS. Dank voor jullie warme welkom en dat jullie van mij een echte dokter hebben gemaakt. Ik geniet ervan!

Onderstaande personen hebben geen directe relatie met onderzoeksgroepen, studies of manuscripten. Integendeel zelfs, zonder hen zou het proefschrift wellicht sneller zijn afgerond. Maar ondanks mijn frequente afwezigheid door deadlines, syntaxen herhalen, checken, nog een keer checken en revisies, hebben ze altijd bijzondere interesse weten te tonen in mijn promotietraject. Bovendien gaf elke afleiding tevens hernieuwde energie om ertegen aan te gaan.

Carlijn, Emma, Frederike, Jantine en Lorette. 'T cluppie gaat al een tijdje mee, hoeveel nakomelingen en grijze haren zijn we inmiddels rijker? Dank voor jullie niet-aflatende interesse in mijn promotie, ook al kon ik hierdoor vaak niet aanwezig zijn. Ik zal me nu weer vaker melden, can't wait! Dit blijft t einde!

Clasine, Roos en Jasmijn, wij hebben elkaar in het vreemdste klasje ooit leren kennen. Vooraf afspreken blijkt onmogelijk, maar 'iemand morgen iets te doen?' lijkt wonderwel beter te werken. Dank dat het nog steeds lukt!

Lydia, Michelle en Quirine, allen apart lopen jullie al heel wat jaartjes mee. Kwaliteit in plaats van kwantiteit is van toepassing op hoe vaak we elkaar zien. Jullie zijn dierbare vrienden.

Bergerac crew, wat zouden de zomers en feestjes saai zijn zonder jullie. Bizar hoe je na zo'n slopende vakantie er toch weer tegenaan kan. Ze werden natuurlijk mede mogelijk gemaakt door de Bolkensteintjes met speciale vermelding voor Sanne, Bert en Carina, warmere en gastvrijere mensen zijn niet te bedenken. Sanne, dank voor 
jouw woorduitvindstoornissen, dat maakt lbiza zo veel leuker. Werner, dank dat ik je buiten feestjes en Bergi op kan bellen voor wijze adviezen.

Huize Grevix en Huize Beursman, het zou fantastisch zijn als Chateau Limveau zou slagen. Maar de poging wagen vind ik alleen al briljant. Ik ben dol op onze combinatie van idioten. Dank voor het geïnteresseerd knikken ook al weten jullie niet helemaal waar het over gaat. (Annah, you feel me)

Aernoud, Anne-Meike, Suzanne, Maartje, Plony, Linda en Gea, ik heb jullie tijdens de studie leren kennen maar studievrienden dekt absoluut niet de lading! Dank dat jullie ook altijd het voornemen hebben om het niet over werk te hebben, en daar dan toch in te vervallen. Dokter zijn is nou eenmaal het leukste wat er is!

Plof people, escalatie is ook een vak he! Het wordt weer hoog tijd. Lek luchtbedje in een natte Valk tot een jacht in Kroatië. Zelf gekluste huisfeest, Vastelaovend tot Fusion. Alles is met jullie een avontuur, op de meest relaxte wijze. Ik ben gek op jullie.

Vincent Bloem, die het levensgenieten tot een kunst had verheven. Je wordt enorm gemist.

Familie Baeten \& familie Verheggen, veel dank dat jullie mij zo warm hebben opgenomen in de familie en jullie oprechte interesse in het proefschrift.

Familie van Veenendaal en familie Jansen wil ik ook bedanken voor hun belangstelling. Tante Adrie en familie, in het bijzonder Rianne \& Philip (en natuurlijk Merel en Aimée) jullie betrokkenheid, maaltijdjes en gesprekken zijn heel waardevol voor mij. Enorm bedankt dat ik frequent bij jullie mag aanschuiven. Familie Jansen, met speciale vermelding voor Marcelle en Lex. We zien elkaar minder, wellicht dat het einde van het proefschrift daar verandering in kan brengen, maar jullie verhalen en meeloopdagen in het ziekenhuis hebben zeker bijgedragen aan de keuze voor 
Appendices

Geneeskunde. Daarnaast moeten de ontelbare potten zelfgemaakte sambal ook niet onderschat worden, die hebben mij ongetwijfeld harder hebben doen werken.

Yvo \& Xandra, Marc \& Nadine, (Daan, Lize en Kiki) Mijke \& Peter Jan (Lente) Pieter \& Alessandra, een betere schoonfamilie bestaat er niet. Jullie liefde en betrokkenheid bij elkaar is zo mooi om te zien. Dank dat ik daar onderdeel van mag zijn.

Paranimfen Hendrike Bolkenstein en Josephine Franken. Jullie zijn degene die letterlijk en figuurlijk altijd naast mij staan, niemand anders had mijn paranimf kunnen zijn.

Lieve Dirk, bestaan er woorden die de lading dekken? Ik betwijfel het. Het meeste wordt ook niet gezegd, een blik is genoeg. Neemt niet weg dat slap lullen met een goede fles wijn ook zeker aan ons besteed is. Een betere combinatie bestaat er niet. Je zit in mijn hart.

Lieve Jos, ik moet nog vaak denken aan onze semi-arts stage. We wisten meteen dat het goed zat. Punt. Jij weet als geen ander hoe ik ben, the good and the bad. Van Nick ben ik ook gaan houden. Ik kan en wil niet zonder jullie.

Lieve Rose \& Emiel, Lukas en Senna, kleine zusjes worden groot en dat geldt zeker voor jou, Rose. Je hebt nu jouw eigen prachtige familie en wat doe je dat goed. Toch blijf je ook altijd mijn kleine zusje. Ik houd van je en onze band is me enorm dierbaar. Dank je dat je er bent. Lieve Rens en Miguel, het is soms niet makkelijk om een eigen weg te vinden. Ik houd van je en ben er voor je.

Lieve tante Helga, steun en toeverlaat al heel mijn leven. Je bent er altijd voor me. Ik kan niet uitdrukken hoeveel dat voor mij betekent. Dit boekje is ook voor jou en Harry. Ik houd van je en van onze bijzondere band. 
Lieve Papa en Mama, jullie hebben mij altijd gestimuleerd mijn dromen achterna te gaan. Zonder jullie was ik niet wie ik nu ben en waar ik nu ben. Mama, ik vind het bijzonder dat je de omslag wilde schilderen. Enorm dankbaar ben ik voor de onmetelijke liefde en onvoorwaardelijke steun die jullie altijd aan de kinderen hebben geven, ik houd van jullie.

Allerliefste Michiel, met jou zijn de feestjes leuker, is mijn lach luider, zijn mijn scherpe randjes zachter en hart groter... wat is het leven mooi met jou

- En je kunt niets zeker weten want alles gaat voorbij

Maar ik geloof, ik geloof, ik geloof, ik geloof, ik geloof in jou en mij - 



\section{Curriculum vitae auctoris}

Linde van Veenendaal was born on October 24th 1985 in Nieuwegein, the Netherlands. In 2004 she graduated from secondary school at the Kees Boeke School (Werkplaats Kindergemeenschap) in Bilthoven and started studying Law that same year. In 2008, while obtaining her Bachelor degree in Law, she switched to Medical School at Utrecht University. During her study she was an active member of several student and medical organisations. In the third year of Medical

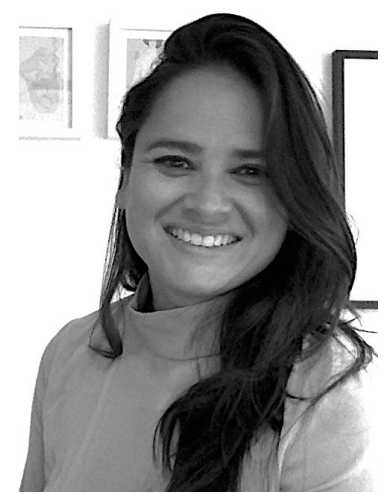
School her interest in surgery resulted in the participation in several surgical research projects. She obtained her medical degree in 2015 after a three-month senior clinical rotation at the department of Surgery of the UMC Utrecht under the supervision of prof. dr. M.R. Vriens. In 2016 she commenced her PhD project under the supervision of prof. dr. G.D. Valk and prof. dr. M.R. Vriens at the Endocrine Cancer Center of the UMC Utrecht and the department of Medical Oncology of the Antoni van Leeuwenhoek - Netherlands Cancer Institute in Amsterdam. Her primary research project concerned the development of a longitudinal prognostic database including patients with neuroendocrine neoplasia treated at their European Neuroendocrine Tumour Society Centre of Excellence. She presented her research at various national and international conferences. In 2019 she started working as a resident not in training at the department of Surgery of the St. Antonius Hospital in Nieuwegein. Currently, Linde lives in Amsterdam with Michiel Baeten and will continue her career as a resident not in training at the department of Surgery of the Radboud UMC in Nijmegen. 



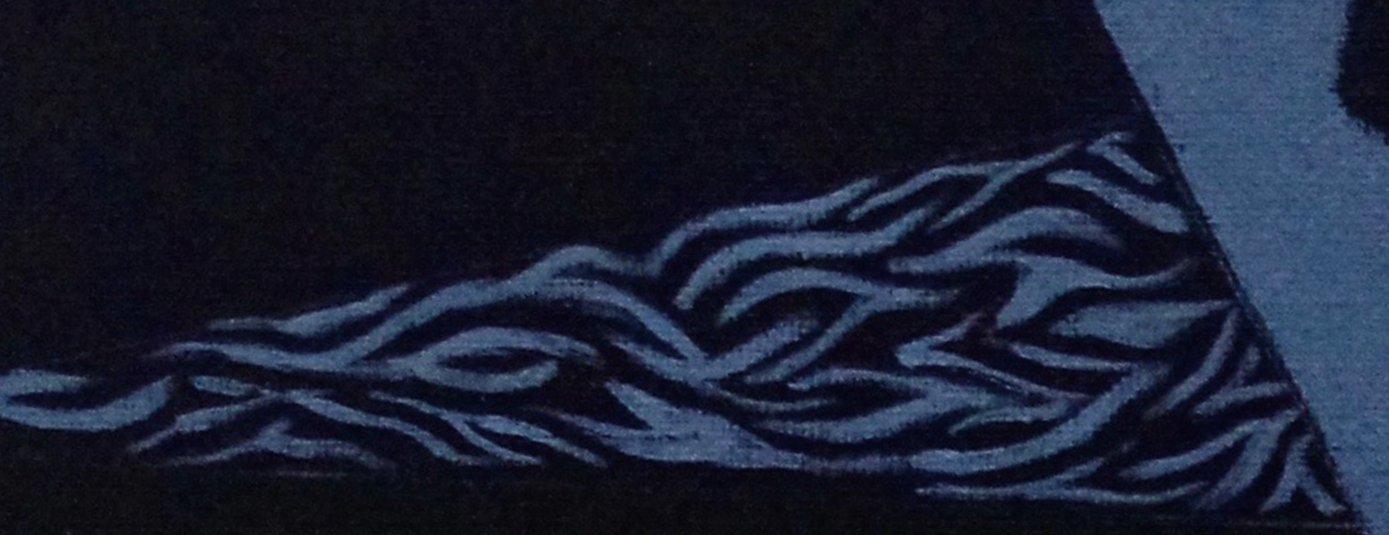

